

\title{
Les équations différentielles algébriques et les singularités mobiles
}

\section{Ivan Pan et Marcos Sebastiani}

Résumé. On considère des équations différentielles ordinaires polynomiales, dites algébriques; parmi celles-ci, on s'intéresse à celles qui n'ont pas de singularité mobile dans le sens classique : on dit que ces équations sont dans la classe de Fuchs-Painlevé. Pour commencer, on fait l'étude locale des équations différentielles algébriques en général et on caractérise de plusieurs façons les équations qui sont dans la classe de Fuchs-Painlevé. À une équation dans cette classe l'on associe un feuilletage analytique génériquement transverse à une fibration sur une surface complexe lisse compacte. Après introduire la notion de genre d'une équation différentielle algébrique, due à Poincaré, on développe la classification birationnelle des équations dans la classe de Fuchs-Painlevé de genre 0 et genre 1 ; dans le cas de genre plus grand que 1 on démontre l'existence d'une integrale première rationnelle. Finalement, on démontre qu'une équation différentielle algébrique qui admet une solution locale ayant une singularité essentielle est forcément dans la classe de Fuchs-Painlevé. 


\section{Table des matières}

$\begin{array}{ll}\text { Introduction } & \text { ix }\end{array}$

Conventions et Notations xiii

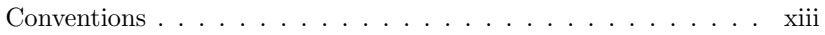

Notations . . . . . . . . . . . . . . . xiv

I L'équation polynomiale $F\left(x, y, y^{\prime}\right)=0 \quad \mathbf{1}$

I.1 Introduction . . . . . . . . . . . . . . 1

I.2 Le théorème généralisé des fonctions implicites . . . . . . 4

I.3 Détermination des solutions . . . . . . . . . . . . . . . . . 9

I.4 La classe de Fuchs-Painlevé . . . . . . . . . . . . . . . . . 14

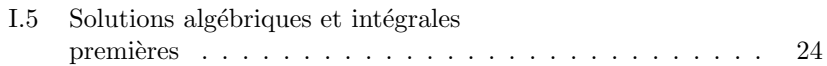

II Équations différentielles algébriques et feuilletages $\quad 31$

II.1 Introduction . . . . . . . . . . . . . . . . . . . . 31

II.2 L'équation polynomiale associée . . . . . . . . . . . . . . 35

II.3 Construction du feuilletage . . . . . . . . . . . . . . . 38

II.4 Solutions méromorphes. . . . . . . . . . . . . . . . . . 50

II.5 Singularités essentielles . . . . . . . . . . . . . . . . . . . . 52

II.6 Le point de vue algébrique . . . . . . . . . . . . . . . . . 54

II.6.1 Dérivations et anneaux de valuation . . . . . . . . 55

II.6.2 Feuilletages et dérivations . . . . . . . . . . . . . . 59

II.6.3 Équations différentielles et dérivations . . . . . . . . 62

III Les équations de genre 0 et de genre $>1$ dans la classe FP 63

III.1 Introduction . . . . . . . . . . . . . . . . . . . . . . 63

III.2 L’image réciproque régulière . . . . . . . . . . . . . . . . 64

III.3 Résultats géométriques . . . . . . . . . . . . . . . . . . 67

III.4 Les équations de genre zéro . . . . . . . . . . . . . . . . 69

III.5 Les équations de Riccati . . . . . . . . . . . . . . . . . . 72

III.6 Les équations de genre $\geq 2 \ldots \ldots \ldots$ 
IV Les équations de genre 1 dans la classe FP 80

IV.1 Introduction . . . . . . . . . . . . . . . . . . . . . . 80

IV.2 Résultats géométriques . . . . . . . . . . . . . . . . . 80

IV.3 Les équations de genre 1 dans la classe FP . . . . . . . . . . 84

IV.4 Séparation de variables . . . . . . . . . . . . . . . . . . . 86

V Équations différentielles algébriques admettant des solu$\begin{array}{ll}\text { tions transcendantes } & 91\end{array}$

V.1 Introduction . . . . . . . . . . . . . . . . . . . . . . . . . 91

V.2 Feuilles transcendantes des feuilletages . . . . . . . . . . . . 92

V.3 Classe FP et solutions transcendantes . . . . . . . . . . . . 99

V.4 Quelques applications ................ . 102

A Feuilletages analytiques et équations de Pfaff sur les surfaces complexes

A.1 Feuilletages réguliers . . . . . . . . . . . . . . . . . . . . . . 112

A.2 Feuilletages à singularités isolées . . . . . . . . . . . . . . . 118

A.3 Les équations de Pfaff . . . . . . . . . . . . . . . . . . . . . 120

A.4 Séparatrices et intégrales premières . . . . . . . . . . . . . 123

A.5 La tangence de deux feuilletages . . . . . . . . . . . . 125

A.6 Feuilletages sur les fibrations . . . . . . . . . . . . . 125

$\begin{array}{lr}\text { Bibliographie } & 129\end{array}$ 


\section{Introduction}

Dans [29] H. Poincaré reprend un travail de M. Fuchs (voir [13]) et étudie les équations différentielles de la forme

$$
F\left(x, y, y^{\prime}\right)=0
$$

avec $F \in \mathbb{C}[x, y, z]$ un polynôme de la forme

$$
\begin{gathered}
F(x, y, z)=c_{0}(x, y) z^{m}+c_{1}(x, y) z^{m-1}+\cdots+c_{m}(x, y), m \geq 1, \\
c_{0}(x, y) \neq 0,
\end{gathered}
$$

tel que l'ensemble des $x=a$ où il existe des solutions locales possédant un point de ramification est fini : dans le langage classique on se réfère à cette situation en disant qu'il n'y a pas de singularité mobile, cette propriété étant connue comme Propriété de Painlevé; nous dirons dans ce cas que (1) est dans la classe de Fuchs-Painlevé. Poincaré définit le genre de (1) et classifie birationnellement ces équations en fonction de celui-ci. En conséquence les solutions de ces équations s'expriment à l'aide de fonctions algébriques, elliptiques et des solutions de l'équation de Riccati.

Par contre, Painlevé a montré que les équations différentielles de second ordre produisent de nouvelles transcendantes.

Le but de cette monographie est d'abord de réécrire [29] dans un langage plus moderne et rigoureux de façon à comprendre les équations dans la classe de Fuchs-Painlevé en termes de feuilletages analytiques sur des surfaces complexes; deuxièmement et à partir de ce nouveau contexte, il s'agit de donner des définitions précises et des démonstrations complètes, de corriger quelques erreurs et de démontrer quelques affirmations dans [29] qui s'y trouvent sans preuve; finalement, et en poussant un peu plus loin les idées de Poincaré, nous obtenons quelques résultats nouveaux : en effet, on constate qu'il est possible de comprendre la situation considérée par Poincaré comme un cas particulier d'une construction plus générale qui donne, en plus, des nouvelles intuitions sur les feuilletages de type Riccati. Pour atteindre cet objectif on s'est servi aussi de [20, chap. XIII] où on expose des résultats de [13] et [29].

Dans cette deuxième édition on a rajouté, d'une part, un paragraphe au chapitre II où on fait le lien entre l'étude des équations différentielles 
algébriques du point de vue purement algébrique, comme l'on trouve dans [27], et notre approche plus géométrique; d'autre part un nouveau chapitre dédié à l'étude, dans le même esprit que notre but initial, des théorèmes de Malmquist sur les équations différentielles avec singularités essentielles (voir [24], [25], [26] [11]).

Afin que notre travail puisse être lu aussi par des éventuels étudiants intéressés par ce sujet, nous avons fait un effort, d'une part pour que notre texte soit essentiellement auto-contenu, et, d'autre part, pour que les techniques utilisées soient les plus élémentaires possible. Plus précisément, on a inclus à la fin du livre, une annexe contenant une petite introduction à l'étude des feuilletages analytiques, qui sert surtout à fixer les notations utilisées tout au long du texte et à évoquer certains des résultats dont on aura besoin dans la suite, ce qui aidera principalement les non-spécialistes; on supposera pourtant que le lecteur est familiarisé avec les notions et résultats fondamentaux de la géométrie algébrique et de plusieurs variables complexes, qui se trouvent d'ailleurs dans la plupart des livres sur ces sujets et pour lesquels on donnera des références précises.

Le texte est divisé en cinq chapitres, numérotés I,II,III,IV et V dont le contenu est détaillé ci-dessous.

Dans le chapitre I on donne d'abord une définition de solution locale qui contient le cas des solutions ramifiées et on utilise le théorème de la séparatrice de Camacho-Sad ([9]) et une généralisation du théorème des fonctions implicites pour établir un théorème d'existence pour l'équation (1); puis on caractérise le fait que l'équation (1) soit dans la classe de Fuchs-Painlevé ou (ce qui généralise cette notion) soit dans la classe de Fuchs-Painlevé faible; ce dernier concept a été défini par Bruno Scárdua.

Finalement, on introduit une notion d'intégrale première rationnelle pour l'équation (1) qu'on caractérise de plusieurs manières.

Comme exemple d'application de la caractérisation de la classe de FuchsPainlevé, on étudie le cas de l'équation dite binomiale, c'est-à-dire, le cas où le polynôme $F(x, y, z)$ est irréductible et de la forme

$$
F(x, y, z)=z^{m}-P(x, y) .
$$

C'est dans le chapitre II qu'on se détache du contexte de Poincaré et on se place dans un autre plus général : on considère des équations de la forme

$$
\left\{\begin{array}{l}
P(x, y, z) d x-Q(x, y, z) d y=0 \\
F(x, y, z)=0
\end{array}\right\}
$$

où $P, Q \in \mathbb{C}[x, y, z]$ sont premiers entre eux et tels que $F$ ne divise pas $Q$. Cela correspond à étudier le feuilletage défini par la forme holomorphe

$$
\omega:=P d x-Q d y
$$

sur la surface d'équation $F(x, y, z)=0$; le cas où $P=z, Q=1$ correspond exactement au cas de l'équation (1). On étend les notions de solution, 
solution ramifiée, intégrale première rationnelle, classe de Fuchs-Painlevé, etc, pour ce type d'équation.

On montre d'abord que l'étude de l'équation ci-dessus correspond, endehors des singularités de la surface $F(x, y, z)=0$, à l'étude d'un feuilletage analytique sur une fibration à fibres connexes $h: X \rightarrow B$ où $X$ est une désingularisation d'une compactification de cette surface et $B$ est une surface de Riemann qui est un revêtement ramifié de la droite projective avec un nombre de feuillets égal au nombre de composantes de la courbe $F(a, y, z)=0$, pour $a$ générique; on définit le genre de l'équation différentielle comme étant le genre de la fibre générique de $h$. Puis on dit que le feuilletage est génériquement transverse à $h$ si les fibres génériques sont transverses au feuilletage et on montre que l'équation est dans la classe de Fuchs-Painlevé si et seulement si le feuilletage associé est génériquement transverse à $h$. Ensuite, on utilise le théorème de Jouanoulou sur l'existence d'intégrale première rationnelle ([21]) pour démontrer un analogue de celui-ci dans le cas de notre équation différentielle, puis un petit paragraphe traite le cas des singularités essentielles : on donne une preuve simple du fait qu'il n'existe pas de singularité essentielle mobile.

Le chapitre s'achève avec un paragraphe dévoué à l'étude des équations différentielles algébriques du point de vue algébrique, comme dans [27]. Ici on caractérise, suivant [11], les équations différentielles algébriques qui sont dans la classe de Fuchs-Painlevé en termes des dérivations du corps des fonctions rationnelles de la surface $X$ introduite plus haut et on montre que cette approche et la nôtre sont équivalentes.

Les chapitres III et IV sont consacrés à la classification birationnelle des équations dans la classe de Fuchs-Painlevé : dans le premier on traite les cas de genre 0 et genre plus grand que 1 et dans le deuxième on considère le cas elliptique. D'après les résultats des chapitres précédents, cela revient à classifier les feuilletages génériquement transverse à une fibration avec fibre générique de genre 0 , plus grand que 1 et 1 , respectivement.

Pour le cas de genre 0 on montre que, quitte à faire un changement de variables, on peut réduire l'équation de départ à une équation de Riccati avec des coefficients des fonctions algébriques de $x$ : l'outil essentiel ici est le théorème de Nöther-Enriques [3, chap.III]. Pour le cas de genre plus grand que 1 , on montre que l'équation différentielle possède une intégrale première rationnelle : dans ce cas, l'utilisation des variétés hyperboliques permet, d'une part de simplifier considérablement la preuve de Poincaré, et d'autre part d'obtenir un résultat un peu plus général. Cela a été repris par Bruno Scárdua dans un contexte plus général ([30]).

Enfin, dans le cas de genre 1 on montre que l'équation différentielle se ramène à une équation à variables séparées qui dépend de la donnée d'une courbe elliptique attachée naturellement à l'équation de départ : ici on démontre un analogue du théorème de Nöther-Enriques pour le cas des fibrations elliptiques de module constant, ce qui nous permet de réutiliser les idées introduites dans le cas de genre 0 . 
Finalement, dans le chapitre $\mathrm{V}$ on étudie les équations différentielles polynomiales qui admettent des solutions ayant une singularité essentielle. On prouve par des méthodes géométriques, que ces équations sont dans la classe de Fuchs-Painlevé (voir [28] et [11]). Ce résultat a été énoncé par J. Malmquist dans [26] dans le cas des solutions entières (globales) et démontré dans des situations spéciales par C. C. Yang et K. Yosida (voir [34], [35]); ce n'est que A. Eremenko qui a repris l'énoncé originel de Malmquist et en a donné une preuve à l'aide de la théorie de Nevanlinna. Notre approche est géométrique et ne demande que l'existence d'une solution locale avec une singularité essentielle.

Plus généralement on démontre, par les mêmes méthodes, que si une solution ayant une singularité essentielle ne rencontre qu'un nombre fini de fois une courbe algébrique, alors cette courbe est aussi une solution.

Si l'équation (1) admet une solution $y=u(x)$ méromorphe transcendante et s'il existe une fonction algébrique telle que l'égalité $u(x)=f(x)$ n'a lieu que pour un nombre fini de valeurs de $x$, alors $y=f(x)$ est aussi solution de (1) et l'équation (1) est birationnellement équivalente à une équation de Riccati. On en déduit que si (1) admet une solution méromorphe transcendante n'ayant qu'un nombre fini de pôles alors les coefficients de (1) vérifient

$$
\operatorname{deg}_{y} c_{k}(x, y) \leq 2 k, 0 \leq k \leq m-1 \text { et } \operatorname{deg}_{y} c_{m}(x, y)<2 m,
$$

ce qui pour $m=1$ implique que (1) est une équation linéaire (théorème de C.C. Yang [34]).

Pour finir on démontre que que si (1) admet une solution transcendante méromorphe n'ayant qu'un nombre fini de pôles et de zéros, alors (1) est une équation linéaire homogène.

Cette monographie fait partie d'un plan de travail qui nous a été proposé par Étienne Ghys à qui nous exprimons ici notre reconnaissance pour ses orientations et encouragements.

Nous remercions aussi le rapporteur pour ses corrections et ses indications qui nous ont permis d'améliorer ce travail. 


\section{Conventions et notations}

\section{Conventions}

Dans ce travail, toutes les variétés algébriques et les espaces analytiques sont considérés sur le corps $\mathbb{C}$ des nombres complexes; on entend par surface un espace analytique de dimension 2 et par surface de Riemann un espace analytique de dimension 1 qui est régulier (ou lisse) et connexe.

On dira pour $a \in \mathbb{C}$ générique, pour signifier pour tout $a \in \mathbb{C}$ sauf un nombre fini. Plus généralement, la notion de générique sera utilisé au sens de la géométrie algébrique.

À l'exception de l'annexe, où on introduit la topologie fine associée à un feuilletage, on utilisera tantôt la topologie transcendante, tantôt la topologie de Zariski ; pour cette dernière on préviendra toujours le lecteur lors de son utilisation tandis que la première sera utilisée sans aucun commentaire. 


\section{Notations}

$F_{z}:$ la dérivée de $F$ par rapport à la variable $z$.

$(X, p)$ : l'espace topologique $X$ pointé en $p$.

$\pi_{1}(X, p)$ : le groupe fondamental de l'espace topologique $X$ centré en $p$.

$\operatorname{deg}_{z} H$ : le degré du polynôme $H$ en la variable $z$.

Sing $W$ : l'ensemble des points singuliers de l'espace analytique $W$.

poids $H$ le poids du germe de polynôme $H$.

$\mathcal{F}(P, Q)$ : le feuilletage associé à la forme différentielle $P d x-Q d y$ sur la surface d'équation $F(x, y, z)=0$.

$\mathbb{C}(W)$ : le corps des fonctions méromorphes sur l'espace analytique irréductible $W$.

$\alpha \wedge \beta$ : le produit extérieur de deux formes différentielles $\alpha$ et $\beta$.

$K / L:$ l'extension de corps $L \subset K$.

$[K: L]:$ le degré de l'extension de corps $K / L$.

$d h(p)$ : différentielle en $p$ de l'application analytique $h$.

$T_{p}(W)$ : l'espace tangent à l'espace analytique $W$ au point régulier $p \in W$.

$\omega(p)$ : valeur, comme application linéaire, de la 1-forme différentielle $\omega$ au point $p$.

ker $T$ : noyau de l'application linéaire $T$.

$D(\mathcal{F}, \mathcal{G})$ : le diviseur de tangence entre les feuilletages $\mathcal{F}$ et $\mathcal{G}$. 


\section{Chapitre I}

\section{L'équation polynomiale $F\left(x, y, y^{\prime}\right)=0$}

\section{I.1 Introduction}

Soit $F \in \mathbb{C}[x, y, z]$ un polynôme irréductible et qui dépend effectivement de $z$. Écrivons :

$$
F(x, y, z)=c_{0}(x, y) z^{m}+c_{1}(x, y) z^{m-1}+\cdots+c_{m}(x, y), m \geq 1, c_{0}(x, y) \neq 0 .
$$

Désignons par $D \in \mathbb{C}[x, y]$ le discriminant de ce polynôme en $z$, c'est-àdire celui obtenu par élimination de $z$ entre $F$ et sa dérivée partielle par rapport à $z$; notons $\Delta \subset \mathbb{C}^{2}$ l'ensemble d'équation $D(x, y)=0$, appelé le lieu discriminant.

Nous allons étudier localement l'équation différentielle

$$
F\left(x, y, y^{\prime}\right)=0
$$

Dans ce chapitre, nous ferons toujours référence à cette équation.

Cette exposé suit, en partie, l'exposé des résultats de Fuchs [13] et Poincaré [29] donné par Ince dans [20], mais nous donnons des énoncés précis et des démonstrations complètes. Ince corrige et complète Poincaré. En particulier, l'affirmation 3-ième de la page 6 de [29] est fausse en l'absence de ramification (voir l'exemple I.4.15 ci-dessous).

La notion de classe de Fuchs-Painlevé faible est due à Bruno Scárdua (voir définition I.4.3 et exemple I.4.16).

Il faut d'abord donner un sens à l'expression : "solution au voisinage de $a \in \mathbb{C}$ ". En première acception, ce serait une fonction analytique $y=y(x)$ au voisinage de $a$ qui satisfait l'équation. Définie de cette manière, cette notion demeure assez restreinte; plus précisément, on voudrait pouvoir dire d'une part que $y=\sqrt{x}$ est une solution de $2 y y^{\prime}-1=0$ au voisinage de 
0 pour contenir le cas des "solutions multiformes", et d'autre part, que $y=1 / x$ est une solution de $y^{\prime}+y^{2}=0$ au voisinage de 0 pour considérer aussi les "solutions passant par l'infini". Il est naturel, alors, d'introduire la notion suivante.

Définition I.1.1. Une solution locale en $a \in \mathbb{C}$ est un couple de fonctions définies au voisinage de $0 \in \mathbb{C}: x=\alpha(t), y=\beta(t)$ où $\alpha(t)$ est analytique et non-constante, et $\beta(t)$ est analytique en dehors de 0 avec au plus un pôle en 0 , telles que:

$$
\alpha(0)=a \text { et } F\left(\alpha(t), \beta(t), \beta^{\prime}(t) / \alpha^{\prime}(t)\right)=0 \text { si } t \neq 0 .
$$

Si $\beta(0)=b \in \mathbb{C} \cup\{\infty\}$, on dit que le couple est une solution par $(a, b)$.

Un changement analytique de paramètre $t=t(s)$ avec $t(0)=0$ et $t(s)$ non-constante, produit une autre solution et on dira que ces deux solutions sont équivalentes. Il est clair que toute solution est équivalente à une solution du type :

$$
x=a+t^{M}, y=\beta(t) .
$$

Si chaque terme non-nul de la série de Laurent de $\beta(t)$ a un exposant multiple de $M$, alors on peut prendre $M=1$ et la solution peut s'écrire sous la forme $y=y(x)$. Dans ce cas on dit que la solution est uniforme. Dans le cas contraire on dira qu'elle est ramifiée.

On notera

$$
\frac{d \beta}{d \alpha}(a):=\lim _{t \rightarrow 0} \frac{\beta^{\prime}(t)}{\alpha^{\prime}(t)} \in \mathbb{C} \cup\{\infty\}
$$

(cette valeur est invariante par équivalence). On appellera cette valeur la pente de la solution. Une solution uniforme de pente finie sera appelée régulière (comme on verra dans le lemme I.3.13 cela équivaut à dire qu'il s'agit d'une solution uniforme par $(a, b)$ avec $b \in \mathbb{C})$.

La raison pour ne pas admettre de singularité essentielle de $\beta(t)$ est que l'on s'intéresse, dans un premier temps, aux solutions analytiques ; c'est-àdire, possédant une relation analytique entre $\alpha(t)$ et $\beta(t)$ au voisinage de $(a, b)$ (voir, pourtant, $\S 5$ du chap. II et chap. V).

En général, on n'a ni existence ni unicité (à équivalence près) des solutions locales par $(a, b)$.

Exemple I.1.2. a) $x y^{\prime}-1=0$. Il n'existe pas de solution locale par $(0,0)$.

b) $y y^{\prime}-x=0$. Il y a deux solutions non-équivalentes par $(0,0)$.

c) $x y^{\prime}-2 y=0$. Toutes les solutions $y=a x^{2}$ passent par $(0,0)$ avec pente 0 . d) $x^{2} y^{\prime}+y=0$. La seule solution locale en 0 est $y=0$, à équivalence près.

Proposition I.1.3. Supposons que $a, b, c \in \mathbb{C}, F(a, b, c)=0, F_{z}(a, b, c) \neq$ 0 . Alors, il existe une solution régulière par $(a, b)$ de pente $c$. De plus, toute solution par $(a, b)$ de pente $c$ est équivalente à celle-là. 
Démonstration. Soit $z=f(x, y)$ analytique au voisinage de $(a, b)$ telle que $f(a, b)=c$, implicite dans $F(x, y, z)=0$.

La première assertion découle du théorème d'existence appliqué à l'équation différentielle explicite $y^{\prime}=f(x, y)$.

Pour la deuxième, supposons que $x=\alpha(t), y=\beta(t)$ définissent une solution par $(a, b)$ de pente $c$ et considérons le système différentiel :

$$
\frac{d x}{d t}=\alpha^{\prime}(t), \frac{d y}{d t}=\alpha^{\prime}(t) f(x, y)
$$

au voisinage de $(0, a, b)$. Alors $x=\alpha(t), y=\beta(t)$ en est une solution avec $\alpha(0)=a$ et $\beta(0)=b$.

D'autre part, il existe $y=g(x)$, analytique au voisinage de $a$, telle que

$$
g^{\prime}(x)=f(x, g(x)) \text { et } g(a)=b \text {. }
$$

Alors, $x=\alpha(t), y=g(\alpha(t))$ est aussi une solution du système qui passe par $(0, a, b)$. Donc, $\beta(t)=g(\alpha(t))$. Alors $x=\alpha(t), y=\beta(t)$ est une solution équivalente à $x=a+t, y=g(a+t)$

Corollaire I.1.4. Si $(a, b) \in \mathbb{C}^{2}, c_{0}(a, b) \neq 0$ et $D(a, b) \neq 0$ alors il existe exactement $m$ solutions par $(a, b)$ (à équivalence près) et elles sont toutes régulières.

Démonstration. Si $x=\alpha(t), y=\beta(t)$ est une solution par $(a, b)$, alors

$$
\frac{d \beta}{d \alpha}(a)
$$

est une racine de l'équation $F(a, b, z)=0$.

Cela résout le problème de l'existence et du nombre de solutions locales par $(a, b)$ quand ce point appartient à l'ouvert de Zariski défini par :

$$
c_{0}(x, y) D(x, y) \neq 0 \text {. }
$$

Nous n'avons pas d'exemple de non-existence de solution locale par $(a, b)$ dans le cas $c_{0}(a, b) \neq 0$ et $D(a, b)=0$. Nous verrons au $\S 3$ du chapitre II que ce problème est en rapport avec le problème de l'existence de séparatrices locales pour un champ de vecteurs dans une surface, au voisinage d'un point singulier de la surface [8].

Pour étudier le problème de l'existence et de la ramification des solutions dans des conditions plus générales, nous avons besoin du théorème généralisé des fonctions implicites, qui revient à construire des paramétrisations locales de la surface $F(x, y, z)=0$ au voisinage d'un point $(a, b, c)$ pour $a$ générique et $(a, b) \in \Delta$. 


\section{I.2 Le théorème généralisé des fonctions im- plicites}

Si $p \in \mathbb{C}^{n}$, l'anneau local de $\mathbb{C}^{n}$ en $p$ est, par définition, l'anneau des germes de fonctions holomorphes au voisinage de $p$.

Pour étudier l'existence de solutions en dehors du lieu discriminant, on s'est servi du théorème des fonctions implicites combiné avec le théorème d'existence et d'unicité des équations différentielles (explicites). Pour l'étude correspondant au voisinage des points de $\Delta$, on va remplacer le premier résultat par sa "version généralisée" et le deuxième par le théorème d'existence de séparatrice locale de Camacho-Sad.

Nous allons considérer le théorème généralisé des fonctions implicites sous la forme suivante :

Théorème I.2.1. Soit $q:=(a, b) \in \Delta$. Supposons que le polynôme $F(a, b, z)$ ne soit pas nul et qu'il existe $c \in \mathbb{C}$ tel que $F(a, b, c)=0$. Supposons aussi qu'au voisinage de $q$, l'ensemble $\Delta$ soit le graphe d'une fonction $y=$ $\eta(x)$ analytique au voisinage de $a(\eta(a)=b)$. Alors, pour chaque facteur irréductible $H$ de $F$ dans l'anneau local de $\mathbb{C}^{3}$ en $p:=(a, b, c)$ il existe un entier $k, m \geq k>0$, et une fonction $z=f(x, v)$ analytique au voisinage de $(a, 0)$ tels que

a) l'équation en $z: H(x, y, z)=0$ possède, au voisinage de c, exactement $k$ racines différentes pour tout $(x, y)$ au voisinage de $q$ avec $y \neq \eta(x)$;

b) $f(a, 0)=c$;

c) $H\left(x, \eta(x)+v^{k}, f(x, v)\right)=0$ au voisinage de $(a, 0)$;

d) si $p(t):=(\alpha(t), \beta(t), \gamma(t)) \in \mathbb{C}^{3}$ est holomorphe, avec $\alpha(t)$ non-constante, au voisinage de $0 \in \mathbb{C}, p(0)=p$ et $H(p(t))=0$, alors il existe $v(t)$ holomorphe au voisinage de $0 \in \mathbb{C}$ telle que

$$
v(0)=0, \beta(t)-\eta(\alpha(t))=v(t)^{k} \text { et } \gamma(t)=f(\alpha(t), v(t)) .
$$

e) Si $\mu$ est une racine primitive $k$-ième de l'unité et $v \neq 0$, alors

$$
f(x, v), f(x, \mu v), \ldots, f\left(x, \mu^{k-1} v\right)
$$

sont tous différents.

Démonstration. Soit $X$ le germe d'ensemble analytique irréductible défini par $H=0$ au voisinage de $p$. Considérons l'application locale :

$$
\pi:(X, p) \rightarrow\left(\mathbb{C}^{2}, q\right), \pi(x, y, z)=(x, y) .
$$

Alors $\pi$ est finie; donc, propre et ouverte. Soit $k$ le degré de $\pi$. Comme $\partial F / \partial z$ ne s'annule pas sur $X-\pi^{-1}(\Delta)$,

$$
\pi: X-\pi^{-1}(\Delta) \longrightarrow U_{q}-\Delta
$$


est un revêtement fini non-ramifié connexe d'ordre $k$, où $U_{q}$ est un voisinage convenable de $q$ que l'on peut supposer de la forme :

$$
U_{q}:=\{(x, y):|x-a|<\epsilon,|y-\eta(x)|<\epsilon\}, \epsilon>0 .
$$

En particulier, le groupe fondamental $\pi_{1}\left(U_{q}-\Delta\right)$ de $U_{q}-\Delta$ est isomorphe à $\mathbb{Z}$. Considérons l'application $\rho: P \rightarrow U_{q}$ définie par

$$
\rho(x, v)=\left(x, \eta(x)+v^{k}\right)
$$

où

$$
P:=\{(x, v):|x-a|<\epsilon,|v|<\sqrt[k]{\epsilon}\}
$$

Alors,

$$
\rho: P-\{v=0\} \longrightarrow U_{q}-\Delta
$$

est un revêtement fini connexe non-ramifié d'ordre $k$. Comme $\pi_{1}\left(U_{q}-\Delta\right)=$ $\mathbb{Z}$, il existe un difféomorphisme analytique :

$$
\varphi: P-\{v=0\} \longrightarrow X-\pi^{-1}(\Delta)
$$

tel que $\rho(u)=\pi(\varphi(u))$ pour tout $u \in P-\{v=0\}$. On voit tout de suite que $\varphi$ se prolonge en une application analytique $\varphi: P \rightarrow \mathbb{C}^{3}$ (par le théorème de Riemann) telle que

$$
\varphi(P) \subset X, \varphi(\{v=0\} \cap P)=\pi^{-1}(\Delta) .
$$

On définit la fonction cherchée $z=f(x, v)$ par :

$$
\varphi(x, v)=\left(x, \eta(x)+v^{k}, f(x, v)\right) .
$$

Les conditions a), b), c) et e) sont immédiatement vérifiées. Pour prouver d) supposons, d'abord, que $\beta(t)=\eta(\alpha(t))$. On prend $v(t)=0$. Si

$$
\gamma(t) \neq f(\alpha(t), v(t))
$$

alors $p(t)$ et $(\alpha(t), \beta(t), f(\alpha(t), 0))$ seraient deux courbes différentes par $p$ contenues dans $\pi^{-1}(\Delta)=\varphi(\{v=0\} \cap P)$, ce qui est absurde.

Supposons maintenant que $\beta(t) \neq \eta(\alpha(t))$. Alors, $p(t) \in X-\pi^{-1}(\Delta)$ si $t \neq 0$. Appliquant $\varphi^{-1}$ on voit qu'il existe $r(t) \in P$ holomorphe au voisinage de $0 \in \mathbb{C}$ telle que $r(0)=(a, 0)$ et $\varphi(r(t))=p(t)$. Soit $r(t)=(\alpha(t), v(t))$. Comme

$$
\varphi(r(t))=\left(\alpha(t), \eta(\alpha(t))+v(t)^{k}, f(\alpha(t), v(t))\right)
$$

on obtient les égalités voulues. 




Figure I.1

Rappelons que si $A$ est un anneau factoriel avec corps des fractions $K$ et $F(z) \in A[z]$ est un polynôme unitaire, tout facteur irréductible unitaire $P(z)$ de $F(z)$ dans $K[z]$ a ses coefficients dans $A$ (voir [23, chap. V, §6]).

Un résultat bien connu (voir [15, vol. II, A 7]) montre que l'anneau local de $\mathbb{C}^{n}$ en un point $p$ est un anneau factoriel.

Proposition I.2.2. Dans les hypothèses et avec les notations du théorème I.2.1, supposons de plus que $c_{0}(a, b) \neq 0$. Soit $A$ l'anneau local de $\mathbb{C}^{2}$ en $q$ et soit $K$ son corps des fractions. Soit $B$ l'anneau local de $\mathbb{C}^{3}$ en p. Alors, les facteurs irréductibles unitaires de $F$ comme éléments de l'anneau $K[z]$ ont leurs coefficients dans $A$. Si $H(x, y, z)$ est un tel facteur et $H(a, b, c)=0$ alors $H$ est un facteur irréductible de $F$ comme élément de $B, c \in \mathbb{C}$ est l'unique racine de $H(a, b, z)=0$ et $k=\operatorname{deg}_{z} H$, degré du polynôme $H$ en la variable $z$.

Démonstration. La première assertion vient du fait que $A$ est un anneau factoriel (voir rappel ci-dessus).

Soit $X$ une composante irréductible du germe d'ensemble analytique défini par $H=0$ au voisinage de $p$. Alors $X=\{G=0\}$ où $G$ est un élément irréductible de $B$ de la forme ([15, vol. II A 4]) :

$$
G(x, y, z)=z^{n}+e_{1}(x, y) z^{n-1}+\cdots+e_{n}(x, y), e_{j} \in A, 1 \leq j \leq n, n>0 .
$$

Effectuons la division de polynômes :

$$
H=G Q+R, Q, R \in A[z] .
$$

Alors, $\left.R\right|_{X}=0$. Comme $\operatorname{deg}_{z} R<n$ et que l'application locale

$$
(X, p) \rightarrow\left(\mathbb{C}^{2}, q\right),(x, y, z) \mapsto(x, y)
$$


est de degré $n$, on a que $R=0$. Donc $H=G, H$ est irréductible dans $B$ et $\operatorname{deg}_{z} H=n$. Nous avons vu au cours de la preuve du théorème I.2.1 que $k=n$. Donc, $\operatorname{deg}_{z} H=k$. La multiplicité de $c$ comme racine de $G(a, b, z)=$ 0 est $n=k$. Donc, $c$ est l'unique racine de $H(a, b, z)=0$.

Exemple I.2.3. a) $x z^{2}-y^{3}=0$ au voisinage de $(a, 0), a \neq 0$ et $c=0$. On a :

$$
\eta(x)=0, k=2, z=(1 / \sqrt{x}) v^{3} .
$$

b) $x^{3} z^{3}-y=0$ au voisinage de $(a, 0), a \neq 0$ et $c=0$. On a :

$$
\eta(x)=0, k=3, z=(1 / x) v .
$$

c) $z^{2}-x\left(y-x^{2}\right)=0$ au voisinage de $(a, a), a \neq 0$ et $c=0$. On a :

$$
\begin{gathered}
z^{2}-x(y-x)^{2}=(z-\sqrt{x}(y-x))(z+\sqrt{x}(y-x)), \\
\eta(x)=x, \text { et } k=1 \text { pour chaque facteur. }
\end{gathered}
$$

Dans le résultat suivant, et plusieurs fois dans la suite de ce travail, nous utiliserons la notion de fonction algébrique ainsi que ses propriétés fondamentales; pour cela nous renvoyons le lecteur à [1, chap. 8].

Proposition I.2.4. Dans les hypothèses et avec les notations du théorème I.2.1 on a, pour $a \in \mathbb{C}$ générique :

$$
f(x, v)-\eta^{\prime}(x)=\sum_{j=r}^{\infty} e_{j}(x) v^{j}, r \geq 0 \text { avec } e_{r}(a) \neq 0 .
$$

Démonstration. Il n'y a rien à démontrer si $D(x, y)$ ne dépend pas de $y$. On supposera donc que $\operatorname{deg}_{y} D(x, y)>0$, ce qui entraîne $\operatorname{deg}_{y} F(x, y, z)>0$.

Comme la dérivée par rapport à $x$ de la fonction algébrique de $x$ définie par $D(x, y)=0$ est aussi une fonction algébrique, il existe $P(x, y) \in \mathbb{C}[x, y]$ tel que $\operatorname{deg}_{y} P>0$ et que si $\theta(x)$ est holomorphe et $D(x, \theta(x))=0$ alors $P\left(x, \theta^{\prime}(x)\right)=0$.

Soit $L$ le sous-ensemble analytique de $\mathbb{C}^{3}$ défini par

$$
L=\{F(x, y, z)=D(x, y)=0\} \cup\{F(x, y, z)=P(x, z)=0\} .
$$

Comme $F$ est irréductible et dépend effectivement de $y$ et de $z$, l'ensemble $L$ est une courbe algébrique. On supposera que $a$ n'est pas dans l'image par la projection $(x, y, z) \mapsto x$ des points singuliers de $L$. Alors, $a$ est générique.

Si $P(a, c) \neq 0$ alors $P(a, f(a, 0)) \neq 0$. Donc $f(a, 0) \neq \eta^{\prime}(a)$ et on a $r=0$ et $e_{0}(a) \neq 0$. Supposons $P(a, c)=0$. Alors $p=(a, b, c)$ est un point nonsingulier de $L$. Par l'absurde supposons que $e_{j}=0$ pour tout $j$ ou que 
$e_{r} \neq 0$ et $e_{r}(a)=0$. Cela implique que le germe d'ensemble analytique au voisinage de $(a, 0) \in \mathbb{C}^{2}$ par

$$
f(x, v)-\eta^{\prime}(x)=0
$$

n'est pas contenu dans $\{v=0\}$. L'image de ce germe par la transformation :

$$
x=x, y=\eta(x)+v^{k}, z=f(x, v)
$$

n'est pas contenue, alors, dans $\{F(x, y, z)=D(x, y)=0\}$ mais elle est contenue dans $L$, puisque $P(x, f(x, v))=P\left(x, \eta^{\prime}(x)\right)=0$. Alors, le germe de $L$ en $p$ n'est pas irréductible : contradiction.

Définition I.2.5. Dans les conditions de I.2.1 et I.2.4, l'entier nonnégatif $r=$ poids $H$ est le poids de $H$ en $p=(a, b, c)$.

Lorsqu'il n'y aura pas de risque de confusion nous omettrons le point $(a, b, c)$ et parlerons tout simplement du poids de $H$.

Lemme I.2.6. Dans les conditions de I.2.1 et I.2.4, pour a $\in \mathbb{C}$ générique, poids $H>0$ équivaut à ce que $y=\eta(x)$ soit une solution par $(a, b)$ de pente c. En plus, dans ce cas on a $H\left(x, \eta(x), \eta^{\prime}(x)\right)=0$.

Démonstration. Si poids $H>0$, on a $f(x, 0)=\eta^{\prime}(x)$. Alors $c=f(a, 0)=$ $\eta^{\prime}(a)$ et $H\left(x, \eta(x), \eta^{\prime}(x)\right)=0$, ce qui implique $F\left(x, \eta(x), \eta^{\prime}(x)\right)=0$.

Supposons que $y=\eta(x)$ soit une solution par $(a, b)$ de pente $c$. Alors

$$
F\left(x, \eta(x), \eta^{\prime}(x)\right)=0 \text { et } c=\eta^{\prime}(a) .
$$

Il suffit de prouver que $H\left(x, \eta(x), \eta^{\prime}(x)\right)=0$. En effet, par I.2.1d on aura $\eta^{\prime}(x)=f(x, 0)$ ce qui entraîne poids $H>0$.

Si $H\left(x, \eta(x), \eta^{\prime}(x)\right) \neq 0$ il existe un facteur irréductible $G$ de $F$ dans l'anneau local de $\mathbb{C}^{3}$ en $p$, non-associé à $H$, tel que $G\left(x, \eta(x), \eta^{\prime}(x)\right)=0$. Soit $z=g(x, v)$ défini pour $G$ comme $z=f(x, v)$ pour $H$ dans le théorème I.2.1. On a $\eta^{\prime}(x)=g(x, 0)$ d'après I.2.1d.

Puisque $a$ est générique, on peut supposer que $p$ est un point non-singulier à tangente non-verticale de la courbe $F=F_{z}=0$. Alors, cette courbe admet une paramétrisation locale au voisinage de $p$ de la forme : $y=$ $\eta(x), z=\theta(x)$.

Comme chaque point de l'ensemble $G=H=0$ est singulier dans la surface $F=0$, cet ensemble est la courbe $y=\eta(x), z=\theta(x)$. Donc,

$$
G(x, \eta(x), \theta(x))=H(x, \eta(x), \theta(x))=0 .
$$

Par I.2.1d on a que $\theta(x)=g(x, 0)=\eta^{\prime}(x)$, ce qui prouve que

$$
H\left(x, \eta(x), \eta^{\prime}(x)\right)=0 .
$$




\section{I.3 Détermination des solutions}

Soient $a, b, c \in \mathbb{C}$ tels que $F(a, b, c)=0$. Supposons que $D(a, b)=0$, que le polynôme $F(a, b, z)$ ne soit pas nul et que $\Delta$ admette une équation locale analytique $y=\eta(x)$ au voisinage de $(a, b)(\eta(b)=a)$. D'après I.2.1, la détermination des solutions locales par $(a, b)$ de pente $c$ se réduit à étudier, pour chaque facteur irréductible $H$ de $F$ dans l'anneau local de $\mathbb{C}^{3}$ en $(a, b, c)$, les solutions par $(a, 0)$ d'une équation du type :

$$
v^{n} \frac{d v}{d x}=g(x, v)
$$

où $g(x, v)$ est analytique au voisinage de $(a, 0)$ (au moyen du changement de la variable dépendante : $\left.y=\eta(x)+v^{k}\right)$. On a, avec les notations de I.2.1 :

$$
n=k-1 \text { et } g=\frac{1}{k}\left(f(x, v)-\eta^{\prime}(x)\right) .
$$

En particulier, nous avons le théorème d'existence suivant, dont la preuve est une application directe du lemme I.3.2 ci-dessous.

Théorème I.3.1. Soit $E(x, y)=0$ une équation minimale de $\Delta$. Soient $a, b \in \mathbb{C}$ tels que $E(a, b)=0, E_{y}(a, b) \neq 0$ et que le polynôme $F(a, b, z)$ ne soit pas nul; prenons $c \in \mathbb{C}$ tel que $F(a, b, c)=0$. Alors, il existe une solution par $(a, b)$ de pente $c$.

Lemme I.3.2. Considérons l'équation

$$
v^{n} \frac{d v}{d x}=g(x, v)
$$

où $n \geq 1$ est un entier et $g$ est holomorphe au voisinage de $(a, 0), a \in \mathbb{C}$. Alors, il existe des fonctions $x=\alpha(t), y=\beta(t)$ holomorphes au voisinage de 0 telles que $\alpha(0)=a, \beta(0)=0, \alpha$ non-constante et :

$$
\beta(t)^{n} \beta^{\prime}(t)=g(\alpha(t), \beta(t)) \alpha^{\prime}(t) .
$$

Démonstration. Supposons $g(x, 0) \neq 0$. Considérons le système autonome :

$$
\frac{d \alpha}{d t}=\beta^{n}, \frac{d \beta}{d t}=g(\alpha, \beta)
$$

au voisinage de $(a, 0)$. Par le théorème de Camacho-Sad [9] il existe une solution locale non-constante (séparatrice) $x=\alpha(t), y=\beta(t)$ de ce système telle que $\alpha(0)=a, \beta(0)=0$; en particulier $\alpha$ n'est pas constante. Si $g(x, 0)=0$ on prend $\alpha=a+t, \beta=0$. 
Exemple I.3.3. Si $F=x z^{2}+\sqrt{2}(x y+y) z+y^{2}$, alors :

$$
D(x, y)=2 y^{2}\left(x^{2}+1\right), E(x, y)=y\left(x^{2}+1\right) .
$$

Donc, $E(0,0)=0, E_{y}(0,0) \neq 0$ mais $F(0,0, z)=0$. Il est facile de voir qu'il n'existe pas de solution par $(0,0)$ de pente 1 pour ce polynôme $F$.

Corollaire I.3.4. Pour $a \in \mathbb{C}$ générique et pour tout $b, c \in \mathbb{C}$ tels que $F(a, b, c)=0$, il existe une solution par $(a, b)$ de pente $c$.

Démonstration. Si $E(a, b) \neq 0$ on applique I.1.3. Si $E(a, b)=0$ on applique I.3.1.

Observation I.3.5. Il est immédiat de voir que si $c_{0}(a, b) \neq 0$ il n'existe pas de solution par $(a, b)$ de pente $\infty$. Pour étudier de telles solutions dans le cas $c_{0}(a, b)=0$ on considère $y$ comme nouvelle variable indépendante et on étudie les solutions par $(a, b)$ de pente 0 et $x$ non-constant de l'équation :

$$
c_{0}(x, y)+c_{1}(x, y) x^{\prime}+\cdots+c_{m}(x, y)\left(x^{\prime}\right)^{m}=0 .
$$

Observation I.3.6. Pour étudier les solutions par $(a, \infty)$ on fait le changement de variable dépendante $y:=1 / y_{1}$ et, en éliminant les dénominateurs, on obtient une équation du type

$$
d_{0}\left(x, y_{1}\right)\left(y_{1}^{\prime}\right)^{m}+d_{1}\left(x, y_{1}\right)\left(y_{1}^{\prime}\right)^{m-1}+\cdots+d_{m}\left(x, y_{1}\right)=0
$$

dont on étudie les solutions par $(a, 0)$ différentes de l'éventuelle solution $y_{1}=0$ (celle-ci correspond à la "solution" $y=\infty$ de (I.1)).

Exemple I.3.7. Considérons l'équation différentielle

$$
\left(y-x^{2}\right)^{2}\left(y^{\prime}\right)^{2}+2\left(y-x^{2}\right)^{2} y^{\prime}+y^{2}=0 .
$$

un calcul direct montre que

$$
D(x, y)=4 x^{2}\left(y-x^{2}\right)^{2}\left(x^{2}-2 y\right), E(x, y)=x\left(y-x^{2}\right)\left(x^{2}-2 y\right) .
$$

Si $a^{2} \neq 2 b, a^{2} \neq b$ et $a \neq 0$ du corollaire I.1.4 suit qu'il existe deux solutions par $(a, b)$. Si $a^{2}=2 b$ et $a \neq 0$ il est de même par la proposition I.1.3. Si $a^{2}=b$ et $a \neq 0$ il existe une solution par $(a, b)$ de pente $\infty$ d'après l'observation I.3.5 et le théorème I.3.1. Par $(0,0)$ nous avons la solution $y=0$. D'après l'observation I.3.6 et le théorème I.3.1, il existe une solution par $(a, \infty)$ si $a \neq 0$.

Théorème I.3.8. Soient $a \in \mathbb{C}$ générique et $b, c \in \mathbb{C}$ tels que $F(a, b, c)=0$ et $D(a, b)=0$. On a les assertions suivantes : 
a) $\Delta$ admet une équation locale analytique $y=\eta(x)$ au voisinage de $(a, b)$;

b) si $y=\eta(x)$ n'est pas solution par $(a, b)$ ou si $\eta^{\prime}(a) \neq c$ alors il existe exactement $n$ solutions par $(a, b)$ de pente $c$ (à équivalence près), où $n$ est le nombre de facteurs irréductibles de $F$ dans l'anneau local de $\mathbb{C}^{3}$ en $(a, b, c)$.

Démonstration. Il est évident que l'assertion a) est vérifié pour a générique, ainsi que la condition $F(a, b, z) \neq 0$. D'après le début de ce paragraphe et I.2.4, pour chaque facteur irréductible $H$ de $F$ dans l'anneau local de $\mathbb{C}^{3}$ en $(a, b, c)$ nous devons considérer les solutions par $(a, 0)$ d'une équation de la forme

$$
k v^{k-1} v^{\prime}=f(x, v)-\eta^{\prime}(x)=\sum_{j=r}^{\infty} e_{j}(x) v^{j},
$$

où les $e_{j}$ sont des fonctions analytiques au voisinage de $a$ et $e_{r}(a) \neq 0$. L'hypothèse de b) implique $r=0$, d'après I.2.6. Si $k=1$ on a existence et unicité.

Soit $k>1$. Il suffit de considérer $v$ comme nouvelle variable indépendante et nous avons, encore, existence et unicité, puisque $v=0$ n'est pas solution de pente $c$, car $r=0$.

Si $n=1$ il n'y a plus rien à démontrer. Si $n>1$, nous devons prouver que les solutions correspondant à des facteurs irréductibles non-associés ne sont pas équivalentes; notons $G$ un tel facteur non-associé à $H$.

Il est clair que $F_{z}$ s'annule sur l'ensemble défini par

$$
H(x, y, z)=G(x, y, z)=0
$$

parce que cet ensemble est constitué de points singuliers de la surface d'équation

$$
F(x, y, z)=0
$$

Donc $y=\eta(x)$ sur cet ensemble.

Supposons que $x=\alpha(t), y=\beta(t)$ définissent une solution par $(a, b)$ de pente $c$ telle que :

$$
H\left(\alpha(t), \beta(t), \frac{\beta^{\prime}(t)}{\alpha^{\prime}(t)}\right)=G\left(\alpha(t), \beta(t), \frac{\beta^{\prime}(t)}{\alpha^{\prime}(t)}\right)=0 .
$$

Alors, $\beta(t)=\eta(\alpha(t))$ d'où suit, par I.2.1d, que $\beta^{\prime}(t) / \alpha^{\prime}(t)=f(\alpha(t), 0)$. Donc, $\eta^{\prime}(\alpha(t))=f(\alpha(t), 0)$, ce qui contredit l'hypothèse de $\left.\mathrm{b}\right)$.

Corollaire I.3.9. Soient $a \in \mathbb{C}$ générique et $b \in \mathbb{C}$ tels que $c_{0}(a, b) \neq 0$ et $D(a, b)=0$. On a les assertions suivantes :

a) $\Delta$ admet une équation locale analytique $y=\eta(x)$ au voisinage de $(a, b)$; 
b) si $y=\eta(x)$ n'est pas solution par $(a, b)$, alors il existe exactement $n$ solutions par $(a, b)$ (à équivalence près), où $n$ est le nombre de facteurs irréductibles de $F$ dans $K[z]$, avec $K$ le corps des fractions de l'anneau local de $\mathbb{C}^{2}$ en $(a, b)$.

Démonstration. Ceci découle de I.3.8, I.3.5 et I.2.2.

Exemple I.3.10. a) $x\left(y^{\prime}\right)^{2}-y^{3}=0$. Si $a \neq 0$, la seule solution par $(a, 0)$ est $y=\eta(x)=0$ (voir I.2.3a)).

b) $x^{3}\left(y^{\prime}\right)^{3}-y=0$. Si $a \neq 0$, il existe deux solutions (à équivalence près) par $(a, 0)$ dont l'une est $y=\eta(x)=0$ (voir I.2.3b)).

c) $\left(y^{\prime}\right)^{2}-x(y-x)^{2}=0$. Si $a \neq 0$, il existe deux solutions (à équivalence près) par $(a, a)$ et $y=\eta(x)$ n'est pas solution (voir I.2.3c)).

d) $\left(y^{\prime}\right)^{2}-2 y^{\prime}+1-x(y-x)^{2}=0$. Si $a \neq 0, D(a, a)=0$ et la seule solution par $(a, a)$ est $y=x$, (à équivalence près), tandis que $F$ est le produit de deux facteurs irréductibles non-associés dans $K[z]$.

Théorème I.3.11. On a les assertions suivantes :

a) Pour $a \in \mathbb{C}$ générique et pour $b, c \in \mathbb{C}$ tels que $F(a, b, c)=0$ il n'existe qu'un nombre fini (à équivalence près) de solutions par $(a, b)$ de pente $c$.

b) Pour $a \in \mathbb{C}$ générique et pour tout $b \in \mathbb{C}$ il existe une solution par $(a, b)$.

Démonstration. a) Si $D(a, b) \neq 0$ il n'existe qu'une solution d'après I.1.3. Si $D(a, b)=0$ nous devons considérer, comme au début de la preuve de I.3.8, une équation du type

$$
k v^{k-1} v^{\prime}=\sum_{j=r}^{\infty} e_{j}(x) v^{j}, e_{r}(a) \neq 0
$$

pour chaque facteur irréductible de $F$ dans l'anneau local de $\mathbb{C}^{3}$ en $(a, b, c)$. Si $k-1 \leq r$, on a une ou deux solutions (dans le deuxième cas $v=0$ est solution). Si $k-1>r$ on considère $v$ comme nouvelle variable indépendante et on a la même chose.

b) Pour $a \in \mathbb{C}$ générique et pour $b \in \mathbb{C}$ tel que $c_{0}(a, b) \neq 0$ il suffit d'appliquer le corollaire I.3.4. Si $c_{0}(a, b)=0$ on peut supposer $c_{0}(a, y) \neq 0$ pour $a$ générique. Alors, par I.3.4 et I.3.5 il existe une solution par $(a, b)$ de pente $\infty$.

Nous finirons ce paragraphe avec deux lemmes qui nous seront utiles plus tard.

Lemme I.3.12. Soit $a \in \mathbb{C}$ tel que $c_{m}(a, y)=0$. Alors, pour $b \in \mathbb{C}$ générique il existe une solution $x=\alpha(t), y=\beta(t)$ par $(a, b)$ de pente 0 telle que $\beta(t)$ n'est pas constante. 
Démonstration. Pour $b \in \mathbb{C}$ générique, $c_{m}(x, b) \neq 0$. Cela implique que si $x=\alpha(t), y=\beta(t)$ est une une solution par $(a, b)$ alors $\beta(t)$ n'est pas constante. Il reste à prouver l'existence d'une solution par $(a, b)$ de pente 0 pour $b \in \mathbb{C}$ générique.

Premier cas : $D(a, y) \neq 0$. Pour $b \in \mathbb{C}$ générique, $D(a, b) \neq 0$. Alors $F(a, b, 0)=0, F_{z}(a, b, 0) \neq 0$ et il suffit d'appliquer I.1.3.

Deuxième cas : $D(a, y)=0$. Pour $b \in \mathbb{C}$ générique le polynôme $F(a, b, z)$ n'est pas nul et $\Delta$ est donné localement au voisinage de $(a, b)$ par l'équation $x=a$. On applique I.2.1 en interchangeant $x$ et $y$. Il en résulte qu'il existe $k \geq 1$ et $z=f(u, y)$, holomorphe au voisinage de $(0, b)$, tels que :

$$
f(0, b)=0 \text {, et } F\left(a+u^{k}, y, f(u, y)\right)=0 \text { au voisinage de }(0, b) .
$$

La solution $y=g(u)$ telle que $g(0)=b$, de l'équation

$$
\frac{d y}{d u}=\frac{d y}{d x} \frac{d x}{d u}=k f(u, y) u^{k-1}
$$

nous donne la solution $x=a+u^{k}, y=g(u)$ de $F\left(x, y, y^{\prime}\right)=0$, qui est une solution par $(a, b)$ de pente 0 .

Lemme I.3.13. Toute solution par $(a, b)$, où $b \in \mathbb{C}$, de pente $\infty$, est ramifiée.

Démonstration. Si

$$
x=a+t^{M}, y=b+\sum_{k=r}^{\infty} b_{k} t^{k}, 1 \leq r<M, b_{r} \neq 0
$$

est une solution par $(a, b)$ de pente $\infty$, alors $M$ ne divise pas $r$.

Exemple I.3.14. Considérons l'équation

$$
\left(y^{\prime}\right)^{m}+A(x, y)=0,
$$

appelée l'équation binomiale. Nous supposerons

$$
A(x, y)=P_{1}(x, y) \cdots P_{n}(x, y)
$$

où les $P_{j}$ sont des polynômes irréductibles non-associés deux à deux et où aucun d'entre eux n'est indépendant de $x$. Alors le polynôme $F(x, y, z)=$ $z^{m}+A(x, y)$ est irréductible, non seulement dans $\mathbb{C}[x, y, z]$, mais aussi dans $K[z]$, où $K$ est le corps de fractions de l'anneau local de $\mathbb{C}^{2}$ en n'importe quel point de $\Delta$ : en effet, il suffit d'appliquer le critère d'Einsenstein, puisque $D(x, y)=A(x, y)$. 
Observation I.3.15. Supposons que $y=\eta(x)$ soit une solution telle que $D(x, \eta(x))=0$. Alors, $\eta^{\prime}(x)=0$. Donc $\eta(x)=b$ constante et $A(x, b)=0$ : contradiction.

De ce qui précède et de I.1.3 I.2.2 et I.3.8 on déduit que pour $a \in \mathbb{C}$ générique et pour $b, c \in \mathbb{C}$ tels que $F(a, b, c)=0$ il existe une et une seule solution, à équivalence près, passant par $(a, b)$ de pente $c$.

Supposons que $F \in \mathbb{R}[x, y, z]$ et $a, b \in \mathbb{R}$. Soit

$$
x=a+t^{M}, y=\beta(t)=b+\sum_{j=1}^{\infty} b_{j} t^{j}
$$

une solution par $(a, b)$ de pente $c \in \mathbb{R}$. Alors

$$
x=a+t^{M}, y=b+\sum_{j=1}^{\infty} \overline{b_{j}} t^{j}=\overline{\beta(\bar{t})}
$$

est aussi une solution par $(a, b)$ de pente $c$. Donc, elle est équivalente à la première, d'après ce qui précède. On voit tout de suite que cela implique qu'il existe $\epsilon \in \mathbb{C}$ tel que :

$$
\epsilon^{M}=1 \text { et } b_{j} \epsilon^{j}=\overline{b_{j}}, j=1,2, \ldots
$$

Soit $\epsilon=\eta^{2}$. Alors $\eta^{M}= \pm 1$. En plus, $b_{j} \eta^{j} \in \mathbb{R}$ pour $j=1,2, \ldots$ En faisant $t=\eta s$ dans la première solution on obtient la solution analytique réelle :

$$
x=a \pm s^{M}, y=b+\sum_{j=1}^{\infty}\left(b_{j} \eta^{j}\right) s^{j} .
$$

Par exemple, par chaque point $(a, a) \in \mathbb{R}^{2}$ passe une solution analytique réelle de $\left(y^{\prime}\right)^{2}+x-y=0$ qui a un point de rebroussement à tangente horizontale en $(a, a)$ (faire $\left.y=x+v^{2}\right)$.

\section{I.4 La classe de Fuchs-Painlevé}

Dans certaines équations les solutions locales ramifiées n'apparaissent qu'en certains points fixes; par exemple, les solutions locales en $a \in \mathbb{C}$ de l'équation

$$
4 x\left(y^{\prime}\right)^{2}-1=0
$$

ne sont pas ramifiées à moins que $a=0$. Par contre, dans d'autres cas, le point où se présente la ramification dépend de la solution (dans la littérature classique on se réfère à cette situation en disant qu'on a des "singularités mobiles"); par exemple, pour tout $a \in \mathbb{C}$ l'équation

$$
2 y y^{\prime}-1=0
$$


possède la solution ramifiée par $(a, 0)$ définie par :

$$
x=a+t^{2}, y=t .
$$

Définition I.4.1. On dit que $a \in \mathbb{C}$ est un point de ramification de l'équation s'il existe une solution locale en a qui soit ramifiée.

Définition I.4.2. On dit que l'équation (I.1) est dans la classe de FuchsPainlevé, ce que l'on note FP s'il n'existe qu'un nombre fini de points de ramification.

C'est-à-dire, l'équation est dans la classe $\mathrm{FP}$ si pour $a \in \mathbb{C}$ générique, toute solution locale en $a$ est uniforme (même les solution par $(a, \infty)$ s'il en existe).

D'après I.1.4 on sait que si $a, b \in \mathbb{C}$ et $c_{0}(a, b) D(a, b) \neq 0$, alors toute solution par $(a, b)$ est uniforme.

Définition I.4.3. On dit que l'équation (I.1) est dans la classe de Fuchs-

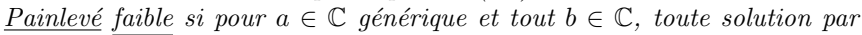
$(a, b)$ est uniforme.

Cela veut dire que les éventuelles ramifications "mobiles" ne se présentent pas à distance finie.

Exemple I.4.4. a) $x\left(y^{\prime}\right)^{2}-y^{3}=0$. D'après I.2.3a et I.3.6, pour $a \in \mathbb{C}$, $a \neq 0$, la seule solution par $(a, 0)$ est $y=\eta(x)=0$ et il n'y a pas de solution ramifiée par $(a, \infty)$. Cette équation est dans la classe FP.

b) $x^{3}\left(y^{\prime}\right)^{3}-y=0$. D'après I.2.3b, cette équation n'est pas dans la classe FP faible puisqu'elle a la solution ramifiée par $(a, 0), a \neq 0$, suivante :

$$
x=a e^{\frac{3}{2} \mathrm{t}^{2}}, y=t^{3} .
$$

On vérifie qu'il n'existe pas de solution locale en $a=0$. Donc, pour cette équation, l'ensemble des points de ramification est $\mathbb{C}-\{0\}$.

c) $2 y^{\prime}+y^{3}=0$ est dans la classe FP faible mais elle n'est pas dans la classe FP.

Dans ce qui suit on va chercher des conditions nécessaires et suffisantes pour que l'équation (I.1) soit dans la classe FP faible et dans la classe FP respectivement.

Lemme I.4.5. Pour $a \in \mathbb{C}$ générique et pour $b \in \mathbb{C}$ tels que $c_{0}(a, b) \neq 0$ et $D(a, b)=0$, on $a$ a) $\Rightarrow b$ ) et $b) \Rightarrow c)$ :

a) toute solution par $\left(a^{\prime}, b^{\prime}\right)$ est uniforme pour $a^{\prime}$ au voisinage de a et $b^{\prime}=\eta\left(a^{\prime}\right)$.

b) poids $H \geq \operatorname{deg}_{z} H-1$ pour chaque facteur irréductible unitaire $H$ de $F$ dans $K[z]$ où $K$ est le corps de fractions de l'anneau local de $\mathbb{C}^{2}$ en $(a, b)$ (poids $H$ est bien défini : voir I.2.2 et I.2.5);

c) toute solution par $(a, b)$ est uniforme. 
Démonstration. 1. $a) \Rightarrow b$ ). Soit $D_{a}$ un petit disque de centre $a$. Nous pouvons supposer que toute solution locale par $\left(a^{\prime}, b^{\prime}\right)$ est uniforme si $a^{\prime} \in$ $D_{a}$ et $b^{\prime}=\eta\left(a^{\prime}\right)$ et que $\Delta$ est défini localement, au voisinage de $(a, b)$, par une équation $y=\eta(x)$ analytique dans $D_{a}$ avec $b=\eta(a)$. En reprenant le début du paragraphe $\S 3$, pour chaque $H$ on a une équation

$$
k v^{k-1} v^{\prime}=f(x, v)-\eta^{\prime}(x)=\sum_{j=r}^{\infty} e_{j}(x) v^{j}
$$

où les $e_{j}$ sont analytiques dans $D_{a}$, avec $e_{r}\left(a^{\prime}\right) \neq 0$ pour tout $a^{\prime} \in D_{a}$ (voir I.2.4) où $r:=$ poids $H$ et $k=\operatorname{deg}_{z} H$ (voir I.2.1 et I.2.2).

On peut supposer que $c_{0}\left(a^{\prime}, b^{\prime}\right) \neq 0$ pour tout $a^{\prime} \in D_{a}$ où $b^{\prime}=\eta\left(a^{\prime}\right)$. Supposons, par l'absurde, que $k>r+1$.

En prenant $v$ comme nouvelle variable indépendante on est amené à une équation de la forme

$$
\frac{d x}{d v}=v^{n} h(x, v), 0<n=k-1-r \leq k-1
$$

où $h$ est holomorphe et jamais nulle dans $D_{a} \times D, D$ étant un disque de centre 0 dans $\mathbb{C}$. Soit $a^{\prime} \in D_{a}$ et soit $x=\alpha(v)$ la solution de cette équation au voisinage de 0 telle que $\alpha(0)=a^{\prime}$. Alors,

$$
x=\alpha(v), y=\eta(\alpha(v))+v^{k}
$$

est une solution locale par $\left(a^{\prime}, b^{\prime}\right)$ de l'équation $F\left(x, y, y^{\prime}\right)=0$ où $b^{\prime}=\eta\left(a^{\prime}\right)$. Donc elle est uniforme (et de pente finie), ce qui veut dire qu'il existe $y=\varphi(x)$ holomorphe au voisinage de $a^{\prime}$ telle que

$$
\varphi\left(a^{\prime}\right)=b^{\prime} \text { et } \eta(\alpha(v))+v^{k}=\varphi(\alpha(v)) .
$$

Alors :

$$
(\varphi-\eta)(\alpha(v))=v^{k}
$$

D'après le lemme I.4.6 ci-dessous il existe un diviseur $l>1$ de $k$ tel que

$$
\alpha(\epsilon v)=\alpha(v) \text { où } \epsilon=\exp (2 \pi i / l) .
$$

Comme $\alpha(v)-a^{\prime}$ s'annule en 0 avec multiplicité $n+1$, l'entier $l$ divise $n+1$ (donc, il divise $r$ ).

D'après le lemme I.4.7 ci-dessous, $h(x, \epsilon v)=h(x, v)$. On en déduit que $f(x, \epsilon v)=f(x, v)$ puisque $l$ divise $r$. Cela contredit I.2.1e.

2. b) $\Rightarrow c$ ). On commence comme dans 1 . et on observe que quand $r \geq k-1$ l'équation

$$
k v^{k-1} \frac{d v}{d x}=\sum_{j=r}^{\infty} e_{j}(x) v^{j}
$$


n'admet que la solution $v=0$ (éventuellement) et une autre $v=v(x)$ analytique. Comme $c_{0}(a, b) \neq 0$, il n'y a pas de solution de pente $\infty$ par $(a, b)$. Cela montre que toute solution par $(a, b)$ est uniforme.

Lemme I.4.6. Soit $u$ une fonction holomorphe au voisinage de $0 \in \mathbb{C}$ telle que $u^{\prime}(0)=0$. Soit $a:=u(0)$ et supposons qu'il existe une fonction $w$ holomorphe au voisinage de a telle que $w(u(z))=z^{k}$ où $k>1$. Alors, il existe un diviseur $l>1$ de $k$ tel que $u(\epsilon z)=u(z)$ pour tout $\epsilon$ racine l-ième de l'unité.

Démonstration. Si $z, z^{\prime}$, au voisinage de 0 , sont tels que $u(z)=u\left(z^{\prime}\right)$, alors il existe $\theta$, racine $k$-ième de l'unité, telle que $z^{\prime}=\theta z$. Soit $z_{n} \rightarrow 0, z_{n} \neq 0$. Il existe $z_{n}^{\prime} \neq z_{n}, z_{n}^{\prime} \neq 0, z_{n}^{\prime} \rightarrow 0$ avec $u\left(z_{n}^{\prime}\right)=u\left(z_{n}\right)$, car $u^{\prime}(0)=0$. Alors, $z_{n}^{\prime}=\theta_{n} z_{n}$ où $\left(\theta_{n}\right)^{k}=1$ et $\theta_{n} \neq 1$ pour tout $n$. Puisqu'il n'y a qu'un nombre fini de racines $k$-ièmes de l'unité, on peut supposer $\theta_{n}=\epsilon$ constante. Alors $u(z)-u(\epsilon z)=0$ et $\epsilon$ est une racine $l$-ième primitive de l'unité pour $l>1$ diviseur de $k$.

Lemme I.4.7. Soit $a \in \mathbb{C}$ et considérons l'équation différentielle

$$
\frac{d w}{d z}=z^{l-1} g(z, w), l>1
$$

où $g$ est holomorphe au voisinage de $(0, a)$. Soit $w=w(z, u)$, holomorphe au voisinage de $(0, a)$, la solution telle que $w(0, u)=u$. Supposons qu'il existe $\epsilon$ racine l-ième de l'unité telle que $w(\epsilon z, u)=w(z, u)$. Alors, $g(\epsilon z, w)=$ $g(z, w)$.

Démonstration. En dérivant nous avons

$$
\frac{\partial w}{\partial z}(\epsilon z, u) \epsilon=\frac{\partial w}{\partial z}(z, u)
$$

ce qui entraîne

$$
g(\epsilon z, w(z, u))=g(z, w(z, u)) .
$$

Pour finir la preuve on observe que l'application locale

$$
\left(\mathbb{C}^{2},(0, a)\right) \rightarrow\left(\mathbb{C}^{2},(0, a)\right),(z, u) \mapsto(z, w(z, u))
$$

est surjective : en effet, puisque $w(0, u)=u$, nous avons $w_{u}(0, a)=1$ et donc le jacobien de l'application ci-dessus est non-nul en $(0, a)$.

Observation I.4.8. Si poids $H<\operatorname{deg}_{z} H-1$, le lemme I.4.5 implique l'existence d'une suite (non-constante) de points de ramification de l'équation (I.1), qui tend vers $a$. 
Proposition I.4.9. Si l'équation I.1 est dans la classe FP faible, alors $c_{0}(x, y)$ ne dépend pas de $y$.

Démonstration. Supposons que $c_{0}(x, y)$ dépend effectivement de $y$. Nous allons démontrer que pour un $a \in \mathbb{C}$ générique et pour un $b \in \mathbb{C}$ convenable, il existe une solution par $(a, b)$ de pente $\infty$. Il suffira, alors, d'appliquer I.3.13.

Soit $L \subset \mathbb{C}^{2}$ la courbe définie par $c_{0}(x, y)=0$. Alors $L$ contient une composante irréductible $M$ qui n'est pas une droite verticale.

Cas 1 : $M$ n'est pas une droite horizontale. En procédant comme dans l'observation I.3.5 on considère l'équation :

$$
c_{0}(x, y)+c_{1}(x, y) x^{\prime}+\cdots+c_{m}(x, y)\left(x^{\prime}\right)^{m}=0 .
$$

Pour $b \in \mathbb{C}$ générique on peut supposer que :

a) $b$ appartient à l'image de $M$ par la projection $(x, y) \mapsto y$ mais pas à l'image de $\operatorname{Sing} L$, et

b) si $(a, b) \in M$, il existe une solution par $(a, b)$ de pente 0 de cette équation (voir corollaire I.3.4). Si cette solution était $x=a$ on aurait $c_{0}(a, y)=0$. Mais alors $\{x=a\} \subset L$ et $(a, b)$ serait un point singulier de $L$; contradiction. Donc cette solution nous donne une solution de $F\left(x, y, y^{\prime}\right)=$ 0 par $(a, b)$ de pente $\infty$. L'affirmation ci-dessus en résulte tout de suite. Cas 2: $M$ est une droite horizontale. On fait de même que dans le premier cas et on applique I.3.12.

Proposition I.4.10. Si l'équation I.1 est dans la classe FP, alors

$$
\operatorname{deg}_{y} c_{k}(x, y) \leq 2 k \quad(k=0,1, \ldots, m) .
$$

Démonstration. Nous avons déjà prouvé dans I.4.9 que $c_{0}(x, y)=c_{0}(x)$. Posons :

$$
s_{k}:=\operatorname{deg}_{y} c_{k}(x, y) \text { pour } k=0,1, \ldots, m .
$$

En faisant $y=1 / y_{1}$ on obtient l'équation

$$
c_{0}(x)\left(-\frac{y_{1}^{\prime}}{y_{1}^{2}}\right)^{m}+c_{1}\left(x, \frac{1}{y_{1}}\right)\left(-\frac{y_{1}^{\prime}}{y_{1}^{2}}\right)^{m-1}+\cdots+c_{m}\left(x, \frac{1}{y_{1}}\right)=0 .
$$

Soit $N:=\sup _{k}\left(s_{k}+2 m-2 k\right)$. En multipliant cette équation par $y_{1}^{N}$ on obtient une équation du type $F_{1}\left(x, y_{1}, y_{1}^{\prime}\right)=0$ où

$$
F_{1}\left(x, y_{1}, z_{1}\right)=d_{0}\left(x, y_{1}\right) z_{1}^{m}+d_{1}\left(x, y_{1}\right) z_{1}^{m-1}+\cdots+d_{m}\left(x, y_{1}\right)
$$

est un polynôme irréductible. Toute solution ramifiée par $(a, 0)$ de $F_{1}\left(x, y_{1}, y_{1}^{\prime}\right)=0$ donne lieu à une solution ramifiée par $(a, \infty)$ de $F\left(x, y, y^{\prime}\right)=0$. 
Si $s_{k}>2 k$ pour quelque $k$ alors $N>2 m$, ce qui entraîne $d_{0}(x, 0)=0$. Par I.3.5 et I.3.12 on sait qu'il existe, pour $a \in \mathbb{C}$ générique, une solution par $(a, 0)$ de pente $\infty$ de $F_{1}\left(x, y_{1}, y_{1}^{\prime}\right)=0$. D'après I.3.13, cette solution est ramifiée. Donc, l'équation $F\left(x, y, y^{\prime}\right)=0$ n'est pas dans la classe FP.

Les quatre résultats qui suivent résument les renseignements relatifs à l'équation (I.1) obtenus jusqu'à maintenant.

Théorème I.4.11. L'équation (I.1) est dans la classe FP faible si et seulement si les deux conditions suivantes sont satisfaites :

A) $c_{0}(x, y)$ ne dépend que de $x$;

B) pour $a \in \mathbb{C}$ générique et $b \in \mathbb{C}$ tel que $D(a, b)=0$ on $a$ :

$$
\text { poids } H \geq \operatorname{deg}_{z} H-1
$$

pour chaque facteur irréductible unitaire $H$ de $F$ dans $K[z]$, où $K$ est le corps de fractions de l'anneau local de $\mathbb{C}^{2}$ en $(a, b)$.

Démonstration. Si l'équation est dans la classe FP faible, alors A) résulte de I.4.9 et B) de I.4.5.

Supposons vérifiées A) et B). Pour $a \in \mathbb{C}$ générique, $c_{0}(a) \neq 0$.

Si $b \in \mathbb{C}$ et $D(a, b) \neq 0$ toute solution par $(a, b)$ est uniforme par I.1.4.

Si $b \in \mathbb{C}$ et $D(a, b)=0$ on a la même conclusion d'après I.4.5

Proposition I.4.12. Supposons que l'équation (I.1) soit dans la classe FP faible. Pour $a \in \mathbb{C}$ générique et $b \in \mathbb{C}$ tel que $D(a, b)=0$, soit $y=\eta(x)$ une équation locale de $\Delta$ au voisinage de $(a, b)(\eta(a)=b)$. Supposons que $F$ admette un facteur irréductible unitaire $H$ dans l'anneau $K[z]$, où $K$ est le corps de fractions de l'anneau local de $\mathbb{C}^{2}$ en $(a, b)$, avec $\operatorname{deg}_{z} H>1$. Alors $y=\eta(x)$ est une solution régulière et $H\left(x, \eta(x), \eta^{\prime}(x)\right)=0$.

Démonstration. D'après I.4.9 $c_{0}(x, y)=c_{0}(x)$ et on peut supposer $c_{0}(a) \neq$ 0. Par I.2.2 et I.4.5 on conclut que poids $H>0$. La proposition suit de I.2.6.

Théorème I.4.13. Supposons que l'équation (I.1) soit dans la classe FP faible. Alors elle est dans la classe FP si et seulement si les deux conditions suivantes sont satisfaites :

$\left.A^{\prime}\right) \operatorname{deg}_{y} c_{k}(x, y) \leq 2 k, k=1,2, \ldots, m$;

B') si le discriminant, comme polynôme en $z_{1}$, de

$$
\begin{aligned}
F_{1}\left(x, y_{1}, z_{1}\right)= & (-1)^{m} c_{0}(x) z_{1}^{m}+(-1)^{m-1} y_{1}^{2} c_{1}\left(x, \frac{1}{y_{1}}\right) z_{1}^{m-1} \\
& +\cdots+y_{1}^{2 m} c_{m}\left(x, \frac{1}{y_{1}}\right)
\end{aligned}
$$


(qui est un polynôme irréductible) contient le facteur $y_{1}$, alors, pour $a \in \mathbb{C}$ générique

$$
\text { poids } H \geq \operatorname{deg}_{z} H-1
$$

pour chaque facteur irréductible unitaire $H$ de $F_{1}$ dans $K\left[z_{1}\right]$, où $K$ est le corps de fractions de l'anneau local de $\mathbb{C}^{2}$ en $(a, 0)$.

Démonstration. De manière analogue à ce qu'on a fait lors de la preuve de I.4.11, l'assertion suit de I.1.4, I.4.10, I.3.5, I.3.6 et I.4.5.

Gardons les notations de I.4.13

Proposition I.4.14. Supposons que l'équation I.1 soit dans la classe FP. Si le discriminant de $F_{1}$ contient le facteur $y_{1}$ et s'il existe un facteur irréductible $H$ de $F_{1}$ dans $K\left[z_{1}\right]$ dont le degré en $z_{1}$ est $>0$, alors

$$
\operatorname{deg}_{y} c_{m-1}(x, y)<2 m-2 \text { et } \operatorname{deg}_{y} c_{m}(x, y)<2 m \text {. }
$$

Démonstration. Par le même raisonnement que celui de la preuve de I.4.12 appliqué à l'équation $F_{1}\left(x, y_{1}, y_{1}^{\prime}\right)=0$, on a que $H(x, 0,0)=0$. Alors, d'après I.2.2, $z_{1}=0$ est une racine multiple de $F_{1}\left(a, 0, z_{1}\right)=0$. Comme ceci vaut pour $a$ générique, le coefficient de $z_{1}$ et le terme indépendant dans $F_{1}$ s'annulent lorsque $y_{1}=0$. La proposition en résulte aussitôt.

Exemple I.4.15. $\left(y^{\prime}\right)^{2}-y(x-y)^{2}=0$. Nous allons vérifier que cette équation est dans la classe FP. On a

$$
D(x, y)=4 y(x-y)^{2}, \Delta=\{y=0\} \cup\{y=x\} .
$$

On distingue trois cas :

1) $a \neq 0$ et $b \neq 0, a$. Toute solution par $(a, b)$ est uniforme d'après I.1.4 et I.3.5.

2) $a \neq 0$ et $b=0$. Le polynôme

$$
F(x, y, z)=z^{2}-y(y-x)^{2}
$$

est irréductible dans $K[z]$, où $K$ est le corps de fractions de l'anneau local de $\mathbb{C}^{2}$ en $(a, b)$. On fait $y=v^{2}$ et on obtient

$$
\text { poids } F=1 \text { et } \operatorname{deg}_{z} F=2 \text {. }
$$

3) $a \neq 0$ et $b=a$. Le polynôme $F$ se factorise alors en deux facteurs linéaires dans $K[z]$

D'après I.4.11 l'équation est déjà dans la classe FP faible. 
Considérons maintenant :

$$
F_{1}\left(x, y_{1}, z_{1}\right)=y_{1}^{4}\left(\left(-\frac{z_{1}}{y_{1}^{2}}\right)^{2}-\frac{1}{y_{1}}\left(x-\frac{1}{y_{1}}\right)^{2}\right)=z_{1}^{2}-y_{1}\left(x y_{1}-1\right)^{2} .
$$

Alors $F_{1}$ est irréductible dans $K[z]$ pour $a \neq 0$ et $b=0$. En posant $y_{1}=$ $\left(v_{1}\right)^{2}$ on voit que

$$
\text { poids } F=1 \text { et } \operatorname{deg}_{z} F=2 \text {. }
$$

Donc, l'équation est dans la classe FP par I.4.13.

Observons que $y=x$ est implicite dans

$$
F(x, y, z)=F_{z}(x, y, z)=0
$$

et pourtant $y=x$ n'est pas solution. Cela montre que l'affirmation numéro 3 de la page 6 de [29] est fausse ( $F$ est irréductible, comme polynôme en $y, z$ sur la clôture algébrique de $\mathbb{C}(x))$.

Exemple I.4.16. Si $m=1$ alors l'équation est dans la classe FP faible si et seulement si $c_{0}(x, y)$ ne dépend pas de $y$ et elle est dans la classe FP si et seulement $\operatorname{si~} \operatorname{deg}_{y} c_{j}(x, y) \leq 2 j$ pour $j=0,1$. Dans ce dernier cas on a une équation de Riccati.

Exemple I.4.17. On considère l'équation binomiale

$$
\left(y^{\prime}\right)^{m}-P(x, y)=0
$$

où $P \in \mathbb{C}[x, y]$; nous en avons déjà considéré un cas particulier dans I.3.14 (voir aussi $[20, \S 13.8]$ ). On suppose que

$$
F(x, y, z)=z^{m}-P(x, y)
$$

est irréductible et que l'équation (I.1) est dans la classe FP. Si $m=1$, l'équation est de Riccati d'après I.4.16. On supposera $m>1$. Par I.4.13, $\operatorname{deg}_{y} P \leq 2 m$. Supposons pour un instant que $\operatorname{deg}_{y} P<2 m$; soit $c \in \mathbb{C}$ tel que $P(x, c) \neq 0$.

Le changement de variable

$$
y:=\frac{1}{\eta}+c
$$

ramène l'équation au cas où $\operatorname{deg}_{y} P=2 m$, ce que l'on supposera désormais.

En décomposant $P$ en facteurs premiers on obtient

$$
P(x, y)=B(x) B_{1}(x, y)^{k_{1}} \cdots B_{r}(x, y)^{k_{r}}, k_{1} \geq \cdots \geq k_{r}>0,
$$

avec $B \in \mathbb{C}[x], B_{j} \in \mathbb{C}[x, y], j=1, \ldots, r$, où les $B_{j}$ premiers et deux à deux non-associés; de plus on doit avoir

$$
\sum_{j=1}^{r} k_{j} \operatorname{deg}_{y} B_{j}=2 m \text { et } \operatorname{deg}_{y} B_{j}>0,1 \leq j \leq r .
$$


En particulier, $k_{1} \leq 2 m$.

Supposons d'abord que, pour un $j$ particulier, $k_{j} \neq m, 2 m$. Notons

$$
d_{j}:=\operatorname{pgcd}\left(m, k_{j}\right), a_{j}:=m / d_{j}, l_{j}:=k_{j} / d_{j} .
$$

Soit $(a, b) \in \mathbb{C}^{2}$ avec $B_{j}(a, b)=0$. Pour $a$ générique, soit $y=\eta(x)$ l'équation locale de l'ensemble des zéros de $B_{j}$ au voisinage de $(a, b)$. Le polynôme $F$ admet un facteur sur le corps des fractions $K$ de l'anneau local $A$ de $\mathbb{C}^{2}$ en $(a, b)$ de la forme

$$
H_{j}(x, y, z)=z^{a_{j}}-\alpha(x, y) B_{j}(x, y)^{l_{j}}
$$

où $\alpha \in A$ et $\alpha(0,0) \neq 0$. Puisque $\operatorname{pgcd}\left(a_{j}, l_{j}\right)=1$, ce facteur est irréductible sur $K$ (voir [23, chap. VIII, $\S 9]$ ).

Comme $a_{j}>1$, de I.4.12 suit que $y=\eta(x)$ est une solution de l'équation, ce qui implique que $\eta(x)$ est constante. Donc, on peut supposer $B_{j}(x, y)=$ $y-b_{j}$ où $b_{j} \in \mathbb{C}$. En plus, on vérifie tout de suite que poids $H_{j}=l_{j}$. Alors, par I.4.11, $l_{j} \geq a_{j}-1$; en particulier,

$$
\frac{k_{j}}{m}=\frac{l_{j}}{a_{j}} \geq 1-\frac{1}{a_{j}} \geq \frac{1}{2} .
$$

Si $k_{j}<m$, on a $l_{j}<a_{j}$; donc, $l_{j}=a_{j}-1$ et

$$
\frac{k_{j}}{m}=\frac{a_{j}-1}{a_{j}} .
$$

En résumé, on a les propriétés suivantes :

i) $2 m \geq k_{1} \geq \cdots \geq k_{r}>0$ et

$$
\sum_{j=1}^{r} \frac{k_{j}}{m} \operatorname{deg}_{y} B_{j}=2 ;
$$

ii) pour $j=1, \ldots, r$, on a l'inégalité suivante

$$
\frac{k_{j}}{m} \geq \frac{1}{2}
$$

iii) si $k_{j} \neq m, 2 m$ alors $B_{j}(x, y)=y-b_{j}, b_{j} \in \mathbb{C}$ et les $b_{j}$ sont tous différents.

iv) si $k_{j}<m$ alors il existe $a_{j} \in \mathbb{N}, a_{j}>1$ tel que $k_{j} / m=\left(a_{j}-1\right) / a_{j}$.

On distingue plusieurs cas.

A) $k_{1}=2 m$. Alors, par i), $r=1$ et $\operatorname{deg}_{y} B_{1}=1$. L'équation est :

$$
\left(y^{\prime}\right)^{m}-B(x)(a(x) y+b(x))^{2 m}=0
$$

où $a, b \in \mathbb{C}[x]$ et $a \neq 0$. 
B) $k_{1}=m$ et $\operatorname{deg}_{y} B_{1}=2$. Par i), $r=1$ et l'équation devient

$$
\left(y^{\prime}\right)^{m}-B(x)\left(a(x) y^{2}+b(x) y+c(x)\right)^{m}=0
$$

où $a, b, c \in \mathbb{C}[x]$ et $a \neq 0$.

C) $k_{1}=k_{2}=m$. Par i), $r=2$ et $\operatorname{deg}_{y} B_{1}=\operatorname{deg}_{y} B_{2}=1$. L'équation est alors

$$
\left(y^{\prime}\right)^{m}-B(x)(a(x) y+b(x))^{m}(c(x) y+d(x))^{m}=0
$$

où $a, b, c, d \in \mathbb{C}[x], a c \neq 0$ et $a d-b c \neq 0$.

D) $k_{1}=m, \operatorname{deg}_{y} B_{1}=1, r \geq 3$ et $k_{j}<m$ pour $2 \leq j \leq r$. D'après i) on obtient la relation

$$
\sum_{j=2}^{r} \frac{k_{j}}{m}=1 \text {. }
$$

Par ii) on a que $r=3$ et $m=2 k_{j}, j=2,3$. Donc l'équation devient

$$
\left(y^{\prime}\right)^{2 n}-B(x)(p(x) y+q(x))^{2 n}\left(y-b_{2}\right)^{n}\left(y-b_{3}\right)^{n}=0
$$

où $n \in \mathbb{N}, b_{2}, b_{3} \in \mathbb{C}, b_{2} \neq b_{3}, p, q \in \mathbb{C}[x], p \neq 0$ et $q(x) / p(x) \neq-b_{2}-b_{3}$.

E) $2 m>k_{1}>m, r \geq 2$ et $k_{j}<m$ pour $2 \leq j \leq r$. D'après i) et iii) on obtient

$$
\sum_{j=1}^{r} \frac{k_{j}}{m}=2 .
$$

Par ii) et i), $r=2$ et $k_{1}+k_{2}=2 m$; c'est-à-dire

$$
k_{1}=m+n \text { et } k_{2}=m-n, n \in \mathbb{N}, 0<n<m .
$$

Par iv) $n$ divise $m$ et l'équation est de la forme

$$
\left(y^{\prime}\right)^{d n}-B(x)\left(y-b_{1}\right)^{n(d+1)}\left(y-b_{2}\right)^{n(d-1)}=0,
$$

où $d, n \in \mathbb{N}, b_{1}, b_{2} \in \mathbb{C}, b_{1} \neq b_{2}$.

F) $k_{j}<m$ pour $j=1, \ldots, r$. Comme dans le cas précédent :

$$
\sum_{j=1}^{r} \frac{k_{j}}{m}=2 .
$$

Par ii), $r=3$ ou 4 . On considère les deux sous-cas suivants.

$\left.\mathrm{F}_{1}\right) r=4$. Alors $m=2 k_{j}, 1 \leq j \leq 4$. L'équation s'écrit sous la forme

$$
\left(y^{\prime}\right)^{2 n}-B(x)\left(y-b_{1}\right)^{n}\left(y-b_{2}\right)^{n}\left(y-b_{3}\right)^{n}\left(y-b_{4}\right)^{n}=0,
$$

où $n \in \mathbb{N}$ et les $b_{j} \in \mathbb{C}$ sont tous différents.

$\left.\mathrm{F}_{2}\right) r=3$. Par iv) on a :

$$
\sum_{j=1}^{3} \frac{a_{j}-1}{a_{j}}=2, a_{1} \geq a_{2} \geq a_{3} \geq 2 .
$$


On voit que $a_{2}=a_{3}=2$ est impossible. Alors $a_{2}>2$, ce qui entraîne $a_{1}<7$. Une discussion directe donne :

$$
a_{1}=6, a_{2}=3, a_{3}=2 ; a_{1}=4, a_{2}=4, a_{3}=2 ; a_{1}=3, a_{2}=3, a_{3}=3 .
$$

On obtient les équations suivantes :

$\left.\mathrm{F}_{21}\right)\left(y^{\prime}\right)^{6 n}-B(x)\left(y-b_{1}\right)^{5 n}\left(y-b_{2}\right)^{4 n}\left(y-b_{3}\right)^{3 n}=0$,

$\left.\mathrm{F}_{22}\right)\left(y^{\prime}\right)^{4 n}-B(x)\left(y-b_{1}\right)^{3 n}\left(y-b_{2}\right)^{3 n}\left(y-b_{3}\right)^{2 n}=0$,

$\left.\mathrm{F}_{23}\right)\left(y^{\prime}\right)^{3 n}-B(x)\left(y-b_{1}\right)^{2 n}\left(y-b_{2}\right)^{2 n}\left(y-b_{3}\right)^{2 n}=0$, où $n \in \mathbb{N}, b_{1}, b_{2}, b_{3} \in \mathbb{C}$ sont différents.

Dans les chapitres suivants on considérera l'intégration de ces équations.

\section{I.5 Solutions algébriques et intégrales premières}

Dans certains cas on peut exprimer la "solution générale" de l'équation (I.1) par la seule relation $R(x, y, \lambda)=0$ où $R$ est un polynôme et $\lambda$ un paramètre. Cela veut dire que les fonctions implicites dans cette relation sont des solutions de l'équation. Plus précisément :

Définition I.5.1. On dit que $R \in \mathbb{C}[x, y, \lambda]$ est une intégrale première ration-nelle de l'équation (I.1) si $R$ est irréductible, dépend effectivement de $y$ et $\lambda$ et si pour toutes $x=\alpha(t)$ holomorphe et non-constante et $y=\beta(t)$ méromorphe au voisinage de 0 telles qu'il existe $\lambda_{0} \in \mathbb{C}$ avec

$$
R\left(\alpha(t), \beta(t), \lambda_{0}\right)=0, \text { pour tout } t \neq 0,
$$

on a que $x=\alpha(t), y=\beta(t)$ est une solution locale.

Observation I.5.2. L'exigence de que $R$ soit irréductible est incluse pour éviter des situations comme $R=(x-\lambda) y$ pour l'équation $y^{\prime}=0$.

Proposition I.5.3. Soit $R \in \mathbb{C}[x, y, \lambda]$ irréductible et qui dépend effectivement de $y$ et $\lambda$. Supposons que pour tous $a, b, \lambda_{0} \in \mathbb{C}$ tels que

$$
R\left(a, b, \lambda_{0}\right)=0 \text { et } R_{y}\left(a, b, \lambda_{0}\right) \neq 0,
$$

la fonction $y=y(x)$ implicite dans $R\left(x, y, \lambda_{0}\right)=0$ avec $y(a)=b$ soit une solution. Alors, $R(x, y, \lambda)$ est une intégrale première rationnelle.

Démonstration. Soient $x=\alpha(t)$ holomorphe et non-constante et $y=\beta(t)$ méromorphe au voisinage de $0 \in \mathbb{C}$ telles qu'il existe $\lambda_{0} \in \mathbb{C}$ avec :

$$
R\left(\alpha(t), \beta(t), \lambda_{0}\right)=0 .
$$

Nous devons prouver :

$$
F\left(\alpha(t), \beta(t), \beta^{\prime}(t) / \alpha^{\prime}(t)\right)=0 .
$$


Premier cas. Supposons que $R_{y}\left(\alpha(t), \beta(t), \lambda_{0}\right) \neq 0$. Pour $t_{1} \in \mathbb{C}$ non-nul et proche de 0 , on a que $R_{y}\left(\alpha\left(t_{1}\right), \beta\left(t_{1}\right), \lambda_{0}\right) \neq 0$. De l'hypothèse on déduit aussitôt que (I.2) est satisfaite au voisinage de $t_{1}$, ce qui complète la preuve dans ce cas.

Deuxième cas. Supposons que $R_{y}\left(\alpha(t), \beta(t), \lambda_{0}\right)=0$. Comme dans le premier cas, il suffit de prouver (I.2) au voisinage d'un point $t_{1} \in \mathbb{C}$ non-nul et proche de 0 . Alors il suffit de prouver (I.2) pour $b:=\beta(0) \in \mathbb{C}$ et $a:=\alpha(0) \in \mathbb{C}$ générique.

Soit $K(x, \lambda)$ le discriminant de $R$ comme polynôme en $y$. Alors $K\left(\alpha(t), \lambda_{0}\right)$ est nul et on peut supposer que l'équation locale au voisinage de $(a, b)$ de l'ensemble $\{K(x, \lambda)=0\}$ est $\lambda=\lambda_{0}$. Nous sommes en mesure d'appliquer I.2.1 au point $\left(a, b, \lambda_{0}\right)$. Il en résulte qu'il existe $y=g(x, u)$ analytique au voisinage de $(a, 0)$ tel que $g(a, 0)=b$ et que

$$
R\left(x, g(x, u), \lambda_{0}+u^{k}\right)=0 \text { où } k \geq 1 ;
$$

de plus, on a $\beta(t)=g(\alpha(t), 0)$. En particulier, $\beta^{\prime}(t)=g_{x}(\alpha(t), 0) \alpha^{\prime}(0)$.

Si $u_{0} \neq 0$ alors $R_{y}\left(a, g\left(a, u_{0}\right), \lambda_{0}+u_{0}^{k}\right) \neq 0$. On en déduit, par hypothèse, que

$$
F\left(x, g\left(x, u_{0}\right), g_{x}\left(x, u_{0}\right)\right)=0 .
$$

Alors, $F\left(x, g(x, 0), g_{x}(x, 0)\right)=0$ et il suffit de faire $x=\alpha(t)$.

Exemple I.5.4. Considérons l'équation différentielle

$$
x^{2}\left(y^{\prime}\right)^{2}+(1-2 x y) y^{\prime}+y^{2}=0 .
$$

Comme on vérifie par I.5.3, elle admet l'intégrale première :

$$
R(x, y, \lambda)=x+\lambda^{2} y+\lambda .
$$

Les droites $R(x, y, \lambda)=0$ sont les tangentes à l'hyperbole $4 x y+1=0$. Observons que les solutions $y=0$ et $y=-1 / 4 x$ ne sont pas comprises dans la relation $R(x, y, \lambda)=0$.

Cet exemple montre qu'en général, la connaissance d'une intégrale première ne donne pas immédiatement toutes les solutions.

Corollaire I.5.5. Soit $R \in \mathbb{C}[x, y, \lambda]$ irréductible qui dépend effectivement de $y$ et $\lambda$. Définissons :

$$
\Phi(x, y, u, v):=v^{m} F(x, y, u / v) .
$$

Alors $R(x, y, \lambda)$ est une intégrale première si et seulement s'il divise

$$
\Phi\left(x, y, R_{x}(x, y, \lambda),-R_{y}(x, y, \lambda)\right) .
$$


Démonstration. Supposons que $R$ soit une intégrale première. Soient $a, b, \lambda_{0} \in \mathbb{C}$ tels que $R\left(a, b, \lambda_{0}\right)=0$ et $R_{y}\left(a, b, \lambda_{0}\right) \neq 0$. Alors la fonction $y=y(x)$ implicite dans $R\left(x, y, \lambda_{0}\right)=0$ avec $y(a)=b$ est une solution. En particulier :

$$
F\left(a, b,-\frac{R_{x}\left(a, b, \lambda_{0}\right)}{R_{y}\left(a, b, \lambda_{0}\right)}\right)=F\left(a, b, y^{\prime}(a)\right)=0 .
$$

Alors,

$$
\Phi\left(a, b, R_{x}\left(a, b, \lambda_{0}\right),-R_{y}\left(a, b, \lambda_{0}\right)\right)=0 .
$$

Le résultat suit du fait que $R$ est irréductible et dépend effectivement de $y$.

La réciproque est analogue en appliquant I.5.3.

Exemple I.5.6. Considérons l'équation différentielle

$$
27 y\left(y^{\prime}\right)^{3}-12 x y^{\prime}+8 y=0 .
$$

On vérifie, par I.5.5, que le polynôme

$$
R(x, y, \lambda)=y^{3}-\lambda(x-\lambda)^{2}
$$

est une intégrale première pour celle-là. On observe aussi que la solution $y=0$ satisfait :

$$
R(x, 0,0)=R_{y}(x, 0,0)=0 .
$$

Proposition I.5.7. Soit $R \in \mathbb{C}[x, y, \lambda]$ irréductible qui dépend effectivement de $y$ et $\lambda$. Supposons qu'il existe un ouvert non-vide $U$ de $\mathbb{C}^{2}$ tel que si $y=y(x)$ est une solution par $q \in U$, il existe $\lambda_{0} \in \mathbb{C}$ tel que $R\left(x, y(x), \lambda_{0}\right)=0$. Alors, $R(x, y, \lambda)$ est une intégrale première.

Démonstration. On peut supposer que $(x, y) \in U$ implique

$$
c_{0}(x, y) D(x, y) \neq 0 .
$$

Alors, pour chaque $q=(a, b) \in U$ il existe une solution régulière $y=y(x)$ par $q$ (voir I.1.4). Soit $c=y^{\prime}(a)$. Par l'hypothèse, il existe $\lambda_{0} \in \mathbb{C}$ tel que :

$$
R\left(a, b, \lambda_{0}\right)=0 \text { et } R_{x}\left(a, b, \lambda_{0}\right)+c R_{y}\left(a, b, \lambda_{0}\right)=0 .
$$

Dans les notations de I.5.5 on a :

$$
\Phi\left(a, b, R_{x}\left(a, b, \lambda_{0}\right),-R_{y}\left(a, b, \lambda_{0}\right)\right)=0 .
$$


En effet, si $R_{y}\left(a, b, \lambda_{0}\right)=0$ alors $R_{x}\left(a, b, \lambda_{0}\right)=0$ et c'est trivial. Si, au contraire, $R_{y}\left(a, b, \lambda_{0}\right) \neq 0$ alors

$$
\begin{aligned}
& \Phi\left(a, b, R_{x}\left(a, b, \lambda_{0}\right),-R_{y}\left(a, b, \lambda_{0}\right)\right) \\
& =\left(-R_{y}\left(a, b, \lambda_{0}\right)\right)^{m} F\left(a, b,-\frac{R_{x}\left(a, b, \lambda_{0}\right)}{R_{y}\left(a, b, \lambda_{0}\right)}\right) \\
& =\left(-R_{y}\left(a, b, \lambda_{0}\right)\right)^{m} F(a, b, c) \\
& =0
\end{aligned}
$$

Cela montre que l'image par la projection $(x, y, \lambda) \mapsto(x, y)$ de l'ensemble algébrique défini par

$$
R(x, y, \lambda)=\Phi\left(x, y, R_{x}(x, y, \lambda),-R_{y}(x, y, \lambda)=0\right.
$$

contient $U$. Donc, cet ensemble est de dimension $>1$. Alors, il coïncide avec l'ensemble $\{R(x, y, \lambda)=0\}$. Il résulte de ceci et de I.5.5 que $R(x, y, \lambda)$ est une intégrale première.

Pour la réciproque de I.5.7 voir proposition II.3.18.

Proposition I.5.8. Soit $R \in \mathbb{C}[x, y, \lambda]$ irréductible qui dépend effectivement de $y$ et $\lambda$. Alors, il existe $F \in \mathbb{C}[x, y, z]$ irréductible et qui dépend effectivement de $z$ tel que $R(x, y, \lambda)$ est une intégrale première de l'équation $F\left(x, y, y^{\prime}\right)=0$.

Démonstration. Soit $T \subset \mathbb{C}^{4}$ l'ensemble défini par

$$
R(x, y, \lambda)=0 \text { et } R_{x}(x, y, \lambda)+z R_{y}(x, y, \lambda)=0 .
$$

C'est une surface algébrique. Soit $U \subset \mathbb{C}^{3}$ l'ensemble défini par :

$$
R(x, y, \lambda)=0 \text { et } R_{y}(x, y, \lambda) \neq 0
$$

Alors $U$ est une variété analytique, connexe, non-vide, de dimension 2 (voir $\left[15\right.$, vol. II E, thm. 19]). Considérons l'application $\varphi: U \rightarrow \mathbb{C}^{4}$ définie par

$$
\varphi(x, y, \lambda)=\left(x, y,-\frac{R_{x}(x, y, \lambda)}{R_{y}(x, y, \lambda)}, \lambda\right) .
$$

On vérifie par le critère jacobien que $\varphi(U) \subset T-\operatorname{Sing} T$. Puisque $U$ est connexe, il existe une composante irréductible $T_{0}$ de $T$ qui contient $\varphi(U)$.

Étant donné que $\operatorname{dim} T_{0}=2$, il existe $F(x, y, z) \in \mathbb{C}[x, y, z]$ irréductible tel que $\left.F\right|_{T_{0}}=0$. Donc,

$$
(x, y, \lambda) \in U \text { implique } F\left(x, y,-\frac{R_{x}(x, y, \lambda)}{R_{y}(x, y, \lambda)}\right)=0
$$


ceci entraîne, en particulier, que $F$ dépend effectivement de $z$.

Soient $a, b, \lambda_{0} \in \mathbb{C}$ tels que :

$$
R\left(a, b, \lambda_{0}\right)=0 \text { et } R_{y}\left(a, b, \lambda_{0}\right) \neq 0 .
$$

Soit $y=y(x)$ la fonction implicite dans $R\left(x, y, \lambda_{0}\right)=0$ avec $y(a)=b$. Alors $\left(x, y(x), \lambda_{0}\right) \in U$. Donc,

$$
\begin{aligned}
0 & =F\left(x, y(x),-\frac{R_{x}\left(x, y(x), \lambda_{0}\right)}{R_{y}\left(x, y(x), \lambda_{0}\right)}\right. \\
& =F\left(x, y(x), y^{\prime}(x)\right) .
\end{aligned}
$$

Pour finir la preuve, il suffit alors d'appliquer I.5.3.

Observation I.5.9. On voit dans la preuve de I.5.8 que $F(x, y, z)$ est obtenu à partir des équations

$$
R(x, y, \lambda)=0, R_{x}(x, y, \lambda)+z R_{y}(x, y, \lambda)=0
$$

par élimination de $\lambda$.

Exemple I.5.10. Prenons $R(x, y, \lambda)=x+\lambda^{2} y+\lambda$. On élimine $\lambda$ entre les équations

$$
x+\lambda^{2} y+\lambda=0 \text { et } 1+\lambda^{2} z=0
$$

et on obtient l'équation de I.5.4.

Définition I.5.11. Soit $C \subset \mathbb{C}^{2}$ une courbe algébrique irréductible. On dit que $C$ est une solution algébrique de l'équation (I.1) si $C$ n'est pas une droite verticale et si tout couple de fonctions $x=\alpha(t), y=\beta(t)$, où $\alpha$ est holomorphe non-constante et $\beta$ méromorphe au voisinage de $0 \in \mathbb{C}$, tel que

$$
(\alpha(t), \beta(t)) \in C \text { si } t \neq 0,
$$

est une solution locale.

Exemple I.5.12. $x y^{\prime}-y-x=0$ est dans la classe FP par I.4.16. Si $a \neq 0$, toute solution locale en $a$ est régulière de la forme $y=x(\log x+c)$, où $\log x$ est une branche du logarithme au voisinage de $a$. Comme une branche du logarithme ne peut pas être algébrique, cette équation n'admet pas de solution algébrique.

Lemme I.5.13. Soit $C$ une courbe algébrique irréductible. Supposons qu'il existe $y=y(x)$ holomorphe au voisinage de $a \in \mathbb{C}$ telle que :

a) $y=y(x)$ est une solution locale en $a$;

b) $(x, y(x)) \in C$ pour $x$ au voisinage de $a$.

Alors, $C$ est une solution algébrique. 
Démonstration. Il est évident que $C$ n'est pas une droite verticale.

Soit $C_{0}$ l'ensemble des points réguliers de $C$ où la tangente n'est pas verticale. L'ensemble $C_{0}$ est connexe. Soit $U \subset C_{0}$ l'ensemble des $q:=$ $(a, b) \in C_{0}$ tels que si $y=\eta(x)$ est l'équation locale de $C$ en $q$; alors $y=\eta(x)$ est une solution locale en $a$. On voit tout de suite que $U$ est ouvert et fermé dans $C_{0}$. Comme $C_{0}$ est dense dans $C$, l'hypothèse implique que $U$ n'est pas vide. Donc $U=C_{0}$.

Soient $x=\alpha(t)$ holomorphe non-constante et $y=\beta(t)$ méromorphe au voisinage de $0 \in \mathbb{C}$ telles que

$$
(\alpha(t), \beta(t)) \in C \text { si } t \neq 0 .
$$
$U$

Il existe $t_{1} \in \mathbb{C}$ proche de 0 tel que $\left(\alpha\left(t_{1}\right), \beta\left(t_{1}\right)\right) \in U$. Par définition de

$$
F\left(\alpha(t), \beta(t), \beta^{\prime}(t) / \alpha^{\prime}(t)\right)=0
$$

pour $t$ au voisinage de $t_{1}$. Cela entraîne immédiatement que $x=\alpha(t), y=$ $\beta(t)$ définissent une solution locale.

Pour la proposition suivante on garde les notations du corollaire I.5.5.

Proposition I.5.14. Soit $f(x, y) \in \mathbb{C}[x, y]$ irréductible et qui dépend effectivement de $y$. Soit $C$ la courbe d'équation $f(x, y)=0$. Alors $C$ est une solution algébrique si et seulement si $f(x, y)$ divise le polynôme

$$
\Phi\left(x, y, f_{x}(x, y),-f_{y}(x, y)\right) .
$$

Démonstration. Elle se déduit de I.5.13 comme dans la preuve de I.5.5

Lemme I.5.15. Soit $R \in \mathbb{C}[x, y, \lambda]$ irréductible et qui dépend effectivement de $y$ et $\lambda$. La condition nécessaire et suffisante pour que $R$ soit une intégrale première est que pour $\lambda_{0} \in \mathbb{C}$ générique, l'équation $R\left(x, y, \lambda_{0}\right)=0$ définisse une courbe de $\mathbb{C}^{2}$ dont toutes les composantes irréductibles soient des solutions algébriques.

Démonstration. Comme $R$ dépend de $y, R\left(x, y, \lambda_{0}\right)$ n'est constant que pour un nombre fini de valeurs de $\lambda_{0}$.

D'autre part, comme $R$ est irréductible et $R_{y} \neq 0$, le sous-ensemble de $\mathbb{C}^{3}$ défini par

$$
R(x, y, \lambda)=R_{y}(x, y, \lambda)=0,
$$

est de dimension $\leq 1$. Alors, pour $\lambda_{0}$ générique, le sous-ensemble de $\mathbb{C}^{2}$ défini par

$$
R\left(x, y, \lambda_{0}\right)=R_{y}\left(x, y, \lambda_{0}\right)=0,
$$


est fini. Cela implique que $R\left(x, y, \lambda_{0}\right)$ n'est pas divisible par le carré d'un polynôme non-constant en $x, y$.

Supposons que, pour $\lambda_{0}$ générique, chaque composante irréductible de la courbe de $\mathbb{C}^{2}$ définie par $R\left(x, y, \lambda_{0}\right)=0$ soit une solution algébrique. Alors, de ce qui précède et de I.5.14 il résulte que $R\left(x, y, \lambda_{0}\right)$ divise le polynôme

$$
\Phi\left(x, y, R_{x}\left(x, y, \lambda_{0}\right),-R_{y}\left(x, y, \lambda_{0}\right)\right) .
$$

Donc l'ensemble de $\mathbb{C}^{3}$ défini par

$$
R(x, y, \lambda)=\Phi\left(x, y, R_{x}(x, y, \lambda),-R_{y}(x, y, \lambda)\right)=0
$$

est de dimension $\geq 2$. L'assertion suit de I.5.5.

Observation I.5.16. Supposons que l'équation (I.1) soit dans la classe FP et qu'elle admette une intégrale première rationnelle $R(x, y, \lambda) \in \mathbb{C}[x, y, \lambda]$.

Soit $p:=(a, b, l) \in \mathbb{C}^{3}$ tel que $R(a, b, l)=0$. Si $R_{y}(a, b, l)=0$ et $R_{x}(a, b, l) \neq 0$, le germe $\Sigma$ d'ensemble analytique en $(a, b)$ défini par $R(x, y, l)=0$ est non-singulier et à tangente verticale en $(a, b)$. Par la définition I.5.1, $\Sigma$ est le support d'une solution par $(a, b)$. Alors, il existe une solution ramifiée par $(a, b)$ (voir I.3.13). Donc, si $a$ est générique,

$$
R(a, b, l)=R_{y}(a, b, l)=0 \text { implique } R_{x}(a, b, l)=0 .
$$

D'après la Nullstellensatz (voir[15]) on en déduit qu'il existe $m \in \mathbb{N}$ tel que :

$$
R_{x}(x, y, \lambda)^{m}=A(x, y, \lambda) R(x, y, \lambda)+B(x, y, \lambda) R_{y}(x, y, \lambda)
$$

où $A, B$ sont des polynômes en $y, \lambda$ à coefficients des fonctions rationnelles de $x$. Cette condition nécessaire, pour que l'équation qui admet l'intégrale première $R(x, y, \lambda)$ soit dans la classe $\mathrm{FP}$, n'est même pas suffisante pour qu'elle soit dans la classe FP faible : en effet, il suffit de regarder l'équation

$$
8\left(y^{\prime}\right)^{3}-27 y=0
$$

dont une intégrale première est

$$
R(x, y, \lambda)=(x+\lambda)^{3}-y^{2} .
$$




\section{Chapitre II}

\section{Équations différentielles algébriques et feuilletages}

\section{II.1 Introduction}

Dans ce chapitre nous reprenons l'étude des équations polynomiales sous un angle un peu plus général. Comme au chapitre I on se donne $F \in$ $\mathbb{C}[x, y, z]$ irréductible qui dépend effectivement de $z$,

$$
F(x, y, z)=\sum_{k=0}^{m} c_{k}(x, y) z^{m-k}, m \geq 1, c_{0}(x, y) \neq 0
$$

et $D \in \mathbb{C}[x, y]$ désigne son discriminant comme polynôme en $z$.

On se donne aussi $P, Q \in \mathbb{C}[x, y, z]$ premiers entre eux et tels que $F$ ne divise pas $Q$. Cela implique que l'équation

$$
F(x, y, z)=Q(x, y, z)=0
$$

définit un sous-ensemble algébrique de $\mathbb{C}^{3}$ de dimension pure 1 (éventuellement vide). On définit $\Delta \subset \mathbb{C}^{2}$ comme étant la réunion de l'adhérence de l'image de cet ensemble par la projection $(x, y, z) \mapsto(x, y)$ avec l'ensemble défini par $D(x, y)=0$. Alors $\Delta$ est un sous-ensemble algébrique de dimension $\leq 1$.

On considère l'équation différentielle :

$$
\left\{\begin{array}{l}
P(x, y, z) d x-Q(x, y, z) d y=0 \\
F(x, y, z)=0
\end{array}\right\}
$$

(l'équation $F\left(x, y, y^{\prime}\right)=0$ correspond au cas particulier où $P=z, Q=1$ ).

Définition II.1.1. Une solution locale en $a \in \mathbb{C}$ de (II.1) est un couple de fonctions $x=\alpha(t), y=\beta(t)$ où $\alpha$ est holomorphe et non-constante et 
$\beta$ est méromorphe au voisinage de $0 \in \mathbb{C}$ telles que $\alpha(0)=$ a et que les équations en $z$

$$
P(\alpha(t), \beta(t), z) \alpha^{\prime}(t)-Q(\alpha(t), \beta(t), z) \beta^{\prime}(t)=0, F(\alpha(t), \beta(t), z)=0
$$

ont une racine en commun pour tout $t \neq 0$ au voisinage de 0.

Exemple II.1.2. a) $z^{2} d x-d y=0, z^{2}+\left(y-x^{2}\right) z-2 x=0$. Une solution locale en 0 est $x=t, y=t^{2}$. Observons qu'il n'existe pas de fonction (uniforme) $z=\gamma(t)$ qui soit solution des deux équations de (II.1.1) pour $t \neq 0$.

b) $d x-d y=0, x z-1=0$. Une solution locale en 0 est $x=t, y=t$. Observons qu'il n'existe pas de racine commune aux équation $1-1=$ $0, t z-1=0$ si $t=0$. c) $(x-y) d x-\left(z^{3}+x z-x^{2} z-x^{2}\right) d y=0$, $z^{3}+y z-x^{2} z-x^{2}=0$. On a les solutions locales en 0 paramétrée par $x=t^{2}, y=t^{2}$ avec

$$
z=t^{2} \quad \text { ou } \quad z=\frac{1}{2}\left(-t^{2} \pm t \sqrt{t^{2}-4}\right)
$$

(observons que $z^{3}+x z-x^{2} z-x^{2}=(z-x)\left(z^{2}+x z+x\right)$ ce qui permet de montrer facilement qu'elles sont bien des solutions); dans le deuxième cas les exposants des termes non-nuls de la série de Taylor de $z=\gamma(t)$ en 0 ne sont pas tous multiples de 2 .

Lemme II.1.3. Si $x=\alpha(t), y=\beta(t)$ est une solution locale de (II.1), alors il existe $t=t(s)$, holomorphe et non-constante au voisinage de $0 \in \mathbb{C}$, avec $t(0)=0$, et $z=\gamma(s)$, méromorphe au voisinage de $0 \in \mathbb{C}$, telles que

$$
P(\alpha(s), \beta(s), \gamma(s)) \alpha^{\prime}(s)-Q(\alpha(s), \beta(s), \gamma(s)) \beta^{\prime}(s)=F(\alpha(s), \beta(s), \gamma(s))=0
$$

pour tout $s \neq 0$ au voisinage de 0 , ò̀ $\alpha(s):=\alpha(t(s)), \beta(s):=\beta(t(s))$.

Démonstration. Soit $E_{0} \subset D^{*} \times \mathbb{C}$ l'ensemble analytique défini par les équations

$$
P(\alpha(t), \beta(t), z) \alpha^{\prime}(t)-Q(\alpha(t), \beta(t), z) \beta^{\prime}(t)=F(\alpha(t), \beta(t), z)=0,
$$

où $D$ désigne un petit disque de centre $0 \in \mathbb{C}$ et $D^{*}:=D-\{0\}$.

Il est clair que l'adhérence $E$ de $E_{0}$ dans $D \times \mathbb{P}_{1}$ est aussi un ensemble analytique. Comme $F$ est irréductible, $F(\alpha(t), \beta(t), z)$ n'est pas identiquement nul. Alors, $\operatorname{dim} E \leq 1$. De la définition II.1.1 il résulte qu'il existe un germe irréductible $S \subset E$ d'ensemble analytique de dimension 1 en un point $u \in\{0\} \times \mathbb{P}_{1}$ tel que $S-\{u\} \subset D^{*} \times \mathbb{C}$. On définit $t=t(s), z=\gamma(s)$ comme étant une paramétrisation de ce germe définie au voisinage de $0 \in \mathbb{C}$ (voir [4]). 
Les notions de solution par $(a, b)$, solutions équivalentes, pente d'une solution, solution uniforme, solution régulière, définies au $\S 1 \mathrm{du}$ chapitre I se généralisent immédiatement à l'équation (II.1). La notion de solution ramifiée est généralisée de la manière suivante :

Une solution locale en $a \in \mathbb{C}$ est non-ramifiée si pour toute paramétrisation de cette solution de la forme

$$
x=\alpha(t)=a+t^{M}, y=\beta(t)
$$

et pour toute $z=\gamma(t)$ méromorphe au voisinage de $0 \in \mathbb{C}$ telle que:

$$
P(\alpha(t), \beta(t), \gamma(t)) \alpha^{\prime}(t)-Q(\alpha(t), \beta(t), \gamma(t)) \beta^{\prime}(t)=F(\alpha(t), \beta(t), \gamma(t))=0,
$$

pour tout $t \neq 0$ au voisinage de 0 , les exposants des termes non-nuls des séries de Laurent de $\beta(t)$ et $\gamma(t)$ sont des multiples de $M$ (c'est-à-dire, y et $z$ sont des fonctions de $x)$. Une solution locale en $a \in \mathbb{C}$ est ramifiée si elle n'est pas non-ramifiée.

L'exemple II.1.2c montre que $z=\gamma(t)$ n'est pas nécessairement unique. Toute solution non-ramifiée est uniforme au sens du $\S 1$ du chapitre I. Mais, contrairement à ce qui arrive dans le cas polynomial, une solution uniforme peut être ramifiée (voir exemple II.1.2).

Lemme II.1.4. Toute solution par $(a, b) \in \mathbb{C}^{2}$ de pente $\infty$ est ramifiée.

Démonstration. Voir lemme I.3.13

Les notions de point de ramification, classe de Fuchs-Painlevé, classe de Fuchs-Painlevé faible définies au $\S 4$ du chapitre I, se généralisent aussi au cas de l'équation (II.1), ainsi que celle de solution algébrique de la définition I.5.11.

On va donner une définition légèrement plus générale d'intégrale première rationnelle mais qui est équivalente à celle qu'on a traité dans le cas de l'équation $F\left(x, y, y^{\prime}\right)=0$ (voir définition I.5.1 et lemme I.5.15).

Définition II.1.5. Soit $R \in \mathbb{C}[x, y, \lambda]$ irréductible et qui dépend effectivement de $y$ et de $\lambda$. On dit que $R$ est une intégrale première rationnelle de (II.1) si pour tout $\lambda_{0} \in \mathbb{C}$ générique, $R\left(x, \overline{\left.x, \lambda_{0}\right)=0 \text { définit une courbe }}\right.$ algébrique de $\mathbb{C}^{2}$ dont toutes les composantes irréductibles sont des solutions algébriques de (II.1).

Exemple II.1.6. Considérons $d x-z d y=0, y z-x=0$. Le polynôme $R=y-\lambda x$ est une intégrale première mais $y=0$ n'est pas une solution.

En général, le sous-ensemble $E \subset \mathbb{C}^{3}$ défini par l'équation

$$
P(x, y, z)=Q(x, y, z)=F(x, y, z)=0
$$

est fini. Si, par contre, l'ensemble est de dimension $\geq 1$, alors tout couple de fonctions $x=\alpha(t), y=\beta(t)$ méromorphes au voisinage de $0 \in \mathbb{C}$ avec $\alpha$ 
holomorphe et non-constante tel que $(\alpha(t), \beta(t))$ appartient à la projection de $E$ sur $\mathbb{C}^{2}$ pour $t \neq 0$, fournit une solution locale de (II.1). Ceci justifie la définition suivante :

Définition II.1.7. Une solution locale $x=\alpha(t), y=\beta(t)$ de (II.1) est



$$
P(\alpha(t), \beta(t), z)=0, Q(\alpha(t), \beta(t), z)=0, F(\alpha(t), \beta(t), z)=0
$$

ont une racine en commun.

Considérons maintenant une équation différentielle de la forme

$$
\left\{\begin{array}{l}
P_{1}(x, y, z) d x-Q_{1}(x, y, z) d y=0 \\
F(x, y, z)=0
\end{array}\right\}
$$

où $P_{1}$ et $Q_{1}$ sont premiers entre eux et $F$ ne divise pas $Q_{1}$.

Définition II.1.8. Les équations (II.1) et (II.2) sont équivalentes si F divise $P Q_{1}-Q P_{1}$.

Lemme II.1.9. Si (II.1) et (II.2) sont équivalentes alors toute solution locale non-triviale de l'une est une solution locale de l'autre.

Démonstration. Supposons que $F$ divise $P Q_{1}-Q P_{1}$. Soit $x=\alpha(t), y=$ $\beta(t)$ une solution locale non-triviale de (II.1). Notons $T$ l'image de l'ensemble

$$
\left\{(x, y, z) \in \mathbb{C}^{3}: F(x, y, z)=P(x, y, z)=Q(x, y, z)=0\right\}
$$

par la projection $(x, y, z) \mapsto(x, y)$ sur $\mathbb{C}^{2}$. Comme cet ensemble est de dimension $\leq 1$, on a que le complémentaire de $T$ dans son adhérence Zariski dans $\mathbb{P}_{1}^{2}$ est fini. Il en résulte que $(\alpha(t), \beta(t)) \notin T$ pour tout $t \neq 0$ au voisinage de 0 . De ceci et de la définition II.1.1 on déduit que $x=\alpha(t), y=$ $\beta(t)$ est une solution locale de (II.2).

Exemple II.1.10. a) Prenons $F=z, P=y+z, Q=y, P_{1}=1, Q_{1}=1$. Alors $F$ divise $P Q_{1}-Q P_{1}$ et $y=0$ est une solution de (II.1) mais pas de (II.2).

b) Les équations

$$
z d x-d y=0, x+y+z^{2}=0 \text { et } z d x+d y=0, x+y+z^{2}=0
$$

ont les mêmes solutions mais elles ne sont pas équivalentes.

Proposition II.1.11. Soit $D \subset \mathbb{C}$ un disque ouvert de centre $0 \in \mathbb{C}$ et soient $\alpha: D \rightarrow \mathbb{C}, \beta: D^{*}:=D-\{0\} \rightarrow \mathbb{C}$ holomorphes, avec $\alpha$ nonconstante, où 0 est, au plus, un pôle de $\beta$. Supposons qu'il existe $t_{0} \in D^{*}$ tel que pour tout $t \neq t_{0}$ assez proche de $t_{0}$, les équations en $z$

$$
P(\alpha(t), \beta(t), z) \alpha^{\prime}(t)-Q(\alpha(t), \beta(t), z) \beta^{\prime}(t)=0, F(\alpha(t), \beta(t), z)=0
$$


ont une racine en commun. Alors $x=\alpha(t), y=\beta(t)$ est une solution locale de (II.1) en $a=\alpha(0)$.

Démonstration. Considérons les expressions

$$
\begin{gathered}
M(t, z):=P(\alpha(t), \beta(t), z) \alpha^{\prime}(t)-Q(\alpha(t), \beta(t), z) \beta^{\prime}(t), \\
N(t, z):=F(\alpha(t), \beta(t), z),
\end{gathered}
$$

avec $t \in D^{*}, z \in \mathbb{C}$. Ce sont des polynômes en $z$, analytiques en $t$, avec $N$ non-identiquement nul (parce que $F(x, y, z)$ est irréductible). Soit $S \subset D^{*}$ l'ensemble des zéros du coefficient du terme de plus haut degré en $z$ dans $N$. Alors $S$ est un sous-ensemble discret et fermé de $D$.

Soit $T \subset D^{*} \times \mathbb{C}$ le sous-ensemble analytique défini par

$$
M(t, z)=N(t, z)=0 .
$$

Alors, si $p: D^{*} \times \mathbb{C} \rightarrow D^{*}$ est la projection canonique, sa restriction au sousensemble analytique $T \cap p^{-1}\left(D^{*}-S\right)$ est propre. Donc $p\left(T \cap p^{-1}\left(D^{*}-S\right)\right)$ est un sous-ensemble analytique de $D^{*}-S$. Par hypothèse cet ensemble contient un ouvert non-vide; d'où que $p(T) \supset D^{*}-S$, ce qui entraîne tout de suite l'assertion.

Dans la suite de ce chapitre on montrera qu'on peut réduire l'étude globale de (II.1) à celle d'un feuilletage sur une surface fibrée. Comme on le verra l'équation (II.1) est dans la classe FP si et seulement si le feuilletage associé est génériquement transverse aux fibres. On profitera du théorème de Jouanolou [21] pour prouver que (II.1) possède une infinité de solutions algébriques si et seulement si elle admet une intégrale première rationnelle. Dans l'observation II.3.17 on corrige la condition nécessaire 4 de [29].

\section{II.2 L'équation polynomiale associée}

On peut ramener (II.1) à une équation polynomiale en éliminant $z$ des équations :

$$
y^{\prime}=\frac{P(x, y, z)}{Q(x, y, z)}, F(x, y, z)=0 .
$$

Plus précisément, notons $T_{0} \subset \mathbb{C}^{4}$ l'ensemble algébrique de dimension pure 2 défini par les équations

$$
P(x, y, z)-w Q(x, y, z)=0, F(x, y, z)=0,
$$

et $S_{0} \subset \mathbb{C}^{3}$ la surface irréductible définie par $F(x, y, z)=0$; désignons par $U$ l'ensemble des points réguliers de $S_{0}$. L'application

$$
U-\{Q=0\} \rightarrow \mathbb{C}^{4},(x, y, z) \mapsto(x, y, z, P(x, y, z) / Q(x, y, z))
$$


est un plongement. Comme l'ensemble $\{Q(x, y, z)=F(x, y, z)=0\}$ est de dimension $\leq 1$, l'image de ce plongement est un sous-ensemble dense de $T_{0}$. Alors, $T_{0}$ est irréductible.

On en déduit qu'il existe $G \in \mathbb{C}[x, y, w]$ irréductible tel que :

$$
(x, y, z, w) \in T_{0} \text { implique } G(x, y, w)=0 .
$$

Comme l'image de $T_{0}$ par la projection $(x, y, z, w) \mapsto(x, y)$ est dense dans $\mathbb{C}^{2}$, le polynôme $G$ dépend effectivement de $w$. Cela montre aussi que l'image de $T_{0}$ par la projection $(x, y, z, w) \mapsto(x, y, w)$ n'est pas contenue dans une courbe algébrique, ce qui implique que $G$ est déterminé à une constante multiplicative non-nulle près.

Définition II.2.1. On dira que $G\left(x, y, y^{\prime}\right)=0$ est une équation polynomiale associée à (II.1).

Si $Y$ est un espace analytique et $p \in Y$ est un point régulier, $T_{p}(Y)$ désigne l'espace tangent de $Y$ en $p$.

Proposition II.2.2. On a les assertions suivantes :

a) Toute solution locale de (II.1) est une solution locale de $G\left(x, y, y^{\prime}\right)=0$.

b) Il existe un ouvert de Zariski non-vide $W \subset \mathbb{C}^{2}$ tel que toute solution de $G\left(x, y, y^{\prime}\right)=0$ par $q \in W$ est régulière et est une solution de (II.1).

c) Si l'équation (II.1) est dans la classe FP, alors $G\left(x, y, y^{\prime}\right)=0$ est aussi dans la classe FP.

Démonstration. a) C'est une conséquence immédiate de la définition II.1.1. b) Soit $R_{0} \subset \mathbb{C}^{3}$ la surface d'équation $G(x, y, w)=0$. Il existe un sousensemble algébrique $Z$ de $R_{0}$ tel que $\operatorname{dim} Z \leq 1$ et que si $(x, y, w) \in R_{0}-Z$, alors il existe $z \in \mathbb{C}$ tel que $(x, y, z, w) \in T_{0}$, où $T_{0}$ est l'ensemble défini ci-dessus. Soit $\Sigma$ un sous-ensemble algébrique propre de $\mathbb{C}^{2}$ qui contient l'image de $Z$ par la projection $(x, y, w) \mapsto(x, y)$. Soit $W \subset \mathbb{C}^{2}-\Sigma$ un ouvert de Zariski non-vide tel que toute solution de $G\left(x, y, y^{\prime}\right)=0$ par $q \in W$ est régulière (corollaire I.1.4). Ce $W$ satisfait aux conditions voulues.

c) Supposons que $G\left(x, y, y^{\prime}\right)=0$ ne soit pas dans la classe FP ; notons $Y$ l'adhérence de $\mathbb{C}^{2}-W$ dans $\mathbb{P}_{1}^{2}$ où $W$ est l'ouvert de b) : c'est un sousensemble analytique de $\mathbb{P}_{1}^{2}$ de dimension (pas forcément pure) $\leq 1$. Pour $a \in \mathbb{C}$ générique, si $p=(a, b)$ appartient à $Y(b \in \mathbb{C} \cup\{\infty\})$, on peut supposer que $\operatorname{dim}_{p} Y=1$, que $p$ est un point régulier de $Y$ et que $T_{p}(Y)$ n'est pas verticale. Alors, si $x=\alpha(t), y=\beta(t)$ est une solution ramifiée par $p$, on aura que $(\alpha(t), \beta(t)) \in W$ pour tout $t \neq 0$ assez proche de 0 . D'après b) et la proposition II.1.11, $x=\alpha(t), y=\beta(t)$ est une solution locale de (II.1). Alors (II.1) n'est pas dans la classe FP, ce qui termine la preuve. 
Corollaire II.2.3. On a les assertions suivantes :

a) Toute solution algébrique de (II.1) est une solution algébrique de l'équation $G\left(x, y, y^{\prime}\right)=0$.

b) Il existe un nombre fini $C_{1}, C_{2}, \ldots, C_{k}$ de courbes algébriques et irréductibles dans $\mathbb{C}^{2}$ telles que si $C$ est une solution algébrique de $G\left(x, y, y^{\prime}\right)=$ 0 avec $C \neq C_{j}, j=1,2, \ldots, k$, alors $C$ est une solution algébrique de (II.1).

Démonstration. a) Elle découle immédiatement de II.2.2a.

b) Soient $C_{1}, C_{2}, \ldots, C_{k}$ les composantes irréductibles de $\mathbb{C}^{2}-W$, où $W$ est l'ouvert donné par II.2.2b. Soit $C \neq C_{1}, C_{2}, \ldots, C_{k}$ une solution algébrique de $G\left(x, y, y^{\prime}\right)=0$. Alors $C \cap\left(\mathbb{C}^{2}-W\right)$ est un ensemble fini. Soit $x=$ $\alpha(t), y=\beta(t)$ une solution locale de $G\left(x, y, y^{\prime}\right)=0$ contenue dans $C$. Alors, $(\alpha(t), \beta(t)) \in W$ si $t \neq 0$. De ceci et de II.2.2b il résulte que $x=$ $\alpha(t), y=\beta(t)$ est une solution locale de (II.1) (voir proposition II.1.11).

Exemple II.2.4. On considère l'équation

$$
\left\{\begin{array}{l}
d x-z d y=0 \\
y z^{2}-x=0
\end{array}\right\}
$$

L'équation associée est donc

$$
\left(y^{\prime}\right)^{2} x-y=0 .
$$

Alors $y=0$ est une solution algébrique de celle-ci qui n'est pas solution de l'équation donnée.

Corollaire II.2.5. Soit $R \in \mathbb{C}[x, y, \lambda]$. Alors $R$ est une intégrale première de (II.1) si et seulement si $R$ est une intégrale première de $G\left(x, y, y^{\prime}\right)=0$.

Démonstration. Elle découle de la définition II.1.5, du lemme I.5.15 et du corollaire II.2.3.

Ce qui précède permet d'appliquer les résultats du chapitre I à l'équation (II.1). En particulier on a la proposition suivante.

Proposition II.2.6. Soit $R \in \mathbb{C}[x, y, \lambda]$ irréductible et qui dépend effectivement de $y$ et de $\lambda$. Supposons qu'il existe un ouvert non-vide $U$ de $\mathbb{C}^{2}$ tel que si $y=y(x)$ est une solution régulière de (II.1) par $q \in U$, il existe $\lambda_{0} \in \mathbb{C}$ tel que $R\left(x, y(x), \lambda_{0}\right)=0$. Alors, $R$ est une intégrale première.

Démonstration. Cela résulte des propositions I.5.7 et II.2.2 et du corollaire II.2.5.

Pour la réciproque de la proposition II.2.6 voir proposition II.3.18 plus bas. 


\section{II.3 Construction du feuilletage}

Il est bien connu, quoique ce soit un résultat profond et difficile à démontrer, que si $Z$ est une variété algébrique projective avec ensemble de singularités $\Sigma$, il existe une variété projective lisse $\widetilde{Z}$ et une application analytique birationnelle $\pi: \widetilde{Z} \rightarrow Z$ qui induit, par restriction, un isomorphisme

$$
\pi: \widetilde{Z}-\pi^{-1}(\Sigma) \longrightarrow Z-\Sigma .
$$

Ce résultat a été démontré par Hironaka ([19]) et la variété $\widetilde{Z}$ s'appelle une désingularisation de $Z$. Dans le cas où la variété est une surface, c'està-dire de dimension 2 , on peut faire une démonstration différente de celle d'Hironaka et plus élémentaire : voir [2, chap.III, §6]. Le cas où $Z$ est une courbe est classique et se trouve dans plusieurs livres de géométrie algébrique; pour une preuve assez constructive on peut regarder [2, chap. II, §7] où on traite le cas (qui nous suffit pour les application que l'on a en vue) d'une courbe contenue dans une surface lisse; une preuve très directe mais intrinsèque consiste à prendre la normalisation de la courbe (indépendamment du fait d'être plongée dans une surface lisse). Finalement, il faut dire que la désingularisation n'est pas unique (à isomorphisme près) en général pour des variétés de dimension supérieure ou égale à 2 ; en revanche, il n'y a qu'une désingularisation, à isomorphisme près, pour chaque courbe algébrique : celle-ci est appelée quelquefois le modèle lisse de la courbe.

On appellera surface fibrée une surface analytique complexe compacte, connexe, non-singulière $X$ munie d'une application holomorphe surjective $h: X \rightarrow B$ où $B$ est une surface de Riemann.

Si $b \in B$ est une valeur régulière de $h$ on dira que $h^{-1}(b)$ est une fibre régulière.

Si $C$ est une courbe algébrique (affine ou projective) on appellera genre de $C$ le genre de la désingularisation projective de $C$.

On identifiera toujours la droite projective complexe $\mathbb{P}_{1}=\mathbb{P}_{1}(\mathbb{C})$ avec $\mathbb{C} \cup\{\infty\}$. Alors,

$$
\mathbb{C}^{k} \subset \mathbb{P}_{1} \times \cdots{ }^{k} \cdots \times \mathbb{P}_{1}=\mathbb{P}_{1}^{k}
$$

Définition II.3.1. Soit $x=\alpha(t), y=\beta(t)$ une solution de (II.1) par $q:=(a, b) \in \mathbb{P}_{1}^{2}$.

a) Le germe en $q$ du sous-ensemble analytique de $\mathbb{P}_{1}^{2}$ défini par l'image (ou graphe) de cette solution, sera appelé le support de la solution.

b) Si $\gamma(t)$ est méromorphe au voisinage de $\overline{0 \in \mathbb{C}}$ et si

$$
P(\alpha(t), \beta(t), \gamma(t)) \alpha^{\prime}(t)-Q(\alpha(t), \beta(t), \gamma(t)) \beta^{\prime}(t)=F(\alpha(t), \beta(t), \gamma(t))=0
$$

pour tout $t \neq 0$ au voisinage de 0 , alors le germe en $p:=(a, b, c)$, où $c=\gamma(0)$, du sous-ensemble analytique de $\mathbb{P}_{1}^{3}$, image de $x=\alpha(t), y=$ $\beta(t), z=\gamma(t)$, sera appelé un relèvement du support de la solution. 
Si $\Sigma$ est le support d'une solution par $q=(a, b)$, alors l'application locale

$$
(\Sigma, q) \rightarrow(\mathbb{C}, a),(x, y) \mapsto x
$$

est finie. D'après les définitions on a le résultat suivant.

Lemme II.3.2. Une solution de (II.1) est non-ramifiée si et seulement si l'application $(x, y, z) \mapsto x$ restreinte à chaque relèvement du support de cette solution est de degré 1.

Soit $S_{0} \subset \mathbb{C}^{3}$ la surface algébrique irréductible d'équation $F(x, y, z)=0$. Soit $S$ l'adhérence de $S_{0}$ dans $\mathbb{P}_{1}^{3}$. C'est une surface projective irréductible.

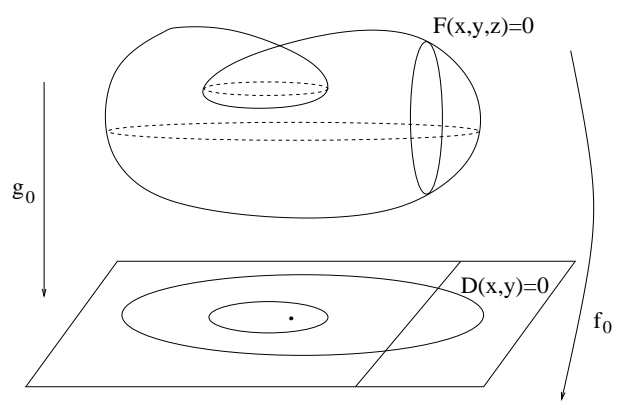

Figure II.1

Soit $\pi: X \rightarrow S$ une désingularisation de $S$. Considérons les applications holomorphes

$$
f_{0}: S_{0} \rightarrow \mathbb{C}, f_{0}(x, y, z)=x \text { et } g_{0}: S_{0} \rightarrow \mathbb{C}^{2}, g_{0}(x, y, z)=(x, y) .
$$

Alors $f_{0}, g_{0}$ se prolongent à des applications $f: S \rightarrow \mathbb{P}_{1}$ et $g: S \rightarrow \mathbb{P}_{1}^{2}$ respectivement; posons $f^{\prime}:=f \circ \pi, g^{\prime}:=g \circ \pi$.

On sait que $f^{\prime}$ se factorise à travers un revêtement ramifié $\varphi: B \rightarrow \mathbb{P}_{1}$ et définit une surface fibrée $h: X \rightarrow B$ dont les fibres sont connexes ([2, chap. III, $\S 11$, cor. 11.5]).

Considérons, finalement, les ouverts de Zariski non-vides :

$$
V:=g_{0}^{-1}\left(\mathbb{C}^{2}-\Delta\right), U:=\pi^{-1}(V) .
$$

On a le diagramme commutatif : 




Considérons la 1-forme holomorphe sur $\mathbb{C}^{3}$ définie par

$$
\omega:=P(x, y, z) d x-Q(x, y, z) d y,
$$

où $P, Q$ sont les polynômes de l'équation (II.1).

Lemme II.3.3. On a

a) $\left.\pi\right|_{U}: U \rightarrow V$ est un isomorphisme.

b) $\left.\omega\right|_{V}$ est holomorphe et jamais nulle.

Démonstration. Par définition de $\Delta, F_{z}$ ne s'annule jamais sur $V$. Alors, les points de $V$ sont non-singuliers dans $S$, ce qui prouve a).

D'autre part, $\left.\omega\right|_{V}$ est holomorphe. Si $a \in V$, on peut prendre $x, y$ comme coordonnées locales dans $V$ au voisinage de $p$. Puisque $Q(p) \neq 0$, il en suit

$$
\left.\omega\right|_{V}(p) \neq 0 .
$$

Lemme II.3.4. Pour $a \in \mathbb{C}$ générique, $\pi: f^{\prime-1}(a) \rightarrow C_{a}$ est une application birationnelle où $C_{a} \subset \mathbb{C}^{2}$ est la courbe définie par $F(a, y, z)=0$.

Démonstration. $S-S_{0}$ est composé d'un nombre fini de courbes irréductibles. On peut supposer qu'aucune de ces courbes n'est contenue dans $f^{-1}(a)$. Alors, $f^{-1}(a)$ est l'adhérence de $f_{0}^{-1}(a)$.

D'autre part, on peut supposer que $f^{\prime-1}(a)$ ne contient aucune composante irréductible de $X-U$. Cela implique que $\pi: f^{\prime-1}(a) \rightarrow f^{-1}(a)$ est un morphisme birationnel par le lemme II.3.3a.

Proposition II.3.5. Pour a $\in \mathbb{C}$ générique le genre de chaque composante irréductible de la courbe $F(a, y, z)=0$ est égal au genre de la fibre générique de $h$. En particulier, toutes ces composantes ont le même genre g qui ne dépend pas de a.

Démonstration. En effet, par le lemme II.3.4 les désingularisées projectives de ces composantes sont des fibres génériques de $h$. 
Définition II.3.6. L'entier $g \geq 0$ est appelé le genre de l'équation (II.1).

Exemple II.3.7. Revenons à l'équation binomiale de l'exemple I.4.17. Dans les cas A),B) et C) on voit tout de suite que les composantes irréductibles de la courbe $F(a, y, z)=0$ sont toutes rationnelles, pour $a \in \mathbb{C}$ générique. Donc, le genre de ces équations est 0 . Dans les cas D) et E) on a encore genre 0 par la formule de Hurwitz. Cette même formule nous permet de montrer qu'on a genre 1 dans les cas restants.

Considérons $\omega=P(x, y, z) d x-Q(x, y, z) d y$ comme 1-forme méromorphe $\operatorname{sur} \mathbb{P}_{1}^{3}$. Alors $\pi^{*}(\omega)$ est une 1-forme méromorphe non-nulle sur $X$. L'équation de Pfaff $\pi^{*}(\omega)=0$ définit un feuilletage analytique de $X$ à singularités isolées (voir annexe $\$ 3$ ) ; il est différent du feuilletage défini par la fibration $h$. On note $\mathcal{F}(P, Q)$ ce feuilletage qui est associé à l'équation (II.1).

Si $W$ est un espace analytique irréductible, on note $\mathbb{C}(W)$ le corps des fonctions méromorphes sur $W$.

Théorème II.3.8. On a les assertions suivantes.

a) Tout feuilletage analytique à singularités isolées sur $X$, différent de celui défini par $h$, est de la forme $\mathcal{F}(P, Q)$ pour $P, Q \in \mathbb{C}[x, y, z]$.

b) Les équations (II.1) et (II.2) définissent le même feuilletage sur $X$ si et seulement si elles sont équivalentes.

Démonstration. a) Comme $X$ est une variété algébrique, tout feuilletage de $X$ est défini par une équation de Pfaff $\alpha=0$ où $\alpha$ est une 1-forme méromorphe non-nulle sur $X$ (voir annexe $\S 3$ ).

Soient $\xi, \eta \in \mathbb{C}(X)$ les fonctions obtenues par composition de $g^{\prime}$ avec les projections canoniques de $\mathbb{P}_{1}^{2}$ sur le premier et le deuxième facteur respectivement. Comme $g^{\prime}$ est dominante, $\xi$ et $\eta$ sont algébriquement indépendantes. Alors,

$$
\alpha=u d \xi-v d \eta, u, v \in \mathbb{C}(X) .
$$

Puisque $\pi$ est birationnelle, il existe $A, B \in \mathbb{C}[x, y, z]$ tel que $F$ ne divise pas $C$ et

$$
u=\left(\frac{A(x, y, z)}{C(x, y, z)}\right) \circ \pi, v=\left(\frac{B(x, y, z)}{C(x, y, z)}\right) \circ \pi .
$$

De plus, comme $\alpha \neq 0$, le polynôme $F$ ne divise pas $A$ ou $B$.

Soit $R:=\operatorname{pgcd}(A, B)$. Posons

$$
P(x, y, z):=\frac{A(x, y, z)}{R(x, y, z)}, Q(x, y, z):=\frac{B(x, y, z)}{R(x, y, z)} .
$$

Considérons la 1-forme rationnelle sur $\mathbb{P}_{1}^{3}$ définie par

$$
\omega:=P(x, y, z) d x-Q(x, y, z) d y
$$

on voit que

$$
\pi^{*}(\omega) \wedge \alpha=0 \text { et } \pi^{*}(\omega) \neq 0 .
$$


Donc, $\alpha=0$ et $\pi^{*}(\omega)=0$ définissent le même feuilletage analytique de $X$ à singularités isolées (voir annexe $\S 3$ ); de plus $F$ ne divise pas $Q$, car ce feuilletage est différent de celui défini par $h$. Pour ces polynômes $P, Q$ le feuilletage $\mathcal{F}(P, Q)$ associé à (II.1) est identique à celui défini par $\alpha=0$.

b) Il suit de la définition II.1.8 et (à nouveau) du fait que deux équations de Pfaff $\alpha=0, \beta=0$ définissent le même feuilletage à singularités isolées si et seulement si $\alpha \wedge \beta=0$.

Définition II.3.9. Un germe $\Lambda$ d'ensemble analytique irréductible de dimension 1 en $p \in X$ est une séparatrice locale de $\mathcal{F}(P, Q)$ en $p$ si $\Lambda-\{p\}$ est contenu dans une feuille $\overline{\mathrm{de}} \mathcal{F}(P, Q)$.

Lemme II.3.10. Soit $\Lambda$ une séparatrice locale de $\mathcal{F}(P, Q)$ en $p \in X$. Supposons que:

i) $f^{\prime}(p)=a \in \mathbb{C}$; ii) $\left.f^{\prime}\right|_{\Lambda}$ n'est pas constante; iii) $\Lambda-\{p\} \subset U$. Alors $g^{\prime}(\Lambda)$ est le support d'une solution locale en a de (II.1) et cette solution est ramifiée si le degré de l'application locale $f^{\prime}:(\Lambda, p) \rightarrow(\mathbb{C}, a)$ est plus grand que 1 .

Démonstration. Le germe $\pi(\Lambda)$ admet une paramétrisation :

$$
x=\alpha(t), y=\beta(t), z=\gamma(t)
$$

où $\alpha$ est holomorphe et non-constante et $\beta, \gamma$ sont méromorphes au voisinage de $0 \in \mathbb{C}, \alpha(0)=a$ et

$$
F(\alpha(t), \beta(t), \gamma(t))=0 \text { si } t \neq 0 .
$$

Par le lemme II.3.3, $\left.\pi^{*}(\omega)\right|_{U}$ est holomorphe et jamais nulle. Alors, par hypothèse :

$$
\left.\pi^{*}(\omega)\right|_{\Lambda-\{p\}}=0
$$

Donc

$$
\left.\omega\right|_{\pi(\Lambda)-\{\pi(p)\}}=0 .
$$

Il en résulte :

$$
P(\alpha(t), \beta(t), \gamma(t)) \alpha^{\prime}(t)-Q(\alpha(t), \beta(t), \gamma(t)) \beta^{\prime}(t)=0 \text { si } t \neq 0 .
$$

On en déduit tout de suite que $x=\alpha(t), y=\beta(t)$ est une solution locale en $a$ dont le support est $g(\pi(\Lambda))=g^{\prime}(\Lambda)$.

La dernière affirmation vient du fait que $\pi(\Lambda)$ est un relèvement du support de la solution locale considérée et de II.3.2.

Lemme II.3.11. Soit $\Sigma$ le support d'une solution locale par $q:=(a, b) \in$ $\mathbb{C} \times \mathbb{P}_{1}$ de (II.1). Supposons que $\Sigma-\{q\} \subset \mathbb{C}^{2}-\Delta$. Alors, il existe un point $p \in X$ et une séparatrice locale $\Lambda$ de $\mathcal{F}(P, Q)$ en $p$ tels que $g^{\prime}(p)=q$ et $g^{\prime}(\Lambda)=\Sigma$. Si la solution est ramifiée on peut choisir $\Lambda$ de telle façon que $f^{\prime}:(\Lambda, p) \rightarrow(\mathbb{C}, a)$ soit de degré $>1$. 
Démonstration. Soit $x=\alpha(t), y=\beta(t)$ la solution dont $\Sigma$ est le support. D'après le lemme II.1.3 on peut supposer qu'il existe $z=\gamma(t)$ telle que

$$
t \mapsto(\alpha(t), \beta(t), \gamma(t))
$$

est un germe $L$ de courbe analytique irréductible en $p^{\prime}=(\alpha(0), \beta(0), \gamma(0)) \in$ $S$ avec

$$
L-\left\{p^{\prime}\right\} \subset V \text { et }\left.\omega\right|_{L-\left\{p^{\prime}\right\}}=0 .
$$

D'après le lemme II.3.3a :

$$
\pi^{-1}\left(L-\left\{p^{\prime}\right\}\right)=\Lambda-\{p\}
$$

où $\Lambda$ est un germe de courbe analytique irréductible en $p \in X$ avec $\pi(p)=p^{\prime}$ ([15, chap. K, thm. 7]). Alors,

$$
\left.\omega\right|_{\left(L-\left\{p^{\prime}\right\}\right)}=0 \text { implique }\left.\pi^{*}(\omega)\right|_{\Lambda-\{p\}}=0 .
$$

D'après le lemme II.3.3, cela entraîne que $\Lambda$ est une séparatrice locale de $\mathcal{F}(P, Q)$.

La dernière assertion suit de II.3.2.

Lemme II.3.12. Soit $C_{0} \not \subset \Delta$ une solution algébrique de (II.1). Alors, il existe une courbe algébrique projective $E \subset X$ invariante par $\mathcal{F}(P, Q)$ telle que $g^{\prime}(E)$ est l'adhérence $C$ de $C_{0}$ dans $\mathbb{P}_{1}^{2}$.

Démonstration. $\left(g^{\prime}\right)^{-1}(C) \subset X$ est une courbe algébrique projective. D'après le lemme II.3.11 cette courbe contient une composante irréductible $E$ invariante par $\mathcal{F}(P, Q)$ telle que $\left.g^{\prime}\right|_{E}$ n'est pas constante (voir annexe, lemme A.4.3).

Définition II.3.13. Un feuilletage analytique à singularités isolées $\mathcal{F}$ de $X$ est génériquement transverse à $h$ si $\mathcal{F}$ est transverse à au moins une fibre régulière de $h$.

Dans les cas où $h$ est une fibration rationnelle (elliptique) un tel feuilletage est aussi appelé de Riccati (resp. tourbillonné ou turbulent) (voir [5] et [6]) Dès qu'il n'ait pas d'ambiguité par rapport à la surface fibrée considérée, on dira tout simplement que le feuilletage en question est génériquement transverse.

Proposition II.3.14. Si $\mathcal{F}$ est génériquement transverse, il existe un ensemble fini $E \subset B$ tel que :

a) pour tout $b \in B-E, h^{-1}(b)$ est une fibre régulière transverse à $\mathcal{F}$;

b) les fibres qui ne sont pas transverses à $\mathcal{F}$ contiennent des composantes irréductibles invariantes par $\mathcal{F}$. 
Démonstration. En effet, si $\mathcal{F}$ est génériquement transverse, le support du diviseur de tangence entre $\mathcal{F}$ et la fibration $h$ est contenu dans un nombre fini de fibres de $h([5, \S 1]$ et annexe $\S 5)$.

Théorème II.3.15. Les conditions suivantes sont équivalentes :

a) L'équation (II.1) n'est pas dans la classe FP.

b) $\mathcal{F}(P, Q)$ n'est pas génériquement transverse.

c) Tout $a \in \mathbb{C}$ générique est point de ramification de (II.1).

En particulier, l'équation (II.1) est dans la classe FP si et seulement si $\mathcal{F}(P, Q)$ est génériquement transverse.

Démonstration. $(\mathrm{b}) \Rightarrow(\mathrm{c})$. Supposons que $\mathcal{F}(P, Q)$ ne soit pas génériquement transverse. Pour $s \in B$ générique on peut supposer que :

i) $X_{s}=h^{-1}(s)$ est une fibre régulière et $a=\varphi(s) \in \mathbb{C}$;

ii) $X_{s}$ n'est pas invariant par $\mathcal{F}(P, Q)$ et ne contient pas de point singulier de $\mathcal{F}(P, Q)$;

iii) $X_{s}$ n'est pas contenue dans la courbe $T:=X-U$;

iv) chaque point de $X_{s} \cap T$ est non-singulier dans $T$;

v) $d\left(\left.h\right|_{T}\right)(u) \neq 0$ pour tout $u \in X_{s} \cap T$.

Il existe $p \in X_{s}$ tel que le germe $\Lambda$ de courbe analytique irréductible en $p$ invariant par $\mathcal{F}(P, Q)$ est tangent à $X_{s}$ et $\Lambda \not \subset X_{s}$. De plus $\Lambda$ n'est pas contenu dans $T$, car sinon il serait transverse à $X_{s}$. Alors, $\Lambda-\{p\} \subset U$. D'après le lemme II.3.10 il existe une solution locale en $a$ ramifiée.

(a) $\Rightarrow$ (b) Supposons que l'équation ne soit pas dans la classe FP. Si $C_{0} \subset \mathbb{C}^{2}$ est une courbe algébrique, alors pour tout $a \in \mathbb{C}$ générique, toute solution locale en $a$ dont le support est contenu dans l'adhérence $C$ de $C_{0}$ dans $\mathbb{P}_{1}^{2}$ est non-ramifiée. En effet, tout relèvement du support d'une telle solution est contenu dans $g^{-1}(C)$. Or, $g^{-1}(C) \subset S$ est une courbe algébrique : il suffit de prendre $a$ en dehors des images par $f$ des points singuliers et des points à tangente verticale (par rapport à $f$ ) de cette courbe (voir lemme II.3.2).

Comme l'équation n'est pas dans la classe FP, il s'ensuit qu'il existe une infinité de points $a \in \mathbb{C}$ tels que par un $q=(a, b)\left(b \in \mathbb{P}_{1}\right)$ il passe une solution locale ramifiée $\Sigma$ telle que :

$$
\Sigma-\{q\} \subset \mathbb{C}-\Delta .
$$

Par les lemmes II.3.11 et II.3.10 il existe une séparatrice locale $\Lambda$ de $\mathcal{F}(P, Q)$ en $p \in X$ telle que $f^{\prime}(p)=a$ et $f^{\prime}:(\Lambda, p) \rightarrow(\mathbb{C}, a)$ est finie et de degré $>1$. Alors $\mathcal{F}(P, Q)$ n'est pas transverse à la fibre de $h$ par $p$. Donc, $\mathcal{F}(P, Q)$ n'est pas génériquement transverse.

(c) $\Rightarrow$ (a) Se déduit de la définition.

Exemple II.3.16. Supposons qu'il existe des fonctions rationnelles

$$
y=A(x, t), z=B(x, t)
$$


telles que, pour $a \in \mathbb{C}$ générique,

$$
y=A(a, t), z=B(a, t)
$$

est une paramétrisation de la courbe $F(a, y, z)=0$ (tout point générique de la courbe correspond à une seule valeur du paramètre).

Par ce changement de variable dépendante l'équation (II.1) se transforme en une équation explicite de la forme

$$
\frac{d t}{d x}=H(x, t)
$$

Si l'on suppose, en plus, que (II.1) est dans la classe FP, alors (II.4) est une équation de Riccati; c'est-à-dire

$$
H(x, t)=a(x) t^{2}+b(x) t+c(x)
$$

où $a, b, c$ sont des fonctions rationnelles. En effet, soit $p_{1}: \mathbb{P}_{1}^{2} \rightarrow \mathbb{P}_{1}$ la projection sur le premier facteur. Alors,

$$
x=x, y=A(x, t), z=B(x, t)
$$

définit une application birationnelle $\theta: \mathbb{P}_{1}^{2} \rightarrow S$ telle que $f \circ \theta=p_{1}$.

Dans cet exemple, $F(a, y, z)=0$ est une courbe irréductible de genre 0 pour $a \in \mathbb{C}$ générique; alors, dans les notations du début de ce paragraphe, $B=\mathbb{P}_{1}, \varphi=I d, f^{\prime}=h$ et les fibres régulières de $h$ sont isomorphes à $\mathbb{P}_{1}$. On en déduit que $\mu:=\pi^{-1} \circ \theta$ est une application birationnelle $\mathbb{P}_{1}^{2} \rightarrow X$ telle que $h \circ \mu=p_{1}$.

L'équation (II.4) définit un feuilletage de $\mathbb{P}_{1}^{2}$ qui correspond avec $\mathcal{F}(P, Q)$ par $\mu$. D'après le théorème II.3.15 $\mathcal{F}(P, Q)$ est génériquement transverse. Comme les courbes qui se contractent par $\mu$ ne peuvent être que des composantes de fibres de $p_{1}$, on voit que ce feuilletage de $\mathbb{P}_{1}^{2}$ est génériquement transverse à $p_{1}$ dans le sens de la définition II.3.13. Cela implique que l'équation (II.4) est de Riccati ([18, thm. 3.3.3]).

Par exemple, pour obtenir l'intégrale première de l'exemple I.5.4 on fait le changement :

$$
y=A(x, t):=-\frac{t}{x^{2} t^{2}-2 x t+1}, z=B(x, t)=-\frac{t^{2}}{x^{2} t^{2}-2 x t+1},
$$

et on intègre l'équation

$$
\frac{d t}{d x}=-t^{2}
$$

ainsi obtenue.

Observation II.3.17. Supposons que l'équation $F\left(x, y, y^{\prime}\right)=0$ soit dans la classe FP. Soit $p:=(a, b, c)$ un point non-singulier de $S$. Comme $\pi^{-1}$ 
est un isomorphisme au voisinage de $p$ qui commute avec $f$ et $f^{\prime}$, on a que $\left(\left.d x\right|_{S}\right)(p) \neq 0$ pour $a$ générique. Pour la même raison et d'après la définition II.3.13, la proposition II.3.14 et le théorème II.3.15, si $\left(\left.\omega\right|_{S}\right)(p) \neq 0$ alors $\left(\left.d x\right|_{S}\right)(p) \wedge\left(\left.\omega\right|_{S}\right)(p) \neq 0$ pour a générique. Donc,

$$
(d x \wedge \omega \wedge d F)(p)=0 \text { implique }(\omega \wedge d F)(p)=0
$$

pour $a$ générique, car $\omega=z d x-d y$ dans ce cas.

Il est clair que si $p$ est un point singulier de $S$ alors

$$
c F_{y}(a, b, c)+F_{x}(a, b, c)=0 .
$$

En conclusion,

$$
F(a, b, c)=F_{z}(a, b, c)=0 \text { implique } c F_{y}(a, b, c)+F_{x}(a, b, c)=0
$$

pour a générique. De ceci et de la Nullstellensatz on déduit qu'il existe $m \in \mathbb{N}$ tel que

$$
\left(z F_{y}(x, y, z)+F_{x}(x, y, z)\right)^{m}=A(x, y, z) F(x, y, z)+B(x, y, z) F_{z}(x, y, z)
$$

où $A, B$ sont des polynômes en $y, z$ à coefficients fonctions rationnelles de $x$. Cela remplace la condition nécessaire 4 de [29] qui est fausse comme le montre l'équation $\left(y^{\prime}\right)^{3}-x y^{2}=0$.

Dans la suite on donnera quelques applications aux intégrales premières et aux solutions algébriques.

Proposition II.3.18. Soit $R(x, y, \lambda)$ une intégrale première rationnelle de (II.1). Alors, il existe un ouvert de Zariski non-vide $W$ de $\mathbb{C}^{2}$ tel que:

a) toute solution par $q \in W$ est régulière;

b) si $y=y(x)$ est une solution par $q \in W$, il existe $\lambda_{0} \in \mathbb{C}$ tel que $R\left(x, y(x), \lambda_{0}\right)=0$.

Démonstration. En considérant l'équation polynomiale associée (définition II.2.1) on voit qu'il suffit de considérer le cas de l'équation $F\left(x, y, y^{\prime}\right)=0$ (proposition II.2.2 et corollaire II.2.5). Soit $W \subset \mathbb{C}^{2}$ l'ouvert de Zariski défini par :

$$
c_{0}(x, y) D(x, y) \neq 0 .
$$

Soient $S_{0}$ et $g_{0}: S_{0} \rightarrow \mathbb{C}^{2}$ définis comme au début de ce paragraphe et soit $V_{1}:=g_{0}^{-1}(W)$. Alors $g_{0}: V_{1} \rightarrow W$ est un revêtement fini connexe non-ramifié.

Notons $T \subset \mathbb{C}^{3}$ la surface définie par $R(x, y, \lambda)=0$ et $h: T \rightarrow \mathbb{C}^{2}$, l'application définie par $h(x, y, \lambda)=(x, y)$. En prenant $W$ éventuellement plus petit on peut supposer d'une part que $h: V_{2} \rightarrow W$ est aussi un revêtement fini connexe non-ramifié, où $V_{2}=h^{-1}(W)$; d'autre part que $R_{y}$ ne s'annule jamais sur $V_{2}$. 
Soit $\varphi: V_{2} \rightarrow \mathbb{C}^{3}$ définie par

$$
\varphi(x, y, \lambda)=\left(x, y,-R(x, y, \lambda) / R_{y}(x, y, \lambda)\right) .
$$

Par le corollaire I.5.5, $\varphi\left(V_{2}\right) \subset S_{0}$. Alors, comme $g_{0} \circ \varphi=h$, on a que $\varphi\left(V_{2}\right) \subset V_{1}$ et que $\varphi: V_{2} \rightarrow V_{1}$ est un isomorphisme local, propre et ouvert; en particulier, il est surjectif.

Soit $q \in W$. L'affirmation a) découle du corollaire I.1.4

Soit $y=y(x)$ une solution régulière par $q$. Si $q=(a, b)$ et $c=y^{\prime}(a)$ on a :

$$
(a, b, c) \in V_{1} \text {. }
$$

De ce qui précède il résulte qu'il existe $\lambda(x)$ holomorphe au voisinage de a telle que :

$$
(x, y(x), \lambda(x)) \in V_{2} \text { et } \varphi(x, y(x), \lambda(x))=\left(x, y(x), y^{\prime}(x)\right) .
$$

Alors

$$
R(x, y(x), \lambda(x))=0 \text { et } y^{\prime}(x)=-\frac{R_{x}(x, y(x), \lambda(x))}{R_{y}(x, y(x), \lambda(x))} .
$$

En dérivant la première égalité et en utilisant la deuxième on obtient :

$$
R_{\lambda}(x, y(x), \lambda(x)) \lambda^{\prime}(x)=0 .
$$

Comme $h$ est un isomorphisme local, $R_{\lambda}$ ne s'annule jamais sur $V_{2}$. Alors $\lambda^{\prime}(x)=0$. Donc, $\lambda(x)=$ cte. $=\lambda_{0} \in \mathbb{C}$

Théorème II.3.19. Il existe une infinité de solutions algébriques si et seulement s'il existe une intégrale première rationnelle.

Démonstration. Si $R(x, y, \lambda)$ est une intégrale première alors, pour $\lambda_{0} \in \mathbb{C}$ générique, $R\left(x, y, \lambda_{0}\right)=0$ définit une courbe algébrique dont les composantes irréductibles sont des solutions algébriques.

Réciproquement, supposons qu'il existe une infinité de solutions algébriques. Il en résulte, d'après le lemme II.3.12, qu'il existe une infinité de courbes algébriques irréductibles invariantes par $\mathcal{F}(P, Q)$. Par $[21], \mathcal{F}(P, Q)$ admet une intégrale première rationnelle. La preuve du théorème suit, alors, du lemme suivant.

Lemme II.3.20. Si le feuilletage $\mathcal{F}(P, Q)$ possède une intégrale première rationnelle, alors il existe une intégrale première rationnelle de l'équation (II.1). 
Démonstration. Soit $\Theta$ une intégrale première rationnelle de $\mathcal{F}(P, Q)$. Posons $\Gamma:=\Theta \circ \pi^{-1}$. C'est une fonction rationnelle non-constante sur $S_{0}$. On peut représenter $\Gamma$ par un quotient

$$
\frac{A(x, y, z)}{B(x, y, z)}, \quad A, B \in \mathbb{C}[x, y, z]
$$

où $A, B$ sont premiers entre eux et $F$ ne divise pas $B$. Le sous-ensemble algébrique de $\mathbb{C}^{3}$ définit par :

$$
F(x, y, z)=B(x, y, z)=0
$$

est de dimension $\leq 1$. Alors, il existe $L(x, y) \in \mathbb{C}[x, y]$ non-nul qui s'annule sur cet ensemble. Notons :

$$
W=\left\{(x, y, z) \in S_{0}: c_{0}(x, y) L(x, y) \neq 0,(x, y) \notin \Delta\right\} ;
$$

l'ensemble $W$ est une variété analytique complexe non-vide de dimension 2, connexe.

Par ailleurs, notons $Y \subset \mathbb{C}^{4}$ l'ensemble algébrique de dimension pure 2 défini par :

$$
A(x, y, z)-\lambda B(x, y, z)=F(x, y, z)=0 .
$$

Considérons maintenant l'application analytique $\varphi: W \rightarrow \mathbb{C}^{4}$ définie par :

$$
\varphi(x, y, z)=\left(x, y, z, \frac{A(x, y, z)}{B(x, y, z)}\right) .
$$

Par le critère jacobien on a que $\varphi(W) \subset Y-\operatorname{Sing} Y$. Comme $W$ est connexe, $\varphi(W)$ est contenu dans une composante irréductible $Y^{\prime}$ de $Y$. Puisque $\operatorname{dim} Y^{\prime}=2$, il existe $R(x, y, \lambda) \in \mathbb{C}[x, y, \lambda]$ irréductible qui s'annule sur $Y^{\prime}$. C'est-à-dire,

$$
R(x, y, A(x, y, z) / B(x, y, z))=0 \text { si }(x, y, z) \in W .
$$

Il est évident que $R$ dépend effectivement de $\lambda$ et de $x$ ou $y$, puisque $\Gamma$ n'est pas constante.

Soient $a, b \in \mathbb{C}$ tels que :

$$
c_{0}(a, b) L(a, b) \neq 0 \text { et }(a, b) \notin \Delta .
$$

Soit $x=\alpha(t), y=\beta(t)$ une solution de (II.1) par $(a, b)$. Par le lemme II.1.3 on peut supposer qu'il existe $z=\gamma(t)$ méromorphe au voisinage de $0 \in \mathbb{C}$ telle que

$$
P(\alpha(t), \beta(t), \gamma(t)) \alpha^{\prime}(t)-Q(\alpha(t), \beta(t), \gamma(t)) \beta^{\prime}(t)=F(\alpha(t), \beta(t), \gamma(t))=0 .
$$

Comme $c_{0}(a, b) \neq 0, \gamma(0) \in \mathbb{C}$. Il en résulte que le germe de courbe :

$$
t \mapsto(\alpha(t), \beta(t), \gamma(t)),
$$


est contenu dans $W$ et satisfait à l'équation $\omega=0$. De plus, comme $W$ est non-singulière, la restriction de $\pi$ à $\pi^{-1}(W)$ est un isomorphisme sur $W$ (voir diagramme du début de ce paragraphe). Alors, d'après le lemme II.3.3b, la courbe

$$
t \mapsto \pi^{-1}(\alpha(t), \beta(t), \gamma(t))
$$

est invariante par $\mathcal{F}(P, Q)$, car $W \subset V$; elle est contenue dans l'ouvert où $\Theta$ est holomorphe, d'après la définition de $W$. Donc,

$$
\Theta\left(\pi^{-1}(\alpha(t), \beta(t), \gamma(t))\right)=\text { cte. },
$$

d'où suit qu'il existe $\lambda_{0} \in \mathbb{C}$ tel que

$$
\frac{A(\alpha(t), \beta(t), \gamma(t))}{B(\alpha(t), \beta(t), \gamma(t))}=\lambda_{0} \text {. }
$$

On en déduit que

$$
\begin{aligned}
0 & =R\left(\alpha(t), \beta(t), \frac{A(\alpha(t), \beta(t), \gamma(t))}{B(\alpha(t), \beta(t), \gamma(t))}\right) \\
& =R\left(\alpha(t), \beta(t), \lambda_{0}\right) .
\end{aligned}
$$

Il en résulte que $R$ dépend effectivement de $y$ et, par la proposition II.2.6, que $R$ est une intégrale première de (II.1).

Corollaire II.3.21. Si $C_{1}, C_{2}, C_{3}, \ldots$ est une suite de solutions algébriques de (II.1) toutes différentes, alors le genre de $C_{n}$ ne dépend pas de $n$ pour tout $n$ assez grand.

Démonstration. Par le théorème II.3.19, il existe une intégrale première rationnelle $R(x, y, \lambda)$ de (II.1). Soit $W$ l'ouvert de la proposition II.3.18. Alors, $C_{n} \cap W$ n'est pas vide si $n$ est assez grand. Supposons que $y=y_{n}(x)$ soit l'équation locale de $C_{n}$ au voisinage d'un point $q \in C_{n} \cap W$. Alors, il existe $\lambda_{n} \in \mathbb{C}$ tel que $R\left(x, y_{n}(x), \lambda_{n}\right)=0$. Alors $C_{n}$ est une composante irréductible de la courbe $D_{n}$ définie par $R\left(x, y, \lambda_{n}\right)=0$. Comme les $C_{j}$ sont toutes différentes, on peut supposer que, pour $n$ assez grand, $\lambda_{n}$ est générique dans le sens de la proposition II.3.5 appliquée à $R$. Alors, les composantes irréductibles de $D_{n}$ ont toutes le même genre qui ne dépend pas de $n$.

Exemple II.3.22. Considérons l'équation différentielle

$$
\left(y^{\prime}\right)^{2}-2 y y^{\prime}+y^{2}-y=0 .
$$

On vérifie, par les théorèmes I.4.11 et I.4.13 et le lemme I.2.6, que l'équation est dans la classe FP. Son genre est 0. Comme pour l'exemple II.3.16 on prend

$$
y=\frac{1}{(t-1)^{2}}, z=\frac{t}{(t-1)^{2}}
$$


et on trouve l'équation de Riccati

$$
-2 \frac{d t}{d x}=t^{2}-t
$$

On la résout par le changement de variable $u=1 / t$ et on obtient la solution générale :

$$
y=\frac{\left(1+c e^{-x / 2}\right)^{2}}{c^{2} e^{-x}}, y=0, y=1 .
$$

On voit tout de suite que cette équation n'admet pas d'intégrale première rationnelle; elle possède la solution algébrique $y=1$, qui n'est pas contenue dans $\Delta$.

\section{II.4 Solutions méromorphes}

Considérons l'équation de Riccati

$$
y^{\prime}=p(x) y^{2}+q(x) y+r(x),
$$

avec $p, q, r \in \mathbb{C}(x)$ (comparer avec le lemme I.4.5).

Notons $S \subset \mathbb{C}$ l'ensemble des pôles de $p, q$ et $r$. Soient $a \in \mathbb{C}-S$ et $y=y(x)$ une solution locale uniforme de cette équation en $a$. Soit $U$ un ouvert simplement connexe tel que $a \in U \subset \mathbb{C}-S$. L'une des propriétés remarquables des équations de Riccati (voir $[18, \S 3.2$ et $\S 3.3]$ ) nous dit que $y=y(x)$ se prolonge à une solution méromorphe dans $U$ tout entier. Dans ce paragraphe nous généralisons cette propriété aux équations différentielles comme dans (II.1) qui sont dans la classe FP.

Définition II.4.1. Soit $f \in \mathbb{C}(U)$ avec $U \subset \mathbb{C}$ un ouvert connexe. Alors $y=f(x)$ est une solution méromorphe de (II.1) dans $U$ si elle détermine une solution uniforme locale de (II.1) en chaque point $a \in U$. Dans le cas où $f$ est holomorphe on dira que la solution est holomorphe

Lemme II.4.2. Soit $f \in \mathbb{C}(U)$ avec $U \subset \mathbb{C}$ un ouvert connexe. Alors $y=f(x)$ est une solution méromorphe de (II.1) dans $U$ si et seulement s'il existe un sous-ensemble discret $S$ de $U$, contenant les pôles de $f$, tel que pour tout $x \in U-S$ les équations en $z$ suivantes :

$$
P(x, f(x), z)-Q(x, f(x), z) f^{\prime}(x)=0, F(x, f(x), z)=0,
$$

ont une racine en commun.

Démonstration. Elle résulte immédiatement des définitions du $§ 1$.

Exemple II.4.3. La fonction $f(x)=x / 2$ est une solution holomorphe dans $\mathbb{C}$ de l'équation

$$
d x-x z d y=0,(y-x) z+1=0
$$

avec $S=\{0\}$ non-vide. 
Proposition II.4.4. Soit $f \in \mathbb{C}(U)$ avec $U \subset \mathbb{C}$ un ouvert connexe. Supposons qu'il existe $a \in U$ tel que $y=f(x)$ détermine une solution locale uniforme de (II.1) en a. Alors, $y=f(x)$ est une solution méromorphe de (II.1) dans $U$.

Démonstration. Considérons le sous-ensemble algébrique $S$ de $\mathbb{C}^{5}$ défini par

$$
P(x, y, z) u-Q(x, y, z) v=F(x, y, z)=0 ;
$$

notons $T \subset \mathbb{C}^{4}$ l'image de $S$ par la projection $(x, y, z, u, v) \mapsto(x, y, u, v)$.

Par ailleurs, considérons l'application analytique injective $\phi: U \rightarrow \mathbb{P}_{1}^{4}$ définie par

$$
\phi(x)=\left(x, f(x), 1, f^{\prime}(x)\right) .
$$

D'après le lemme II.4.2, il suffit de démontrer que $U-\phi^{-1}(T)$ est un sous-ensemble discret et fermé de $U$.

Puisque $T$ est un sous-ensemble constructible de $\mathbb{P}_{1}^{4}$ ([16, thm. 3.16]), l'un de sous-ensembles $U-\phi^{-1}(T)$ ou $\phi^{-1}(T)$ doit être discret et fermé dans $U$. Or, par hypothèse, il existe un voisinage $V_{a} \subset U$ de $a$ tel que $\phi\left(V_{a}-\{a\}\right) \subset T$, et donc $\phi^{-1}(T)$ n'est pas discret d'où l'assertion.

Théorème II.4.5. Supposons que l'équation (II.1) soit dans la classe FP. Il existe un ensemble fini $S \subset \mathbb{C}$ tel que si $\Omega \subset \mathbb{C}-S$ est un ouvert simplement connexe et $a \in \Omega$, alors toute solution locale de (II.1) en a est uniforme et se prolonge à une solution méromorphe de (II.1) dans $\Omega$.

Démonstration. On reprend les notations et définitions du début du $\S 3$.

Soit $\Gamma$ l'adhérence dans $\mathbb{C} \times \mathbb{P}_{1}$ de $\Delta$ (voir $\S 1$ ).

Soit $S$ un sous-ensemble fini de $\mathbb{C}$ tel que :

i) si $a \in \mathbb{C}-S$, toute solution locale de (II.1) en $a$ est uniforme;

ii) Si $a \in \mathbb{C}-S$ et $q=(a, b) \in \Gamma$, alors $q$ est un point régulier de $\Gamma$ à tangente non-verticale;

iii) tout $a \in \mathbb{C}-S$ est une valeur régulière de $\varphi$ (voir diagramme du début du $\S 3)$;

iv) si $a \in \mathbb{C}-S$, tout $\xi \in \varphi^{-1}(a)$ est une valeur régulière de $h$ et $h^{-1}(\xi)$ est transverse à $\mathcal{F}(P, Q)$ (voir théorème II.3.15 et définition II.3.13).

Notons $\Omega^{\prime}:=\mathbb{C}-S$. Prenons $a \in \Omega^{\prime}$ et soit $y=y(x)$ une solution locale de (II.1) en $a$. Posons $q=(a, b)$ avec $b:=y(a) \in \mathbb{P}_{1}$ et notons $\Sigma$ le support de cette solution. On distingue deux cas :

Premier cas : $\Sigma-\{q\} \subset \mathbb{C}^{2}-\Delta$. Soit $\mathcal{F}$ la restriction de $\mathcal{F}(P, Q)$ à $h^{-1}\left(\varphi^{-1}\left(\Omega^{\prime}\right)\right)$; de iv) suit que $\mathcal{F}$ est régulier. D'après le lemme II.3.11, il existe une séparatrice locale $\Lambda$ de $\mathcal{F}$ telle que $g^{\prime}(\Lambda)=\Sigma$; en particulier $\Lambda$ est contenue dans une feuille de $\mathcal{F}$. Puisque les feuilles de $\mathcal{F}$ sont transverses aux fibres de $h$ au-dessus de $\varphi^{-1}\left(\Omega^{\prime}\right)$, il existe une surface de Riemann $L$ et une application analytique $\alpha: L \rightarrow X$ telles que $h \circ \alpha: L \rightarrow \varphi^{-1}\left(\Omega^{\prime}\right)$ est 
un revêtement non-ramifié et $\Lambda \subset \alpha(L)$. Alors, $g^{\prime}(\alpha(L)) \supset \Sigma$ (voir annexe, définition A.4.1 et corollaire A.6.4).

On a le diagramme commutatif suivant

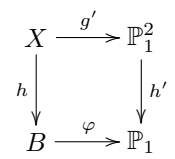

où $h^{\prime}$ est la projection sur le premier facteur. De ceci et du fait que la restriction de $\varphi$ à $\varphi^{-1}\left(\Omega^{\prime}\right)$ est un revêtement fini non-ramifié, on déduit que l'application

$$
h^{\prime} \circ g^{\prime} \circ \alpha: L \longrightarrow \Omega^{\prime}
$$

l'est aussi.

Prenons maintenant un ouvert simplement connexe $\Omega$ tel que $a \in \Omega \subset \Omega^{\prime}$. Il existe un ouvert $W \subset L$ tel que $\Lambda \subset \alpha(W)$ et $h^{\prime} \circ g^{\prime} \circ \alpha$ applique isomorphiquement $W$ sur $\Omega$. Alors $g^{\prime}(\alpha(W)) \subset \Omega \times \mathbb{P}_{1}$ est le graphe d'une fonction méromorphe sur $\Omega$ qui prolonge $y=y(x)$. On conclut par la proposition II.4.4.

Deuxième cas : $\Sigma \subset \Gamma$. Soit $K$ la composante irréductible de $\Gamma$ qui contient

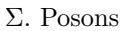

$$
K_{0}:=\left\{(x, y) \in K: x \in \Omega^{\prime}\right\} .
$$

Alors $K_{0}$ est une surface de Riemann ([15, vol. II(E), thm. 19]) contenant $\Sigma$ telle que la restriction de $h^{\prime}$ à $K_{0}$ induit un isomorphisme local propre sur $\Omega^{\prime}$; en particulier $h^{\prime}$ est un revêtement fini non-ramifié. On complète la preuve comme dans le premier cas.

\section{II.5 Singularités essentielles}

Un fait remarquable pour l'équation (II.1) est que sa solution générale ne possède pas de "singularité essentielle mobile" (comparer avec [20, 3.16] et [18, thm.3.3.2]). Plus précisément, on a la définition et le théorème suivants.

Définition II.5.1. Soit $a \in \mathbb{C}$. Alors a est une singularité essentielle de (II.1) s'il existe un disque ouvert $D \subset \mathbb{C}$ de centre 0 et des fonctions

$$
\alpha: D \rightarrow \mathbb{C}, \beta: D^{*} \rightarrow \mathbb{C}\left(D^{*}:=D-\{0\}\right)
$$

telles que $\alpha(0)=a$, $\alpha$ est holomorphe et non-constante, $\beta$ est méromorphe, 0 est une singularité essentielle de $\beta$ et les équations en $z$

$$
P(\alpha(t), \beta(t), z) \alpha^{\prime}(t)-Q(\alpha(t), \beta(t), z) \beta^{\prime}(t)=0, F(\alpha(t), \beta(t), z)=0
$$

ont une racine en commun en tout $t \in D^{*}$ qui ne soit pas un pôle de $\beta$. 
Théorème II.5.2. Il n'existe qu'un nombre fini de singularités essentielles de l'équation (II.1).

La preuve de ce théorème résultera du lemme II.5.4 ci-dessous.

Lemme II.5.3. Soit $\mathcal{F}$ un feuilletage analytique à singularités isolées de la surface analytique non-singulière $X$. Soit $M \subset X$ un sous-ensemble analytique, compact, de dimension 1 . Supposons que $p_{1}, p_{2}, \ldots, p_{n}$ soient des points non-singuliers de $M$ tels que $\mathcal{F}$ et $M$ sont transverses en chaque $p_{j}(j=1, \ldots, n)$. Alors, il existe un ouvert $U$ de $X$ contenant $M$ tel que si $F$ est une feuille de $\left.\mathcal{F}\right|_{(U-M)}$ dont l'adhérence $\bar{F}$ dans $U$ contient un point $p_{i}$, alors $\bar{F} \cap M=\left\{p_{i}\right\}$.

Démonstration. La structure produit de $\mathcal{F}$ au voisinage de chaque $p_{j}$ (voir annexe, proposition A.1.7) implique que, pour $U$ convenable, une telle feuille $F$ doit être un germe de courbe analytique transverse à $M$ au point $p_{i}$, moins le point $p_{i}$.

Pour le lemme qui suit on garde les notations des $\S 1$ et $\S 3$.

Lemme II.5.4. Soit $a \in \mathbb{C}$ tel que $c_{0}(a, y) \neq 0$ et que la droite $x=a$ ne soit pas contenue dans $\Delta$. Supposons que a est une singularité essentielle de (II.1). Alors, l'une (au moins) des composantes irréductibles de $\left(f^{\prime}\right)^{-1}(a)$ est invariante par $\mathcal{F}(P, Q)$.

Démonstration. Supposons, par l'absurde, qu'aucune des composantes irréductibles de $\left(f^{\prime}\right)^{-1}(a)$ ne soit invariante par $\mathcal{F}(P, Q)$; notons $D$ le diviseur de tangence entre le feuilletage défini par $f^{\prime}$ et $\mathcal{F}(P, Q)$. Alors, l'intersection de $\left(f^{\prime}\right)^{-1}(a)$ avec le support de $D$ est finie (voir annexe, exemple A.2.4, définition A.5.1 et proposition A.5.2). Cela implique qu'il existe $b \in \mathbb{C}$ tel que $c_{0}(a, b) \neq 0,(a, b) \notin \Delta$ et que $\left(g^{\prime}\right)^{-1}(a, b)$ est disjoint du support de $D$ (voir début du $\S 3$ ).

Soit $q:=(a, b)$. Des deux premières conditions il résulte que $g^{-1}(q)$ est fini et contenu dans $V$. Alors, $\left(g^{\prime}\right)^{-1}(q)$ est fini. De la troisième on déduit que si $p \in\left(g^{\prime}\right)^{-1}(q)$ alors $p$ est un point non-singulier de $\left(f^{\prime}\right)^{-1}(a)$ et de $\mathcal{F}(P, Q)$; de plus $\left(f^{\prime}\right)^{-1}(a)$ et $\mathcal{F}(P, Q)$ sont transverses en $p$ (voir annexe, proposition A.5.2).

D'après le lemme II.5.3, il existe un voisinage ouvert $V_{a}$ de $a$ tel que si $F$ est une feuille de

$$
\left.\mathcal{F}(P, Q)\right|_{\left(f^{\prime}\right)^{-1}\left(V_{a}^{*}\right)} \quad\left(V_{a}^{*}:=V_{a}-\{a\}\right)
$$

et si l'adhérence $\bar{F}$ de $F$ dans $\left(f^{\prime}\right)^{-1}\left(V_{a}\right)$ contient un point $p \in\left(g^{\prime}\right)^{-1}(q)$, alors $\bar{F} \cap\left(f^{\prime}\right)^{-1}(a)=\{p\}$.

Soient $x=\alpha(t), y=\beta(t)$ comme dans la définition II.5.1. On peut supposer que $\alpha(D)=V_{a}, \alpha^{-1}(a)=0$ et que le morphisme $\alpha: D \rightarrow V_{a}$ 
est propre; en particulier $\left.\alpha\right|_{D^{*}}: D^{*} \rightarrow V_{a}^{*}$ est propre. Donc, l'application analytique

$$
\Gamma: D^{*} \rightarrow V_{a}^{*} \times \mathbb{P}_{1} \subset \mathbb{P}_{1}^{2}, \Gamma(t)=(\alpha(t), \beta(t)), t \in D^{*}
$$

est aussi propre. Alors $Y=\Gamma\left(D^{*}\right)$ est un sous-ensemble analytique irréductible de dimension 1 de $V_{a}^{*} \times \mathbb{P}_{1}$. Comme 0 est une singularité essentielle de $\beta$, l'adhérence $\bar{Y}$ de $Y$ dans $V_{a} \times \mathbb{P}_{1}$ contient $\{a\} \times \mathbb{P}_{1}$. En particulier, $Y \not \subset \Delta$.

L'application

$$
\left.g^{\prime}\right|_{\left(f^{\prime}\right)^{-1}\left(V_{a}^{*}\right)}:\left(f^{\prime}\right)^{-1}\left(V_{a}^{*}\right)=\left(g^{\prime}\right)^{-1}\left(V_{a}^{*} \times \mathbb{P}_{1}\right) \rightarrow V_{a}^{*} \times \mathbb{P}_{1}
$$

est analytique, propre et surjective. On en déduit que $\left(g^{\prime}\right)^{-1}(Y)$ est un sous-ensemble analytique de dimension 1 de $\left(f^{\prime}\right)^{-1}\left(V_{a}^{*}\right)$. Comme $Y \not \subset \Delta$, il résulte du lemme II.3.11, qu'il existe une composante irréductible $Z$ de $\left(g^{\prime}\right)^{-1}(Y)$ invariante par $\mathcal{F}(P, Q)$ et telle que $\operatorname{dim} g^{\prime}(Z)=1$ (voir annexe, lemme A.4.3). Comme $Y$ est irréductible, $g^{\prime}(Z)=Y$.

Finalement, notons $\bar{Z}$ l'adhérence de $Z$ dans $\left(f^{\prime}\right)^{-1}\left(V_{a}\right)$. On voit que $g^{\prime}(\bar{Z})$ est fermé dans $V_{a} \times \mathbb{P}_{1}$. Comme $q \in \bar{Y}, \bar{Z}$ contient un point $p \in$ $\left(g^{\prime}\right)^{-1}(q)$. L'ensemble des points réguliers de $Z$ est contenu dans une feuille de $\left.\mathcal{F}(P, Q)\right|_{\left(f^{\prime}\right)^{-1}\left(V_{a}^{*}\right)}$. Alors, d'après ce qu'on a vu plus haut, $\bar{Z} \cap\left(f^{\prime}\right)^{-1}(a)$ ne contient pas d'autre point que $p$. Cela implique que $\bar{Y} \cap\left(\{a\} \times \mathbb{P}_{1}\right)=\{q\}$, ce qui est une contradiction.

Preuve du théorème. S'il existait une infinité de singularités essentielles de (II.1) il existerait une infinité de valeurs régulières $y$ de $h$ (avec les notations du début de $\S 3)$ telles que $h^{-1}(y)$ est invariante par $\mathcal{F}(P, Q)$, ce qui est absurde : en effet, autrement le diviseur de tangence entre $\mathcal{F}(P, Q)$ et le feuilletage défini par $h$ contiendrait une infinité de fibres de $h$.

\section{II.6 Le point de vue algébrique}

À l'équation (II.1) on peut associer un corps $K$ et une dérivation $D$ sur $K$ (voir définition II.6.1 plus bas). Le corps $K$ est le corps de fractions de l'anneau quotient de $\mathbb{C}[x, y, z]$ par l'idéal engendré par le polynôme $F$ : on a les identifications canoniques $K=\mathbb{C}\left(S_{0}\right)=\mathbb{C}(S)=\mathbb{C}(X)$. La dérivation $D$ est déterminée par

$$
D(c)=0 \text { si } c \in \mathbb{C}, D(x)=1, D(y)=\frac{P(x, y, z)}{Q(x, y, z)} .
$$

Dans ce paragraphe nous allons étudier (II.1) de ce point de vue et déterminer quelles sont les dérivations qui correspondent aux équations dans la classe FP (voir [27] et [11]). 
Dans la section II.6.1 on exposera les préliminaires algébriques et dans les sections II.6.2 et II.6.3, les applications aux feuilletages et aux équations différentielles.

\section{II.6.1 Dérivations et anneaux de valuation}

On supposera donnés trois corps

$$
k \subset L \subset K
$$

Définition II.6.1. Une dérivation sur $K$ est une application $D: K \rightarrow K$ telle que :

$$
D(x+y)=D(x)+D(y) \text { et } D(x y)=x D(y)+y D(x)
$$

pour tous $x, y \in K$.

Les dérivations sur $K$ forment un $K$-espace vectoriel. On notera $\operatorname{Der}_{k}(K / L)$ l'ensemble des dérivations $D$ sur $K$ telles que $D(k)=0$ et

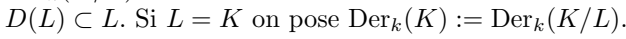

Lemme II.6.2. On a les assertions suivantes :

a) Toute $D \in \operatorname{Der}_{k}(K / L)$ est k-linéaire.

b) $\operatorname{Der}_{k}(K / L)$ est un $L$-espace vectoriel.

c) Soit $D \in \operatorname{Der}_{k}(K)$ et soient $P, Q \in k\left[X_{1}, \ldots, X_{n}\right]$. Supposons que $Q\left(x_{1}, \ldots, x_{n}\right) \neq 0$ pour certains $x_{1}, \ldots, x_{n} \in K$. Si $R\left(X_{1}, \ldots, X_{n}\right):=$ $P\left(X_{1}, \ldots, X_{n}\right) / Q\left(X_{1}, \ldots, X_{n}\right)$, alors

$$
D\left(R\left(X_{1}, \ldots, X_{n}\right)\right)=\sum_{j=1}^{n} \frac{\partial R}{\partial X_{j}}\left(x_{1}, \ldots, x_{n}\right) D\left(x_{j}\right) .
$$

d) Si $L \subset L^{\prime} \subset K$ où $L^{\prime}$ est un corps tel que $L^{\prime} / L$ est une extension algébrique séparable, alors

$$
\operatorname{Der}_{k}(K / L) \subset \operatorname{Der}_{k}\left(K / L^{\prime}\right) .
$$

e) Si $K / L$ est algébrique et séparable, alors l'application $\left.D \mapsto D\right|_{L}$ induit un isomorphisme

$$
\operatorname{Der}_{k}(K / L) \cong \operatorname{Der}_{k}(L)
$$

Démonstration. Les assertions (a), (b) et (c) suivent directement de la définition.

d) Soit $x \in L^{\prime}$. Alors il existe un polynôme $P(X) \in L[X]$ tel que $P(x)=0$ et $P^{\prime}(x) \neq 0$. Si

$$
P(X)=X^{n}+a_{1} X^{n-1}+\cdots+a_{n},
$$


on pose $b_{j}:=D a_{j} \in L, 1 \leq j \leq n$.

Alors, de $D(P(x))=0$ il résulte :

$$
P^{\prime}(x) D x+\left(x^{n}+b_{1} x^{n-1}+\cdots+b_{n}\right)=0 .
$$

On en déduit que $D x \in L^{\prime}$.

e) Voir [36, chap. II, $\S 17$, cor 2']

Définition II.6.3. Un anneau de valuation de $K$ est un sous-anneau $A$ de $K$, avec $A \neq K$, tel que si $x \in K$ et $x \notin A$, alors $x^{-1} \in A$.

Lemme II.6.4. Soit $A$ un anneau de valuation de K. Alors :

a) A contient un unique idéal maximal;

b) A est intégralement clos dans $K$.

Démonstration. Voir [36, chap. VI, $\S 2$, thm. 1 et $\S 4$, thm. 6].

Lemme II.6.5. Supposons que $K$ soit engendré sur L par un nombre fini d'éléments et que le degré de transcendance de $K / L$ soit égal à 1 . Alors, tout anneau de valuation $A$ de $K$ tel que $L \subset A$ est un anneau principal.

Démonstration. Voir [36, chap. VI, $\S 10$, cor.] et [36, chap. VI, $\S 14$, thm. 31 et cor.].

Définition II.6.6. Soit $D \in \operatorname{Der}_{k}(K / L)$. On dira que $D$ est une dérivation

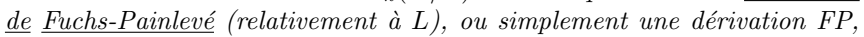
si $\left.D\right|_{L} \neq 0$ et $D(A) \subset A$ pour tout anneau de valuation $A$ de $K$ tel que $L \subset A$.

Proposition II.6.7. Soit $X$ une variété projective complexe, irréductible et non-singulière. Soient $k=\mathbb{C}, K=\mathbb{C}(X)$. Pour chaque champ de vecteurs $u$ méromorphe sur $X$ on définit la dérivation $D_{u} \in \operatorname{Der}_{k}(K)$ par

$$
D_{u}(f):=\frac{\partial f}{\partial u}, f \in K .
$$

Alors, l'application $u \mapsto D_{u}$ est un isomorphisme de $K$-espaces vectoriels de l'espace des champs de vecteurs méromorphes sur $X$ sur l'espace $\operatorname{Der}_{k}(K)$.

Démonstration. Il est clair que l'application $u \mapsto D_{u}$ est $K$-linéaire.

Soient $g_{1}, \ldots, g_{n}$ une base de transcendance de $K / k$ où $n:=\operatorname{dim} X$. Il existe un ouvert de Zariski non-vide $U \subset X$ tel que $g_{1}, \ldots, g_{n}$ est un système de coordonnées locales au voisinage de chaque point de $U$. Alors, si $D_{u}\left(g_{j}\right)=0$ pour $1 \leq j \leq n$, on a $u=0$. 
Soit $D \in \operatorname{Der}_{k}(K)$. Les champs de vecteurs

$$
\frac{\partial}{\partial g_{1}}, \ldots, \frac{\partial}{\partial g_{n}}
$$

sont bien définis et holomorphes sur $U$. Ils se prolongent à des champs méromorphes sur $X$. Définissons

$$
u:=\sum_{j=1}^{n} D\left(g_{j}\right) \frac{\partial}{\partial g_{j}} .
$$

D'après II.6.2c, $D_{u}(g)=D(g)$ si $g \in \mathbb{C}\left(g_{1}, \ldots, g_{n}\right)$. Alors $D=D_{u}$ d'après II. $6.2 \mathrm{e}$.

Proposition II.6.8. Soit $h: X \rightarrow B$ une surface fibrée à fibres connexes où $X$ est une variété projective. Posons

$$
K:=\mathbb{C}(X), L:=h^{*}(\mathbb{C}(B)) \subset K .
$$

a) Soit $S \subset X$ une courbe irréductible telle que $h(S)=B$. Alors l'anneau

$$
A_{S}:=\left\{f \in K: S \not \subset \operatorname{Supp}\left(\operatorname{div}_{\infty} f\right)\right\}
$$

est un anneau de valuation de $K$ qui contient $L$.

b) Soit $A$ un anneau de valuation de $K$ qui contient $L$. Alors, il existe une courbe irréductible $S \subset X$ telle que $h(S)=B$ et $A_{S}=A$.

Avant de prouver II.6.8 nous allons démontrer, avec les mêmes hypothèses et notations, le lemme suivant.

Lemme II.6.9. Soit $\theta \in K, \theta \notin L$. Écrivons le diviseur des zéros de $\theta$ sous la forme

$$
\sum_{j=1}^{r} n_{j} S_{j}+\sum_{i=1}^{t} m_{i} T_{i}
$$

où $h\left(S_{j}\right)=B$ pour $1 \leq j \leq r$ et $\left.h\right|_{T_{i}}=$ cte pour $1 \leq i \leq t$. Considérons $L \subset \mathbb{C}\left(S_{j}\right)$ par restriction et notons $e_{j}:=\left[\mathbb{C}\left(S_{j}\right): L\right], 1 \leq j \leq r$. Alors,

$$
[K: L(\theta)]=\sum_{j=1}^{r} e_{j} n_{j} .
$$

Démonstration. Considérons l'application rationnelle $\phi: X-->B \times \mathbb{P}_{1}$ définie par

$$
\phi(x)=(h(x), \theta(x)), x \in X .
$$


On voit qu'elle est dominante et génériquement finie, puisque $\theta \notin L$. Si $b \in B$ est générique alors

$$
\phi^{-1}(b, 0)=\bigcup_{j=1}^{r}\left(S_{j} \cap h^{-1}(b)\right)
$$

comme ensemble. De plus, si $q \in S_{j} \cap h^{-1}(b)$ la multiplicité de $\phi$ en $q$ est $n_{j}$. On en déduit que

$$
\operatorname{deg} \phi=\sum_{j=1}^{r} e_{j} n_{j},
$$

puisque

$$
e_{j}=\operatorname{deg}\left(\left.h\right|_{S_{j}}\right)=\operatorname{Card}\left(S_{j} \cap h^{-1}(b)\right), 1 \leq j \leq r .
$$

D'autre part, $\phi^{*}\left(\mathbb{C}\left(B \times \mathbb{P}_{1}\right)\right)=L(\theta)$. Alors,

$$
[K: L(\theta)]=\operatorname{deg} \phi=\sum_{j=1}^{r} e_{j} n_{j} .
$$

Preuve de II.6.8. a) Il est clair que si $g \in \mathbb{C}(B)$ alors $g \circ h \in A_{S}$. Donc $L \subset A_{S}$. Comme $X$ est algébrique, il existe $f \in K$ qui a un pôle le long de $S$. Donc, $f \notin A_{S}$. Comme $f^{-1}$ a un zéro le long de $S$, on a $f^{-1} \in A_{S}$.

b) Soit $\mathcal{M}_{A}$ l'idéal maximal de $A$ et soit $\theta \in \mathcal{M}_{A}, \theta \neq 0$. Alors $L[\theta] \subset A$ et $\theta \notin L$. Puisque les fibres de $h$ sont connexes, $L$ est algébriquement clos dans $K$. Donc $L[\theta]$ est un anneau de polynômes en $\theta$ à coefficients dans $L$. Posons

$$
R:=A \cap L(\theta) .
$$

Alors $L[\theta] \subset R$ et $R$ est un anneau de valuation de $L(\theta)$. De plus, $\theta \in \mathcal{M}_{R}$, l'idéal maximal de $R$. Donc $R$ est l'anneau des fractions $P(\theta) / Q(\theta)(P, Q \in$ $L[\theta], Q \neq 0)$ telles que $Q(0) \neq 0$ et $\theta$ est un générateur de $\mathcal{M}_{R}$ ([36, chap. VI, $§ 9$,exa. 3]). Cela implique que

$$
A_{S_{j}} \cap L(\theta)=R(1 \leq j \leq r)
$$

dans les notations de II.6.9.

L'indice de ramification de $A_{S_{j}} / R$ est $n_{j}$ ([36, chap. VI, §11]).

D'autre part, le corps résiduel de $A_{S_{j}}$ est $\mathbb{C}\left(S_{j}\right)$ (par restriction à $S_{j}$ ) et celui de $R$ est $L$. Alors, le degré relatif ([36, chap. VI, §11]) de $A_{S_{j}} / R$ est $e_{j}$. Du lemme II.6.9 et de [36, chap. VI, $\S 11$, thm. 20 et pag. 63] on déduit que $A_{S_{1}}, \ldots, A_{S_{r}}$ sont tous les anneaux de valuation de $K$ dont l'intersection avec $L(\theta)$ est $R$. Donc, $A=A_{S_{i}}$ pour un $i$ entre 1 et $r$. 


\section{II.6.2 Feuilletages et dérivations}

Soit $h: X \rightarrow B$ une surface projective fibrée à fibres connexes. Soit $\Gamma$ le feuilletage de $X$ défini par $h$ (annexe A.2.4). Posons

$$
k:=\mathbb{C}, L:=h^{*}(\mathbb{C}(B)) \subset K:=\mathbb{C}(X) .
$$

Soit $D \in \operatorname{Der}_{k}(K / L)$ avec $D(L) \neq 0$. D'après II.6.7 il existe un champ de vecteurs $u \neq 0$ méromorphe sur $X$ tel que $D=D_{u}$. Soit $\Lambda_{D}$ le feuilletage à singularités isolées de $X$ défini par $u$ (annexe A.3.5).

Théorème II.6.10. On a les assertions suivantes :

a) $\Lambda_{D} \neq \Gamma$.

b) Si $D^{\prime} \in \operatorname{Der}_{k}(K / L)$ et $D^{\prime}(L) \neq 0$, alors $\Lambda_{D}=\Lambda_{D^{\prime}}$ si et seulement si $D^{\prime}=g D, g \in L, g \neq 0$.

c) Si $\Lambda \neq \Gamma$ est un feuilletage de $X$ à singularités isolées, alors il existe $D \in \operatorname{Der}_{k}(K / L)$ telle que $D(L) \neq 0$ et $\Lambda=\Lambda_{D}$.

d) $\Lambda_{D}$ est génériquement transverse (à $h$ : définition II.3.13) si et seulement si $D$ est une dérivation FP (relativement à $L$ : définition II.6.6).

Démonstration. a) Si $\Lambda_{D}=\Gamma$, alors $u$ est tangent aux fibres de $h$. Cela entraîne $\left.D\right|_{L}=\left.D_{u}\right|_{L}=0$.

b) Soit $u^{\prime}$ un champ de vecteurs méromorphe sur $X$ tel que $D^{\prime}=D_{u^{\prime}}$ (proposition II.6.7). Si $\Lambda_{D}=\Lambda_{D^{\prime}}$ alors $u, u^{\prime}$ sont proportionnels. Donc il existe $g \in K, g \neq 0$ telle que $D^{\prime}=g D$.

Soit $f \in L, f \notin \mathbb{C}$. Puisque $L / \mathbb{C}(f)$ est algébrique, $D(f) \neq 0$ et $D^{\prime}(f) \neq 0$ (voir lemme II.6.2e). Alors

$$
g=\frac{D^{\prime}(f)}{D(f)} \in L .
$$

c) Sur $B$ il existe un champ de vecteurs méromorphe $v \neq 0$; pour le voir il suffit de se rappeler que $B$ est revêtement ramifié de $\mathbb{P}_{1}$ : on relève à $B$ un champ de vecteurs méromorphe sur $\mathbb{P}_{1}$.

Comme $\Lambda \neq \Gamma$, il existe un ouvert de Zariski non-vide $U \subset X$ tel que $\left.\Lambda\right|_{U}$ et $\left.\Gamma\right|_{U}$ sont transverses (voir annexe A.5.2) Alors $v$ se relève en un champ de vecteurs $u$ méromorphe sur $U$. Il est facile de voir que $u$ est méromorphe sur $X$ et que le feuilletage défini par $u$ est $\Lambda$ (voir A.3.5 et A.2.6). Alors si $D=D_{u}$ on a $\Lambda=\Lambda_{D}$; il reste donc à prouver que $D(L) \subset L$.

Tout élément de $L$ est de la forme $g \circ h$ où $g \in \mathbb{C}(B)$. Soit $\lambda:=D(g \circ h)=$ $D_{u}(g \circ h)$. Pour prouver que $\lambda \in L$ il suffit de constater que $\lambda$ est holomorphe au voisinage d'une fibre de $h$. En effet, cela implique que les pôles de $\lambda$ sont des composantes irréductibles de quelques fibres. Alors $\lambda$ est constante sur les fibres génériques de $h$ (qui sont compactes et connexes) et passe au quotient dans $B$.

Soit $p \in B$ une valeur régulière de $h$ qui n'est pas un pôle de $g$ telle que $h^{-1}(p) \cap U$ est non-vide et telle que $v$ est holomorphe au voisinage de $p$ 
avec $v(p) \neq 0$. Soit $z$ une coordonnée locale de $B$ centrée en $p$. Fixons un point $q \in h^{-1}(p) \cap U$. Il existe un système de coordonnées locales $(x, y)$ de $X$ centré en $q$ tel que $x=z \circ h$. Dans ces coordonnées :

$$
g \circ h=g(x) \text { et } u=a(x, y) \frac{\partial}{\partial x}+b(x, y) \frac{\partial}{\partial y}
$$

au voisinage de $q$ dans $X$, où $a, b$ sont holomorphes. Observons que

$$
a(x, y)=d x(u)=d z(d h(u))=d z(v) \circ h
$$

ne dépend pas de $x$. Alors la fonction

$$
\frac{\partial(g \circ h)}{\partial u}=a(x) \frac{\partial g}{\partial x}=c(x)
$$

ne dépend que de $x$.

Considérons la fonction $c(z)$, holomorphe dans un voisinage connexe $V_{p}$ de $p$ dans $B$. Alors $c \circ h$ est holomorphe sur $h^{-1}\left(V_{p}\right)$ et coïncide avec $\lambda$ au voisinage de $q$. Donc

$$
\left.\lambda\right|_{h^{-1}\left(V_{p}\right)}=c \circ h,
$$

ce qui prouve que $\lambda$ est holomorphe au voisinage de $h^{-1}(p)$.

d) On reprend les considérations de la preuve de (c) avec $\Lambda=\Lambda_{D}$.

Supposons que $\Lambda_{D}$ soit génériquement transverse à $h$. Alors, on peut supposer que $U$ est le complémentaire d'un nombre fini de fibres de $h$ et que $u$ est holomorphe sur $U$. Soit $S \subset X$ une courbe irréductible telle que $h(S)=B$. Alors $u$ est holomorphe au voisinage de chaque point de $U \cap S$ qui est un ouvert de Zariski non-vide de $S$. On en déduit que $D_{u}\left(A_{S}\right) \subset A_{S}$ (voir II.6.8a). Donc, $D_{u}$ est une dérivation FP d'après II.6.8b, car $D_{u}(L) \subset$ $L$ comme on a vu dans la preuve de (c). De plus, on a vu aussi que $\Lambda_{D}=$ $\Lambda_{D_{u}}$, et alors, d'après (b) on en conclut que $D$ est aussi une dérivation FP.

Réciproquement, supposons que $\Lambda_{D}$ n'est pas génériquement transverse à $h$. Alors il existe une composante irréductible $S$ du support $T$ du diviseur de tangence de $\Lambda_{D}$ et $\Gamma$ telle que $h(S)=B$ (A.5.1 et A.6.2). Précisons que $U=X-T$.

Soit $p \in B$ une valeur régulière de $h$ telle que $v$ est holomorphe au voisinage de $p$ avec $v(p) \neq 0$ et que $h^{-1}(p)$ est transverse à $T$. Fixons $q \in h^{-1}(p) \cap S$. Alors $q$ est régulier dans $S$ et $d h(q)\left(T_{q}(S)\right) \neq 0$. De plus, au voisinage de $q, u$ est holomorphe en dehors de $S$. Comme $X$ est algébrique, il existe $f \in K$ telle que $S$ est un zéro de $f, f$ est holomorphe au voisinage de $q$ et $d f(q) \neq 0$. Alors $x:=z \circ h$ et $y=f$ est un système de coordonnées locales de $X$ centré en $q$ (où $z$ est comme dans la preuve de (c)).

Comme dans la preuve de (c), on voit que

$$
u=a(x) \frac{\partial}{\partial x}+b(x, y) \frac{\partial}{\partial y}
$$


au voisinage de $q$, où $a$ est holomorphe et $b$ est méromorphe avec un pôle le long de $y=0$. Comme

$$
\frac{\partial f}{\partial u}=\frac{\partial y}{\partial u}=b(x, y)
$$

on a que $D_{u}(f) \notin A_{S}$. Donc, $D_{u}$ n'est pas une dérivation FP, d'après II.6.8a. Puisque $\Lambda_{D}=\Lambda_{D_{u}}$ (voir preuve de (c)) on a que $D$ n'est pas une dérivation FP, d'après (b).

Lemme II.6.11. Si $D, D^{\prime} \in \operatorname{Der}_{k}(L)$ sont différentes de 0 , alors il existe $g \in L, g \neq 0$ telle que $D^{\prime}=g D$.

Démonstration. Cela résulte tout de suite de II.6.7 et du fait que $\operatorname{dim} B=$ 1.

Exemple II.6.12. Soit $X=B \times \mathbb{P}_{1} ; h: X \rightarrow B, h^{\prime}: X \rightarrow \mathbb{P}_{1}$ les projections canoniques. On a $\mathbb{C}\left(\mathbb{P}_{1}\right)=\mathbb{C}(z)$. Posons $\theta:=z \circ h^{\prime}$. Alors $K=L(\theta)$ et $\theta$ est transcendante sur $L$.

Fixons $D_{0} \in \operatorname{Der}_{k}(L), D_{0} \neq 0$. D'après le lemme II.6.11 et le théorème II.6.10, tout feuilletage à singularités isolées de $X$ différent de $\Gamma$ est de la forme $\Lambda_{D}$ où $D \in \operatorname{Der}_{k}(K / L)$ et $\left.D\right|_{L}=D_{0}$. D'après [36, chap. II, $\S 17$, cor. 1] la dérivation $D$ est déterminée par la donnée de $\lambda=D(\theta) \in K$. Donc, il suit du théorème II.6.10 que les feuilletages de $X$ à singularités isolées et différents de $\Gamma$ sont en correspondance bijective avec les éléments de $K$; en particulier 0 correspond au feuilletage de $X$ défini par $h^{\prime}$.

Supposons que $\Lambda_{D}$ soit génériquement transverse à $h$. Soit $\lambda=p(\theta) / q(\theta)$ où $p, q \in L[\theta]$. Supposons aussi qu'il existe un polynôme irréductible $r \in$ $L[\theta]$ tel que $r(\theta)$ divise $q(\theta)$ mais ne divise pas $p(\theta)$. Posons

$$
A:=\{a(\theta) / b(\theta): a, b \in L[\theta] \text {, et } r(\theta) \text { ne divise pas } b(\theta)\} .
$$

Alors $L \subset A$ et $A$ est un anneau de valuation de $K$. Mais $\theta \in A$ et $D(\theta) \notin A$, ce qui est impossible d'après le théorème II.6.10d. Il en résulte que $\lambda=$ $p(\theta) \in L[\theta]$.

Considérons maintenant l'ensemble

$$
A:=\{a(\theta) / b(\theta): a, b \in L[\theta], \operatorname{deg} a \leq \operatorname{deg} b\} .
$$

Alors $L \subset A$ et $A$ est un anneau de valuation de $K$. Comme $\theta^{-1} \in A$, on a que

$$
D\left(\theta^{-1}\right)=-\frac{p(\theta)}{\theta^{2}} \in A,
$$

d'après II.6.10d. Donc deg $p \leq 2$. Il résulte de [36, chap. VI, $\S 9$, exa. 3$]$, du théorème II.6.10d et de II.6.2c que l'assertion réciproque est aussi vraie. 


\section{II.6.3 Équations différentielles et dérivations}

On considère maintenant le corps de fractions $K$ de l'anneau quotient de l'anneau de polynômes $\mathbb{C}[x, y, z]$ par l'idéal engendré par $F$, où $F$ est le polynôme de l'équation II.1. Soient $k:=\mathbb{C}$ et $L:=\mathbb{C}(x) \subset K$ (par abus de notation on note encore $x, y, z$ les classes de $x, y, z$ dans $K)$.

Dans les notations du $\S 3$ on a les identifications canoniques

$$
K=\mathbb{C}(X), L=\left(f^{\prime}\right)^{*}\left(\mathbb{C}\left(\mathbb{P}_{1}\right)\right) .
$$

Soit $M:=h^{*}(\mathbb{C}(B))$. Alors, $L \subset M \subset K$ et $M / L$ est algébrique. Donc

$$
\operatorname{Der}_{k}(K / L) \subset \operatorname{Der}_{k}(K / M)
$$

d'après II.6.2d.

À l'équation (II.1) on associe la dérivation $D \in \operatorname{Der}_{k}(K / L)$ déterminée par

$$
D(c)=0 \text { si } c \in k, D(x)=1, D(y)=\frac{P(x, y, z)}{Q(x, y, z)}
$$

(voir [36, chap. II, $\S 17$, thm. 39 et cor. 1] et II.6.2e).

Alors $D \in \operatorname{Der}_{k}(K / M)$. On a vu au début de II.6.2 que $D$ définit un feuilletage $\Lambda_{D}$ de $X$ à singularités isolées différent de celui défini par les fibres de $h$. Dans les notations du $\S 3$ on a le lemme suivant.

Lemme II.6.13. $\Lambda_{D}=\mathcal{F}(P, Q)$.

Démonstration. $\Lambda_{D}$ est défini par un champ de vecteurs $u$ méromorphe sur $X$ tel que $D=D_{u}$ (voir II.6.7). Donc,

$$
\frac{\partial x}{\partial u}=1, \frac{\partial y}{\partial u}=\frac{P(x, y, z)}{Q(x, y, z)} .
$$

D'après la définition de $\mathcal{F}(P, Q)$, ceci implique que $u$ est tangent aux feuilles de $\mathcal{F}(P, Q)$. Donc, $\Lambda_{D}=\mathcal{F}(P, Q)$.

Théorème II.6.14. On a les assertions suivantes :

a) Les classes d'équivalence des équations (II.1) (voir définition II.1.8) sont en correspondance bijective canonique avec l'ensemble des $D \in$ $\operatorname{Der}_{k}(K / L)$ telles que $D(x)=1$.

b) L'équation (II.1) est dans la classe FP si et seulement si la dérivation correspondante est une dérivation FP.

Démonstration. a) Il découle des définitions.

b) Observons que si $A$ est un anneau de valuation de $K$ tel que $L \subset A$, alors $M \subset A$ (voir II.6.4b). Donc (b) se déduit du théorème II.6.10d, du théorème II.3.15 et du lemme II.6.13. 


\section{Chapitre III}

\section{Les équations de genre 0 et de genre $>1$ dans la classe FP}

\section{III.1 Introduction}

Dans ce chapitre on reprend l'étude de l'équation (II.1)

$$
\left\{\begin{array}{l}
P(x, y, z) d x-Q(x, y, z) d y=0 \\
F(x, y, z)=0
\end{array}\right\}
$$

dans les conditions et avec les notations du $\S 1$ du chapitre II.

Dans II.3.6 on a défini la notion de genre de l'équation (II.1). On va maintenant considérer les équations dans la classe FP (voir chap. I $\S 4$ et chap. II $\S 1$ et $\S 3)$ de genre 0 et de genre $>1$. Dans le premier cas on montrera qu'elles se ramènent à des équations de Riccati à coefficients algébriques [29] (l'exemple II.3.16 en est un cas particulier). Comme application on donnera un critère pour l'existence d'une intégrale première rationnelle (voir III.5.7 et III.5.8). Dans le deuxième cas on montrera qu'elles admettent toujours une intégrale première rationnelle (III.6.4 et [29]); l'utilisation des variétés hyperboliques simplifie considérablement la preuve de cette dernière assertion. Ceci a été généralisé par Bruno Scárdua dans $[30]$.

Comme exemple, on montre que les tangentes à une courbe plane irréductible de degré $>1$ sont les solutions d'une équation dans la classe FP de genre égal à celui de la courbe. 


\section{III.2 L'image réciproque régulière}

Soient $h: X \rightarrow B$ une surface fibrée, dans le sens du $\S 3$ du chapitre II et dont les fibres sont connexes, $A$ une surface de Riemann compacte et $\gamma: A \rightarrow B$ une application analytique surjective. En général, l'image réciproque de $h$ par $\gamma$ n'est pas une surface fibrée à cause de l'apparition de singularités.

Définition III.2.1. Une image réciproque régulière de $h$ par $\gamma$ est un triple $(Y, k, \Gamma)$ où :

a) $k: Y \rightarrow A$ est une surface fibrée;

b) $\Gamma: Y \rightarrow X$ est une application analytique telle que $h \circ \Gamma=\gamma \circ k$;

c) il existe un ouvert de Zariski non-vide $V$ de $B$ tel que si $a \in A$ et $\gamma(a) \in V$ alors $\left.\Gamma\right|_{k^{-1}(a)}: k^{-1}(a) \rightarrow h^{-1}(\gamma(a))$ est un isomorphisme.

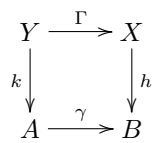

Lemme III.2.2. Il existe toujours une image réciproque régulière de $h$ par $\gamma$.

Démonstration. Soit $h^{\prime}: X^{\prime} \rightarrow A$ l'image réciproque usuelle (c'est-à-dire, le produit fibré : voir [17, chap. II, §3]) de $h$ par $\gamma$ et soit $\gamma^{\prime}: X^{\prime} \rightarrow X$ l'application canonique. Prenons une désingularisation $p: Y \rightarrow X^{\prime}$ de $X^{\prime}$ et posons $\Gamma:=\gamma^{\prime} \circ p$ et $k:=h^{\prime} \circ p$. Alors $(Y, k, \Gamma)$ est une image réciproque régulière de $h$ par $\gamma$; on a le diagramme commutatif

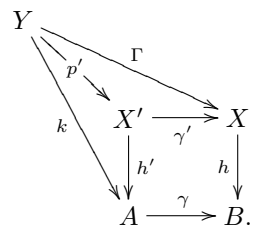

Pour vérifier c) de III.2.1, prenons par $V \subset B$ l'ouvert de Zariski constitué des points qui sont des valeurs régulières de $h$ et de $\gamma$. Alors $W:=$ $\left(h^{\prime}\right)^{-1}\left(\gamma^{-1}(V)\right)$ est constitué de points non-singuliers de $X^{\prime}$. Donc,

$$
\left.p\right|_{p^{-1}(W)}: p^{-1}(W) \longrightarrow W
$$

est un isomorphisme. 
On attire l'attention du lecteur sur le fait que l'application $\Gamma: Y \rightarrow X$ du lemme ne contracte que des composantes irréductibles des fibres de la surface fibrée $k: Y \rightarrow A$, car la surface $X^{\prime}$, obtenue comme image réciproque, n'est singulière que le long d'un nombre fini de fibres de $h^{\prime}$ : $X^{\prime} \rightarrow A$. C'est aussi une conséquence directe de la définition III.2.1 et du fait que $\gamma$ est une application finie.

Lemme III.2.3. Soit $(Y, k, \Gamma)$ une image réciproque régulière de $h$ par $\gamma$. Soit $K \subset X$ une courbe analytique fermée telle que $\left.h\right|_{K}: K \rightarrow B$ est finie. Alors,

$$
\Gamma^{-1}(K)=K^{\prime} \cup K_{1}
$$

où $K^{\prime}$ et $K_{1}$ sont des courbes analytiques fermées, $k_{K^{\prime}}: K^{\prime} \rightarrow A$ est finie et $K_{1} \subset k^{-1}(E)$ où $E \subset A$ est un ensemble fini. Cette décomposition est unique et $\operatorname{deg}\left(\left.h\right|_{K}\right)=\operatorname{deg}\left(\left.k\right|_{K^{\prime}}\right)$. En particulier, si $K$ est une section de $h$, alors $K^{\prime}$ est une section de $k$.

Démonstration. Il découle de la définition III.2.1 puisque $\Gamma$ ne contracte que des composantes irréductibles des fibres de $k$.

Définition III.2.4. On dira que $K^{\prime}$ est l'image réciproque de $K$ dans $Y$.

Lemme III.2.5. Soit $K \subset X$ une courbe analytique fermée telle que $\left.h\right|_{K}$ : $K \rightarrow B$ est finie de degré $\geq 1$. Alors, il existe une surface de Riemann $A$, une application analytique surjective $\gamma: A \rightarrow B$ et une image réciproque régulière $(Y, k, \Gamma)$ de $h$ par $\gamma$ telles que l'image réciproque $K^{\prime}$ de $K$ dans $Y$ est la réunion de d sections différentes de $k$.

Démonstration. Soit $L \subset K$ une composante irréductible qui n'est pas une section. Considérons une désingularisation $\alpha: A \rightarrow L$ de $L$ et notons $\gamma:=h \circ \alpha$. Soient $(Y, k, \Gamma)$ une image réciproque régulière de $h$ par $\gamma$ et $V \subset B$ l'ouvert de la définition III.2.1; posons $U:=\gamma^{-1}(V)$. On peut supposer que tout point de $U$ est une valeur régulière de $k$ et que tout point de $V$ est une valeur régulière de $h$ et de $\gamma$. Dans ces conditions on peut définir une section $s: U \rightarrow Y$ de $k$ par :

$$
\Gamma(s(x))=\alpha(x), x \in U .
$$

Il suit de III.2.1 que

$$
\left.\Gamma\right|_{k^{-1}(U)}: k^{-1}(U) \longrightarrow h^{-1}(V)
$$

est un isomorphisme local. Il en résulte que $s$ est analytique.

Soit $L^{\prime}$ l'image réciproque de $L$ dans $Y$. Alors, $s(U) \subset L^{\prime}$. Donc, si $x \in U$ et $x$ tend vers un point $a \in A$, alors $s(x)$ tend vers l'ensemble $L^{\prime} \cap k^{-1}(a)$, qui est fini. Alors $s(x)$ tend vers un point de $L^{\prime}$. On en déduit que $s$ se prolonge à une section analytique $t: A \rightarrow Y$ de $k$ car $A$ est lisse; de plus $t(A) \subset L^{\prime}$ puisque $L^{\prime}$ est fermé. 
Comme l'image réciproque d'une section est une section, il suffit d'itérer le procédé ci-dessus et d'appliquer III.2.3 pour compléter la démonstration.

Proposition III.2.6. Supposons que $X$ soit une surface projective. Soit d un entier positif. Alors, il existe une surface de Riemann A, une application analytique surjective $\gamma: A \rightarrow B$ et une image réciproque régulière $(Y, k, \Gamma)$ de $h$ par $\gamma$ telles que $k: Y \rightarrow A$ possède d sections analytiques différentes.

Démonstration. Puisque $X$ est une variété projective, il résulte du théorème de Bertini (voir [17, chap. II, thm. 8.18]) qu'il existe dans $X$ une infinité de courbes irréductibles dont l'auto-intersection est positive. Aucune de ces courbes n'est contenue dans une fibre de $h$, par le lemme de Zariski ([2, chap. III, lem. 8.2]).

De ce qui précède on déduit qu'il existe une courbe algébrique projective $K \subset X$ telle que $\left.h\right|_{K}: K \rightarrow B$ est finie de degré $\geq d$. La proposition découle, alors, de III.2.5

Rappelons que si $W$ est un espace analytique irréductible, $\mathbb{C}(W)$ désigne le corps de fonctions méromorphes sur $T$.

Lemme III.2.7. Soit $(Y, k, \Gamma)$ une image réciproque régulière de $h$ par $\gamma$; comme $\gamma, \Gamma, h, k$ sont surjectives et $h \circ \Gamma=\gamma \circ k$, on peut supposer que $\mathbb{C}(A), \mathbb{C}(B)$ et $\mathbb{C}(X)$ sont des sous-corps de $\mathbb{C}(Y)$. Alors $\mathbb{C}(Y)$ est engendré, comme corps, par les éléments de $\mathbb{C}(A)$ et $\mathbb{C}(X)$.

Démonstration. Soit $d:=\operatorname{deg} \gamma$. D'après la définition III.2.1, $\Gamma$ est génériquement finie et $\operatorname{deg} \Gamma=d$. Alors,

$$
[\mathbb{C}(Y): \mathbb{C}(X)]=d .
$$

Par ailleurs, comme $h$ est à fibres connexes, $\mathbb{C}(B)$ est algébriquement clos dans $\mathbb{C}(X)$. Si $f \in \mathbb{C}(A)$ est un élément primitif de l'extension $\mathbb{C}(A) / \mathbb{C}(B)$, on en déduit que

$$
\begin{aligned}
d & =[\mathbb{C}(B)[f]: \mathbb{C}(B)] \\
& =[\mathbb{C}(X)[f]: \mathbb{C}(X)] \\
& \leq[\mathbb{C}(Y): \mathbb{C}(X)],
\end{aligned}
$$

d'où l'assertion.

Définition III.2.8. On dira que la surface fibrée $h: X \rightarrow B$ est birationnellement triviale s'il existe une surface de Riemann compacte $\bar{F}$ et une application birationnelle $f: B \times F \rightarrow X$ telle que $p=h \circ f$ où $p: B \times F \rightarrow B$ est la projection canonique. On dit que f est une trivialisation birationnelle de $h$. 
Observation III.2.9. Cela implique que $f$ ne contracte que des composantes irréductibles de quelques fibres et que les fibres de $h$ sont génériquement isomorphes à $F$.

\section{III.3 Résultats géométriques}

Soit $K$ le diviseur canonique d'une surface projective lisse $X$ et soit $C \subset$ $X$ une courbe algébrique projective irréductible. On rappelle les résultats suivants :

A) Formule d'adjonction : $2 g(C)-2=C \cdot(K+C)-2 \delta(C)$

où $g(C)$ est le genre (d'une désingularisation) de $C$, le point désigne l'indice d'intersection dans $X$ et $\delta(C) \geq 0$ est un entier qui s'annule si et seulement si $C$ est non-singulière ([3, I- 15 et $\mathrm{I}-16])$.

B) Si $h: X \rightarrow B$ est une surface fibrée et $C \subset h^{-1}(b)$ pour un $b \in B$, alors $C \cdot C \leq 0$ et $C \cdot C=0$ implique $C=h^{-1}(b)$. De plus, l'auto-intersection de chaque fibre de $h$, en tant que diviseur, est nulle [2, chap. III, lem. 8.2].

Proposition III.3.1. Soit $h: X \rightarrow B$ une surface fibrée telle que:

a) il existe une fibre régulière isomorphe à $\mathbb{P}_{1}$;

b) aucune fibre ne contient de courbe isomorphe à $\mathbb{P}_{1}$ d'auto-intersection -1. Alors $h$ est un fibré localement analytiquement trivial de fibre type $\mathbb{P}_{1}$.

Démonstration. Prenons $b \in B$ et posons $F:=h^{-1}(b)$.

Fixons $F_{0}=h^{-1}\left(b_{0}\right)$ une fibre régulière isomorphe à $\mathbb{P}_{1}$; par A) et B)

$$
-2=F_{0} \cdot K
$$

Par ailleurs, il existe une fonction $f$ méromorphe et non-constante sur $B$ dont le seul pôle est $b$ et dont les zéros $b_{1}, \ldots, b_{s}$ sont tous simples et sont aussi des valeurs régulières de $h$. Si $C_{1}, \ldots, C_{k}$ sont les composantes irréductibles de $F$, alors

$$
\operatorname{div}(f \circ h)=\sum_{j=1}^{s} h^{-1}\left(b_{j}\right)-s \sum_{i=1}^{k} n_{i} C_{i}, n_{i}>0 .
$$

On en déduit que le diviseur

$$
D=\sum_{i=1}^{k} n_{i} C_{i}
$$

est numériquement équivalent à $F_{0}$. Donc,

$$
\sum_{i=1}^{k} n_{i} C_{i} \cdot K=D \cdot K=F_{0} \cdot K=-2
$$

On distingue deux cas : 
Cas 1. Supposons que l'une des courbes $C_{i}$ soit d'auto-intersection nulle. Par B), $k=1$ et $D=n C$ avec $C \cdot C=0$. Comme $n C \cdot K=-2$, on a $n=1$ ou $n=2$.

Si $n=1$, alors $C \cdot K=-2$. Par A), $g(C)=\delta(C)=0$ et $F$ est isomorphe à $\mathbb{P}_{1}$.

Si $n=2$, alors $C \cdot K=-1$. Mais A) implique que $C \cdot K$ est pair, ce qui est une contradiction.

Cas 2. Supposons que $C_{i} \cdot C_{i}<0$ pour tout $i=1, \ldots k$ (voir B) ci-dessus). En appliquant A) à chaque $C_{i}$, multipliant par $n_{i}$ et faisant la somme on obtient, compte tenu de (III.1), que :

$$
-2=\sum_{i=1}^{k} n_{i}\left(2 g\left(C_{i}\right)-2-C_{i} \cdot C_{i}+2 \delta\left(C_{i}\right)\right) .
$$

Si $C_{i} \cdot C_{i}<-1$, alors le terme correspondant dans cette somme est $\geq 0$. Si $C_{i} \cdot C_{i}=-1$ et $g\left(C_{i}\right)=\delta\left(C_{i}\right)=0$, la courbe $C_{i} \subset F$ serait isomorphe à $\mathbb{P}_{1}$, ce qui contredit l'hypothèse. Si $C_{i} \cdot C_{i}=-1$ et $g\left(C_{i}\right)+\delta\left(C_{i}\right)>0$, le terme correspondant dans la somme est $>0$. On obtient $-2 \geq 0$; contradiction.

En définitive, on a démontré que $D=C$ où $C$ est une courbe isomorphe à $\mathbb{P}_{1}$. C'est-à-dire, toutes les fibres sont régulières et isomorphes à $\mathbb{P}_{1}$. Cela implique la proposition d'après [12]

Si $a, b, c, d$ sont quatre points d'une surface de Riemann de genre 0 , on désigne $[a, b, c, d]$ leur birapport.

Lemme III.3.2. Supposons que la surface fibrée $h: X \rightarrow B$ admet trois sections analytiques différentes $s_{0}, s_{1}, s_{2}$ et que ses fibres régulières sont isomorphes à $\mathbb{P}_{1}$. Alors, il existe une trivialisation birationnelle $f: B \times$ $\mathbb{P}_{1} \rightarrow X$ de $h$ telle que les images de $B \times\{0\}, B \times\{1\}, B \times\{\infty\}$ par $f$ sont $s_{0}(B), s_{1}(B), s_{2}(B)$ respectivement.

Démonstration. En contractant les éventuelles composantes irréductibles des fibres isomorphes à $\mathbb{P}_{1}$ et d'auto-intersection -1 on peut se réduire, par III.3.1, au cas où $h$ est une fibration analytiquement localement triviale. Dans ce cas on définit une application $g: X \rightarrow B \times \mathbb{P}_{1}$ par :

$$
\left[s_{0}(y), s_{1}(y), s_{2}(y), x\right]=[0,1, \infty, g(x)]
$$

où $x \in X, y=h(x)$. L'inverse $f$ de $g$ est la trivialisation birationnelle cherchée.

L'existence de sections pour une surface fibrée est, en général, difficile à établir; de plus, il n'est pas toujours vrai qu'une surface fibrée, même 
que sans fibre singulière possède une section. Cependant, dans le cas où les fibres régulières sont isomorphes à $\mathbb{P}_{1}$, il existe toujours une section : ceci est la version géométrique d'un résultat connu comme Théorème de Tsen (on peut consulter [31, chap. I, cor. 4]). Par un argument standard de cohomologie de faisceaux on peut montrer que l'existence d'une section implique celle d'une infinité de sections; du lemme suit alors le résultat suivant, connu comme Théorème de Nöther-Enriques (voir [3, chap.III]).

Théorème III.3.3. Soit $h: X \rightarrow B$ une surface fibrée dont les fibres régulières soient isomorphes à $\mathbb{P}_{1}$ et supposons que $X$ soit une surface algébrique. Alors $h$ est birationnellement triviale.

Observation III.3.4. Si $K \subset X$ est une section de $h$ on peut choisir la trivialisation birationnelle de sorte que $B \times\{\infty\}$ coïncide avec $K$.

\section{III.4 Les équations de genre zéro}

Dans ce paragraphe on montrera que si l'équation (II.1) est de genre 0, alors son étude se ramène à celle d'un feuilletage d'un produit $A \times \mathbb{P}_{1}$, où $A$ est une surface de Riemann compacte, différent de celui donné par les fibres de la projection $A \times \mathbb{P}_{1} \rightarrow A$ (c'est-à-dire qu'elle se ramène au cas des équations étudiées dans [18, chap. 3]). Si de plus l'équation est dans la classe $\mathrm{FP}$, ce feuilletage est génériquement transverse à cette projection canonique (dans le sens de II.3.13) : l'étude de ces feuilletages est le sujet du paragraphe suivant. Ces résultats sont à comparer avec $[6$, chap. $4, \S 1]$

Proposition III.4.1. Supposons que l'équation (II.1) soit de genre 0. Alors, il existe une surface de Riemann compacte $A$, une application analytique surjective $\gamma: A \rightarrow \mathbb{P}_{1}$ et une application rationnelle $\Gamma: A \times$ $\mathbb{P}_{1}-->\mathbb{P}_{1}^{3}$ telles que le diagramme suivant

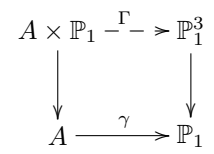

commute, où les flèches verticales sont les projections canoniques sur le premier facteur. De plus, pour $a \in A$ générique, $\gamma(a) \in \mathbb{C}$ et $\Gamma$ applique birationnellement $\{a\} \times \mathbb{P}_{1}$ sur une composante irréductible de la courbe

$$
\{F(\gamma(a), y, z)=0\} \subset\{\gamma(a)\} \times \mathbb{P}_{1}^{2} .
$$

Démonstration. Considérons la surface fibrée $h: X \rightarrow B$ introduite au $\S 3$ du chapitre II dont on reprend les notations et définitions; on pose $A=B$. 
Par hypothèse, les fibres régulières sont isomorphes à $\mathbb{P}_{1}$. On est, donc, en mesure d'appliquer III.3.3. Tenant compte de II.3.4 et de III.2.9, la proposition suit immédiatement.

Observation III.4.2. On peut exprimer III.4.1 de façon informelle en disant que si l'équation est de genre 0 , alors on peut paramétrer birationnellement la courbe $F(x, y, z)=0$, à $x$ fixé, par des fonctions

$$
y=A(x, t), z=B(x, t)
$$

rationnelles en $t$ et à coefficients des fonctions algébriques en $x$. Cette paramétrisation est birationnelle pour $x$ générique.

Exemple III.4.3. $F(x, y, z)=z^{2}-x y^{2}$. Il n'existe pas $A, B$ comme dans III.4.2 qui soient des fonctions rationnelles en $t$ et en $x$, car autrement on aurait

$$
B(x, t)^{2}=x A(x, t)^{2},
$$

ce qui est absurde; le problème ici est que la courbe $F(a, y, z)=0$ est réductible pour $a$ générique; après avoir pris une image réciproque par une application de degré 2 , selon le théorème de factorisation de Stein ([17, chap. III, cor. 11.5]), on trouvera de telles $A, B$, rationnelles en $t$ mais avec des coefficients des fonctions algébriques (de degré 2 ).

Reprenons les notations et définitions du début du $\S 3$ du chapitre II et supposons que l'équation soit de genre zéro. Soient $A, \gamma$ et $\Gamma$ comme dans III.4.1. Soit

$$
\Gamma^{\prime}: A \times \mathbb{P}_{1}-->\mathbb{P}_{1}^{2}
$$

la composition de $\Gamma$ avec la projection canonique :

$$
\mathbb{P}_{1}^{3}=\mathbb{P}_{1}^{2} \times \mathbb{P}_{1} \longrightarrow \mathbb{P}_{1}^{2}
$$

Théorème III.4.4. On a les assertions suivantes :

a) L'équation de Pfaff dans $A \times \mathbb{P}_{1}$

$$
\Gamma^{*}(P(x, y, z) d x-Q(x, y, z) d y)=0
$$

n'est pas triviale; notons $\mathcal{G}$ le feuilletage analytique à singularités isolées de $A \times \mathbb{P}_{1}$ défini par cette équation.

b) $\mathcal{G}$ est différent du feuilletage défini par les fibres de la projection canonique $A \times \mathbb{P}_{1} \rightarrow A$;

c) pour $a \in \mathbb{C}$ générique et pour tout $b \in \mathbb{P}_{1}$, si $\Sigma \not \subset \Delta$ est le support d'une solution de (II.1) par $q=(a, b)$, il existe une séparatrice locale $\Lambda$ de $\mathcal{G}$ en $p \in A \times \mathbb{P}_{1}$ telle que $\Gamma$ est définie en $p, \Gamma^{\prime}(p)=q$ et $\Gamma^{\prime}(\Lambda)=\Sigma$;

d) si $C \not \subset \Delta$ est une solution algébrique de (II.1), il existe une courbe algébrique projective irréductible $K \subset A \times \mathbb{P}_{1}$ invariante par $\mathcal{G}$ telle que l'image de $K$ par $\Gamma^{\prime}$ est l'adhérence de $C$ dans $\mathbb{P}_{1}^{2}$; 
e) s'il existe dans $A \times \mathbb{P}_{1}$ une infinité de courbes algébriques irréductibles fermées invariantes par $\mathcal{G}$, l'équation (II.1) possède une intégrale première rationnelle;

f) si l'équation (II.1) est dans la classe FP, $\mathcal{G}$ est génériquement transverse à la projection canonique $A \times \mathbb{P}_{1} \rightarrow A$.

Démonstration. a) Par construction, $\Gamma$ est une application rationnelle dominante :

$$
\Gamma: A \times \mathbb{P}_{1}-->S .
$$

De ceci et de II.3.3 on déduit que

$$
\Gamma^{*}(P(x, y, z) d x-Q(x, y, z) d y)
$$

est une 1-forme méromorphe et non-nulle sur $A \times \mathbb{P}_{1}$.

b) L'assertion résulte du fait que $F$ ne divise pas $Q$.

c) Considérons le diagramme commutatif (voir chap. II, §3)

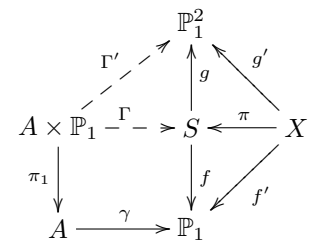

où $\pi_{1}$ est la projection canonique.

Comme $\pi^{-1} \circ \Gamma$ ne contracte que des composantes de quelques fibres de $\pi_{1}$, il existe un ouvert de Zariski non-vide $W_{2} \subset \mathbb{C} \subset \mathbb{P}_{1}$ tel que si $W_{1}:=\gamma^{-1}\left(W_{2}\right)$, alors :

$$
\Theta:=\left.\pi^{-1} \circ \Gamma\right|_{\pi_{1}^{-1}\left(W_{1}\right)}: \pi_{1}^{-1}\left(W_{1}\right) \longrightarrow\left(f^{\prime}\right)^{-1}\left(W_{2}\right)
$$

est analytique, propre et finie. D'après III.4.1, pour $a \in W_{1}$ générique, $\left.\Gamma\right|_{\pi_{1}^{-1}(a)}$ est une désingularisation d'une composante irréductible de la courbe $F(a, y, z)=0$ tandis que $\left.\pi\right|_{\left(f^{\prime}\right)^{-1}(\gamma(a))}$ est une désingularisation de cette courbe (II.2.2). Il en résulte que $\left.\Theta\right|_{\pi_{1}^{-1}(a)}$ est injective. On en déduit que $\Theta$ est un isomorphisme local, à condition de prendre $W_{2}$ plus petit si nécessaire (c'est-à-dire que $W_{2}$ ne contient pas de valeur singulière de $h$ ).

Rappelons que $\mathcal{F}(P, Q)$ est le feuilletage de $X$ associé à l'équation (II.1) (voir $\S 3$ du chapitre II).

D'après les définitions, l'image réciproque par $\Theta$ de $\left.\mathcal{F}(P, Q)\right|_{\left(f^{\prime}\right)^{-1}\left(W_{2}\right)}$ est $\left.\mathcal{G}\right|_{\pi_{1}^{-1}\left(W_{1}\right)}$ (voir II.3.3). Il suffit, alors, d'appliquer II.3.11.

d) Il est analogue à c) au moyen de II.3.12.

e) D'après ce qu'on a vu dans la preuve de c), si $C \subset A \times \mathbb{P}_{1}$ est une courbe irréductible invariante par $\mathcal{G}$ et telle que $\pi_{1}(C)=A$, alors son image par 
$\pi^{-1} \circ \Gamma$ est une courbe irréductible invariante par $\mathcal{F}(P, Q)$ (voir A.4.3 dans l'annexe). Par le théorème de Jouanolou [21], l'hypothèse entraîne que $\mathcal{F}(P, Q)$ possède une intégrale première rationnelle. On conclut par II.3.20.

f) Si l'équation est dans la classe $\mathrm{FP}, \mathcal{F}(P, Q)$ est transverse aux fibres génériques de $f^{\prime}$ (II.3.15 et II.3.14). Comme $\Theta$ est un isomorphisme local, cela implique que $\mathcal{G}$ est transverse aux fibres génériques de $\pi_{1}$.

\section{III.5 Les équations de Riccati}

Soit $A$ une surface de Riemann compacte et supposons donné un feuilletage analytique à singularités isolées $\mathcal{G}$ de $Y:=A \times \mathbb{P}_{1}$ différent de celui défini par les fibres de la projection canonique $p: Y \rightarrow A$.

Comme $Y$ est une variété algébrique, $\mathcal{G}$ est donné par une équation de Pfaff globale $\theta=0$, où $\theta$ est une 1-forme méromorphe non-nulle sur $Y$ (voir annexe A.3.4). Soit $t: Y \rightarrow \mathbb{P}_{1}$ l'autre projection canonique. Choisissons $l \in \mathbb{C}(A)$ non-constante et soit $x:=l \circ p$. Alors,

$$
\theta=u d x+v d t
$$

où $u, v \in \mathbb{C}(Y)$ et $v \neq 0$. On peut, donc, écrire l'équation sous la forme

$$
\frac{d t}{d x}=f, f=-\frac{u}{v} \in \mathbb{C}(Y) .
$$

Lemme III.5.1. Si $\mathcal{G}$ est génériquement transverse à p, alors

$$
f=a t^{2}+b t+c
$$

où $a, b, c \in \mathbb{C}(A)$.

Démonstration. Voir [18, thm.3.3.3] ou exemple II.6.12.

Observation III.5.2. Supposons que l'équation (II.1) soit de genre 0 et soit

$$
y=A(x, t), z=B(x, t)
$$

comme dans III.4.2. L'équation du feuilletage $\mathcal{G}$ de $Y$ défini au théorème III.4.4 est :

$$
\begin{array}{r}
P(x, A(x, t), B(x, t)) d x-Q(x, A(x, t), B(x, t)) A_{x}(x, t) d x \\
-Q(x, A(x, t), B(x, t)) A_{t}(x, t) d t=0 .
\end{array}
$$

Il en résulte l'équation explicite :

$$
\frac{d t}{d x}=\frac{P(x, A(x, t), B(x, t))-Q(x, A(x, t), B(x, t)) A_{x}(x, t)}{Q(x, A(x, t), B(x, t)) A_{t}(x, t)} .
$$


Si, en plus, l'équation (II.1) est dans la classe FP, celle-là est une équation de Riccati, d'après III.5.1 et III.4.4f (comparer avec II.3.16).

Exemple III.5.3. $\left(y^{\prime}\right)^{2}-x y(x-y)^{2}=0$. Comme $F(x, y, z)=z^{2}-x y(x-y)^{2}$, la courbe $F(a, y, z)=0$ est une cubique avec un point double en $y=a$, $z=0$. L'équation est donc de genre 0 .

Comme $y=0$ est une solution, il résulte de I.4.11, I.4.13 et I.2.6 que l'équation est dans la classe FP. On pose

$$
y=P(x, t)=t^{2}, z=Q(x, t)=t \sqrt{x}\left(x-t^{2}\right)
$$

et on obtient l'équation de Riccati :

$$
\frac{d t}{d x}=-\frac{\sqrt{x}}{2} t^{2}+\frac{x \sqrt{x}}{2} .
$$

Observons qu'on a perdu la solution $y=0$, contenue dans $\Delta$.

Lemme III.5.4. Supposons que $\mathcal{G}$ soit génériquement transverse à $p$. Soit $U \subset A$ un ouvert et soient $y=y_{j}(x), 1 \leq j \leq 4$, des fonctions analytiques sur $U$, à valeurs dans $\mathbb{P}_{1}$ telles que leurs graphes dans $U \times \mathbb{P}_{1} \subset Y$ sont disjoints deux à deux et invariants par $\mathcal{G}$. Alors, le birapport

$$
\left[y_{1}(x), y_{2}(x), y_{3}(x), y_{4}(x)\right]
$$

est constant pour $x \in U$.

Démonstration. D'après II.3.14, on peut supposer que $\mathcal{G}$ est transverse à $p^{-1}(x)$ pour tout $x \in U$. Comme le problème est local, on peut prendre $U$ assez petit pour que $\mathcal{G}$ trivialise le fibré $\left.p\right|_{p^{-1}(U)}$. C'est-à-dire, il existe

$$
\lambda: p^{-1}(U) \longrightarrow \mathbb{P}_{1}
$$

analytique, telle que $\left.\lambda\right|_{p^{-1}(x)}$ est un isomorphisme pour $x \in U$ et que $\lambda(u)=$ $\lambda(v)$ si et seulement si $u, v \in p^{-1}(U)$ sont dans la même feuille de $\left.\mathcal{G}\right|_{p^{-1}(U)}$ (voir annexe A.6.3). Alors,

$$
\begin{aligned}
& {\left[y_{1}(x), y_{2}(x), y_{3}(x), y_{4}(x)\right]} \\
& =\left[\lambda\left(x, y_{1}(x)\right), \lambda\left(x, y_{2}(x)\right), \lambda\left(x, y_{3}(x)\right), \lambda\left(x, y_{4}(x)\right)\right] \\
& =\text { cte. }
\end{aligned}
$$

parce que $\lambda\left(x, y_{j}(x)\right)$ est constante pour $j=1,2,3,4$.

Exemple III.5.5. Dans les hypothèses et notations de III.5.1, supposons que $A \times\{\infty\}$ soit invariante par $\mathcal{G}$. Alors $a=0$; c'est-à-dire, on a une équation linéaire. 
Lemme III.5.6. Supposons que $\mathcal{G}$ soit génériquement transverse à $p$ et qu'il existe une courbe algébrique projective $K \subset Y$ telle que :

a) $K \cap p^{-1}(x)$ est fini pour tout $x \in A$,

b) le degré de $\left.p\right|_{K}: K \rightarrow A$ est $\geq 3$,

c) $K$ est invariante par $\mathcal{G}$.

Alors, il existe dans $Y$ une infinité de courbes algébriques projectives irréductibles invariantes par $\mathcal{G}$.

Démonstration. Il est évident qu'on peut supposer que $A \times\{\infty\} \not \subset K$. Alors, l'hypothèse implique qu'il existe un ouvert connexe et non-vide $U \subset$ $A$, et des fonctions $y_{j}=y_{j}(x)(j=1,2,3)$ analytiques sur $U$ à valeurs dans $\mathbb{C}$ dont leurs graphes dans $U \times \mathbb{P}_{1} \subset Y$ sont disjoints deux à deux et invariants par $\mathcal{G}$. On peut aussi supposer que $\left.\mathcal{G}\right|_{p^{-1}(U)}$ n'a pas de point singulier.

Dans ces conditions, il suit de III.5.4 et du théorème d'existence et unicité pour les équations différentielles analytiques, que le graphe de la fonction analytique $y=y(x)$ définie, pour $c \in \mathbb{C}$ générique, par l'expression

$$
\left[y_{1}(x), y_{2}(x), y_{3}(x), y(x)\right]=c, x \in U,
$$

est invariant par $\mathcal{G}$.

Finalement, puisque les $y_{j}(x) \in \mathbb{C}(U)$ sont algébriques sur $\mathbb{C}(A) \subset \mathbb{C}(U)$, la fonction $y(x) \in \mathbb{C}(U)$ l'est aussi. Donc, il existe une courbe algébrique fermée irréductible dans Y qui contient ce graphe; cette courbe est invariante par $\mathcal{G}$, d'où suit le lemme.

Théorème III.5.7. Supposons que :

a) l'équation (II.1) soit de genre 0 et appartienne à la classe FP,

b) il existe une courbe algébrique $C \subset \mathbb{C}^{2}$ dont toutes les composantes irréductibles sont des solutions algébriques telle que son équation minimale est de degré $\geq 3$ en $y$ et que $C \cap \Delta$ est fini.

Alors, l'équation (II.1) admet une intégrale première rationnelle.

Démonstration. Il résulte de III.4.4f, III.4.4d, III.5.6 et III.4.4e.

Exemple III.5.8. $\left(y^{\prime}\right)^{2}-y^{2}\left(1-y^{2}\right)=0$. Comme $F(x, y, z)=z^{2}-y^{2}\left(1-y^{2}\right)$, la courbe $F(a, y, z)=0$ est une quartique irréductible avec deux points doubles. L'équation est donc de genre 0. De I.4.11, I.4.13 et I.2.6 on déduit que cette équation est dans la classe FP. Suivant III.5.2 on prend

$$
y=P(x, t)=\frac{2 t}{1+t^{2}}, z=Q(x, t)=\frac{2 t\left(1-t^{2}\right)}{\left(1+t^{2}\right)^{2}}
$$

et on obtient l'équation : $d t / d x=t$. On en déduit que la solution générale est de la forme :

$$
y=\frac{2 c e^{x}}{1+c^{2} e^{2 x}}, c \in \mathbb{C} \text { et } y= \pm 1 .
$$


Il est facile de voir que $y=0, \pm 1$ sont les seules solutions algébriques.

Cette équation possède trois solutions algébriques différentes mais n'admet pas d'intégrale première rationnelle. Observons que toutes les composantes irréductibles de la courbe $C$ d'équation $y^{3}-y=0$ sont des solutions, tandis que $C \cap \Delta$ n'est pas fini.

Complément III.5.9. Supposons que l'équation (II.1) appartienne à la classe FP faible (voir I.4.3), que son genre est 0 et que

$$
\operatorname{deg}_{y} c_{1}(x, y) \geq \operatorname{deg}_{y} c_{j}(x, y), 2 \leq j \leq m .
$$

Si $\operatorname{deg}_{y} c_{1}(x, y)=0$, l'équation est dans la classe FP puisque, pour $a \in \mathbb{C}$ générique, il n'existe pas de solution par $(a, \infty)$.

Supposons $\operatorname{deg}_{y} c_{1}(x, y)>0$. Dans ce cas $L \subset S$, dans les notations du $\S 3$, chap. II, où $L$ est la droite :

$$
L:=\mathbb{P}_{1} \times\{(\infty, \infty)\} \subset \mathbb{P}_{1}^{3} .
$$

De plus, pour $a \in \mathbb{C}$ générique, $(a, \infty, \infty) \in L$ est non-singulier dans $S$. Il en résulte que $\pi^{-1}(L)$ est composé d'une section $T$ de $f^{\prime}$ et d'éventuelles courbes contenues dans des fibres de $f^{\prime}$ (l'existence de cette section entraîne $B=\mathbb{P}_{1}$ et $\varphi=I d$; c'est-à-dire, $F(a, y, z)=0$ est une courbe irréductible pour $a \in \mathbb{C}$ générique).

D'après III.3.3 et III.3.4 il existe une trivialisation birationnelle $\alpha: \mathbb{P}_{1} \times$ $\mathbb{P}_{1} \rightarrow X$ de $h^{\prime}$ telle que l'image de $A \times\{\infty\}$ par $\alpha$ est l'image réciproque de $T$ (définitions III.2.1 et III.2.4). De ceci on déduit que le feuilletage $\mathcal{G}$ de $\mathbb{P}_{1} \times \mathbb{P}_{1}$ défini dans III.4.4 est transverse à la fibration $\mathbb{P}_{1} \times \mathbb{P}_{1} \rightarrow A$ en dehors d'un ensemble de la forme

$$
\mathbb{P}_{1} \times\{\infty\} \cup E \times \mathbb{P}_{1}
$$

où $E \subset \mathbb{P}_{1}$ est fini. Il en résulte, comme au début de ce paragraphe, que l'équation se ramène à une équation de la forme :

$$
\frac{d t}{d x}=a_{0}(x) t^{n}+a_{1}(x) t^{n-1}+\cdots+a_{n}(x)
$$

où les $a_{j}(x)$ sont des fonctions algébriques, au moyen d'un changement de variables du type :

$$
y=A(x, t), z=B(x, t)
$$

où $A, B$ sont des fonctions rationnelles en $t$ à coefficients fonctions rationnelles en $x$.

Exemple III.5.10. On reprend l'équation binomiale, dans la classe FP, considérée dans l'exemple I.4.6 (voir[20, §13.8]). On a vu dans II.3.7 que cette équation est de genre 0 dans les cas A), B), C), D), E).

Dans les cas A), B), C), l'équation se ramène immédiatement à une équation de Riccati dont les coefficients sont des fonctions algébriques de 
$x$. Dans le cas D), suivant ce qu'on vient de voir, on doit paramétrer la courbe d'équation

$$
z^{2 n}=B(x)(p(x) y+q(x))^{2 n}\left(y-b_{2}\right)^{n}\left(y-b_{3}\right)^{n}
$$

comme dans III.4.2. L'égalité

$$
z^{2}=\sqrt[n]{B(x)}(p(x) y+q(x))^{2} \frac{y-b_{2}}{y-b_{3}}\left(y-b_{3}\right)^{2}
$$

suggère de faire

$$
t^{2}=\left(y-b_{2}\right) /\left(y-b_{3}\right) .
$$

Un calcul direct montre que, en effet, ce changement de variable dépendante ramène l'équation à l'équation de Riccati :

$$
\frac{d t}{d x}=\frac{1}{2} \sqrt[2 n]{B(x)}\left(b_{3} p(x)+q(x)\right) t^{2}-\left(b_{2} p(x)+q(x)\right) .
$$

On traite E) de manière analogue en faisant

$$
t^{d}=\frac{y-b_{1}}{y-b_{2}} .
$$

et on obtient l'équation de Riccati en variables séparées :

$$
\frac{d t}{d x}=\sqrt[d n]{B(x)} \frac{b_{1}-b_{2}}{d} t^{2}
$$

\section{III.6 Les équations de genre $\geq 2$}

Le théorème suivant est un cas particulier du théorème III.6.2 plus bas compte tenu de II.3.14.

Théorème III.6.1. Soit $h: X \rightarrow B$ une surface fibrée et soit $\mathcal{F}$ un feuilletage analytique de $X$, génériquement transverse à $h$. Si la fibre générique de $h$ est de genre $\geq 2$, alors $\mathcal{F}$ possède une intégrale première méromorphe.

Pour la notion d'holonomie d'un feuilletage on renvoie le lecteur à [7] (voir aussi A.6.6).

Théorème III.6.2. Soient $h: X \rightarrow B$ une surface fibrée et $S \subset B$ un ensemble fini; notons $V:=B-S, U:=h^{-1}(V)$. Soit $\mathcal{F}$ un feuilletage analytique de $U$, transverse à $F_{y}:=h^{-1}(y)$ pour $y \in V$. Si les fibres régulières de $h$ sont de genre $\geq 2$, alors $\mathcal{F}$ se prolonge à un feuilletage analytique $\mathcal{G}$ de $X$ qui possède une intégrale première méromorphe. 
Démonstration. On peut supposer que $S$ contient toutes les valeurs critiques de $h$. Fixons $a \in V$. Comme les fibres de $h$ sont compactes, l'holonomie de $\mathcal{F}$ nous donne un homomorphisme

$$
\pi_{1}(V, a) \longrightarrow \operatorname{Aut}\left(F_{a}\right)
$$

(voir annexe, §6) Étant donné que le groupe $\operatorname{Aut}\left(F_{a}\right)$ des automorphismes de $F_{a}$ est fini, car le genre de $F_{a}$ est $\geq 2$ ([17, chap. IV, exa. 5.2]), il existe un revêtement fini non-ramifié $\alpha: V^{\prime} \rightarrow V$ tel que l'holonomie du relèvement $\mathcal{F}^{\prime}$ de $\mathcal{F}$ à $U^{\prime}$ soit triviale, où $g: U^{\prime} \rightarrow V^{\prime}$ est l'image réciproque par $\alpha$ de $\left.h\right|_{U}: U \rightarrow V$. Ce revêtement peut être complété en un revêtement ramifié fini $\beta: B^{\prime} \rightarrow B$; notons $h^{\prime}: X^{\prime} \rightarrow B^{\prime}$ l'image réciproque de $h: X \rightarrow B$ par $\beta$.

Soit $q: Y \rightarrow X^{\prime}$ une désingularisation de $X^{\prime}$. Comme l'holonomie de $\mathcal{F}^{\prime}$ est triviale, ce feuilletage induit une trivialisation analytique du fibré $g: U^{\prime} \rightarrow V^{\prime}$; c'est-à-dire, il existe une application analytique $p: U^{\prime} \rightarrow F_{a}$ qui identifie chaque fibre de $g$ avec $F_{a}$ et qui est constante sur chaque feuille de $\mathcal{F}^{\prime}$ (voir A.6.6). D'après [22, chap IV, cor. 4.13] et [22, chap VI, teo. 4.1], l'application $p \circ\left(\left.q\right|_{q^{-1}\left(U^{\prime}\right)}\right)$ se prolonge à une application analytique $r: Y \rightarrow F_{a}$.

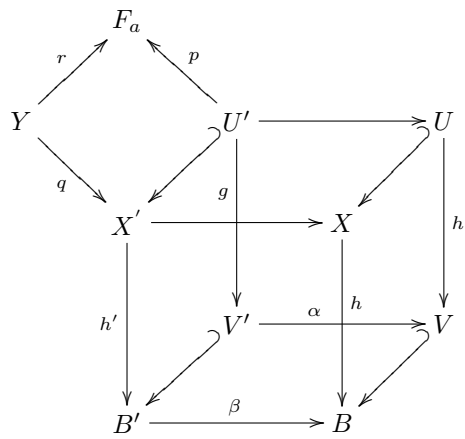

Prenons une fonction méromorphe $u$ non-constante sur $F_{a}$ et considérons $v:=u \circ r$. Alors $v$ est une fonction méromorphe qui est, d'une part, nonconstante sur $Y$, donc aussi sur $X^{\prime}$, et, d'autre part, constante sur chaque feuille de $\mathcal{F}^{\prime}$.

Puisque $X^{\prime}$ est un revêtement fini de $X$, le corps $\mathbb{C}\left(X^{\prime}\right)$ est une extension algébrique finie de $\mathbb{C}(X)$. Comme $v \in \mathbb{C}\left(X^{\prime}\right)$ n'est pas constante, au moins l'un des coefficients, disons $f$, de son polynôme caractéristique sur $\mathbb{C}(X)$ n'est pas constant. Alors $d f=0$ définit un feuilletage de $X$ (annexe, $\S 3$ ) qui prolonge $\mathcal{F}$, parce que $f$ est constante sur chaque feuille de $\mathcal{F}$, ce qui termine la preuve. 
Exemple III.6.3. Soit $B:=\mathbb{C} \cup\{\infty\}, X:=B \times B, h: X \rightarrow B$ la projection canonique sur le premier facteur. Dans $\mathbb{C} \times \mathbb{C} \subset X$ le champ de vecteurs

$$
z^{2} \frac{\partial}{\partial z}+e^{z} \frac{\partial}{\partial w}
$$

engendre un feuilletage qui se prolonge à $\mathbb{C} \times B$ en ajoutant la feuille $\mathbb{C}^{*} \times\{\infty\}$ et le point singulier $(0, \infty)$. Ce feuilletage est transverse aux fibres de $\left.h\right|_{\mathbb{C}^{*} \times\{\infty\}}$. Mais le champ de droites tangentes aux feuilles n'a pas de limite lorsqu'on tend vers un point quelconque de $\{\infty\} \times \mathbb{C}$. Donc, ce feuilletage ne se prolonge pas à $X$.

Théorème III.6.4. Supposons que l'équation (II.1) soit dans la classe FP et que son genre est $>1$. Alors, il existe une intégrale première rationnelle de (II.1).

Démonstration. Il résulte de II.3.15, II.3.20 et III.6.1.

Exemple III.6.5. Soit $C_{0} \subset \mathbb{C}^{2}$ une courbe irréductible de degré $d>1$. Soit $U \subset C_{0}$ l'ensemble des points réguliers $q$ de $C_{0}$ tels que la tangent à $C_{0}$ en $q$ n'est pas verticale. Soit $y=y_{q}(x)$ l'équation de cette tangente. L'application $T: \mathbb{C} \times U \rightarrow \mathbb{C}^{3}$ définie par

$$
T(x, q)=\left(x, y_{q}(x), y_{q}^{\prime}(x)\right)
$$

définit une application rationnelle

$$
T: \mathbb{P}_{1} \times U--\rightarrow \mathbb{P}_{1}^{3},
$$

où $C$ est l'adhérence de $C_{0}$ dans $\mathbb{P}_{2}$, dont l'image est une surface algébrique irréductible fermée $S \subset \mathbb{P}_{1}^{3}$. Soit $S_{0}:=S \cap \mathbb{C}^{3}$ et soit $F(x, y, z)=$ 0 une équation irréductible de $S_{0}$. Si $a \in \mathbb{P}_{1}$, l'application $\left.T\right|_{\{a\} \times C}$ est génériquement injective. C'est-à-dire, pour $a \in \mathbb{C}$ générique $C$ est birationnellement équivalente à la courbe $F(a, y, z)=0$.

De ce qui précède on déduit que $F\left(x, y, y^{\prime}\right)=0$ est une équation de genre égal au genre de $C$. Si $q \in U$, alors $y=y_{q}(x)$ est une solution. On va montrer que l'équation est dans la classe FP.

Il existe un ouvert de Zariski non-vide $V$ de $S$ tel que pour tout $p \in V$, il existe $x \in \mathbb{C}$ et $q \in U$ avec :

$$
p=\left(x, y_{q}(x), y_{q}^{\prime}(x)\right) .
$$

Soit $W$ l'intersection de $V$ avec l'ouvert $\left\{F_{z}(x, y, z) \neq 0\right\}$. Alors, par I.1.3 et ce qu'on vient de voir, pour tout $p=(a, b, c) \in W$, la seule solution par $(a, b)$ de pente $c$ est l'équation d'une droite, à équivalence près.

Considérons, d'autre part, la courbe $\Sigma:=S-W$. Soit $f: S \rightarrow \mathbb{P}_{1}$ la projection sur la première coordonnée. Pour $a \in \mathbb{C}$ générique, si $p \in \Sigma$ et $f(p)=a$, alors $p$ est régulier dans $\Sigma$ et $d\left(\left.f\right|_{\Sigma}\right)(p) \neq 0$. 
Soit $x=\alpha(t), y=\beta(t)$ une solution locale en $a \in \mathbb{C}$. Posons

$$
\gamma(t)=\left(\alpha(t), \beta(t), \beta^{\prime}(t) / \alpha^{\prime}(t)\right) \in S
$$

Alors, pour $a \in \mathbb{C}$ générique, si $\gamma(t) \in \Sigma$ pour tout $t$, la solution est nonramifiée ( II.3.2). Si, par contre, il existe $t_{0}$ tel que $\gamma\left(t_{0}\right) \in W$, alors :

$$
\beta(t)=k \alpha(t)+l
$$

où $k$ et $l$ sont des constantes. La solution est encore non-ramifiée.

Par conséquent, pour chaque entier non-négatif $g$ il existe une équation de genre $g$ dans la classe FP. 


\section{Chapitre IV}

\section{Les équations de genre 1 dans la classe FP}

\section{IV.1 Introduction}

Dans ce chapitre on étudiera les équations du type de l'équation (II.1) qui sont dans la classe FP, lorsque le genre est 1 . On montrera que dans ce cas, l'équation (II.1) se ramène, via un changement de variables adéquat, à une équation aux variables séparées (voir aussi [29]). Comme application on établit un critère pour l'existence d'une intégrale première rationnelle (voir IV.4.5 et IV.3.3).

\section{IV.2 Résultats géométriques}

Soit $T$ est un espace analytique irréductible. Si $T$ est non-singulier et $g \in \mathbb{C}(T), g \neq 0$, on écrit

$$
\operatorname{div} g:=\operatorname{div}_{0} g-\operatorname{div}_{\infty} g
$$

où $\operatorname{div}_{0} g$ est le diviseur de zéros de $g$ et $\operatorname{div}_{\infty} g$ celui des pôles de $g$.

Une surface fibrée (voir §II.3) est relativement minimale si aucune de ses fibres ne contient de courbe lisse rationnelle d'auto-intersection -1 (voir [2, chap. III, §10]; quitte à contracter un nombre fini de ces courbes, on peut factoriser toute surface fibrée à travers une surface fibrée à fibres connexes relativement minimale, via la factorisation de Stein ([17, chap. III, cor. $11.5])$.

Lemme IV.2.1. Soit $X$ une surface algébrique, soit $h: X \rightarrow B$ une surface fibrée dont les fibres régulières sont elliptiques et supposons qu'il existe une section holomorphe $\sigma: B \rightarrow X$ de $h$. Alors, il existe $t, v \in \mathbb{C}(X)$ 
telles que:

$$
\operatorname{div}_{\infty} t=2 \sigma(B)+D \text { et } \operatorname{div}_{\infty} v=3 \sigma(B)+D^{\prime}
$$

où les supports des diviseurs $D, D^{\prime}$ sont contenus dans l'union d'un nombre fini de fibres de $h$.

Démonstration. Sans perte de généralité on peut supposer $h$ relativement minimale. Soit $K$ un diviseur canonique de $X$. Par la formule de Kodaira ([2, chap. V, cor. 12.3]) le support de $K$ est contenu dans $h^{-1}(E)$, où $E \subset B$ est un ensemble fini, et $K \cdot K=0$ (où le point indique l'indice d'intersection dans $X)$. Soit $F$ une fibre régulière de $h, F \not \subset h^{-1}(E)$. Considérons le diviseur

$$
D_{m, n}=m C+K+n F
$$

où $n, m$ sont des entiers positifs et $C:=\sigma(B)$. Alors, par la dualité de Serre :

$$
\chi\left(D_{m, n}\right)=h^{0}\left(D_{m, n}\right)-h^{1}(-m C-n F)+h^{0}(-m C-n F)
$$

où $\chi$ dénote la caractéristique d'Euler-Poincaré et $h^{i}$ les nombres de Betti $\mathrm{du}$ faisceau associé au diviseur; évidemment, $h^{0}(-m C-n F)=0$.

On vérifie facilement que si $m$ est donné, alors le diviseur $m C+n F$ est 1-connexe (dans le sens de [2, chap. II, §12]) et son auto-intersection est positive pour $n$ assez grand. Il en résulte que :

$$
h^{1}(-m C-n F)=0,
$$

d'après [2, chap.IV, cor. 8.2$]$. On en déduit que pour chaque $m \in \mathbb{N}$ :

$$
h^{0}\left(D_{m, n}\right)=\chi\left(D_{m, n}\right)
$$

pour tout $n \in \mathbb{N}$ assez grand.

D'autre part, par Riemann-Roch :

$$
\chi\left(D_{m, n}\right)=\frac{1}{2}\left[m^{2} C \cdot C+m(2 n+K \cdot C)\right]+\chi_{X}
$$

où le dernier terme est la caractéristique du faisceau structurel de $X$. Donc,

$$
\chi\left(D_{m+1, n}\right)-\chi\left(D_{m, n}\right)=\frac{2 m+1}{2} C \cdot C+n+\frac{1}{2} K \cdot C .
$$

Alors, pour chaque $m \in \mathbb{N}, h^{0}\left(D_{m+1, n}\right)>h^{0}\left(D_{m, n}\right)$ pour tout $n \in \mathbb{N}$ assez grand. En particulier, il existe $n \in \mathbb{N}$ tel que :

$$
h^{0}\left(D_{3, n}\right)>h^{0}\left(D_{2, n}\right)>h^{0}\left(D_{1, n}\right),
$$

d'où aussitôt, le fait qu'il existe $t, v \in \mathbb{C}(X)$ tels que

$$
\operatorname{div} t+D_{2, n} \text { et } \operatorname{div} v+D_{3, n}
$$


sont effectifs mais

$$
\operatorname{div} t+D_{1, n} \text { et } \operatorname{div} v+D_{2, n}
$$

ne le sont pas. On voit facilement que $t, v$ satisfont les conditions voulues.

Lemme IV.2.2. Dans les mêmes hypothèses de IV.2.1, on suppose encore $\mathbb{C}(B) \subset \mathbb{C}(X)$ par composition avec $h$. Alors, il existe $t, u \in \mathbb{C}(X)$ tels que : a) si $F$ est une fibre générique de $h,\left.t\right|_{F}: F \rightarrow \mathbb{P}_{1}$ est holomorphe de degré 2 et $\infty$ est l'une de ses valeurs critiques;

b) $u^{2}=P(t)$, où $P(t)$ est un polynôme séparable de degré trois à coefficients dans $\mathbb{C}(B)$;

c) $\mathbb{C}(X)$ est engendré par $t$ et $u$ sur $\mathbb{C}(B)$, autrement dit

$$
\mathbb{C}(X)=\mathbb{C}(B)(t)[u] .
$$

Démonstration. Soient $t, v$ comme dans IV.2.1. Alors $\left.t\right|_{F}: F \rightarrow \mathbb{P}_{1}$ est holomorphe de degré 2 (avec un seul pôle) si $F$ est une fibre générique de $h$. Donc, l'application rationnelle :

$$
h \times t: X-->B \times \mathbb{P}_{1}
$$

est dominante de degré 2 . On en déduit que :

$$
[\mathbb{C}(X): \mathbb{C}(B)(t)]=2 .
$$

Alors,

$$
\mathbb{C}(X)=\mathbb{C}(B)(t)[v]
$$

car $v \notin \mathbb{C}(B)(t)$ : en effet, le pôle de $\left.v\right|_{F}$ est d'ordre 3 d'après IV.2.1.

Soit $v^{\prime}$ le conjugué de $v$ comme élément de l'extension quadratique

$$
\mathbb{C}(X) / \mathbb{C}(B)(t)
$$

posons $u:=v-v^{\prime}$. On voit que

$$
\mathbb{C}(X)=\mathbb{C}(B)(t)[u] \text { et } u^{2} \in \mathbb{C}(B)(t) ;
$$

de plus, si $F$ est une fibre générique de $h$ et si $x, x^{\prime} \in F$ sont tels que $t(x)=t\left(x^{\prime}\right)$ alors

$$
u(x)+u\left(x^{\prime}\right)=0 .
$$

En effet, si $\alpha: X \rightarrow X$ est l'automorphisme rationnel qui permute les points d'une fibre générique de $h \times t$, on a $(u \circ \alpha)^{2}=u^{2}$. C'est-à-dire, $u \circ \alpha= \pm u$; mais $u \circ \alpha=u$ implique $u \in \mathbb{C}(B)[t]$.

Écrivons

$$
u^{2}=\frac{P(t)}{Q(t)} \text { avec } P(t), Q(t) \in \mathbb{C}(B)[t] .
$$


Puisque $F$ est elliptique, $\left(\left.u\right|_{F}\right)^{2}$ est un polynôme séparable de degré 3 en $\left.t\right|_{F}$ à coefficients complexes. On en déduit, d'abord, que le reste de la division de $P$ par $Q$ dans $\mathbb{C}(B)[t]$ est nul. On peut, donc, supposer que $u^{2}=P(t)$. On voit, ensuite, que $P$ est séparable et de degré 3 .

Théorème IV.2.3. Soit $h: X \rightarrow B$ une surface fibrée dont les fibres génériques soient isomorphes et de genre 1 et telle que $X$ est une variété algébrique. Alors, il existe une surface de Riemann compacte A, une application analytique finie $\gamma: A \rightarrow B$ et une image réciproque régulière $(Y, k, \Gamma)$ de $h$ par $\gamma$ (déf. III.2.1) avec les propriétés suivantes :

a) il existe $t \in \mathbb{C}(Y)$ et $\delta \in \mathbb{C}, \delta \neq 0,1$, tels que si $F$ est une fibre générique de $k$, alors $\left.t\right|_{F}: F \rightarrow \mathbb{P}_{1}$ est holomorphe de degré 2 et ses valeurs critiques sont $0,1, \infty, \delta$;

b) il existe $u \in \mathbb{C}(Y)$ tel que

$$
u^{2}=t(t-1)(t-\delta)
$$

c) si on considère $\mathbb{C}(A) \subset \mathbb{C}(Y)$ par composition avec $k$, le corps $\mathbb{C}(Y)$ est engendré par $t$ et $u$ sur $\mathbb{C}(A)$, autrement dit

$$
\mathbb{C}(Y)=\mathbb{C}(A)(t)[u] .
$$

Démonstration. Par III.2.6 on peut supposer que $h$ admet une section analytique $\sigma: B \rightarrow X$. Soient $t, u \in \mathbb{C}(X)$ les fonctions méromorphes données par IV.2.2. Choisissons $l \in \mathbb{C}(B)$ non-constante et posons $g:=l \circ h$. Alors,

$$
t, g: X-->\mathbb{P}_{1}
$$

sont des applications rationnelles, holomorphes sur un ouvert $U \subset X$ tel que $X-U$ est un ensemble fini. Considérons :

$$
L:=\{x \in U: d t(x) \text { et } d g(x) \text { ne sont pas indépendantes }\} .
$$

Alors $L$ est un sous-ensemble analytique de $U$ dont l'adhérence dans $X$ est un sous-ensemble analytique de $X$. Il en résulte qu'il existe une courbe algébrique projective $K \subset X$ telle que :

i) $\left.h\right|_{K}: K \rightarrow B$ est finie,

ii) si $F$ est une fibre générique de $h$, alors $F \cap K$ est l'ensemble des points critiques de $\left.t\right|_{F}$. Par la formule de Hurwitz, $\left.t\right|_{F}$ a quatre points critique. Donc deg $\left.h\right|_{K}=4$.

D'après III.2.5, il existe une surface de Riemann compacte $A$, une application analytique finie $\gamma: A \rightarrow B$ et une image réciproque régulière $(Y, k, \Gamma)$ de $h$ par $\gamma$, telle que l'image réciproque $K^{\prime}$ de $K$ dans $Y$ (définition III.2.4) est formée par quatre sections différentes $\sigma_{0}, \sigma_{1}, \sigma_{2}, \sigma_{\infty}$ de $k$. Par abus, on 
désigne $t \circ \Gamma$ et $u \circ \Gamma$ encore par $t$ et $u$ respectivement; on a $t, u \in \mathbb{C}(Y)$. Maintenant, si $F_{a}:=k^{-1}(a)$ avec $a \in A$ générique, alors

$$
\left.t\right|_{F_{a}}: F_{a} \rightarrow \mathbb{P}_{1}
$$

est holomorphe de degré 2 et ses points critiques sont :

$$
\sigma_{0}(a), \sigma_{1}(a), \sigma_{2}(a), \sigma_{\infty}(a)
$$

où $t\left(\sigma_{\infty}(a)\right)=\infty$. En plus,

$$
\mathbb{C}(Y)=\mathbb{C}(A)(t)[u],
$$

d'après III.2.7, et

$$
u^{2}=p t^{3}+q t^{2}+r t+s
$$

avec $p, q, r, s \in \mathbb{C}(A)$ et $p \neq 0$. Puisque $F_{a}$ est elliptique, les racines de l'équation

$$
p(a) t^{3}+q(a) t^{2}+r(a) t+s(a)=0
$$

sont $\sigma_{0}(a), \sigma_{1}(a), \sigma_{2}(a)$. En effet

$$
2 u d u=\left(3 p(a) t^{2}+2 q(a) t+r(a)\right) d t .
$$

Comme le polynôme $p(a) t^{3}+q(a) t^{2}+r(a) t+s(a)$ est séparable, on en déduit que $d t \neq 0$ implique $u \neq 0$.

Soit $t_{0}:=t \circ \sigma_{0} \in \mathbb{C}(A)$. En remplaçant $t$ par $t-t_{0} \circ k$ on voit qu'on peut supposer que $t_{0}=0$; posons $t_{1}:=t \circ \sigma_{1}$. En remplaçant $t$ par $t /\left(t_{1} \circ k\right)$ on voit qu'on peut supposer que $t_{0}=0$ et $t_{1}=1$. Alors, d'après l'hypothèse et $[17$, chap IV, $\S 4]$ :

$$
t_{2}:=t \circ \sigma_{2}=\delta
$$

où $\delta$ est une constante différente de 0 et 1 . Donc,

$$
u^{2}=p t(t-1)(t-\delta) \text {. }
$$

En prenant, éventuellement, une nouvelle image réciproque régulière on peut supposer que $p=m^{2}$ avec $m \in \mathbb{C}(A)$ (voir III.2.7). Pour compléter la preuve on remplace $u$ par $u / m$.

\section{IV.3 Les équations de genre 1 dans la classe FP}

Dans ce paragraphe on montrera comment ramener les équations de genre 1 qui sont dans la classe FP à des feuilletages d'un produit $A \times E$, où $A$ est une surface de Riemann compacte et $E$ est une courbe elliptique, qui sont génériquement transverse à la projection canonique $A \times E \rightarrow A$. Dans le $\S 4$, on déterminera ces feuilletages (comparer avec [6, chap. $3, \S 3]$ ). 
Proposition IV.3.1. Supposons que l'équation (II.1) soit de genre 1 et appartienne à la classe FP. Alors, il existe une surface de Riemann compacte $A$, une application holomorphe finie $\lambda: A \rightarrow \mathbb{P}_{1}$, un nombre complexe $\delta \neq 0,1$ et une application rationnelle $\Lambda: A \times E-\rightarrow \mathbb{P}_{1}^{3}$, où $E \subset \mathbb{P}_{2}$ est la courbe elliptique d'équation affine :

$$
u^{2}=t(t-1)(t-\delta)
$$

avec les propriétés suivantes :

a) le diagramme

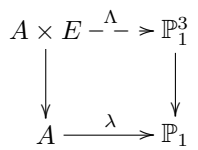

commute, ou les flèches verticales sont les projections canoniques sur le premier facteur;

b) pour tout $a \in A$ générique, $\lambda(a) \in \mathbb{C}$ et $\Lambda$ applique birationnellement $E:=\{a\} \times E$ sur une composante irréductible de la courbe

$$
\{F(\gamma(a), y, z)=0\} \subset\{\gamma(a)\} \times \mathbb{P}_{1}^{2} .
$$

Démonstration. On reprend les considérations de II, §3. La surface fibrée $h: X \rightarrow B$ satisfait aux hypothèses de IV.2.3 parce que les fibres régulières sont elliptiques (voir II.3.4 et II.3.5) et que le feuilletage est transverse aux fibres génériques (II.3.13, II.3.14 et II.3.15; voir aussi $\S 6$ de l'annexe). Soient $A, \gamma, \delta$ et $(Y, k, \Gamma)$ donnés par IV.2.3. Considérons l'application rationnelle $\theta: Y--\rightarrow A \times E$ définie par

$$
\theta(y)=(k(y),(t(y), u(y))) .
$$

D'après IV.2.3c, $\theta$ est birationnelle. On définit :

$$
\Lambda:=\pi \circ \Gamma \circ \theta^{-1}, \lambda:=\varphi \circ \gamma
$$

(voir diagramme (II.3)). On conclut par II.3.4

Observation IV.3.2. La proposition IV.3.1 nous dit que l'on peut paramétrer les courbes $F(x, y, z)=0$, où l'on considère $x=$ cte., par des fonctions :

$$
\begin{aligned}
& y=A(x, t)+B(x, t) \sqrt{t(t-1)(t-\delta)}, \\
& z=C(x, t)+D(x, t) \sqrt{t(t-1)(t-\delta)}
\end{aligned}
$$

où $A, B, C, D$ sont des fonctions rationnelles en $t$ à coefficients des fonctions algébriques en $x$. 
Reprenons les notations et définitions du chap. II, $\S 3$, et supposons que l'équation est de genre 1 et qu'elle appartient à la classe FP. Soient $A, \lambda, \Lambda, \delta$ et $E$ comme dans la proposition IV.3.1. Soit

$$
\Lambda^{\prime}: A \times E-->\mathbb{P}_{1}^{2}
$$

la composition de $\Lambda$ avec la projection canonique :

$$
\mathbb{P}_{1}^{3}=\mathbb{P}_{1}^{2} \times \mathbb{P}_{1} \longrightarrow \mathbb{P}_{1}^{2}
$$

$P, Q \in \mathbb{C}[x, y, z]$ désignent encore les polynômes considérés dans l'équation (II.1) du chap. II, $\S 1$.

Théorème IV.3.3. On a les assertions suivantes :

a) L'équation de Pfaff dans $A \times E$

$$
\Lambda^{*}(P(x, y, z) d x-Q(x, y, z) d y)=0
$$

n'est pas triviale; notons $\mathcal{G}$ le feuilletage analytique à singularités isolées de $A \times E$ défini par cette équation.

b) $\mathcal{G}$ est génériquement transverse à la projection canonique $A \times E \rightarrow A$;

c) pour $a \in \mathbb{C}$ générique et pour tout $b \in \mathbb{P}_{1}$, si $\Sigma \not \subset \Delta$ est le support d'une solution de (II.1) par $q=(a, b)$, il existe une séparatrice locale $\Omega$ de $\mathcal{G}$ en un point $p \in A \times E$ telle que $\Lambda^{\prime}$ est définie en $p, \Lambda^{\prime}(p)=q$ et $\Lambda^{\prime}(\Omega)=\Sigma$;

d) si $C \not \subset \Delta$ est une solution algébrique de (II.1), il existe une courbe algébrique projective irréductible $K \subset A \times E$ invariante par $\mathcal{G}$ telle que l'image de $K$ par $\Lambda^{\prime}$ est l'adhérence de $C$ dans $\mathbb{P}_{1}^{2}$;

e) s'il existe dans $A \times E$ une infinité de courbes algébriques irréductibles fermées invariantes par $\mathcal{G}$, l'équation (II.1) possède une intégrale première rationnelle;

Démonstration. On déduit IV.3.3 de IV.3.1 par le même raisonnement de la preuve de III.4.4.

\section{IV.4 Séparation de variables}

Soient $A$ une surface de Riemann compacte, $E \subset \mathbb{P}_{2}$ la courbe elliptique définie par l'équation affine :

$$
u^{2}=t(t-1)(t-\delta), \delta \in \mathbb{C}-\{0,1\},
$$

et $\mathcal{G}$ un feuilletage analytique à singularités isolées de $Y:=A \times E$.

Soient $p: Y \rightarrow A, q: Y \rightarrow E$ les projections canoniques. Par composition avec $p, q$ on considère $\mathbb{C}(A) \subset \mathbb{C}(Y)$ et $\mathbb{C}(E) \subset \mathbb{C}(Y)$ (dans les notations du début du §2). 
Comme $Y$ est une variété algébrique, $\mathcal{G}$ est défini par une équation de Pfaff $\theta=0$ où $\theta$ est une 1-forme méromorphe sur $Y$, non-nulle (annexe, $\S 3)$.

Rappelons que $d t / u$ est une 1-forme holomorphe sur $E$.

Proposition IV.4.1. Soit $x \in \mathbb{C}(A)$ non-constante. Si $\mathcal{G}$ est génériquement transverse à $p$ (déf. II.3.13) alors :

$$
\theta=f d x-\frac{d t}{u} \text { avec } f \in \mathbb{C}(A) .
$$

Démonstration. On a

$$
\theta=g d x-h \frac{d t}{u}, g, h \in \mathbb{C}(Y) .
$$

Comme $\mathcal{G}$ n'est pas la fibration $p, h \neq 0$. Alors, on peut supposer :

$$
\theta=f d x-\frac{d t}{u}, f \in \mathbb{C}(Y) .
$$

Prenons $a \in A$ générique. D'une part, $x$ est holomorphe au voisinage de $a$ et $a$ n'est pas un point critique de $x$; d'autre part, la fibre $E_{a}:=p^{-1}(a)$ est transverse aux feuilles de $\mathcal{G}$ (II.3.14), n'est pas contenue dans le diviseur de pôles de $f$ et ne contient aucun des points où l'application rationnelle $f: Y \rightarrow \mathbb{P}_{1}$ n'est pas définie.

Par ailleurs, supposons que $\xi \in E_{a}$ soit un pôle de $\left.f\right|_{E_{a}}$. Alors,

$$
\left(\left.((1 / f) \theta)\right|_{E_{a}}\right)(\xi)=0,
$$

ce qui implique que $\mathcal{G}$ est tangent à $E_{a}$ au point $\xi$; contradiction. Il résulte que $\left.f\right|_{E_{a}}$ est constante. Donc $f \in \mathbb{C}(A)$.

Observation IV.4.2. On déduit de IV.4.1 et IV.3.3 qu'un changement de variables de la forme indiquée dans IV.3.2 ramène l'équation (II.1) à une équation de la forme :

$$
f(x) d x=\frac{d t}{\sqrt{t(t-1)(t-\delta)}}, \delta \in \mathbb{C}-\{0,1\}
$$

où $f$ est une fonction algébrique de $x$.

Exemple IV.4.3. $x^{2}\left(y^{\prime}\right)^{2}-2 x y y^{\prime}+\left(y^{2}-4 y^{3}+x^{2} y\right)=0$. On a

$$
D(x, y)=4 x^{2} y\left(4 y^{2}-x^{2}\right) .
$$

Comme $y=0, x / 2,-x / 2$ sont des solutions de l'équation, de I.4.11, I.4.13 et I.2.6 suit que celle-ci est dans la classe FP. Si $a \in \mathbb{C}$ et $a \neq 0$, la courbe plane d'équation

$$
a^{2} z^{2}-2 a y z+\left(y^{2}-4 y^{3}+a^{2} y\right)=0
$$


est une cubique non-singulière. Donc, l'équation est de genre 1 . On fait $y=t x$ et on obtient :

$$
y=t x, z=t+\sqrt{x} \sqrt{4 t^{3}-t} .
$$

Par ce changement de variables on obtient l'équation :

$$
\frac{d t}{\sqrt{x}}=\frac{d t}{\sqrt{4 t^{3}-t}} .
$$

Si $\wp(z)$ est la fonction de Weierstrass associée à l'équation

$$
u^{2}=4 t^{3}-t
$$

l'intégration de l'équation précédente nous donne :

$$
t=\wp(2 \sqrt{x}+c), c \in \mathbb{C} .
$$

Donc, la solution générale de l'équation donnée est :

$$
y=x \wp(2 \sqrt{x}+c), c \in \mathbb{C}, y=0, x / 2,-x / 2 .
$$

La double périodicité de la fonction $\wp(z)$ implique que l'équation donnée n'a pas d'intégrale première rationnelle.

Lemme IV.4.4. Supposons qu'il existe une courbe algébrique projective irréductible $K \subset Y$ invariante par $\mathcal{G}$ et telle que $\left.p\right|_{K}$ est finie. Alors, il existe une infinité de telles courbes.

Démonstration. D'après IV.4.1, l'équation de définition de $\mathcal{G}$ est invariante par les transformations :

$$
(a, b) \mapsto(a, T(a))
$$

où $T$ est une translation de $E$.

Théorème IV.4.5. Supposons que l'équation (II.1) soit de genre 1 et appartienne à la classe FP. S'il existe une solution algébrique qui n'est pas contenue dans $\Delta$, alors l'équation possède une intégrale première rationnelle.

Démonstration. La preuve suit de IV.3.3c, IV.4.4 et IV.3.3d.

Exemple IV.4.6. $\left(1-x^{4}\right)\left(y^{\prime}\right)^{2}-\left(1-y^{4}\right)=0$. Puisque $y=1,-1, i,-i$ sont des solutions, on vérifie par I.4.11, I.4.13 et I.2.6 que l'équation est dans la classe FP. Si $c \neq 0$, la quartique d'équation

$$
c z^{2}-1+y^{4}=0
$$


n'a qu'un point singulier à l'infini. On voit tout de suite que son genre est 1.

Par ailleurs, on a la solution $y=x$ qui n'est pas contenue dans $\Delta$.

Par séparation des variables on obtient :

$$
\int_{0}^{y} \frac{d u}{\sqrt{1-u^{4}}} \pm \int_{0}^{x} \frac{d u}{\sqrt{1-u^{4}}}=c t e
$$

La Formule d'Addition d'Euler ([32, chap. I, §2]) nous donne l'intégrale première :

$$
4 x^{2} y^{2}\left(1-x^{4}\right)\left(1-y^{4}\right)-\left[\lambda^{2}\left(1+x^{2} y^{2}\right)-x\left(1-y^{4}\right)-y\left(1-x^{4}\right)\right]^{2}=0 .
$$

Exemple IV.4.7. On reprend l'équation binomiale dans la classe FP de l'exemple I.4.17 (comparer avec $[20, \S 13.8]$ ). Dans II.3.7 on a observé que ces équations sont de genre 1 dans les cas $F_{1}, F_{21}, F_{22}, F_{23}$.

$\left.F_{1}\right)$ On fait

$$
t=\frac{y-b_{1}}{y-b_{3}} \frac{b_{2}-b_{3}}{b_{2}-b_{1}}
$$

qui est la transformation de Möbius telle que

$$
b_{1} \mapsto 0, b_{2} \mapsto 1, b_{3} \mapsto \infty .
$$

Avec ce changement de variable dépendante, l'équation devient :

$$
\frac{d t}{\sqrt{t(t-1)(t-\delta)}}=\sqrt{c \sqrt[n]{B(x)}} d x
$$

où

$$
\delta:=\frac{b_{1}-b_{4}}{b_{3}-b_{4}} \frac{b_{2}-b_{3}}{b_{2}-b_{1}} \text { et } c=\left(b_{3}-b_{4}\right)\left(b_{1}-b_{2}\right) .
$$

$F_{21}$ ) D'après ce qu'on a vu dans ce chapitre, on cherche à paramétrer la courbe d'équation

$$
z^{6 n}=B(x)\left(y-b_{1}\right)^{5 n}\left(y-b_{2}\right)^{4 n}\left(y-b_{3}\right)^{3 n}
$$

comme dans IV.3.2. L'identité :

$$
\begin{aligned}
& \left(y-b_{1}\right)^{5}\left(y-b_{2}\right)^{4}\left(y-b_{3}\right)^{3} \\
& =\left(y-b_{1}\right)^{3} \frac{y-b_{2}}{y-b_{1}}\left[\left(\left(y-b_{1}\right)\left(y-b_{2}\right)\left(y-b_{3}\right)\right]^{3}\right.
\end{aligned}
$$

suggère de faire :

$$
t^{3}=\frac{y-b_{2}}{y-b_{1}}
$$


ce qui donne, en effet, une paramétrisation essentiellement du type IV.3.2. Ce changement des variables dépendantes nous ramène à l'équation à variables séparées :

$$
\frac{d t}{\sqrt{\left(b_{1}-b_{3}\right) t^{3}+\left(b_{3}-b_{2}\right)}}=\frac{1}{3} \sqrt{\left(b_{1}-b_{2}\right) \sqrt[3 n]{B(x)}} d x
$$

qui est essentiellement du type IV.4.2.

$\left.F_{22}\right)$ Considérant l'identité :

$$
\left(y-b_{1}\right)^{3}\left(y-b_{2}\right)^{3}\left(y-b_{3}\right)^{2}=\left(y-b_{1}\right)^{2}\left(y-b_{2}\right)^{4}\left(y-b_{3}\right)^{2} \frac{y-b_{1}}{y-b_{2}}
$$

on fait :

$$
t^{2}=\frac{y-b_{1}}{y-b_{2}}
$$

et l'équation devient

$$
\frac{d t}{\sqrt{\left(b_{2}-b_{3}\right) t^{3}+\left(b_{3}-b_{1}\right) t}}=\frac{1}{2} \sqrt{\left(b_{2}-b_{1}\right) \sqrt[2 n]{B(x)}} d x .
$$

$\left.F_{23}\right)$ Considérant l'identité :

$$
\left(y-b_{1}\right)^{2}\left(y-b_{2}\right)^{2}\left(y-b_{3}\right)^{2}=\left(y-b_{1}\right)^{6}\left[\frac{\left(y-b_{2}\right)\left(y-b_{3}\right)}{\left(y-b_{1}\right)^{2}}\right]^{2}
$$

on fait :

$$
\left(y-b_{2}\right)\left(y-b_{3}\right)=t^{3}\left(y-b_{1}\right)^{2} .
$$

Le discriminant de cette équation en $y$ est

$$
D=4\left(b_{1}^{2}+b_{2} b_{3}-b_{1} b_{2}-b_{2} b_{3}\right) t^{3}+\left(b_{2}-b_{3}\right)^{2} .
$$

Alors,

$$
y=\frac{2 b_{1} t^{3}-\left(b_{2}+b_{3}\right)+\sqrt{D}}{2\left(t^{3}-1\right)}
$$

et

$$
z=\sqrt[3 n]{B(x)}\left(y-b_{1}\right)^{2} t^{2} .
$$

En dérivant (IV.1) par rapport à $x$ on obtient :

$$
\left(2 y-b_{2}-b_{3}\right) y^{\prime}-2 t^{3}\left(y-b_{1}\right) y^{\prime}=3 t^{2}\left(y-b_{1}\right)^{2} t^{\prime} .
$$

En remplaçant (IV.2) dans cette équation il résulte :

$$
\sqrt{D} y^{\prime}=3 t^{2}\left(y-b_{1}\right)^{2} t^{\prime} .
$$

Finalement, puisque $y^{\prime}=z$, de (IV.3) on obtient :

$$
\frac{d t}{\sqrt{D}}=\frac{1}{3} \sqrt[3 n]{B(x)} d x
$$




\section{Chapitre V}

\section{Équations différentielles algébriques admettant des solutions transcendantes}

\section{V.1 Introduction}

Dans ce chapitre on reprend l'étude commencée au $\S 5$ du chapitre II des équations différentielles algébriques admettant des solutions avec singularités essentielles, mais d'un point de vue plus général. Considérons une équation différentielle polynomiale, comme au $\S 1$ du chapitre I. J. Malmquist a découvert que si cette équation admet une solution méromorphe sur $\mathbb{C}$ ayant une singularité essentielle à l'infini, alors elle est dans la classe FP, au sens de la définition I.4.2 (voir [26]). Ce résultat est démontré par A. Eremenko dans [11] à l'aide de la théorie de Nevanlinna. Nous démontrons ici, par des méthodes de géométrie algébrique, que, plus généralement, si l'équation admet une solution locale ayant une singularité essentielle, alors elle est dans la classe FP (théorème V.3.1; voir aussi [28]). Ces mêmes méthodes sont appliquées pour obtenir des généralisations des résultats de Yosida et de Yang ([35] et [34] respectivement); voir théorème V.4.5.

Ces résultats sont obtenus comme application du théorème V.2.1, qui établit qu'une section locale avec une singularité essentielle d'une surface fibrée $h: X \rightarrow B$ qui est tangente à un feuilletage analytique $\mathcal{F}$ de $X$ doit intersecter toute courbe algébrique horizontale non-invariante par $\mathcal{F}$ (voir V.4.5).

Si $D$ est un diviseur sur une surface analytique lisse $X$, on désigne par $\mathcal{O}_{X}(D)=\mathcal{O}(D)$ le faisceau inversible associé, c'est-à-dire, le faisceau dont l'ensemble des sections sur un ouvert $U$ de $X$ est le $\mathbb{C}[U]$-module

$$
H^{0}(U, \mathcal{O}(D)):=\left\{f \in \mathbb{C}(U): \operatorname{div} f+\left.D\right|_{U} \geq 0\right\}
$$


Observer que si $D$ et $D^{\prime}$ sont deux diviseurs sur $X$ tels que $\left.\left(D-D^{\prime}\right)\right|_{U} \geq 0$, alors on a une inclusion

$$
H^{0}\left(U, \mathcal{O}\left(D^{\prime}\right)\right) \subset H^{0}(U, \mathcal{O}(D))
$$

Dans le cas où $X$ est compacte, l'ensemble $H^{0}(X, \mathcal{O}(D))$ est un $\mathbb{C}$-espace vectoriel de dimension finie pour tout diviseur $D$. On peut donc définir une application rationnelle

$$
\phi_{D}: X-->\mathbb{P}\left(H^{0}(X, \mathcal{O}(D))\right)^{\star},
$$

où, si $V$ est un espace vectoriel, $\mathbb{P}(V)$ désigne l'espace projectif associé et $\mathbb{P}(V)^{\star}$ son dual; l'image inverse par $\phi_{D}$ d'un hyperplan dans $\mathbb{P}\left(H^{0}(X, \mathcal{O}(D))\right)^{\star}$ est donc un diviseur effectif linéairement équivalent à $D$ et $\phi_{D}$ ne dépend que de la classe d'équivalence linéaire de $D$; pour ces notions on peut consulter [31, chp. III, §1].

\section{V.2 Feuilles transcendantes des feuilletages}

Comme dans le chapitre II, $h: X \rightarrow B$ désigne une surface fibrée à fibres connexes, avec $X$ projective. Si $U \subset X$ est un ensemble ouvert de $B$, une section locale de $h$ sur $U$ est une application analytique $s: U \rightarrow X$ telle que $h(s(b))=b$ pour tout $b \in U$.

Supposons qu'il existe une application analytique surjective $\theta: X \rightarrow \mathbb{P}^{1}$ et fixons un point $b \in B$. Si $\Delta=\Delta(b)$ est un voisinage de $b$ isomorphe à un disque, on pose $\Delta^{*}:=\Delta-\{b\}, X_{b}:=h^{-1}(b), Y:=h^{-1}(\Delta)$ et $Y^{*}:=h^{-1}\left(\Delta^{*}\right)$.

L'objet de ce paragraphe est de démontrer le théorème suivant.

Théorème V.2.1. Soit $\mathcal{F}$ un feuilletage analytique à singularités isolées sur $X$. Soient $s: \Delta^{*} \rightarrow X$ une section de $h$ et $C \subset X$ une courbe irréductible non contenue dans une fibre de $h$. Supposons que

1. $C$ ne soit pas invariante par $\mathcal{F}$;

2. $s$ soit tangente $\grave{a} \mathcal{F}$;

3. $b$ soit une singularité essentielle de $\theta \circ s$.

Alors, $s\left(\Delta^{*}\right) \cap C$ n'est pas vide.

Corollaire V.2.2. Sous les hypothèses de V.2.1, $\mathcal{F}$ est génériquement transverse à $h$ (définition II.3.13).

Démonstration. Si $\mathcal{F}$ n'est pas génériquement transverse à $h$, le support du diviseur de tangence (annexe A.4) entre $\mathcal{F}$ et le feuilletage défini par la fibration $h$, doit contenir une composante irréductible $C$ qui n'est pas contenue dans une fibre de $h$. Puisque $s$ est une section de $h$, son image est transverse aux fibres régulières de celle-ci; donc $s\left(\Delta^{*}\right)$ n'intersecte pas C. 
Avant de prouver le théorème on démontre plusieurs lemmes.

Lemme V.2.3. Soit $s: \Delta^{*} \rightarrow X$ une section locale de $h$ sur $\Delta^{*}$ telle que $b$ soit une singularité essentielle de $\theta \circ s: \Delta^{*} \rightarrow \mathbb{P}^{1}$. Alors

$$
\overline{s\left(\Delta^{*}\right)} \cap X_{b}
$$

est un ensemble analytique non-vide et connexe de dimension pure 1, où $\overline{s\left(\Delta^{*}\right)}$ indique l'adhérence de $s\left(\Delta^{*}\right)$ dans $Y$.

Démonstration. Comme $s: \Delta^{*} \rightarrow Y^{*}$ est analytique et propre, $Z=s\left(\Delta^{*}\right)$ est un sous-ensemble analytique fermé irréductible de $Y^{*}$ de dimension 1 . Voyons, d'abord que $\bar{Z} \cap X_{b}$ est connexe. Soit

$$
\Delta:=\Delta_{1} \supset \Delta_{2} \supset \cdots
$$

une suite de disques de même centre que $\Delta$ et dont les rayons tendent vers 0. Alors,

$$
\bar{Z} \cap X_{b}=\bigcap_{n} \overline{s\left(\Delta_{n}-\{b\}\right)}
$$

(où la barre supérieure indique l'adhérence dans $Y$ ). Comme $\Delta_{n}-\{b\}$ est connexe, $\overline{s\left(\Delta_{n}-\{b\}\right)}$ est connexe et compacte pour $n=1,2, \ldots$ Alors $\Sigma \cap X_{b}$ est connexe.

Observons maintenant qu'il n'y a pas de point isolé dans l'ensemble $\bar{Z} \cap$ $X_{b}$. En effet, dans ce cas, puisqu'il est connexe on aurait $\bar{Z} \cap X_{b}=\{p\}$. Mais alors $s$ se prolongerait de manière continue à $\Delta$ et $b$ ne serait pas une singularité essentielle de $\theta \circ s$.

Soit maintenant $p \in \bar{Z} \cap X_{b}$ et supposons que $p$ est un point régulier de $X_{b}$; observer que $\bar{Z}$ n'est pas analytique au voisinage de $p$, car autrement il serait de dimension 1 et $p$ serait isolé dans $\bar{Z} \cap X_{b}$. Donc $p$ est un point intérieur à $\bar{Z} \cap X_{b}$ dans $X_{b}([15, \mathrm{~K}$, thm. 7$])$. On en déduit que $\bar{Z} \cap X_{b}^{\prime}$ est ouvert et fermé dans $X_{b}^{\prime}$, où $X_{b}^{\prime}$ désigne l'ensemble des points réguliers de $X_{b}$. Il en résulte que $\bar{Z} \cap X_{b}$ est la réunion de quelques composantes irréductibles de $X_{b}$, car chaque composante irréductible de $X_{b}$ est l'adhérence d'une composante connexe de $X_{b}^{\prime}$ et $X_{b}-X_{b}^{\prime}$ est un ensemble fini.

Corollaire V.2.4. L'ensemble $\overline{s\left(\Delta^{*}\right)}$ n'est contenu dans aucun sous-ensemble analytique de dimension 1 de $X$.

Démonstration. Si l'assertion était fausse, comme $s\left(\Delta^{*}\right)$ est une surface de Riemann, on aurait que $\overline{s\left(\Delta^{*}\right)}$ serait contenu dans un ensemble analytique irréductible de dimension 1. Mais l'intersection de cet ensemble avec $X_{b}$ est un ensemble fini. 
Si $\operatorname{Div}(X)$ est le $\mathbb{Z}$-module des diviseurs sur $X$, le produit d'intersection induit une forme bilinéaire symétrique $\operatorname{Div}(X) \times \operatorname{Div}(X) \rightarrow \mathbb{Z}$; on note

$$
\left(D, D^{\prime}\right) \mapsto D \cdot D^{\prime}
$$

Regardons les diviseurs dans le groupe de 2-homologie à coefficients entiers, qui est un $\mathbb{Z}$-module engendré par un nombre fini d'éléments. La forme ci-dessus passe au quotient par la relation qui consiste à avoir la même classe de Chern; la forme bilinéaire obtenue sur ce $\mathbb{Z}$-module est appelée la forme d'intersection.

Définition V.2.5. Un diviseur $D$ sur $X$ est très ample si l'application rationnelle $\phi_{D}: X--\rightarrow \mathbb{P}_{n}=\mathbb{P}\left(H^{0}(X, \mathcal{O}(D))\right)^{\star}$ est définie partout et est un plongement (c'est-à-dire un difféomorphisme sur un fermé projectif) de $X$ dans l'espace projectif $\mathbb{P}_{n}$.

Le résultat suivant, dont la preuve peut être trouvée dans $[17$, chap. V, thm. 1.10], est connu comme critère de Nakai-Moishezon : nous en donnons une version adaptée à nos besoins afin de ne pas introduire la notion de diviseur ample.

Proposition V.2.6. Soit $D$ un diviseur sur $X$. Alors, il existe un entier positif $k$ tel que $k D$ est très ample si et seulement si $D^{2}>0$ et $D \cdot C>0$ pour toute courbe irréductible $C$ de $X$.

Lemme V.2.7. Soit $C \subset X$ une courbe irréductible qui n'est pas contenue dans une fibre de $h$. Soient $A_{1}, \ldots, A_{q}$ les composantes irréductibles des fibres de $h$ qui ne rencontrent pas $C$. Soit $F$ une fibre régulière de $h$. Alors, il existe $n, m \in \mathbb{N}$ et des entiers négatifs $a_{1}, \ldots, a_{q}$ tels que le diviseur

$$
D=n F+m C+\sum_{j=1}^{q} a_{j} A_{j}
$$

vérifie $D^{2}>0$ et $D \cdot C>0$ pour toute courbe irréductible $C \subset X$.

Démonstration. Puisque $C$ rencontre toutes les fibres de $h$, le lemme de Zariski (voir [2, chap. III, lem. 8.2]) nous dit que la forme intersection définit une forme quadratique définie négative sur $\oplus_{j=1}^{q} \mathbb{Q} A_{j}$. C'est-à-dire, la matrice symétrique

$$
M=\left(c_{i j}\right), \quad c_{i j}:=A_{i} \cdot A_{j} \quad(1 \leq i, j \leq q) .
$$

est définie négative.

Comme les fibres de $h$ sont connexes, $-M$ est somme directe de matrices vérifiant les conditions de [2, cor. $\mathrm{I}(2.11)]$. 
Alors, il existe des entiers négatifs $a_{1}, \ldots, a_{q}$ tels que toutes les coordonnées du vecteur

$$
M\left(\begin{array}{l}
a_{1} \\
\vdots \\
a_{q}
\end{array}\right)
$$

sont positives ; ceci signifie

$$
\left(\sum_{j=1}^{q} a_{j} A_{j}\right) \cdot A_{i}>0, i=1, \ldots, q
$$

posons $D:=n F+m C+\sum_{j=1}^{q} a_{j} A_{j}$, où $n, m \in \mathbb{N}$.

Tout d'abord

$$
D \cdot F=m C \cdot F>0 \forall n, m \in \mathbb{N} \text {. }
$$

Ensuite,

$$
D \cdot A_{i}=\left(\sum_{j=1}^{q} a_{j} A_{j}\right) \cdot A_{i}>0, \forall n, m \in \mathbb{N}, i=1, \ldots, q .
$$

Soit $A$ une composante irréductible d'une fibre singulière de $h$ telle que $A \cap C$ est non vide. Alors,

$$
D \cdot A=m C \cdot A+\sum_{j=1}^{q} a_{j} A_{j} \cdot A
$$

Comme il n'y a qu'un nombre fini de telles courbes $A$ et $C \cdot A \geq 1$, on peut trouver $m \in \mathbb{N}$ tel que

$$
D \cdot A>0 \forall n \in \mathbb{N}
$$

fixons un tel $m$. Soit $S$ une courbe irréductible qui n'est pas contenue dans une fibre de $h$. Alors, comme $a_{j}<0$ pour tout $j$ et $A_{j} \cdot S \leq F \cdot S$, on a

$$
\begin{aligned}
D \cdot S & =n F \cdot S+m C \cdot S+\sum_{j=1}^{q} a_{j} A_{j} \cdot S \\
& \geq n F \cdot S+m C \cdot S+\left(\sum_{j=1}^{q} a_{j}\right) F \cdot S \\
& =\left(n+\sum_{j=1}^{q} a_{j}\right) F \cdot S+m C \cdot S .
\end{aligned}
$$

Comme $F \cdot S>0$ et $C \cdot S \geq 0$ si $C \neq S$, il existe $n_{0} \in \mathbb{N}$ tel que $D \cdot S>0$ pour toute courbe irréductible qui n'est pas contenue dans une fibre de $h$ 
et tout $n>n_{0}$. Finalement,

$$
D^{2}=m^{2} C^{2}+\left(\sum_{j=1}^{q} a_{j} A_{j}\right)^{2}+2 m n C \cdot F .
$$

Puisque $C \cdot F>0$ on a que $D^{2}>0$, si $n \in \mathbb{N}$ est assez grand.

Si $D$ est un diviseur sur $X$, on note $\operatorname{Supp}(D)$ le support de $D$.

Sous les hypothèses du lemme V.2.7, on a

Lemme V.2.8. Il existe $f \in \mathbb{C}(X)$ telle que

1. $C \subset \operatorname{Supp}\left(\operatorname{div}_{\infty} f\right) \subset C \cup F$;

2. pour chaque $j=1, \ldots, q$, la fonction $f$ est holomorphe au voisinage de chaque point de $A_{j}$ et $\left.f\right|_{A_{j}}=0$.

Démonstration. Soient $n, m \in \mathbb{N}$ et $a_{j}<0(j=1, \ldots, q)$ des entiers tels que

$$
D=n F+m C+\sum_{j=1}^{q} a_{j} A_{j}
$$

vérifie les conditions du lemme V.2.7. Considérons le diviseur

$$
D^{\prime}:=n F+\sum_{j=1}^{q} a_{j} A_{j} .
$$

Alors

$$
\left(D^{\prime}\right)^{2}=\left(\sum_{j=1}^{q} a_{j} A_{j}\right)^{2} \leq 0
$$

par le lemme de Zariski; en particulier, si $k \in \mathbb{Z}$ alors $k D^{\prime}$ n'est pas très ample (V.2.6).

D'après la proposition V.2.6 il existe $k \in \mathbb{N}$ tel que $k D$ est très ample. Comme $k D^{\prime} \leq k D$ et $k D^{\prime}$ n'est pas très ample, on doit avoir une inclusion $\underline{\text { stricte }}$

$$
H^{0}\left(X, \mathcal{O}\left(k D^{\prime}\right)\right) \subset H^{0}(X, \mathcal{O}(k D)) .
$$

Alors, il existe $f \in \mathbb{C}(X)$ telle que

$$
\operatorname{div} f+k D \geq 0 \text { mais } \operatorname{div} f+k D^{\prime} \nsupseteq 0 .
$$

On en déduit, respectivement,

$$
\operatorname{Supp}\left(\operatorname{div}_{\infty} f\right) \subset F \cup C \text { et } C \subset \operatorname{Supp}\left(\operatorname{div}_{\infty} f\right) .
$$

De la première inclusion résulte que $f$ est holomorphe au voisinage de chaque point de $A_{j}(j=1, \ldots, q)$. Comme $a_{j}<0$ et $\operatorname{div} f+k D \geq 0$ on obtient que $\left.f\right|_{A_{j}}=0$ d'où suit l'assertion. 
Observation V.2.9. Soient $V$ une surface de Riemann et $W$ une surface analytique. Soit $\varphi: V \rightarrow W$ analytique telle que $\varphi(V)$ n'est contenu dans aucun sous-ensemble analytique de dimension 1 de $W$. Alors $f \circ \varphi$ est définie pour toute $f \in \mathbb{C}(W)$ et l'application $f \mapsto f \circ \varphi$ est un homomorphisme injectif $\mathbb{C}(W) \rightarrow \mathbb{C}(V)$.

Lemme V.2.10. Soient $C \subset X$ une courbe irréductible non contenue dans une fibre de $h$ et $s: \Delta^{*}: \rightarrow X$ une section locale de $h$. Si $b \in B$ est une singularité essentielle de $\theta \circ s: \Delta^{*} \rightarrow \mathbb{P}^{1}$, il existe une composante irréductible $A$ de $X_{b}$ telle que

1. $A \cap C \neq \emptyset$;

2. $\overline{s\left(\Delta^{*}\right)} \supset A$.

Démonstration. Notons $B_{1}, \ldots, B_{r}$ les composantes irréductibles de $X_{b}$ qui ne rencontrent pas $C$. Si $r=0$, le lemme suit immédiatement du lemme V.2.3 et du fait que $X_{b} \cap C$ n'est pas vide. Si $r>0$, supposons, par l'absurde, que le lemme soit faux. D'après le lemme V.2.3 on doit avoir

$$
\overline{s\left(\Delta^{*}\right)} \cap X_{b} \subset \bigcup_{j=1}^{r} B_{j} .
$$

Soit $f \in \mathbb{C}(X)$ comme dans le lemme V.2.8.

D'après la condition (1) de ce lemme, $f \notin \mathbb{C}(B)$ (on considère $\mathbb{C}(B) \subset$ $\mathbb{C}(X)$ par composition avec $h)$

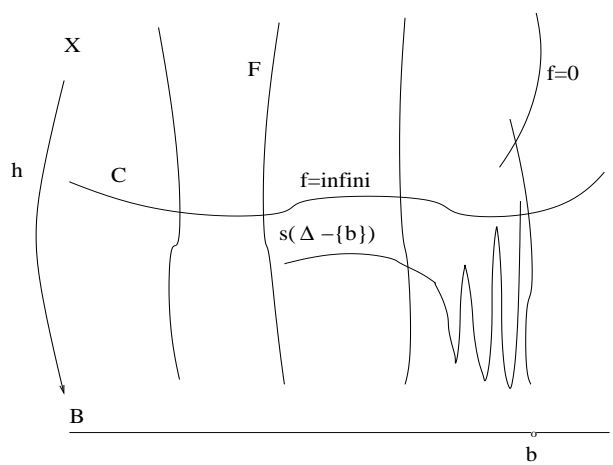

D'autre part, $f$ est holomorphe au voisinage de chaque point de $B_{j}$ et $\left.f\right|_{B_{j}}=0$. Alors, $f \circ s: \Delta^{*} \rightarrow \mathbb{P}^{1}$ se prolonge en une fonction analytique sur $\Delta$, puisque, d'après (V.1)

$$
\lim _{y \rightarrow b} f(s(y))=0 .
$$


Quitte à rétrécir $\Delta$, on peut supposer

$$
f \circ s(\Delta) \subset \mathbb{C} \text {. }
$$

Le fait que $b$ soit une singularité essentielle de $\theta \circ s$ entraîne aussi que $\theta \notin \mathbb{C}(B)$. Puisque le degré de transcendance de $\mathbb{C}(X)$ sur $\mathbb{C}(B)$ est 1 , il existe

$$
P(T, Z) \in \mathbb{C}(B)[T, Z]
$$

non-nul tel que $P(f, \theta)=0$. Posons

$$
P(T, Z)=\sum_{j=0}^{N} a_{j}(T) Z^{j}, a_{j}(T) \in \mathbb{C}(B)[T], a_{N}(T) \neq 0 .
$$

Comme $\mathbb{C}(B) \subset \mathbb{C}(X)$ est une extension transcendante pure, étant donné que les fibres de $h$ sont connexes, alors $a_{j}(f)=0$ implique $a_{j}(T)=0$. Par ailleurs, $\overline{s\left(\Delta^{*}\right)}$ n'est pas contenu dans un ensemble analytique de dimension 1 d'après le corollaire V.2.4. Donc, $a_{j}(f) \circ s=0$ implique $a_{j}(f)=0$. On en déduit que $a_{N}(f) \circ s=a_{N}(f \circ s) \neq 0$. De l'équation $P(f, \theta)=0$ on déduit $P(f \circ s, \theta \circ s)=0$. Donc

$$
\sum_{j=1}^{N} a_{j}(f \circ s)(\theta \circ s)^{j}=0 .
$$

Comme $f \circ s$ est holomorphe sur $\Delta$, la fonction $a_{j}(f \circ s)$ est méromorphe sur $\Delta$. Puisque $a_{N}(f \circ s) \neq 0$, on obtient que $\theta \circ s$ est une fonction algébrique sur $\Delta$. Alors, elle n'a pas de singularité essentielle : contradiction.

Soit $\mathcal{F}$ un feuilletage analytique à singularités isolées de $X$.

Si $\pi: \widetilde{X} \rightarrow X$ est un morphisme birationnel, on sait qu'il existe un sous-ensemble fini $S \subset X$ tel que

$$
\left.\pi\right|_{\widetilde{X}-\pi^{-1}(S)}: \widetilde{X}-\pi^{-1}(S) \longrightarrow X-S
$$

est un isomorphisme (voir [17, chap. V, Ex. 5.1.1 et prop. 5.3]).

On démontre qu'il existe un feuilletage à singularités isolées unique $\widetilde{\mathcal{F}}$ de $\widetilde{X}$ tel que $\left.\pi\right|_{\widetilde{X}-\pi^{-1}(S)}$ identifie $\left.\widetilde{\mathcal{F}}\right|_{\widetilde{X}-\pi^{-1}(S)}$ avec $\left.\mathcal{F}\right|_{X-S}$. En effet, $\pi$ est une suite finie d'éclatements successifs de points et alors l'affirmation découle de $[6$, chap. I, $\S 2]$. On dit que $\widetilde{\mathcal{F}}$ est le relèvement de $\mathcal{F}$ à $\widetilde{X}$.

Si $C \subset X$ est une courbe irréductible on définit le transformé strict (inverse) $\widetilde{C}$ de $C$ par $\pi$ comme l'adhérence (Zariski) de $\pi^{-1}(C-S)$.

Soit $C \subset X$ une courbe irréductible qui n'est pas invariante par $\mathcal{F}$. Dans [6, chap. II, §2] on associe à $C$ et $\mathcal{F}$ un entier non-négatif $\operatorname{tang}(\mathcal{F}, C)$ qui mesure l'ordre de tangence entre $\mathcal{F}$ et $C$. Soit $p \in C$ et supposons que $\pi: \widetilde{X} \rightarrow X$ est l'éclatement de $X$ dans $p$. Il résulte de [6, chap. II, 
prop. 2 et ex. 1] que si $C$ est lisse et $p$ est un point singulier de $\mathcal{F}$ alors $\operatorname{tang}(\widetilde{\mathcal{F}}, \widetilde{C})<\operatorname{tang}(\mathcal{F}, C)$. On sait qu'on peut rendre $C$ lisse au moyen d'un nombre fini d'éclatements ([17, chap. V, thm. 3.9]). Alors, on a démontré le lemme suivant.

Lemme V.2.11. Il existe un morphisme birationnel $\pi: \widetilde{X} \rightarrow X$ tel que $\widetilde{C}$ ne contient pas de point singulier de $\widetilde{\mathcal{F}}$.

Preuve du théorème V.2.1 Tout d'abord, par le lemme V.2.11 on peut supposer que $C$ ne contient pas de point singulier de $\mathcal{F}$.

En effet, en prenant $\Delta$ suffisamment petit, on peut supposer que la section $s$ se relève en $\tilde{s}: \Delta^{*} \rightarrow \widetilde{X}$ (puisque $\pi^{-1}$ est holomorphe en-dehors d'un ensemble fini); notons $\tilde{\theta}:=\theta \circ \pi$. Maintenant $\tilde{\theta} \circ \tilde{s}=\theta \circ \pi \circ \tilde{s}$ et $b$ est une singularité essentielle de $\tilde{\theta} \circ \tilde{s}$. Il suffit, donc, de substituer $h, s, \theta$ et $C$ par $\tilde{h}:=h \circ \pi, \tilde{s}, \tilde{\theta}$ et $\tilde{C}$ respectivement. On traite maintenant le cas où $C$ ne contient pas de singularité de $\mathcal{F}$. Soit $A$ la composante irréductible de $X_{b}$ donnée par le lemme V.2.10. Pour tout voisinage connexe $V_{b} \subset \Delta$ de $b$, on sait que $s\left(V_{b}^{*}\right)$ est une feuille de la restriction de $\mathcal{F}$ à $h^{-1}\left(V_{b}^{*}\right)$; ici on a posé $V_{b}^{*}:=V_{b}-\{b\}$. Du lemme II.5.3 suit que $A$ est invariante par $\mathcal{F}$. Prenons $p \in C \cap A$. Puisque $p$ n'est pas singulier pour $\mathcal{F}$, la structure produit de $\mathcal{F}$ au voisinage de $p$ (proposition A.1.7) et le fait que $p \in \overline{s\left(\Delta^{*}\right)}$ nous montre tout de suite que $s\left(\Delta^{*}\right) \cap C$ n'est pas vide, d'où suit le théorème.

\section{V.3 Classe FP et solutions transcendantes}

Considérons l'équation différentielle polynomiale

$$
F\left(x, y, y^{\prime}\right)=0,
$$

où

$F(x, y, z)=c_{0}(x, y) z^{m}+c_{1}(x, y) z^{m-1}+\cdots+c_{m}(x, y), m \geq 1, c_{0}(x, y) \neq 0$

est un polynôme irréductible.

Rappelons (voir définition II.5.1) qu'un point $a \in \mathbb{C}$ est une singularité essentielle de (V.2) s'il existe un disque ouvert $D \subset \mathbb{C}$ de centre 0 et des fonctions

$$
\alpha: D \rightarrow \mathbb{C}, \beta: D^{*} \rightarrow \mathbb{C}\left(D^{*}:=D-\{0\}\right)
$$

telles que $\alpha(0)=a, \alpha$ est holomorphe et non-constante, $\beta$ est méromorphe, 0 est une singularité essentielle de $\beta$ et

$$
F\left(\alpha(t), \beta(t), \frac{\beta^{\prime}(t)}{\alpha^{\prime}(t)}\right)=0
$$

en tout $t \in D^{*}$ qui ne soit ni un pôle de $\beta$ ni un zéro de $\alpha^{\prime}$. On dira que $\infty$ est une singularité essentielle de (V.2) si 0 l'est pour l'équation 
obtenue de celle-ci par le changement de variable $x_{1}=1 / x$; observons que ce changement de variables laisse invariante la classe FP des équations polynomiales.

On garde les notations du $\S 3$ du chapitre II. En particulier le diagramme commutatif de II.3 donne lieu au diagramme commutatif

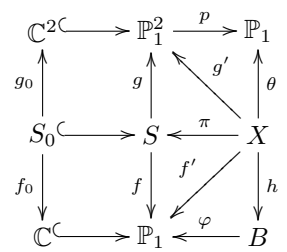

où $p: \mathbb{P}_{1}^{2} \rightarrow \mathbb{P}_{1}$ est la projection sur le deuxième facteur, $\theta:=p \circ g^{\prime}$ et $\varphi$ est un revêtement ramifié tel que $f$ se factorise comme $\varphi \circ h$ avec $h$ à fibres connexes.

Dans la preuve du théorème suivant on utilisera la propriété suivante des désingularisations : si $\pi: X \rightarrow S$ est une désingularisation de la surface $S$, $U \subset \mathbb{C}$ est un ouvert connexe et $\sigma: U \rightarrow S$ est holomorphe et telle que $\sigma(U) \not \subset \operatorname{Sing}(S)$ alors

$$
\left.\left(\pi^{-1} \circ \sigma\right)\right|_{U \backslash \sigma^{-1}(\operatorname{Sing}(S))}
$$

se prolonge analytiquement à $U$.

Théorème V.3.1. Supposons que (V.2) possède une singularité essentielle en un point $a \in \mathbb{P}_{1}$. Alors, (V.2) est dans la classe FP.

Démonstration. Soient $h: X \rightarrow B$ et $\mathcal{F}$ la surface fibrée et le feuilletage de $X$ associés à l'équation (V.2). Par le théorème II.3.15, il suffit de montrer que $\mathcal{F}$ est génériquement transverse à $h$. On peut supposer $a \in \mathbb{C}$.

Par hypothèse il existe une solution locale de (V.2) ayant une singularité essentielle en $a$. Quitte à faire un changement de variable indépendante de la forme

$$
x=a+t^{M}
$$

on peut supposer que la solution locale est uniforme; soient $U^{*}$ un voisinage épointé de $a$ et $u: U^{*} \rightarrow \mathbb{P}_{1}$ cette solution locale (uniformisée), qui possède une singularité essentielle en $a$.

La fonction $u$ définit une application analytique $v: U^{*} \rightarrow S$ telle que $f \circ v=I d$ et $p \circ g \circ v=u$, où $v(x)=\left(x, u(x), u^{\prime}(x)\right)$.

Par ailleurs, notons $V \subset S_{0} \subset S$ l'ouvert de Zariski (dense) où $g^{\prime}$ devient un isomorphisme local : il est constitué de points réguliers de $S$ (voir chap. II, $\S 3) ; v\left(U^{*}\right)$ n'est pas contenu dans $S-V$, car autrement le graphe de $u$ dans $U^{*} \times \mathbb{P}_{1}$ serait contenu dans le sous-ensemble analytique $g(S-V)$ 
de $\mathbb{P}_{1}^{2}$ contredisant l'existence de singularité essentielle (corollaire V.2.4). Donc $L:=U^{*}-v^{-1}(V)$ est discret et $\pi^{-1} \circ\left(\left.v\right|_{v^{-1}(V)}\right)$ se prolonge en une application analytique $w: U^{*} \rightarrow X$ telle que l'on a :

(i) $\quad w\left(U^{*}\right)$ est invariante par $\mathcal{F}$

(ii) $f^{\prime} \circ w=f \circ v=I d$

(iii) $\theta \circ w=p \circ g^{\prime} \circ \pi \circ w=p \circ g \circ v=u$;

en particulier $w$ est une section locale de la fibration $f^{\prime}$.

Comme on sait par le lemme V.2.3 $\overline{w\left(U^{*}\right)} \cap\left(f^{\prime}\right)^{-1}(a)$ est connexe (observer qu'on n'a pas utilisé l'hypothèse de fibres connexes dans la preuve de ce lemme) ; prenons $b \in B$ avec $\varphi(b)=a$ de sorte que $h^{-1}(b)$ soit la composante connexe de $\left(f^{\prime}\right)^{-1}(a)$ qui contient $\overline{w\left(U^{*}\right)} \cap\left(f^{\prime}\right)^{-1}(a)$. L'application $s: \Delta^{*} \rightarrow X$ définie sur un voisinage épointé $\Delta^{*}$ de $b$ par

$$
s:=w \circ\left(\left.\varphi\right|_{\Delta^{*}}\right)
$$

est, par construction, une section (locale) de $h$, invariante par $\mathcal{F}$, telle que $\theta \circ s$ a une singularité essentielle en $b \in B$.

Il suffit alors d'appliquer le corollaire V.2.2.

On sait qu'une équation différentielle algébrique de genre $>1$ qui est dans la classe FP possède une intégrale première méromorphe (voir III.6.4). On en déduit immédiatement le résultat suivant.

Corollaire V.3.2. Supposons que l'équation (V.2) soit de genre $>1$. Alors, elle ne possède pas de singularité essentielle.

Pour finir on montre que le théorème V.2.3 fournit, comme cas particulier, les théorèmes de Yosida ([35]) et Wittich ([33]) qui généralisent, à leur tour, le théorème de Malmquist ([24]).

Corollaire V.3.3. Si l'équation (V.2) possède une singularité essentielle, alors

$$
\operatorname{deg}_{y} c_{k} \leq 2 k, k=0, \ldots, m .
$$

Démonstration. Voir théorème I.4.13

Théorème V.3.4. (K. Yosida) Soit $R(x, y)$ une fonction rationnelle. Supposons que l'équation différentielle

$$
\left(y^{\prime}\right)^{m}=R(x, y)
$$

admette une solution méromorphe sur $\mathbb{C}$ qui n'est pas rationnelle. Alors $R(x, y)$ est polynomiale en $y$ et $\operatorname{deg}_{y} R(x, y) \leq 2 m$. 
Démonstration. Il suffit d'écrire $R(x, y)=A(x, y) / B(x, y)$ avec $A, B \in$ $\mathbb{C}[x, y]$ et d'appliquer le corollaire V.3.3 à $F(x, y, z):=B(x, y) z^{m}-A(x, y)$; le cas où $F$ est réductible se réduit au cas où il est irréductible (voir [23, chap VIII, $\S 9$, thm.16]).

Observation V.3.5. Le théorème de Malmquist est le théorème V.3.4 pour $m=1$.

Exemple V.3.6. Considérons l'équation

$$
2 y y^{\prime}-y^{2}+1=0 .
$$

Elle possède la solution

$$
y=\sqrt{e^{x}-1}
$$

observons que cette équation n'est pas dans la classe FP.

\section{V.4 Quelques applications}

Reprenons l'équation différentielle polynomiale

$$
F\left(x, y, y^{\prime}\right)=0
$$

où

$$
F(x, y, z):=\sum_{k=0}^{m} c_{k}(x, y) z^{m-k}\left(m \geq 1, c_{0} \neq 0\right)
$$

est un polynôme irréductible à coefficients complexes.

Définition V.4.1. Une solution transcendante uniforme de (V.3) est une

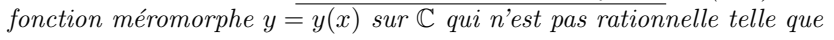

$$
F\left(x, y(x), y^{\prime}(x)\right)=0 .
$$

L'existence d'une solution transcendante uniforme implique que $\infty$ est une singularité essentielle de l'équation. Alors le théorème V.3.1 implique le résultat suivant :

Proposition V.4.2. Si V.3 admet une solution transcendante uniforme alors elle est dans la classe FP.

Considérons le cas particulier d'une équation linéaire de la forme

$$
a(x) y^{\prime}+b(x) y+c(x)=0, a, b, c \in \mathbb{C}[x], a \neq 0 .
$$

Puisque l'ordre d'un pôle de $y^{\prime}$ est un de plus que l'ordre de pôle de $y$ au même point, on voit que l'ensemble des pôles d'une solution est contenu 
dans l'ensemble des zéros de $a(x)$; en particulier, cet ensemble est fini. De même, si l'équation est homogène (c'est-à-dire, $c(x)=0$ ), toute solution transcendante uniforme n'a qu'un nombre fini de zéros.

Il résulte des résultats de C. C. Yang [34] que si une équation du type

$$
y^{\prime}=R(x, y)
$$

où $R$ est une fonction rationnelle à coefficients complexes, admet une solution transcendante uniforme n'ayant qu'un nombre fini de pôles, alors cette équation est linéaire; si de plus, la solution n'a qu'un nombre fini de zéros, alors cette équation est linéaire homogène.

La première de ces assertions est fausse pour les équations polynomiales comme on le voit dans l'exemple suivant. Cependant, la deuxième reste valable (théorème V.4.10).

Exemple V.4.3. Soit $y=u(x)$ une solution holomorphe sur $\mathbb{C}$ de l'équation

$$
y^{\prime}=2 x y+x^{2} .
$$

On voit tout de suite que $u$ n'est pas un polynôme. Alors, $y=u(x)^{2}$ est une solution transcendante uniforme sans pôles de l'équation :

$$
\left(y^{\prime}\right)^{2}-8 x y y^{\prime}+16 x^{2} y^{2}-4 x^{4} y=0
$$

(observons que $z^{2}-8 x y z+16 x^{2} y^{2}-4 x^{4} y$ est irréductible même comme polynôme en $y, z$ sur le corps de fonctions rationnelles sur $x$ ).

J. Malmquist démontre que si l'équation V.4 admet une solution transcendante uniforme alors elle est une équation de Riccati. Le résultat de C.C. Yang cité plus haut équivaut à dire que si cette solution n'a qu'un nombre fini de pôles alors $y=\infty$ est aussi une "solution" de l'équation de Riccati.

Nous prouvons, plus généralement, que si une solution transcendante uniforme de V.3 ne rencontre une courbe algébrique donnée qu'en un nombre fini de points, alors cette courbe est une solution algébrique (V.4.5).

Définition V.4.4. Considérons l'équation différentielle polynomiale

$$
G\left(x, t, t^{\prime}\right)=0 .
$$

On dira que (V.3) se ramène rationnellement à (V.6) s'il existe une fonc-

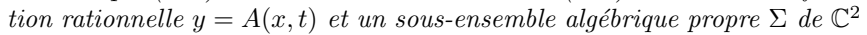
tels que:

a) si $t=t(x)$ est une solution de (V.6) méromorphe sur un ouvert connexe $U$ de $\mathbb{C}$ qui n'annule pas le dénominateur de $A$, alors $y=A(x, t(x))$ est une solution de (V.3);

b) si $y=y(x)$ est une solution de (V.3) méromorphe sur un ouvert connexe $U$ de $\mathbb{C}$ telle que l'ensemble

$$
\{x \in \mathbb{C}:(x, y(x)) \notin \Sigma\}
$$


n'est pas vide, alors il existe une solution $t=t(x)$ de (V.6) méromorphe sur $U$ telle que $y(x)=A(x, t(x))$.

On a les deux résultats suivants dont la preuve sera donnée tout de suite après le lemme V.4.9.

Théorème V.4.5. Supposons que l'équation (V.3) admette une solution transcendante uniforme $y=u(x)$ et qu'il existe un polynôme irréductible à coefficients complexes $P(x, y)$, qui dépend effectivement de $y$, tel que $P(x, u(x))$ n'a qu'un nombre fini de zéros. Alors

a) pour tout $a \in \mathbb{C}$ générique la courbe $F(a, y, z)=0$ est rationnelle et irréductible;

b) la courbe d'équation $P(x, y)=0$ est une solution algébrique de (V.3);

c) il existe une, deux ou une infinité de solutions transcendantes uniformes de (V.3);

d) dans le premier et troisième cas de (c) l'équation (V.3) se ramène rationnellement à une équation linéaire à coefficients rationnels;

e) dans le deuxième cas de (c) l'équation (V.3) se ramène rationnellement $\grave{a}$ une équation de Riccati à coefficients rationnels.

Corollaire V.4.6. Supposons que l'équation (V.3) admette une solution transcendante uniforme n'ayant qu'un nombre fini de pôles. Alors

$$
\operatorname{deg}_{y} c_{k} \leq 2 k \text { si } 0 \leq k \leq m-1 \text { et } \operatorname{deg}_{y} c_{m}<2 m
$$

(pour $m=1$ c'est le résultat de C.C. Yang). De plus, les affirmations $(a),(c),(d)$ et $(e)$ du théorème restent valables.

Observation V.4.7. On vérifie facilement qu'une équation linéaire à coefficients rationnels n'admet aucune solution transcendante uniforme ou bien elle en admet une ou une infinité. Les équations

$$
y^{\prime}=\frac{1}{2 x} y, y^{\prime}=\left(1+\frac{1}{2 x}\right) y+1, y^{\prime}=y
$$

constituent des exemples de ces trois cas respectivement; la deuxième équation possède la solution transcendante uniforme

$$
y=e^{x} \sum_{n=0}^{\infty} \frac{(-1)^{n}}{n !\left(n+\frac{1}{2}\right)} x^{n+1} .
$$

Exemple V.4.8. 1) L'équation (V.5) se ramène rationnellement à l'équation linéaire $t^{\prime}=2 x t+x^{2}$ en faisant $y=t^{2}$.

2) L'équation

$$
x^{2}\left(y^{\prime}\right)^{2}+2 x y y^{\prime}+y^{2}-4 x^{2} y-4 x^{3} y^{2}=0
$$


se ramène rationnellement à l'équation de Riccati

$$
t^{\prime}=t^{2}+\frac{1}{2 x} t-x
$$

au moyen du changement de variable dépendante

$$
y=\frac{1}{t^{2}-x} .
$$

L'équation (V.8) admet les solutions transcendantes uniformes :

$$
t=f(x)=\frac{1+\exp \left(\frac{4}{3} x^{3 / 2}\right)}{1-\exp \left(\frac{4}{3} x^{3 / 2}\right)} \sqrt{x} \text { et } t=h(x)=x / f(x) .
$$

Rappelons que trois solutions différentes d'une équation de Riccati déterminent rationnellement toutes les autres. Alors, s'il existait une autre solution méromorphe sur $\mathbb{C}$ de (V.8), toutes les solutions serait uniformes, ce qui est absurde puisque $t=\sqrt{x}$ est une solution. Il en résulte que (V.7) admet exactement deux solutions transcendantes uniformes :

$$
y=\frac{1}{f(x)^{2}-x} \text { et } y=\frac{1}{h(x)^{2}-x}
$$

qui n'ont pas de pôle en dehors de 0 . Si on fait le changement de variable dépendante $y=1 / y_{1}$ dans (V.7) on obtient une équation différentielle polynomiale qui vérifie l'hypothèse du théorème V.4.5 avec

$$
P(x, y)=y \text { et } u(x)=f(x)^{2}-x
$$

et qui admet exactement deux solutions transcendantes uniformes.

3) Soient $p, q \in \mathbb{C}$ tels que $y^{3}+p y+q$ est séparable. L'équation

$$
\left(y^{\prime}\right)^{2}=y^{3}+p y+q
$$

a pour solution la fonction de Weierstrass $y=\wp(x)$ correspondant à la courbe elliptique $z^{2}=y^{3}+p y+q$. Alors, d'après la partie (a) du théorème V.4.5, la fonction $P(x, \wp(x))$ admet une infinité de zéros pour tout polynôme $P(x, y)$ à coefficients complexes qui dépend effectivement de $y$.

4) Considérons la fonction transcendante uniforme

$$
y=f(x)=\left(e^{x}-x\right) /\left(x e^{x}-1\right) .
$$

Affirmation. Si $P(x, y)$ est un polynôme irréductible, à coefficients complexes et dépendant effectivement de $y$ tel que $P(x, f(x))=0$ n'a qu'un nombre fini de zéros alors

$$
P(x, y)=c(x-y), c \in \mathbb{C}, c \neq 0 .
$$


Preuve. $y=f(x)$ est une solution de l'équation de Riccati

$$
\left(1-x^{2}\right) y^{\prime}=-(1+x) y^{2}+\left(1+x^{2}\right) y-x+1 .
$$

On vérifie que $y=x$ est une solution de cette équation, en accord avec le théorème V.4.5b. Le changement de variable $y_{1}=1 /(y-x)$ nous ramène à une équation linéaire dont $1 /(f(x)-x)$ est une solution particulière. Il en résulte que la solution générale de l'équation de Riccati est :

$$
y=x, y=x+\frac{e^{x}\left(1-x^{2}\right)^{2}}{\left(x e^{x}-1\right)\left(1-x^{2}\right)+\lambda\left(1-2 x-x^{2}\right)}, \lambda \in \mathbb{C} .
$$

On en déduit que la seule solution algébrique est $y=x$. L'affirmation découle, alors, du théorème V.4.5b.

Lemme V.4.9. Soit $k: X \rightarrow A$ une application analytique surjective où $X$ est une surface analytique compacte, lisse et connexe et $A$ est une surface de Riemann. Soit $A_{0}$ un ouvert de $A$ tel que $A-A_{0}$ est un ensemble fini. Supposons qu'il existe $s: A_{0} \rightarrow X$ analytique telle que $k(s(y))=y$ pour tout $y \in A_{0}$. Alors, les fibres de $k$ sont connexes.

Démonstration. Le théorème de factorisation de Stein ([17, chap. III, cor. 11.5]) nous dit qu'il existe une surface de Riemann compacte $B$ et des applications holomorphes surjectives $h: X \rightarrow B$ et $\varphi: B \rightarrow A$ telles que le diagramme suivant

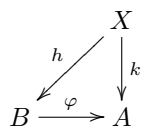

commute et que les fibres de $h$ sont connexes; soit $t:=h \circ s: A_{0} \rightarrow B$. Alors $\varphi(t(y))=y$ pour tout $y \in A_{0}$. Comme les points de $A-A_{0}$ sont isolés et les fibres de $\varphi$ sont discrètes, il est facile de voir que $t$ se prolonge en une application continue, donc holomorphe, $u: A \rightarrow B$. Alors $\varphi \circ u=I d$ d'où suit que $\varphi$ est un isomorphisme.

Preuve du théorème V.4.5.

On reprend les notations du $\S 3$.

L'application

$$
x \mapsto\left(x, u(x), u^{\prime}(x)\right)
$$

est une section holomorphe sur $\mathbb{C}$ de $f$. Le fait que $\infty$ soit une singularité essentielle de $u$ implique que l'image de cette section n'est pas contenue dans l'ensemble des singularités de $S$. Donc, cette section se relève à une section holomorphe $s$ de $f^{\prime}$ sur $\mathbb{C}$ (voir le commentaire précédant au théorème V.3.1). D'après le lemme V.4.9, les fibres de $f^{\prime}$ sont connexes. 
Soit $C \subset X$ une courbe algébrique fermée irréductible telle que $g(C)$ contienne la courbe de $\mathbb{C}^{2} \subset \mathbb{P}_{1} \times \mathbb{P}_{1}$ définie par l'équation $P(x, y)=0$. Alors $\theta \circ s$ possède une singularité essentielle en $\infty$ et $s\left(U_{\infty}\right) \cap C$ est vide si $U_{\infty}$ est un voisinage convenable de $\infty$ dans $\mathbb{P}_{1}$, par hypothèse. On est donc dans la situation du théorème $\mathrm{V} .2 .1$. Il en résulte que $C$ est invariante par $\mathcal{F}$, ce qui entraîne tout de suite (b) (voir chap. II, §3).

Par la proposition V.4.2, l'équation (V.3) est dans la classe FP. De plus, le genre $g$ de cette équation (chap. II, §3) est $g=0$ ou $g=1$ par le corollaire V.3.2. Supposons $g=1$. Dans ce cas, l'existence de la courbe $C$, qui est invariante par $\mathcal{F}$ et n'est pas contenue dans une fibre de $f$, implique l'existence d'une intégrale première rationnelle de (V.3) (voir IV.4.4 et IV.3.3e). Cela est absurde, car par hypothèse cette équation admet une solution transcendante. On conclut que $g=0$. Comme les fibres de $f^{\prime}$ sont connexes, ceci implique (a) (voir II.3.4 et II.3.5). De plus, on a que (V.3) se ramène rationnellement à une équation de Riccati :

$$
t^{\prime}=a(x) t^{2}+b(x) t+c(x)
$$

à coefficients rationnels ( $\$ 4$ et $\S 5$ du chap. III).

La section $s$ et la courbe $C$, invariants par $\mathcal{F}$, donnent lieu respectivement, à une solution transcendante uniforme $t=k(x)$ et à une solution algébrique $t=h(x)$ de (V.9) (éventuellement $h(x)=\infty$, auquel cas (V.9) est linéaire).

Si $h$ est une fonction rationnelle, (V.9) se ramène à une équation linéaire à coefficients rationnels. Dans ce cas (V.3) admet une solution transcendante uniforme ou en admet une infinité (observation V.4.7). Pour compléter la preuve du théorème il suffit de montrer que si $h$ n'est pas une fonction rationnelle, alors (V.9) admet exactement deux solutions transcendantes uniformes.

Puisque trois solutions différentes de (V.9) déterminent rationnellement toutes les autres, si cette équation admettait trois solutions uniformes, toutes les autres le seraient aussi, ce qui est impossible puisque $h$, par hypothèse, ne l'est pas. De même, si le degré de $h$ était $>2$, alors toutes les solutions seraient algébriques, ce qui contredit l'existence de $k$ (III.5.7).

Comme le degré de $h$ est 2 on peut supposer, par simple changement de variable, que $h^{2}$ est une fonction rationnelle, disons $h^{2}(x)=r(x)$. Alors

$$
h^{\prime}(x)=a(x) r(x)+b(x) h(x)+c(x) .
$$

D'autre part

$$
h^{\prime}(x)=\frac{r^{\prime}(x)}{2 h(x)}
$$

d'où

$$
r^{\prime}(x)=2 a(x) r(x) h(x)+2 b(x) r(x)+2 c(x) h(x) .
$$

Puisque $h(x)$ n'est pas rationnelle, on en déduit

$$
a(x) r(x)+c(x)=0 \text { et } r^{\prime}(x)=2 b(x) r(x) .
$$


C'est-à-dire

$$
a(x) h^{2}(x)+c(x)=0 \text { et } h^{\prime}(x)=b(x) h(x) ;
$$

ceci montre que $-h(x)$ est aussi solution de (V.9)

Comme la conjuguée algébrique d'une solution est aussi une solution, on a que $-h$ est une solution de (V.9). On en déduit que

$$
t=\frac{h^{2}(x)}{k(x)}
$$

est une solution de (V.9). Cette solution est transcendante, uniforme et différente de $t=k(x)$.

Preuve du corollaire V.4.6. D'après le corollaire V.3.3 on a

$$
\operatorname{deg}_{y} c_{k} \leq 2 k, 0 \leq k \leq m \text {. }
$$

Alors, si on fait le changement de variable dépendante $y_{1}=1 / y$ on obtient une équation polynomiale

$$
G\left(x, y_{1}, y_{1}^{\prime}\right)=y_{1}^{2 m} F\left(x, \frac{1}{y_{1}},-\frac{y_{1}^{\prime}}{y_{1}^{2}}\right)=0
$$

avec

$$
G\left(x, y_{1}, z_{1}\right)=\sum_{k=0}^{m} d_{k}\left(x, y_{1}\right) z_{1}^{m-k}
$$

où

$$
d_{k}\left(x, y_{1}\right)=(-1)^{m-l} c_{k}\left(x, 1 / y_{1}\right) y_{1}^{2 k} .
$$

Par hypothèse (V.10) admet une solution transcendante uniforme $y_{1}=u(x)$ n'ayant qu'un nombre fini de zéros. On applique le théorème à (V.10) avec $P\left(x, y_{1}\right)=y_{1}$. On en déduit que $y_{1}=0$ est une solution de (V.10). Donc $d_{m}(x, 0)=0$. Alors, $\operatorname{deg}_{y} c_{m}<2 m$. Le reste du corollaire suit du théorème appliqué à V.10 en tenant compte du fait que la courbe $G\left(a, y_{1}, z_{1}\right)=0$ correspond avec la courbe $F(a, y, z)=0$ via de la transformation birationnelle

$$
y=1 / y_{1}, z=-z_{1} / y_{1}^{2}
$$

Théorème V.4.10. Supposons que l'équation (V.3) admette une solution transcendante uniforme $y=u(x)$ n'ayant qu'un nombre fini de zéros et de pôles. Alors (V.3) est une équation linéaire homogène à coefficients rationnels. En particulier, cette solution est de la forme

$$
u(x)=R(x) e^{K(x)}
$$

où $R$ est une fonction rationnelle et $K$ est un polynôme non-constant. 
Pour démontrer le théorème on a besoin des deux lemmes suivants.

Lemme V.4.11. Considérons deux équations différentielles polynomiales

$$
F\left(x, y, y^{\prime}\right)=0, G\left(x, y, y^{\prime}\right)=0 .
$$

Supposons qu'il existe un ouvert non-vide $U \subset \mathbb{C}$ et une infinité de fonctions méromorphes sur $U$ :

$$
y=y_{k}(x), k=1,2,3, \ldots
$$

qui sont des solutions des deux équations simultanément. Alors, il existe $c \in \mathbb{C}-\{0\}$ tel que

$$
G(x, y, z)=c F(x, y, z)
$$

Démonstration. Désignons $E_{k}$ l'ensemble des pôles de $y_{k}(x)$ et posons

$$
E_{i j}:=\left\{x \in U: y_{i}(x)=y_{j}(x)\right\}, i \neq j .
$$

Alors,

$$
T:=\left(\bigcup_{k} E_{k}\right) \cup\left(\bigcup_{i \neq j} E_{i j}\right)
$$

est un sous-ensemble dénombrable de $U$. Soient $S_{F}$ et $S_{G}$ les surfaces algébriques irréductibles de $\mathbb{C}^{3}$ définies par les équations $F=0$ et $G=0$ respectivement. Si $x \in U-T$, alors

$$
\left(x, y_{k}(x), y_{k}^{\prime}(x)\right) \in S_{F} \cap S_{G}
$$

pour tout $k=1,2,3, \ldots$. Cela nous dit que $S_{F} \cap S_{G}$ n'est pas vide et n'est pas une courbe algébrique. Alors $S_{F}=S_{G}$, d'où suit le lemme.

Lemme V.4.12. Soit $y=v(x)$ une fonction entière non-constante et soit $y=p(x)$ un polynôme. Supposons qu'il existe une fonction rationnelle $R(x, y)$ telle que

$$
e^{v(x)}=R\left(x, e^{p(x)}\right) .
$$

Alors $R(x, y)=c y^{n}$ pour $c \in \mathbb{C}-\{0\}$ et $n \in \mathbb{Z}-\{0\}$.

Démonstration. Observons d'abord que $p(x)$ n'est pas constant. Écrivons $R(x, y)$ comme fraction irréductible

$$
R(x, y)=\frac{A(x, y)}{B(x, y)}, A, B \in \mathbb{C}[x, y] .
$$

Comme le système d'équations

$$
A(x, y)=0, B(x, y)=0
$$


n'a qu'un nombre fini de solutions et que exp $v(x)$ n'a ni zéro ni pôle, on conclut que chacune des fonctions

$$
A(x, \exp p(x)), B(x, \exp p(x))
$$

n'a qu'un nombre fini de zéros. On applique le théorème V.4.5 à l'équation linéaire

$$
y^{\prime}-p(x) y=0
$$

et à la solution $y=\exp p(x)$. Alors, si $P(x, y)$ est un facteur irréductible de $A(x, y)$ ou de $B(x, y)$ qui dépend effectivement de $y$, on en déduit que $P(x, y)=0$ est une solution algébrique de (V.11). Mais la seule solution algébrique de (V.11) est $y=0$, donc $P=a y, a \in \mathbb{C}, a \neq 0$. Alors

$$
R(x, y)=S(x) y^{n},
$$

pour une fonction rationnelle $S(x)$ et $n \in \mathbb{Z}$. Mais

$$
e^{\mathrm{v}(x)}=S(x) e^{\mathrm{n} p(x)}
$$

implique que $S$ n'a ni zéros ni pôles. Donc $S(x)$ est une fonction constante non-nulle.

Preuve du théorème V.4.10. La preuve se déroule de manière tout-à-fait analogue à celle du théorème V.4.5 avec $P(x, y)=y$ mais, dans ce cas, on considère aussi une courbe algébrique fermée irréductible $C^{\prime} \subset X$ telle que $g^{\prime}\left(C^{\prime}\right)=\mathbb{P}_{1} \times\{\infty\}$. Comme pour $C$, on prouve que $C^{\prime}$ est invariante par $\mathcal{F}$. Alors, au lieu d'une solution algébrique de (V.9) nous en avons deux (dont l'une peut être $y=\infty$; dans ce cas (V.9) est linéaire). Puisque trois solutions différentes de (V.9) déterminent rationnellement toutes les autres, ces deux solutions doivent être de degré 1 , étant donné qu'il existe une solution transcendante (théorème III.5.7). Donc, ces deux solutions algébriques sont rationnelles. Dans tous les cas, on obtient que (V.3) se ramène rationnellement à une équation homogène à coefficients rationnels

$$
s^{\prime}-q(x) s=0
$$

au moyen d'un changement de la variable dépendante $y=A(x, s)$, comme dans la définition V.4.4. Alors, il existe une fonction $s=w(x)$ méromorphe sur $\mathbb{C}$, solution de (V.12), telle que

$$
u(x)=A(x, w(x)) .
$$

D'autre part,

$$
u(x)=R(x) e^{K(x)} \text { et } w(x)=T(x) e^{L(x)}
$$


où $R, T$ sont des fonctions rationnelles non-nulles, $K$ est une fonction entière et $L$ un polynôme; de plus, $K$ et $L$ sont non-constantes. Alors

$$
e^{K(x)}=\frac{A\left(x, T(x) e^{L(x)}\right)}{R(x)} .
$$

D'après le lemme V.4.12 appliqué à la fonction rationnelle $A(x, T(x) z) / R(x)$ on a

$$
\frac{A(x, T(x) z)}{R(x)}=c z^{n}, c \in \mathbb{C}-\{0\}, n \in \mathbb{Z} .
$$

En posant $s:=T(x) z$, il en résulte

$$
A(x, s)=B(x) s^{n}
$$

où $B$ est une fonction rationnelle et $n \in \mathbb{Z}$. De plus, comme $A$ dépend effectivement de $s$, on a que $B$ et $n$ sont non-nuls. Mais si $s=s(x)$ est une solution de (V.12) alors $y=B(x) s(x)^{n}$ est une solution de

$$
y^{\prime}=\frac{B^{\prime}(x)+n q(x) B(x)}{B(x)} y .
$$

En même temps, $y=B(x) S(x)^{m}=A(x, S(x))$ est une solution de (V.3) d'après la définition V.4.4. La démonstration se termine par application du lemme V.4.11 aux équations (V.3) et (V.13). 


\section{Annexe A}

\section{Feuilletages analytiques et équations de Pfaff sur les surfaces complexes}

\section{A.1 Feuilletages réguliers}

Soit $X$ une surface analytique complexe régulière. Pour $p \in X$, on note $T_{p} X$ l'espace tangent de $X$ en $p$.

Définition A.1.1. Un champ analytique $L$ de droites tangentes à $X$ est une correspondance qui à chaque point $p \in X \overline{\text { associe un sous-espace vecto- }}$ riel $L_{p} \subset T_{p} X$ de dimension 1 (droite), telle que pour tout $q \in X$ il existe une 1-forme $\omega$ holomorphe et jamais nulle sur un voisinage ouvert $U$ de $q$ telle que $L_{p}=\operatorname{ker} \omega(p)$ pour tout $p \in U$. Dans cette situation on dit que $\omega=0$ est une équation locale de $L$.

Le problème qui se pose quand on a un tel champ $L$ est d'étudier la famille de courbes analytiques de $X$ tangentes à $L$.

Définition A.1.2. Une courbe tangente à L est un couple $(\Sigma, \varphi)$ où $\Sigma$ est une surface de Riemann $\overline{\text { et } \varphi: \Sigma \rightarrow X}$ est une application analytique et injective telle que $d \varphi(u)\left(T_{p} \Sigma\right)=L_{\varphi(u)}$ pour tout $u \in \Sigma$ (c'est-à-dire que $\varphi$ est une immersion).

Lemme A.1.3. Soit $\left(\Sigma_{1}, \varphi_{1}\right)$ une courbe tangente à $L$ et soit $\varphi_{2}: \Sigma_{2} \rightarrow$ $X$ une immersion injective de la surface de Riemann $\Sigma_{2}$. Supposons que $\varphi_{2}\left(\Sigma_{2}\right) \subset \varphi_{1}\left(\Sigma_{1}\right)$. Alors $V:=\varphi_{1}^{-1}\left(\varphi_{2}\left(\Sigma_{2}\right)\right)$ est un ouvert de $\Sigma_{1}$ et l'application $\Theta: V \rightarrow \Sigma_{2}$ définie par restriction de $\varphi_{2}^{-1} \circ \varphi_{1} \dot{a} V$ est holomorphe et bijective. En particulier $\left(\Sigma_{2}, \varphi_{2}\right)$ est une courbe tangente à $L$. 
Démonstration. Que $\Theta$ est bien définie et bijective résulte du fait que $\varphi_{1}$ et $\varphi_{2}$ sont injectives. Comme $\varphi_{1}$ est une immersion, elle admet un inverse local à gauche, holomorphe au voisinage de chaque point de $\varphi_{1}\left(\Sigma_{1}\right)$. Cela implique que l'application $\varphi_{1}^{-1} \circ \varphi_{2}: \Sigma_{2} \rightarrow \Sigma_{1}$ est holomorphe; comme elle est aussi injective, son image est un ensemble ouvert. Finalement, $\Theta$ est holomorphe parce que $\varphi_{2}$, étant une immersion, admet un inverse local à gauche qui est holomorphe au voisinage de chaque point de $\varphi_{2}\left(\Sigma_{2}\right)$.

Observation A.1.4. Dans le cas où $\varphi_{1}\left(\Sigma_{1}\right)=\varphi_{2}\left(\Sigma_{2}\right)$, le lemme précédent nous dit qu'une courbe tangente à $L$ est essentiellement déterminée par son image dans $X$.

Définition A.1.5. Un sous-ensemble $F$ de $X$ est une feuille tangente $\grave{a}$ $L$ s'il est l'image d'une courbe tangente à $L$ et il n'est strictement contenu dans l'image d'aucune courbe tangente à L. L'ensemble des feuilles tangentes à $L$ est un feuilletage régulier de $X$ (défini par $L$ ). Si $p \in F$, la droite $L_{p}$ est la droite tangente ̀̀ $F$ en $p$. Le champ $L$ est le champ tangent aux feuilles $d u \overline{\text { feuilletage. Une courbe tangente au feuilletage ou simple- }}$ ment une feuille est une courbe tangente $\grave{a} L$.

Exemple A.1.6. Soient $S$ une surface de Riemann et $g: X \rightarrow S$ une application analytique telle que $d g(p)$ est surjectif pour tout $p \in X$. Alors, l'application qui à $p \in X$ associe $L_{p}:=\operatorname{ker} d g(p)$, définit un champ analytique de droites tangentes à $X$ dont les feuilles sont les composantes connexes des fibres de $g$.

Proposition A.1.7. Pour chaque $p \in X$ il existe un voisinage $U$ de $p$ et un difféomorphisme analytique $\alpha: \Delta \times \Delta \rightarrow U$, où $\Delta \subset \mathbb{C}$ est un disque ouvert, tel que

a) pour chaque $v \in \Delta$, l'application $\alpha_{v}: \Delta \rightarrow X$ définie par $\alpha_{v}(u)=$ $\alpha(u, v), u \in \Delta$, est une courbe tangente à $L$;

b) si $(\Sigma, \varphi)$ est une courbe tangente à $L$ et $\varphi(\Sigma) \subset U$, alors il existe $v \in \Delta$ tel que $\varphi(\Sigma) \subset \alpha(\Delta \times\{v\})$ et $\varphi(\Sigma)$ est ouvert dans $\alpha(\Delta \times\{v\})$.

Démonstration. Soit $\omega=0$ une équation locale de $L$ au voisinage de $p$. Si $(x, y)$ est un système de coordonnées locales de $X$ centré en $p$, on a

$$
\omega=a(x, y) d x+b(x, y) d y
$$

où $a, b$ sont des fonctions holomorphes au voisinage de $(0,0) \in \mathbb{C}^{2}$ et

$$
|a(x, y)|+|b(x, y)|>0 .
$$

Supposons, par exemple, que $b(0,0) \neq 0$. Alors, toute solution analytique $y=y(x)$ au voisinage de 0 de l'équation

$$
\frac{d y}{d x}=-\frac{a(x, y)}{b(x, y)}
$$


détermine une courbe tangente à $L$. Par conséquent, l'assertion a) suit du théorème d'existence, unicité et dépendance analytique des conditions initiales des solutions d'une équation différentielle analytique ordinaire (voir [18] ou [20]).

Par un argument de connexité, pour prouver la première affirmation de b), il suffit de considérer le cas où $\Sigma=D \subset \mathbb{C}$ est un disque de centre 0 . Soient $(x(t), y(t))$ les coordonnées de $\varphi(t), t \in D$, où $x=x(t), y=y(t)$ sont des fonctions analytiques sur $D$ et $\left|x^{\prime}(t)\right|+\left|y^{\prime}(t)\right|>0$. Alors

$$
a(x(t), y(t)) x^{\prime}(t)+b(x(t), y(t)) y^{\prime}(t)=0, t \in D .
$$

Comme on peut supposer $b(x, y) \neq 0$ en tout point de $U$, on a que $x^{\prime}(t) \neq$ 0 pour tout $t \in D$. Pour chaque $t_{0} \in D$, la fonction analytique $x=x(t)$ admet un inverse local analytique $t=t(x)$ au voisinage de $x_{0}=x\left(t_{0}\right)$. On voit tout de suite que $y=y(t(x))$ est une solution de (A.1). D'après le théorème d'unicité cité plus haut, il existe un voisinage $U_{t_{0}} \subset D$ de $t_{0}$ et $v_{0} \in \Delta$ tels que le point de $U$ de coordonnées $(x(t), y(t))$ appartient à $\alpha\left(\Delta \times\left\{v_{0}\right\}\right)$ pour tout $t \in U_{t_{0}}$.

La première affirmation de $\mathrm{b}$ ) en résulte aussitôt.

D'après A.1.3, l'ensemble $\alpha_{v}^{-1}(\varphi(\Sigma))=\alpha^{-1}(\varphi(\Sigma))$ est un ouvert de $\Delta \times$ $\{v\}$. Comme $\alpha$ est un homéomorphisme, $\varphi(\Sigma)$ est un ouvert de $\alpha(\Delta \times\{v\})$.

Corollaire A.1.8. Soient $\left(\Sigma_{1}, \varphi_{1}\right),\left(\Sigma_{2}, \varphi_{2}\right)$ deux courbes tangentes à L. On a les assertions suivantes:

a) l'ensemble $V:=\varphi_{1}^{-1}\left(\varphi_{2}\left(\Sigma_{2}\right)\right)$ est un ouvert de $\Sigma_{1}$;

b) l'application $\left(\varphi_{2}^{-1}\right) \circ\left(\left.\varphi_{1}\right|_{V}\right): V \rightarrow \Sigma_{2}$ est analytique;

c) l'intersection $\varphi_{1}(\Sigma) \cap \varphi_{2}\left(\Sigma_{2}\right)$ est une réunion disjointe d'images de courbes tangentes à $L$.

Démonstration. a) Soit $\xi \in V$; notons $p:=\varphi_{1}(\xi)$ et $\eta:=\varphi_{2}^{-1}(p)$. Prenons un disque ouvert $\Delta \subset \mathbb{C}$, un voisinage $U$ de $p$ dans $X$ et un difféomorphisme analytique $\alpha: \Delta \times \Delta \rightarrow U$ satisfaisant a) et b) de la proposition A.1.7. Soit $v \in \Delta$ un point tel que $p \in \alpha(\Delta \times\{v\})$.

Soient $V_{1}, V_{2}$ des voisinages ouverts de $\xi, \eta$ dans $\Sigma_{1}, \Sigma_{2}$ respectivement, 
tels que $\varphi_{1}\left(V_{1}\right) \subset U$ et $\varphi_{2}\left(V_{2}\right) \subset U$.

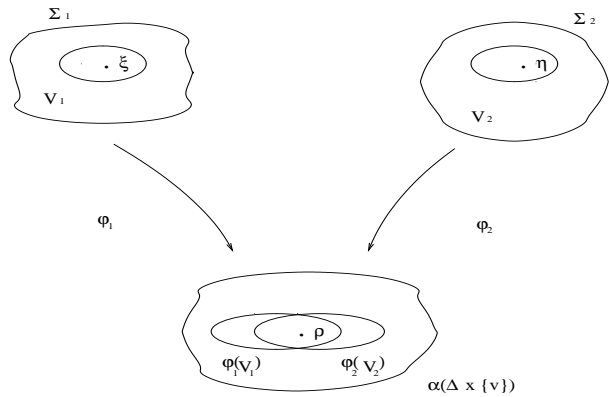

Figura A.1

Comme $\left(V_{1},\left.\varphi_{1}\right|_{V_{1}}\right)$ et $\left(V_{2},\left.\varphi_{2}\right|_{V_{2}}\right)$ sont des courbes tangentes à $L$, on a

$$
\varphi_{1}\left(V_{1}\right) \cup \varphi_{2}\left(V_{2}\right) \subset \alpha(\Delta \times\{v\}) .
$$

Par ailleurs, puisque $\varphi_{2}\left(V_{2}\right)$ est ouvert dans $\alpha(\Delta \times\{v\})$, l'ensemble $\varphi_{1}\left(V_{1}\right) \cap \varphi_{2}\left(V_{2}\right)$ est ouvert dans $\varphi_{1}\left(V_{1}\right)$. Alors,

$$
W:=\left(\left.\varphi_{1}\right|_{V_{1}}\right)^{-1}\left(\varphi_{1}\left(V_{1}\right) \cap \varphi_{2}\left(V_{2}\right)\right)
$$

est ouvert dans $V_{1}$; donc il l'est aussi dans $\Sigma_{1}$. De plus, $\xi \in W \subset V$, ce qui prouve a).

b) et c). Soit $V=\left\{W_{i}\right\}_{i \in I}$ la décomposition de $V$ en composantes connexes. Chaque paire $\left(W_{i},\left.\varphi_{1}\right|_{W_{i}}\right)$, avec $i \in I$, est une courbe tangente à $L$. Alors, il suit de A.1.3 que $\left.\varphi_{2}^{-1} \circ \varphi_{1}\right|_{W_{i}}$ est holomorphe pour tout $i \in I$, car l'inverse d'une application holomorphe bijective est holomorphe. Ceci implique b). Finalement, on a évidemment

$$
\begin{aligned}
\varphi_{1}\left(V_{1}\right) \cap \varphi_{2}\left(V_{2}\right) & =\varphi_{1}(V) \\
& =\bigcup_{i \in I} \varphi_{1}\left(W_{i}\right),
\end{aligned}
$$

ce qui entraîne c).

Théorème A.1.9. On a les assertions suivantes:

a) Les feuilles d'un feuilletage régulier de $X$ sont des ensembles connexes par arcs, deux à deux disjoints et dont la réunion est $X$. L'image d'une courbe tangente aux feuilles est contenue dans une feuille.

b) La relation d'équivalence définie par la décomposition de $X$ en feuilles est ouverte. 
Démonstration. Il résulte de A.1.7 et A.1.8c que la famille des images de courbes tangentes à $L$ satisfait les conditions pour être une base d'ouverts d'une topologie sur $X$ et que celle-ci est plus fine que la topologie donnée sur $X$; on note $X^{\prime}$ l'ensemble $X$ muni de cette topologie.

a) Il suffit de démontrer que les feuilles du feuilletage sont les composantes connexes de $X^{\prime}$ (que chaque feuille soit connexe par arcs résulte du fait que les feuilles sont des images continues de surfaces de Riemann, par définition).

Observons d'abord que si $(\Sigma, \varphi)$ est une courbe tangente à $L$, alors $\varphi$ : $\Sigma \rightarrow X^{\prime}$ est continue d'après A.1.8a. Donc $\varphi(\Sigma)$ est connexe dans $X^{\prime}$; en particulier, toute feuille est contenue dans une composante connexe de $X^{\prime}$. Pour compléter la preuve de a) il suffit de prouver que toute composante connexe de $X^{\prime}$ est l'image d'une courbe tangente à $L$.

Soit $F$ une composante connexe de $X^{\prime}$ munie de la topologie induite par celle de $X^{\prime}$. On va munir $F$ d'une structure de surface de Riemann telle que $(F, \imath)$ est une courbe tangente à $L$, où $\imath: F \rightarrow X$ désigne l'inclusion. Pour cela on prend comme cartes locales de cette structure, les couples $(\Delta, \varphi)$, où $\Delta \subset \mathbb{C}$ est un disque ouvert avec $\varphi(\Delta) \subset F$ et $(\Delta, \varphi)$ est une courbe tangente à $L$. D'après A.1.7a et ce que l'on vient de voir, ces cartes locales sont des homéomorphismes sur des ouverts de $F$ qui recouvrent $F$. Par A.1.8b, les fonctions de transition sont analytiques, d'où l'assertion a).

b) On note $\sim$ la relation d'équivalence et pour tout $S \subset X$ on désigne par $S^{\sim}$ le saturé par $\sim$. Il résulte de la proposition A.1.7 que $X$ peut être recouvert par une famille $\Re$ d'ouverts telle que si $U \in \Re$, alors pour tout $p, q \in U$ avec $p \sim q$ et que pour tout voisinage ouvert $V_{p} \subset U$ de $p$, on a que $q$ est intérieur à $V_{p}^{\sim}$.

Considérons, maintenant, un ouvert quelconque $U \subset X$ et soit $p \in U$, $q \in X$ avec $p \sim q$. Par a) il existe un chemin continu $\gamma:[0,1] \rightarrow X$ tel que $\gamma(0)=p, \gamma(1)=q$ et $\gamma(t) \sim p$ pour tout $t \in[0,1]$. Il existe une partition $0=t_{0}<t_{1}<\cdots<t_{n}=1$ telle que

$$
\gamma\left(\left[t_{j-1}, t_{j}\right]\right) \subset U_{j} \in \Re, 1 \leq j \leq n .
$$

On voit par récurrence que $\gamma\left(t_{j}\right)$ est intérieur à $U^{\sim}$ pour $j=1, \ldots, n$. Donc, $q$ est aussi un point intérieur à $U^{\sim}$.

Exemple A.1.10. Soit $X \subset \mathbb{C}^{2}$ un ouvert connexe et soit $\omega$ une 1-forme holomorphe jamais nulle sur $X$. Alors $p \mapsto \operatorname{ker} \omega(p)$ définit un champ analytique de droites tangentes à $X$. Supposons que $d \omega=0$. Par intégration de $\omega$ on obtient une fonction multiforme $f$ sur $U$. Les composantes connexes des courbes de niveau de chaque détermination uniforme locale de $f$ sont des images de courbes tangentes à $L$. On peut interpréter les feuilles du feuilletage défini par $L$ comme étant les courbes de niveau de $f$. 
Définition A.1.11. Soit $F$ une feuille du feuilletage défini par L. Soit $(\Sigma, \varphi)$ une courbe tangente à $L$ telle que $\varphi(\Sigma)=F$. On définit la topologie fine de $F$ comme étant celle dont les ouverts sont les ensembles $\varphi \overline{(U) \text { pour }}$ $\bar{U}$ ouvert dans $\Sigma$ (elle est bien définie : voir observation A.1.4). Si la topologie fine de $F$ coïncide avec celle induite par $X$ on dit que $F$ est une feuille propre.

Lemme A.1.12. Soit $W \subset X$ un ouvert. Alors, les feuilles du feuilletage de $W$ défini par $\left.L\right|_{W}$ sont les composantes connexes, pour la topologie fine, de l'intersection avec $W$ des feuilles du feuilletage de $X$ défini par $L$.

Démonstration. Soit $F$ une feuille de $X$. Il est clair, par définition, que chaque composante connexe $F_{i}$ de $F \cap W$, pour la topologie fine de $F$, est l'image d'une courbe tangente à $\left.L\right|_{W}$.

Si $C$ est l'image d'une courbe tangente à $L$ et $F_{i} \subset C \subset W$, alors $C$ est ouvert et connexe dans $F \cap W$ pour la topologie fine de $F$ par A.1.7a et A.1.8a. Alors $F_{i}=C$, ce qui complète la preuve.

Définition A.1.13. Dans les conditions de A.1.12, on dit que le feuilletage de $W$ est la restriction de celui de $X$.

D'après les définitions ci-dessus on obtient tout de suite le lemme suivant.

Lemme A.1.14. Soit $\left\{U_{i}\right\}_{i \in I}$ un recouvrement de $X$ par des ouverts connexes. Supposons donné un feuilletage régulier $\mathcal{F}_{i}$ de chaque $U_{i}$ de telle façon que

$$
\left.\mathcal{F}_{i}\right|_{U_{i} \cap U_{j}}=\left.\mathcal{F}_{j}\right|_{U_{i} \cap U_{j}} \text {, pour tout } i, j \in I .
$$

Alors, il existe un feuilletage régulier $\mathcal{F}$ de $X$ tel que $\left.\mathcal{F}\right|_{U_{i}}=\mathcal{F}_{i}$, pour tout $i \in I$.

Observation A.1.15. $X$ est une variété réelle de dimension 4. Tout feuilletage régulier de $X$ définit un feuilletage réel de $X$ de dimension 2(voir [7]).

Le lemme suivant sera utilisé plus tard.

Lemme A.1.16. Supposons donné un feuilletage régulier de $X$. Soit $Y$ un espace topologique localement connexe et soit $f: Y \rightarrow X$ une application continue telle que $f(Y)$ est contenu dans une feuille $F$. Alors $f: Y \rightarrow F$ est continue pour la topologie fine de $F$.

Démonstration. On peut supposer que $Y$ est connexe et que $f(Y) \subset U$ où $U$ est un ouvert comme dans la proposition A.1.7

Par définition de la topologie fine, $F \cap \alpha(\{0\} \times \Delta)$ est un sous-ensemble discret de $F$ pour celle-ci. En particulier cet ensemble est dénombrable. Alors

$$
F \cap U=\alpha(\Delta \times S)
$$


où $S \subset \Delta$ est un ensemble dénombrable. Il en résulte que chaque composante connexe de $F \cap U$, pour la topologie induite par celle de $X$, est de la forme $\alpha(\Delta \times\{v\}), v \in \Delta$. Alors, $f(Y) \subset \alpha\left(\Delta \times\left\{v_{0}\right\}\right)$ pour un $v_{0} \in \Delta$. Comme la topologie fine de $F$ et la topologie de $M$ induisent la même topologie sur $\alpha\left(\Delta \times\left\{v_{0}\right\}\right)$ le lemme en résulte aussitôt.

\section{A.2 Feuilletages à singularités isolées}

Comme au $\S 1$ on désigne par $X$ une surface analytique régulière.

Définition A.2.1. Un feuilletage à singularités isolées de $X$ est un feuilletage régulier $\mathcal{F}$ de $X-\bar{S}$, où $S$ est un sous-ensemble discret et fermé de $X$.

Définition A.2.2. Un point $p \in S$ est un point singulier de $\mathcal{F}$ s'il n'existe

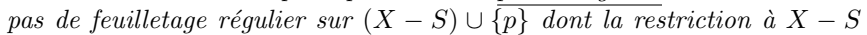
soit $\mathcal{F}$.

D'après A.1.14 on peut supposer que chaque point de $S$ est un point singulier de $\mathcal{F}$. Si $S$ est vide, on a donc un feuilletage régulier de $X$.

Théorème A.2.3. Soit $p$ un point singulier de $\mathcal{F}$. Alors, il existe un voisinage ouvert connexe $V$ de $p$ et une 1-forme holomorphe $\omega$ sur $V$ tels que:

a) si $q \in V$ et $q \neq p$, alors $q$ est non-singulier pour $\mathcal{F}$;

b) $\omega(p)=0$;

c) $\omega(q) \neq 0$ si $q \in V, q \neq p$ et le champ de droites $L_{q}:=\operatorname{ker} \omega(q)$, avec $q \in V-\{p\}$, est celui des droites tangentes aux feuilles de $\left.\mathcal{F}\right|_{V-\{p\}}$.

Démonstration. Soit $U \ni p$ un ouvert connexe, domaine d'un système de coordonnées locales $(x, y)$ de $X$ centré en $p$ et tel que $\left.\mathcal{F}\right|_{U-\{p\}}$ est un feuilletage régulier défini par un champ de droites $L$. Alors

$$
U-\{p\}=\bigcup_{i \in I} U_{i}
$$

où les $U_{i}$ sont des ouverts connexes, et dans chaque $U_{i}$ on a une 1-forme holomorphe jamais nulle $\omega_{i}$ telle que

$$
L_{q}:=\operatorname{ker} \omega_{i}(q), q \in U_{i}
$$

Ceci entraîne que

$$
\operatorname{ker} \omega_{i}(q)=\operatorname{ker} \omega_{j}(q) \text { si } q \in U_{i} \cap U_{j}(i, j \in I) .
$$

Donc $\omega_{i} \wedge \omega_{j}=0$ sur $U_{i} \cap U_{j}$. 
Écrivons

$$
\omega_{i}=a_{i}(x, y) d x+b_{i}(x, y) d y
$$

alors $a_{i} b_{j}-a_{j} b_{i}=0$ sur $U_{i} \cap U_{j}$. Supposons, par exemple, que $b_{j} \neq 0$. Par connexité de $U-\{p\}$ on a que $b_{i} \neq 0$ pour tout $i \in I$. Alors $h_{i}:=a_{i} / b_{i}$ est une fonction méromorphe sur $U_{i}$ et

$$
\left.h_{i}\right|_{U_{i} \cap U_{j}}=\left.h_{j}\right|_{U_{i} \cap U_{j}}, i, j \in I .
$$

Donc, il existe $h$ méromorphe sur $U-\{p\}$ telle que $\left.h\right|_{U_{i}}=h_{i}$ pour tout $i \in I$. On sait que $h$ se prolonge à une fonction méromorphe sur $U$, qu'on notera encore $h([15$, Vol. II, thm. O6]).

Il existe un voisinage ouvert et connexe $V$ de $p$ tel que $\left.h\right|_{V}=f / g$ où $f, g$ sont des fonctions holomorphes sur $V$ qui ne s'annulent pas simultanément en dehors de $p$. Considérons la 1-forme

$$
\omega=f(x, y) d x+g(x, y) d y
$$

qui est holomorphe sur $V$ et non-nulle sur $V-\{p\}$. Par construction on a $\omega \wedge \omega_{i}=0$ sur $U_{i} \cap V$, ce qui implique

$$
\operatorname{ker} \omega(q)=\operatorname{ker} \omega_{i}(q)=L_{q} \text { pour } q \in U_{i} \cap V .
$$

On en déduit

$$
\operatorname{ker} \omega(q)=L_{q} \text { pour } q \in V-\{p\} .
$$

Finalement, $\omega(p)=0$ parce que $p$ est un point singulier de $\mathcal{F}$.

Exemple A.2.4. Supposons $X$ connexe. Soit $B$ une surface de Riemann et soit $h: X \rightarrow B$ holomorphe propre et non-constante. On note $X_{b}:=h^{-1}(b)$ la fibre de $h$ au-dessus du point $b \in B$.

Si $p$ est un point régulier de la courbe $X_{b}$ on pose

$$
L_{p}:=T_{p}\left(X_{b}\right) .
$$

Soit $z$ une coordonnée locale de $B$ centrée en $b$ et soit $\varphi:=z \circ h$. Comme le germe en $p$ de l'ensemble $\varphi=0$ est régulier, $\varphi=\theta^{k}$ où $\theta$ est holomorphe au voisinage de $p$, s'annule en $p$ et $d \theta(p) \neq 0$. Alors, les ensembles $\{\theta=c t e$. $\}$ sont réguliers au voisinage de $p$. Donc, $q$ est un point régulier de $X_{h(q)}$ pour tout $q$ au voisinage de $p$ et $L_{q}:=\operatorname{ker} d \theta(q)$.

Le fait que $h$ est propre montre tout de suite que l'ensemble $S$ des points singuliers des courbes $X_{b}, b \in B$, est fermé et discret dans $X$, et que $L$ est un champ analytique de droites tangentes à $X-S$.

Enfin, nous avons un feuilletage à singularités isolées de $X$ dont les points singuliers sont les singularités des fibres de $h$ et dont les feuilles sont les composantes connexes des ensembles des points réguliers de celles-ci : c'est le feuilletage défini par $h$. 
Observation A.2.5. On définit, comme dans A.1.13, la notions de restriction à un ouvert de $X$ d'un feuilletage à singularités isolées et on a le lemme analogue à A.1.14.

Le lemme suivant suit directement les définitions ci-dessus.

Lemme A.2.6. Soient $\mathcal{F}$ et $\mathcal{F}^{\prime}$ deux feuilletages à singularités isolées de $X$ et supposons qu'il existe un ouvert connexe et non-vide $W \subset X$ tel que $\left.\mathcal{F}\right|_{W}=\left.\mathcal{F}^{\prime}\right|_{W}$. Alors, si $X$ est connexe, $\mathcal{F}=\mathcal{F}^{\prime}$.

\section{A.3 Les équations de Pfaff}

Soit $\omega \neq 0$ une 1-forme méromorphe sur la surface analytique complexe, régulière et connexe $X$.

Proposition A.3.1. Il existe un sous-ensemble $S$ discret et fermé de $X$ et un champ analytique $L$ de droites tangentes $\grave{a} X-S$ tels que si $\omega$ est holomorphe au voisinage d'un point $p \in X$ et $\omega(p) \neq 0$, alors $p \notin S$ et $L_{p}=\operatorname{ker} \omega(p)$.

Démonstration. Notons $U \subset X$ l'ouvert de Zariski constitué des points où $\omega$ est holomorphe et non-nulle; on sait que $U$ est connexe et dense dans $X$; on pose

$$
L_{p}:=\operatorname{ker} \omega(p), p \in U
$$

On en déduit qu'il existe un ouvert maximal $W$, avec $U \subset W \subset X$, sur lequel $L$ se prolonge. Il suffit de prouver que $S=X-W$ est discret.

Soient $p \in S$ et $(x, y)$ un système de coordonnées de $X$ centré en $p$. Au voisinage de $p$ on peut écrire $\omega$ sous la forme

$$
\omega=a(x, y) d x+b(x, y) d y
$$

où $a, b$ sont des fonctions méromorphes. Par ailleurs, l'anneau local de $X$ en $p$ est factoriel; on note $g$ le plus grand diviseur commun des germes de $a$ et $b$ en $p$. On déduit que $\omega=g \omega^{\prime}$ où $\omega^{\prime}$ est holomorphe au voisinage de $p$ et ne s'annule pas en dehors de $p$.

On définit $L_{q}=\operatorname{ker} \omega^{\prime}(q)$, pour $q \neq p$ au voisinage de $p$ et on obtient un prolongement de $L$, car $\operatorname{ker} \omega^{\prime}(q)=\operatorname{ker} \omega(q)$ dans l'ouvert dense, dans ce voisinage de $p$, où $a, b, g$ sont holomorphes et où $g$ et $a$ ou $b$ ne s'annulent pas. Donc $p$ est isolé dans $S$, ce qui termine la preuve.

Définition A.3.2. Dans les conditions de A.3.1, on dit que le feuilletage à singularités isolées défini par $L$ est le feuilletage de $X$ défini par l'équation de Pfaff $\omega=0$. 
Exemple A.3.3. Soit $X=\mathbb{C}^{2}$ et $\omega=\left(y^{2}-1\right) d x-x[A(y+1)+B(y-1)] d y$ où $A, B \in \mathbb{C}$ sont tels que le sous-groupe additif de $\mathbb{C}$ engendré par $1, A, B$ soit dense dans $\mathbb{C}$ (voir $[10])$.

Soient $z_{1}:=\exp (2 \pi i A), z_{2}:=\exp (2 \pi i B)$. Observons que $z_{1}^{n} z_{2}^{m} \neq 1$ si $n^{2}+m^{2}>0$ pour $n, m \in \mathbb{Z}$ et que l'ensemble

$$
D:=\left\{z_{1}^{n} z_{2}^{m}: n, m \in \mathbb{Z}\right\}
$$

est dense dans $\mathbb{C}$, car il est l'image du sous-groupe additif engendré par $1, A, B$ par l'application $w=\exp (2 \pi i z)$.

Choisissons une détermination locale, au voisinage de 0 , de $A \log (y-1)$ et de $B \log (y+1)$. Leur somme admet un prolongement analytique le long de chaque courbe d'origine 0 contenue dans $U:=\mathbb{C}-\{1,-1\}$.

Alors, la surface de Riemann $\Sigma$ de la fonction

$$
A \log (y-1)+B \log (y+1)
$$

est un revêtement non-ramifié $\pi: \Sigma \rightarrow U$ et il existe $f: \Sigma \rightarrow \mathbb{C}$ holomorphe, telle que toute détermination locale de (A.2) est de la forme $f \circ \pi^{-1}$, avec $\pi^{-1}$ un inverse local de $\pi$; considérons l'application $\varphi: \Sigma \rightarrow X$ donnée par

$$
\varphi(\xi)=(\exp f(\xi), \pi(\xi)) .
$$

Alors $\varphi(\Sigma) \subset \mathbb{C} \times U$, qui est l'ouvert où $\omega$ est holomorphe et jamais nulle. Nous allons vérifier que $(\Sigma, \varphi)$ est une courbe tangente au champ $L$ de droites tangentes à $\mathbb{C} \times U$, défini par

$$
L(x, y)=\operatorname{ker} \omega(x, y),(x, y) \in \mathbb{C} \times U .
$$

1) $\varphi$ est injective. Soient $\xi, \xi^{\prime} \in \Sigma$ avec $\varphi(\xi)=\varphi\left(\xi^{\prime}\right)$. Alors $\pi(\xi)-\pi\left(\xi^{\prime}\right)=0$ et $\exp \left(f(\xi)-f\left(\xi^{\prime}\right)\right)=1$. Mais $\pi(\xi)=\pi\left(\xi^{\prime}\right)$ entraîne $f(\xi)-f\left(\xi^{\prime}\right)=2 \pi i(n A+$ $m B$ ) pour certains $n, m \in \mathbb{Z}$. Donc $z_{1}^{n} z_{2}^{m}=1$, d'où suit aussitôt $n=m=0$. On conclut que $\xi, \xi^{\prime}$ correspondent à la même détermination locale de la fonction (A.2) ; c'est-à-dire, $\xi=\xi^{\prime}$.

2) $\varphi$ est une immersion. Cela vient du fait que $\pi$ est un difféomorphisme local.

3) $d \varphi\left(T_{\xi}(\Sigma)\right) \subset L_{\varphi(\xi)}$ parce que $\varphi^{*}(\omega)=0$. En effet, si $\pi^{-1}: W \rightarrow \Sigma$ est un inverse local de $\pi$ définie sur l'ouvert $W \subset U$, on vérifie toute de suite que $\left(\pi^{-1}\right)^{*}\left(\varphi^{*}(\omega)\right)=0$.

Maintenant on va vérifier que $\varphi(\Sigma)$ est dense dans $X$; cela veut dire que le feuilletage de $X$ défini par l'équation de Pfaff $\omega=0$ possède une feuille dense.

Il suffit de voir que $\varphi(\Sigma)$ est dense dans $\mathbb{C} \times U$.

Prenons $y \in U$. L'ensemble

$$
E:=\{(\exp f(\xi), y): \xi \in \Sigma, \pi(\xi)=y\}
$$


est contenu dans $\varphi(\Sigma)$. D'autre part, il existe $a \in \mathbb{C}$ tel que

$$
\left\{\exp (a) z_{1}^{n} z_{2}^{m}: n, m \in \mathbb{Z}\right\}
$$

est la projection de $E$ sur le premier facteur. Cet ensemble est donc dense dans $\mathbb{C}$, d'où l'assertion.

Théorème A.3.4. Supposons que $X$ est une variété algébrique projective et soit $\mathcal{F}$ un feuilletage à singularités isolées de $X$. Alors, il existe une 1 forme méromorphe non-nulle $\omega$ sur $X$ telle que $\mathcal{F}$ est défini par l'équation de Pfaff $\omega=0$.

Démonstration. Soit $S$ un sous-ensemble fermé et discret de $X$ et soit $L$ un champ de droites tangentes à $X-S$ qui définit $\mathcal{F}$. Prenons deux fonctions méromorphes $f, g \in \mathbb{C}(X)$ qui soient algébriquement indépendantes ([2, chap. I, $\S 7$ et chap. III, cor. 5.5]); un calcul direct montre que $d f \wedge d g \neq 0$. Il existe un ouvert de Zariski non-vide $U \subset X$ tel que $\left.f\right|_{U}$ et $\left.g\right|_{U}$ sont holomorphes, $d f \wedge d g$ ne s'annule jamais sur $U$ et $U \subset X-S$. Alors $U=$ $\cup_{i \in I} U_{i}$, où les $U_{i}$ sont des ouverts connexes sur lesquels il existe une 1-forme holomorphe jamais nulle $\omega_{i}$ telle que

$$
L_{q}=\operatorname{ker} \omega_{i}(q), q \in U_{i}(i \in I) .
$$

On écrit

$$
\omega=a_{i} d f+b_{i} d g
$$

où $a_{i}, b_{i}$ sont holomorphes sur $U_{i}$ pour tout $i \in I$. Comme $\omega_{i} \wedge \omega_{j} \neq 0$ sur $U_{i} \cap U_{j}$, on a

$$
a_{i} b_{j}-a_{j} b_{i}=0 \text { sur } U_{i} \cap U_{j}, i, j \in I .
$$

Supposons, par exemple, $b_{j} \neq 0$. Alors, par la connexité de $U$, on obtient que $b_{i} \neq 0$ pour tout $i \in I$. Ceci permet de définir, pour chaque $i \in I$ une fonction méromorphe $h_{i}:=a_{i} / b_{i}$ sur $U_{i}$ de sorte que

$$
\left.h_{i}\right|_{U_{i} \cap U_{j}}=\left.h_{j}\right|_{U_{i} \cap U_{j}}, i, j \in I .
$$

Donc, il existe une fonction méromorphe $h$ sur $U$ telle que $\left.h\right|_{U_{i}}=h_{i}$ pour tout $i \in I$. Considérons la 1 -forme méromorphe sur $U$ définie par

$$
\omega:=h d f+d g
$$

alors $\left.b_{i} \omega\right|_{U_{i}}=\omega_{i}$ pour tout $i \in I$.

Soit $p \in U_{i}$ tel que $b_{i}(p) \neq 0$. Alors $\omega$ est holomorphe au voisinage de $p$ et

$$
\operatorname{ker} \omega(p)=\operatorname{ker} \omega_{i}(p)=L_{p} .
$$

Supposons que $h$ soit méromorphe sur $X$. Ce qui précède nous dit que l'équation de Pfaff $\omega=0$ définit le feuilletage $\mathcal{F}$ (voir A.2.3 et A.2.6). Il reste donc à démontrer que $h$ est méromorphe sur $X$. 
Soit $p \in X-U$. Par le théorème A.2.3 et les définitions, il existe un voisinage ouvert connexe $V$ de $p$ et une 1-forme $\theta$ holomorphe sur $V$ telle que ker $\theta(q)=L_{q}$ pour tout $q \in V-\{p\}$. Soit $W:=V \cap U$. Alors

$$
\omega \wedge \theta=0 \operatorname{sur} W \text {. }
$$

On en déduit que

$$
h(d f \wedge \theta)=-d g \wedge \theta \text { sur } W .
$$

Il suffit, alors, de prouver que $\left.d f\right|_{V} \wedge \theta \neq 0$.

Si $\left.d f\right|_{V} \wedge \theta=0$, on aurait

$$
\operatorname{ker} d f(q)=\operatorname{ker} \theta(q)=L_{q}, q \in W .
$$

Soit $j \in I$ tel que $U_{j} \cap V$ n'est pas vide. Il existe $r \in U_{j} \cap V$ tel que $b_{j}(r) \neq 0$. Comme on a vu plus haut, $\omega$ est holomorphe au voisinage de $r$ et $\operatorname{ker} \omega(r)=L_{r}$. Donc $\omega \wedge d f(r)=0$. Ceci implique $d g(r)=0$, ce qui est absurde.

Observation A.3.5. Si $u \neq 0$ est un champ de vecteurs méromorphe sur $X$ on prouve, comme dans A.3.1, qu'il existe un unique feuilletage de $X$ à singularités isolées tel que si $u$ est holomorphe au voisinage de $p \in X$ et $u(p) \neq 0$, alors $p$ est un point régulier du feuilletage et $u(p)$ est tangent à la feuille par $p$. On dit que ce feuilletage est défini par $u$.

\section{A.4 Séparatrices et intégrales premières}

On suppose donné un feuilletage analytique $\mathcal{F}$ à singularités isolées sur une surface analytique régulière $X$.

Définition A.4.1. Un germe irréductible $\Sigma$ d'un ensemble analytique de dimension 1 en un point $p \in X$ est une séparatrice locale de $\mathcal{F}$ en $p$ si $\Sigma-\{p\}$ est contenu dans une feuille de $\mathcal{F} . \overline{\text { On dit aussi que } \Sigma}$ est invariant $\operatorname{par} \mathcal{F}$.

Définition A.4.2. Un sous-ensemble analytique $Y$ de $X$, fermé, irréductible de dimension 1 est une séparatrice de $\mathcal{F}$ si pour tout $p \in Y$ chaque composante irréductible du germe de $Y$ en p est une séparatrice locale de $\mathcal{F}$ en $p$. On dit aussi que $Y$ est une courbe invariante par $\mathcal{F}$.

Lemme A.4.3. Soit $Y \subset X$ un sous-ensemble analytique fermé, irréductible de dimension 1. Supposons qu'il existe $p \in Y$ tel que l'une des composantes irréductibles du germe de $Y$ en p est une séparatrice locale de $\mathcal{F}$ en p. Alors, $Y$ est une séparatrice de $\mathcal{F}$.

Démonstration. Soit $L$ un champ analytique de droites tangentes à $X-S$ (où $S$ est un sous-ensemble fermé et discret de $X$ ) qui définit $\mathcal{F}$. Notons $Y_{0}$ l'ensemble des points singuliers de $Y$; posons $Y_{1}:=Y_{0}-S$ : c'est une 
surface de Riemann de sorte que l'inclusion $j: Y_{1} \rightarrow X$ est une immersion injective.

Par hypothèse, il existe un ouvert non-vide $U \subset Y_{1}$ tel que (voir A.1.3)

$$
\operatorname{dj}\left(T_{u}\left(Y_{1}\right)\right)=L_{j(u)}, \forall u \in U .
$$

Par analyticité, l'équation (A.3) vaut en fait pour tout $u \in Y_{1}$, et donc $Y_{1}$ est contenu dans une feuille de $\mathcal{F}$. Alors $\left(Y_{1}, j\right)$ est une courbe tangente à $L$. Le lemme en découle aussitôt.

Exemple A.4.4. Les séparatrices du feuilletage défini dans A.2.4 sont les composantes irréductibles des ensembles $h^{-1}(b)$ pour $b \in B$.

Définition A.4.5. Soit $X$ connexe et $f \in \mathbb{C}(X)$ non-constante; notons $U \subset X$ l'ouvert de Zariski constitué des points où $f$ est holomorphe. Alors $f$ est une intégrale première méromorphe de $\mathcal{F}$ si $\left.f\right|_{F \cap U}$ est constante pour toute feuille $F$ de $\mathcal{F}$.

Proposition A.4.6. Soient $\mathcal{F}$ un feuilletage analytique sur $X$ connexe et $f \in \mathbb{C}(X)$ non-constante. Supposons que $\mathcal{F}$ soit défini par une équation de Pfaff $\omega=0$. Alors, $f$ est une intégrale première méromorphe si et seulement $s i$

$$
d f \wedge \omega=0 .
$$

Démonstration. Supposons que $f$ soit une intégrale première de $\mathcal{F}$.

Soit $p \in X$ un point non-singulier de $\mathcal{F}$ tel que $\omega$ et $f$ sont holomorphes en $p$ et $\omega(p) \neq 0$. Une application directe de la proposition A.1.7 montre qu'il existe un voisinage ouvert $U$ de $p$ tel que

$$
d f(q)\left(L_{q}\right)=0, \forall q \in U,
$$

où $L$ est le champ analytique de droites défini par $\mathcal{F}$.

D'autre part, quitte à rétrécir $U$, nous pouvons supposer que $\omega(q)\left(L_{q}\right)=$ 0 pour tout $q \in U$, par la définition A.3.2; donc $\left.(d f \wedge \omega)\right|_{U}=0$, d'où suit l'assertion directe.

Réciproquement, supposons que $d f \wedge \omega=0$. Soit $p \in X$ un point nonsingulier de $\mathcal{F}$ en lequel $f$ est holomorphe et soit $U$ un voisinage ouvert et connexe de $p$. L'ensemble des points $q \in U$ tels que $\omega$ est holomorphe et non-nulle en $q$ est dense dans $U$; pour un tel point $q$ on a $\operatorname{ker} \omega(q)=L_{q}$ et alors $d f(q)\left(L_{q}\right)=0$. Donc

$$
d f(p)\left(L_{p}\right)=0 .
$$

Soit $(\Sigma, \varphi)$ une courbe tangente à $L$. Désignons par $W$ l'ouvert de Zariski où $f$ est holomorphe. Alors

$$
\left.d(f \circ \varphi)\right|_{\varphi^{-1}(W)}=0 .
$$


Comme $\varphi^{-1}(X-W)$ est un sous-ensemble analytique de $\Sigma$, nous devons avoir ou bien $d(f \circ \varphi)=0$ ou bien $\varphi(\Sigma) \subset X-W$; dans le premier cas $f \circ \varphi$ est constante. L'assertion suit de manière évidente.

\section{A.5 La tangence de deux feuilletages}

Soient $\mathcal{F}$ et $\mathcal{F}^{\prime}$ deux feuilletages (analytiques) à singularités isolées sur une surface analytique régulière et connexe $X$. Dans ce paragraphe on supposera que $\mathcal{F} \neq \mathcal{F}^{\prime}$.

Désignons par $L$ et $L^{\prime}$ les champs de droites tangentes aux feuilles de $\mathcal{F}$ et $\mathcal{F}^{\prime}$ respectivement. D'après A.2.3, on peut recouvrir $X$ par une famille $\left\{\left(U_{j},\left(x_{j}, y_{j}\right)\right)\right\}_{j \in J}$ de coordonnées locales connexes telles que, pour tout indice $j \in J$, il existe des équations locales

$$
\omega_{j}=0, \omega_{j}^{\prime}=0
$$

$\operatorname{sur} U_{j}$ de $\mathcal{F}$ et $\mathcal{F}^{\prime}$ respectivement. Alors

$$
\omega_{j} \wedge \omega_{j}^{\prime}=g_{j} d x_{j} \wedge d y_{j}, j \in J,
$$

où $g_{j}$ est une fonction holomorphe sur $U_{j}$ et $g_{j} \neq 0$ (voir A.2.6).

On déduit de A.2.3 que $\left\{\left(U_{j}, g_{j}\right)\right\}_{j \in J}$ définit un diviseur de Cartier effectif $D\left(\mathcal{F}, \mathcal{F}^{\prime}\right)$ de $X$ qui ne dépend pas des choix réalisés.

Définition A.5.1. Le diviseur $D\left(\mathcal{F}, \mathcal{F}^{\prime}\right)$ est le diviseur de tangence entre $\mathcal{F}$ et $\mathcal{F}^{\prime}$ (voir [5]).

Proposition A.5.2. On a les assertions suivantes :

a) Un point $p \in X$ n'appartient pas au support de $D\left(\mathcal{F}, \mathcal{F}^{\prime}\right)$ si et seulement si p n'est un point singulier ni de $\mathcal{F}$ ni de $\mathcal{F}^{\prime}$ et les droites tangentes, en $p$, aux feuilles de $\mathcal{F}$ et $\mathcal{F}^{\prime}$ par $p$ sont différentes.

b) Si $C$ est une séparatrice de $\mathcal{F}$ contenue dans le support de $D\left(\mathcal{F}, \mathcal{F}^{\prime}\right)$, alors $C$ est une séparatrice de $\mathcal{F}^{\prime}$.

Démonstration. a) Il suit immédiatement des définitions.

b) Il existe $p \in C$ qui n'est un point singulier ni de $C$ ni de $\mathcal{F}$ ni de $\mathcal{F}^{\prime}$. D'après l'hypothèse et A.1.3, la droite tangente $T_{q}(C)$ est tangente à la feuille de $\mathcal{F}$ par $q$, pour tout $q \in C$ au voisinage de $p$. Par a), il en est de même pour $\mathcal{F}^{\prime}$. On conclut par A.4.3.

\section{A.6 Feuilletages sur les fibrations}

Soit $h: X \rightarrow B$ une application analytique où $X$ est une surface analytique régulière connexe et $B$ une surface de Riemann. On suppose que $h$ 
est propre et qu'elle est une fibration localement triviale au sens $C^{\infty}$ (voir $[2])$.

On se donne un feuilletage à singularités isolées $\mathcal{F}$ de $X$.

Définition A.6.1. On dit que $\mathcal{F}$ est transverse aux fibres de $h$ si $\mathcal{F}$ est régulier et, pour chaque $p \in X$, la droite tangente en $p$ à la feuille de $\mathcal{F}$ par $p$ n'est pas tangente à la fibre de $h$ qui contient $p$.

Observation A.6.2. Prenons pour $\mathcal{F}^{\prime}$ le feuilletage défini par $h$ (exemples A.1.6 et A.2.4). Alors $\mathcal{F}$ est transverse aux fibres de $h$ si et seulement si $D\left(\mathcal{F}, \mathcal{F}^{\prime}\right)=0$.

Pour $\rho>0$ on note $D_{\rho}$ le disque de centre 0 et rayon $\rho$.

Théorème A.6.3. Supposons que $\mathcal{F}$ soit transverse aux fibres de $h$. Alors $h$ est une fibration localement analytiquement triviale. Plus précisément, pour chaque $b \in B$, il existe un voisinage $V$ de $b$, une variété analytique complexe, compacte, de dimension $1, X_{b}$ et un difféomorphisme analytique $\varphi: V \times X_{b} \rightarrow h^{-1}(V)$ tel que le diagramme suivant

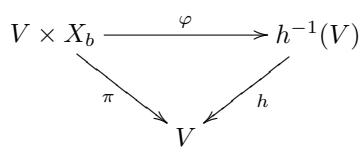

commute, où $\pi$ désigne la projection canonique, et de sorte que pour chaque $p \in X_{b}$, l'image de $V \times\{p\}$ par $\varphi$ est contenue dans une feuille de $\mathcal{F}$.

Démonstration. Notons $L$ le champ de droites tangentes aux feuilles de $\mathcal{F}$. Soit $b \in B$.

Soit $z: W \rightarrow D_{1}$ une coordonnée locale de $B$ avec $W$ un voisinage ouvert de $b$ telle que $z(b)=0$. Comme l'application

$$
\left.d h(p)\right|_{L_{p}}: L_{p} \longrightarrow T_{h(p)}(B)
$$

est un isomorphisme pour tout $p \in X$, le champ de vecteurs $\partial / \partial z$ se relève à un champ de vecteurs $\xi$ sur $U:=h^{-1}(W)$ tel que

$$
\xi_{p} \in L_{p}, d h(p)\left(\xi_{p}\right)=\frac{\partial}{\partial z}(h(p)), \forall p \in U .
$$

De la définition A.1.1 il suit que $\xi$ est analytique.

Le théorème d'existence, d'unicité et de dépendance analytique des conditions initiales pour les équations analytiques (voir [18]) et la compacité de $X_{b}$ entraînent l'existence de $\epsilon$, avec $0<\epsilon<1$, et d'une application analytique

$$
\psi: D_{\epsilon} \times X_{b} \longrightarrow U
$$


telle que, pour chaque $p \in X_{b}$, l'application $t \mapsto \psi(t, p)$ est la trajectoire de $\xi$ telle que $\psi(0, p)=p$. Du fait que $\xi$ relève $\partial / \partial z$ suit que le diagramme

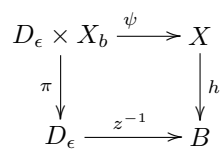

est commutatif, où $\pi$ désigne la projection canonique.

Par ailleurs, la restriction de $\psi$ à $D_{\epsilon} \times\{p\}$ est une courbe tangente à $L$ pour tout $p \in X_{b}$. Alors $\psi\left(D_{\epsilon} \times\{p\}\right)$ est contenu dans une feuille de $\mathcal{F}$ pour tout $p \in X_{b}$. Comme $\psi(0, p)=p$ et $d \psi(0, p)\left(T_{0}\left(D_{\epsilon}\right)\right)=L_{p}$, on voit que $d \psi(0, p)$ est bijective pour tout $p \in X_{b}$. Alors $\psi$ est un isomorphisme local au voisinage de $(0, p)$. Quitte à considérer $\epsilon$ suffisamment petit, de cela et de la compacité de $X_{b}$, on déduit que $\psi$ est injective. Puisque toutes les fibres de $h$ sont difféomorphes, il vient

$$
\psi\left(D_{\epsilon} \times\{p\}\right)=h^{-1}(V),
$$

où $V:=z^{-1}\left(D_{\epsilon}\right)$.

On définit $\varphi: V \times X_{b} \rightarrow U$ par

$$
\varphi(v, p)=\psi\left(z^{-1}(v), p\right), v \in V, p \in X_{b},
$$

ce qui complète la preuve.

Corollaire A.6.4. Supposons que $\mathcal{F}$ soit transverse aux feuilles de $h$; notons $F$ une feuille arbitraire de $\mathcal{F}$. Alors, l'application

$$
\left.h\right|_{F}: F \longrightarrow B
$$

est un revêtement non-ramifié pour la topologie fine de $F$.

Démonstration. Soit $b \in B$. Soient $X_{b}, V$ et $\varphi$ comme dans A.6.3. Alors, d'après ce résultat, l'ensemble $F \cap h^{-1}(V)$ est homéomorphe, pour la topologie fine de $F$, à $V \times S$ où $S \subset X_{b}$ et sur $S$ on a pris la topologie discrète. De plus, cet homéomorphisme commute avec $h$ et la projection canonique $V \times S \rightarrow V$.

Corollaire A.6.5. Supposons que $\mathcal{F}$ soit transverse aux fibres de $h$. Fixons $b \in B$ et choisissons $x \in h^{-1}(b)$; notons $F$ la feuille passant par $x$. Alors, tout chemin continu $\gamma:[0,1] \rightarrow B$ avec $\gamma(0)=b$ admet un relèvement continu unique $\gamma^{\prime}:[0,1] \rightarrow X$ tel que

$$
h \circ \gamma^{\prime}=\gamma, \gamma^{\prime}(0)=x \text { et } \gamma^{\prime}([0,1]) \subset F .
$$

De plus, $\gamma(1)$ ne dépend que de la classe d'homotopie à extrémités fixes de $\gamma$. 
Démonstration. Il découle de A.6.4 et A.1.16.

Le corollaire A.6.5 nous dit, en particulier, que si $\gamma$ est fermé, alors il induit une application $x \mapsto \gamma^{\prime}(1)$ de $X_{b}:=h^{-1}(b)$ dans lui-même et que ceci définit un homomorphisme de $\pi_{1}(B, b)$ dans le groupe des transformations bijectives $X_{b} \rightarrow X_{b}$ que l'on appelle l'holonomie.

Proposition A.6.6. L'holonomie est un homomorphisme de $\pi_{1}(B, b)$ dans le groupe $A u t\left(X_{b}\right)$ des automorphismes analytiques de $X_{b}$. Si cet homomorphisme est trivial, alors il existe un isomorphisme analytique $\varphi: X \rightarrow$ $B \times X_{b}$ tel que :

a) le diagramme

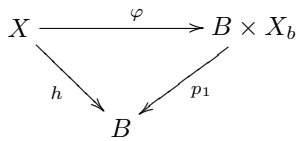

commute;

b) les feuilles de $\mathcal{F}$ sont de la forme

$$
\varphi^{-1}(B \times\{x\}), x \in X_{b} .
$$

Démonstration. On définit

$$
\varphi(z):=\left(h(z), x_{z}\right), z \in X
$$

où $x_{z} \in X_{b}$ est dans la même feuille que $z$. La preuve suit de A.6.3. 


\section{Bibliographie}

[1] L. Ahlfors, Complex Analysis, 3rd. ed. McGraw-Hill, New York, 1979.

[2] W. Barth, C. Peters, A. Van de Ven, Compact Complex Surfaces, Springer Verlag, 1984.

[3] A. Beauville, Complex Algebraic Surfaces, Cambridge Univ. Press, 1996.

[4] E. Brieskorn, Plane Algebraic Curves, Birkhï $\frac{1}{2}$ ser, 1986.

[5] M. Brunella, Feuilletages holomorphes sur les surfaces complexes compactes, Ann. Sci. Écol. Norm. Sup., $4^{a}$ sér., t. 30 (1997), p. 569-594.

[6] M. Brunella, Birational Geometry of Foliation, First latin Americam Congress of Mathematicians, Impa, 2000 :

http ://www.impa.br/Publicacoes/Monografias/index.html.

[7] C. Camacho, A. Lins Neto, Teoria Geométrica das Folheações, Projeto Euclides, IMPA, Rio de janeiro, 1977.

[8] C. Camacho, Quadratic Forms and Holomorphic Foliations on Singular Surfaces, Math. Annalen 282 (1988), pags. p. 177-184.

[9] C. Camacho, P. Sad, Invariant Varieties Through Singularities of Holomorphic Vector Fields, Ann. of Math. 115, (1982) p. 579-595.

[10] D. Cerveau, Densité des feuilles de certaines équations de Pfaff à deux variables, Ann. Inst. Fourier, 33,1 (1983), p. 185-194.

[11] A. E. Eremenko, Meromorphic solutions of algebraic differential equations, Russian Math. Surveys 37 :4 (1982), p.61-95.

[12] G. Fischer, H. Grauert, Lokaltriviale Familien kompakter complexer Mannifaltigkeiten, Nachr. Akad. Wiss. Göttingen II (1965), p. 89-94.

[13] M. Fuchs, Ueber Differentialgleichungen deren Integrale feste Verzwueigungspunkte besitzen, Math. Werke 2, p. 355.

[14] P. Griffiths, J. Harris, Principles of Algebraic Geometry, John Wiley \& Sons, 1978. 
[15] R.C Gunning, Introduction to Holomorphic Functions of Several Variables, Wadsworth and Brooks/Cole, 1990.

[16] J. Harris, Algebraic Geometry, Springer-Verlag, 1992.

[17] R. Hartshorne, Algebraic Geometry, Springer, 1977.

[18] E. Hille, Ordinary Differential Equations in the Complex Domain, John Wiley and Sons, 1976.

[19] H. Hironaka, Resolution of singularities of an algebraic variety over a fields of characteristic zero I, II, Ann. Math. 79 (1964), p. 109-326.

[20] E.L. Ince, Ordinary differential equations, Dover, 1926.

[21] J.P. Jouanolou, Hypersurfaces solution d'une équation de Pfaff analytique, Math. Annalen. 232 (1978), p. 239-245.

[22] S. Kobayashi, Hyperbolic Manifolds and Holomorphic Mappings, Marcel Dekker, 1970.

[23] S. Lang, Algebra, Addison-Wesley Publishing Company, Inc. 1965.

[24] J. Malmquist, Sur les fonctions à un nombre fini des branches définies par les équations différentielles du premier ordre, Acta Math., 36 (1913), p. 297-343.

[25] J. Malmquist, Sur les fonctions à un nombre fini de branches satisfaisant à une équation différentielle du premier ordre, Acta Math. 42 (1920), p. 59-79.

[26] J. Malmquist, Sur les fonctions à un nombre fini de branches satisfaisant à une équation différentielle du premier ordre, Acta Math. 74 (1941), p. 175-196. MR 7-298.

[27] M. Matsuda, First-order algebraic differential equations. A differential algebraic approch, Lectures Notes in Math. 804, Springer 1980.

[28] I. Pan, M. Sebastiani, Sur les équations différentielles algébriques admettant des solutions avec une singularité essentielle, Ann. de l'Inst. Fourier, Tome 51, 6 (2001) p. 1621-1633 .

[29] H. Poincaré, Sur un théorème de M. Fuchs, C.R. Acad. Sci. Paris 99 (1984), p.75-77 et Acta Math. 7, (1985) p.1-32.

[30] B. Scárdua, Holomorphic Foliations Transverse to Fibrations on Hyperbolic Manifolds, Complex Variables : Applications and Theory, Vol. 46 (2001), p. 219-240.

[31] I. R. Shafarevich, Basic Algebraic Geometry 1, Second Edition, Springer-Verlag, 1994. 
[32] C. L. Siegel, Topics in Complex Function Theory : vol. I, John Wiley and Sons, 1969.

[33] H. Wittich, Zur Theorie der Riccatischen Differentialgleichung, Math. Ann., 127 (1954), p. 433-450.

[34] C. C. Yang, A note on Malmquist's theorem on first-order differential equations, Yoko. Math. J., 20 (1972), p. 115-125.

[35] K. Yosida, A generalisation of a Malmquist's theorem, Japan. J. Math., 9 (1933), p. 253-256.

[36] O. Zariski, P. Samuel, Commutative Algebra, Volumes I and II, Springer Verlag, 1960.

Ivan Pan

Instituto de Matemática-UFRGS

Av. Bento Gonçalves, 9500

91501-970 Porto Alegre-RS

e-mail : pan@mat.ufrgs.br
Marcos Sebastiani

Instituto de Matemática-UFRGS

Av. Bento Gonçalves, 9500

91501-970 Porto Alegre-RS 